\title{
Generation and Maintenance of Recirculations by Gulf Stream Instabilities
}

\author{
by
}

Natalia Yurievna Beliakova

M.Sc. in Applied Mathematics, Moscow State University, Moscow, Russia

(1989)

Submitted in partial fulfillment of the

requirements for the degree of

Doctor of Philosophy

at the
MASSACHUSETTS INSTITUTE OF TECHNOLOGY
and the

WOODS HOLE OCEANOGRAPHIC INSTITUTION

February 1999

(C) Natalia Y. Beliakova 1998

The author hereby grants to MIT and to WHOI permission to reproduce and to distribute publicly paper and electronic copies of this thesis document in whole or in part, and to grant others the right to do so.

Signature of Author.......

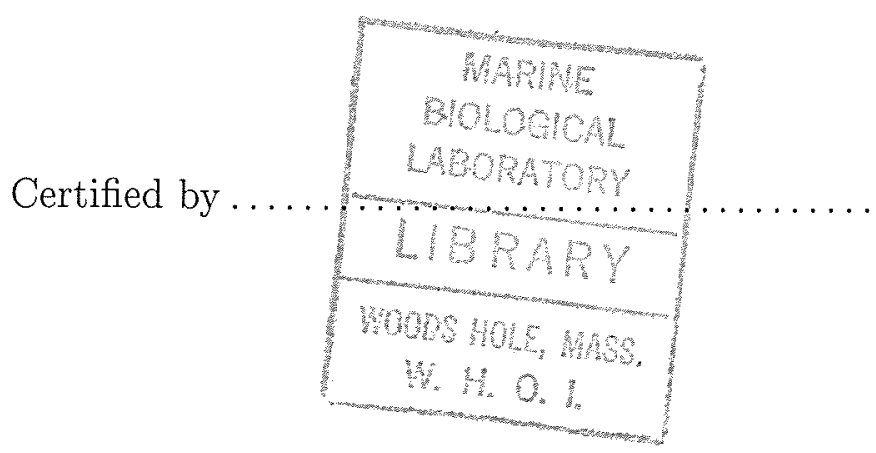

Accepted by
Joint Program in Physical Oceanography Massachusetts Institute of Technology Woods Hole Oceanographic Institution

September 22, 1998 $\therefore 1 \ldots \ldots . . . .$.

Glenn R. Flierl Professor Massachusetts Institute of Technology Thesis Superyisor

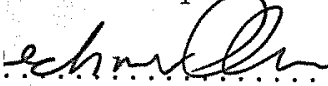

W. Brechner Owens Chairman, Joint Committee for Physical Oceanography Massachusetts Institute of Technology Woods Hole Oceanographic Institution 
In Loving Memory of My Husband, Gera Panteleyev 


\title{
Generation and Maintenance of Recirculations by Gulf Stream Instabilities
}

\author{
by \\ Natalia Yurievna Beliakova \\ Submitted to the Joint Program in Physical Oceanography \\ on September 22, 1998, in partial fulfillment of the \\ requirements for the degree of \\ Doctor of Philosophy
}

\begin{abstract}
This thesis studies the problems of generation and maintenance of recirculations by Gulf Stream instabilities. Observations show that the horizontal structure of the jet and its recirculations suffer significant changes in time. Here, the role of internal dynamics of the jet is isolated as one of the possible sources of such variability, and the differences between barotropic and baroclinic instabilities are investigated.

The problem of recirculation development is considered in a framework of a free spin down of the 2-layer and the $1 \frac{1}{2}$-layer, zonally symmetric, quasi-geostrophic jets. Linear stability analysis shows that in strongly baroclinic basic flows, eddies are capable of driving recirculations in the lower layer through the residual meridional circulation. In strongly barotropic jets, the linearly most unstable wave simply diffuses the jet. Nonlinear stability analysis indicates that recirculations are robust features of the 2-layer model. The strength of recirculations is a function of the model's parameters. It increases with a decrease in the value of the nondimensional $\beta$ due to potential vorticity homogenization constrained by enstrophy conservation. The recirculation strength is a non-monotonic function of the baroclinic velocity parameter; it is the strongest for strongly baroclinic basic flows, weakest for flows with intermediate baroclinic structure and of medium strength for strongly barotropic basic flows. Such non-monotonic behavior is the result of two different processes responsible for the recirculation development: linear eddy-mean How interactions for strongly baroclinic basic flows and strongly nonlinear eddy-eddy and eddy-mean flow interaction for strongly barotropic flows. In the case of the reduced-gravity model, recirculations develop only for infinite deformation raduis. Basic flows with finite deformation radius are only weakly supercritical and therefore produced negligible recirculations after equilibration.

The problem of maintenance of the recirculations is coupled to the questions of existence of low frequency variability and of multiple dynamical regimes of a system
\end{abstract}


consisting of a quasi-geostrophic jet and its recirculations. The problem is studied in a framework of a 2-layer or a reduced-gravity colliding jets model which has no windforcing. Instead, it is forced by inflows and outflows through the open boundaries. Only the western boundary of the domain is closed, and a free slip boundary condition is used there. The results of the numerical experiments show that when only the mechanism of barotropic instability is present, the model has two energy states for a wide range of interfacial friction coefficients. The high energy state is characterized by well-developed recirculations and displays strong variability associated with either large recirculating gyres and a weak eddy field or small recirculations and a strong eddy field. The low energy state is characterized by large meridional excursions in the separation point and large amplitude, westward propagating meanders that produce strong rings after interacting with the western wall.

For physically relevant bottom friction values, the presence of baroclinic instability in the recirculation regions of the 2-layer model allows for a unique dynamical regime characterized by well-developed recirculations in both layers. The low-frequency variability associated with the regime is weak and is related to meridional shifts in the position of the jet, to wrapping of the recirculations around each other, and to pulsations in their zonal extent. For strong bottom friction, the 2-layer model has only the mechanism of barotropic instability which reduces it to a $1 \frac{1}{2}$--layer configuration; the model displays two dynamical regimes and strong low frequency variability in the upper layer, while the lower layer is strongly frictional.

Thesis Supervisor: Glenn R. Flierl

Title: Professor

Massachusetts Institute of Technology 


\section{Acknowledgments}

Graduate school at MIT and WHOI was one of the most interesting and intense experiences in my life. It started not just as a new academic environment, but also as a new country. Perhaps, two academic institutions cannot fully represent the whole U.S., but in terms of the number of ingenious people per square meter, MIT/WHOI is similar to a delta function. I am sure that many exciting events await for me in the future, but being part of MIT and WHOI offers something very unique: a group of people, from different cultures and of different generations, united by their interest in oceans and atmospheres (not just on this planet), people with amazing curiosity and drive.

I am indebted to many here who ignited my interest in subjects that I had never heard about before and who changed my way of thinking and not(!) thinking. First of all, I would like to thank my advisor, Glenn Flierl, for his immense patience and great support, both scientific and personal. Glenn's character combines ultimate kindness, balance, expertise and intellect (intimidating at times), which make him a

perfect advisor. I would also like to thank my committee members: Joe Pedlosky, Paola Rizzoli, Karl Helfrich and Nelson Hogg, for their tremendous help and numerous and important contributions to my research. I am grateful to Larry Pratt, who was my advisor during the first two years of graduate school, for exciting my deep interest in instability theory, nonlinear dynamics and chaos, - subjects that defined the framework of this thesis. I enjoyed interesting discussions of low-frequency variability in wind-driven models with Steve Meacham.

MIT/WHOI Joint Program offered a wonderful and stimulating company of fellow students. I especially would like to thank Francois Primeau for endless discussions of various scientific problems, Kirill Pankratov for useful advice on the numerical methods in fluid dynamics and Pavel Berloff for explanations of low frequency variability in wind-driven models. Michael Chechelnitsky and Xiaoyun Zang answered numerous questions on data analysis and statistics. 
MIT/WHOI community and working on this thesis helped tremendously after I lost my husband, Gera, in a horrifying accident. It was hard to continue living everyday life, but so many people offered comfort and support, and I am indebted to them for being with me and helping me to go through those difficult times. I would like to dedicate this work to Gera, who has always been an immense source of love, encouragement and happiness to me and who taught me so many wonderful things about this world.

Financial support for this research was provided by NSF grant number OCE9617848. Numerical calculations for Chapters 3, 4 and 5 were performed on Cray supercomputer facility at NCAR. Plots adapted from other research papers were drafted by Bud Brown.

Through MIT and WHOI, I found many wonderful friends: Chris Edwards, Emilie Hooft, Richard Wardle and Polina Golland. They expanded my horizons and fulfilled my life with great joy and excitement. Emilie and Chris introduced me to their families, and I found people who were kindred to me in spirit and who made me feel home in this new country. Richard helped to develop my parental instincts. Natasha Trinko-Berloff was a constant source of energy and support. Hugh's own life experience and his love were healing.

Finally, I would like to thank my parents, my two wonderful grandmothers and Tania for their love, care and giving spirit. Lida and Valentin, thank you for guiding me so many times, always successfully. Alla and Pavel, thank you for being my family. 


\section{Contents}

$\begin{array}{ll}\text { Abstract } & 3\end{array}$

Acknowledgments $\quad 5$

1 Introduction $\quad 11$

1.1 Background and Motivation ................. 11

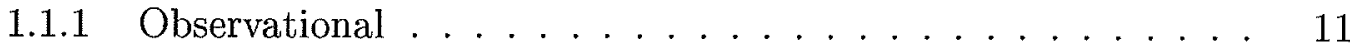

1.1 .2 Dynamical ........................... 16

1.2 Outline of the Thesis . . . . . . . . . . . . . 21

2 Generation of Recirculations in a Two-Layer Quasi-Geostrophic Zonally Symmetric Jet Model 24

2.1 Model Formulation . . . . . . . . . . . . . . . 25

2.1.1 Model Equations .................. 25

2.1.2 Dimensional Scales .................. 25

2.1.3 Nondimensional Parameters . . . . . . . . . . . 26

2.1.4 Sponge Operator ................... 26

2.1.5 Basic Flow ........................ 28

2.1 .6 Initialization . . . . . . . . . . . . . 28

2.2 Linear Stability Analysis of a Two-Layer Model . . . . . . . . . 28

2.2.1 Linear Model Formulation . . . . . . . . . . . . 30

2.2.2 Method of Solution ............... 30 
2.2.3 Maximum Growth Rates of Unstable Modes . . . . . . . . 32

2.2.4 Wave - Zonal Mean Flow Interactions . . . . . . . . . . 35

2.2.5 Eddy Energy Equation . . . . . . . . . . . . 38

2.2.6 Transformed Eulerian Mean Circulation .......... 40

2.2.7 Relevance to Fully Nonlinear Calculations . . . . . . . . . . 44

2.3 Nonlinear Analysis of a Two-Layer Model . . . . . . . . . . . 47

2.3.1 The Flow Evolution . . . . . . . . . . . . 47

2.3 .2 The Equilibrated Jet Structure . . . . . . . . . 50

2.3.3 Potential Vorticity Mixing . . . . . . . . . . . . 52

2.3.4 Relative Strength of the Recirculations . . . . . . . . . 70

2.4 Results for the Reduced-Gravity Model . . . . . . . . . . . 77

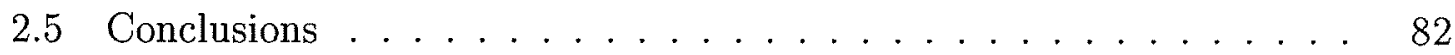

3 Colliding Jets Model Formulation $\quad 85$

3.1 Introduction . . . . . . . . . . . . . . 85

3.2 Model Formulation . . . . . . . . . . . . . 86

3.2.1 Governing Equations . . . . . . . . . . . . 86

3.2 .2 Dimensional Scales ... . . . . . . . . . . . 87

3.2.3 Model Domain ................ 87

3.2.4 Nondimensional Parameters . . . . . . . . . . . 87

3.2.5 Forcing and Boundary Conditions . . . . . . . . 88

3.2 .6 Sponge Operator .................... 90

3.2 .7 Numerical scheme . . . . . . . . . . . . . . . . 93

3.2.8 The Inversion of the Potential Vorticity Field . . . . . . . 93

3.2 .9 Initialization of the Model . . . . . . . . . . . . . 94

3.3 Energetics of the Model . . . . . . . . . . . . . 97

3.3.1 Energy Equation . . . . . . . . . . . . . . . 97

3.3.2 Energy Sources and Sinks . . . . . . . . . . 98 
3.3.3 Energy Convergence Between the Time Mean Flow and Perturbations ........................ 100

3.4 Ring Dynamics at the Western Wall . . . . . . . . . . . . 102

4 Results for Reduced-Gravity Colliding Jets Model 105

4.1 Model Formulation . . . . . . . . . . . . . . . 105

4.1 .1 Governing Equation ................ 105

4.1 .2 Energetics . . . . . . . . . . . . . 106

4.2 Linear Stability Analysis of Steady Antisymmetric Solutions . . . . 108

4.2.1 Steady States vs Interfacial Friction Coefficient . . . . . . . 108

4.2.2 Growth Rates, Phase Speeds and Structures

of Unstable Modes . . . . . . . . . . . . . . 111

4.3 Sensitivity Study for Various Interfacial Friction Coefficients . . . . . 115

4.3 .1 Classification of States . . . . . . . . . . 115

4.3 .2 Examples of States ... . . . . . . . . . 120

4.4 Reference Run: State $E \ldots \ldots$. . . . . . . . . . 133

4.4.1 Dimensional Parameters ............... 133

4.4.2 Energy Time Series and Their Spectra . . . . . . . . . . 134

4.4 .3 Energy Histograms . . . . . . . . . . . . . . . 139

4.4 .4 Time Mean Flow . . . . . . . . . . . . . . . . 142

4.4.5 Time Mean Eddy Variability . . . . . . . . . . . . . 145

4.4.6 Statistical Differences in the Eddy and Time Mean Flow Energy 145

4.4 .7 Enstrophy . . . . . . . . . . . . . . 147

4.4.8 Mean Fields Associated with Different Energy States . . . . 148

4.4 .9 Typical Energy Cycle . . . . . . . . . . . . 153

4.4.10 Mass and Potential Vorticity Balances . . . . . . . . . 162

4.4.11 Transitions Between High and Low Energy States . . . . . . 166

4.5 Discussion of Nonsymmetric Inflow Conditions . . . . . . . . . 170

4.6 Summary and Discussion . . . . . . . . . . . . . . 173 
5.1 Introduction . . . . . . . . . . . . . 177

5.2 Comments on Strong Bottom Friction . . . . . . . . . . . . 178

5.3 Sensitivity Study vs Bottom Friction Coefficient . . . . . . . . . 179

5.4 Reference Run . . . . . . . . . . . . . . . . . 183

5.4 .1 Dimensional Parameters ................. 183

5.4 .2 Time Mean Flow . . . . . . . . . . . . . . . 188

5.4 .3 Energetics . . . . . . . . . . . . . . 190

5.4 .4 Time Mean Eddy Fields . . . . . . . . . . . 195

5.4.5 EOF Analysis of Low Frequency Variability . . . . . . . . 197

5.4.6 EOF Analysis of High Frequency Variability . . . . . . . 200

5.5 The Role of Baroclinic Instability in Low Frequency Variability . . . 201

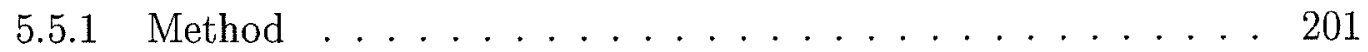

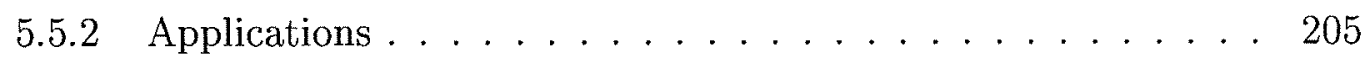

5.6 Summary and Discussion . . . . . . . . . . . . 206

6 Conclusions $\quad 209$

$\begin{array}{ll}\text { Bibliography } & 220\end{array}$ 


\section{Chapter 1}

\section{Introduction}

\subsection{Background and Motivation}

\subsubsection{Observational}

The discoveries of the intense recirculating gyres south and north of the Gulf Stream explained why the transport of the jet downstream of Cape Hatteras $(150 \mathrm{~Sv})$ is five times larger than the maximum wind-driven transport near the Straits of Florida (30 $\mathrm{Sv})$. The anticyclonic recirculation west of $50^{\circ} \mathrm{W}$ with the transport of about $60 \mathrm{~Sv}$ was first proposed by Worthington [40]. By incorporating both mass and geostrophy balance into an inverse model applied to the same data set, Wunsch [41] and Wunsch and Grant [42] postulated a cyclonic recirculation west of $50^{\circ} \mathrm{W}$ and north of $30^{\circ} \mathrm{N}$ with the transport of $20 \mathrm{~Sv}$.

By using long-term moored instruments near $55^{\circ} \mathrm{W}$, Schmitz [37] described a flow regime that consisted of narrow (200 km wide) jet-like currents with eastward velocities on the flanks of the Gulf Stream and westward velocities further north and south. These currents were termed as "weakly depth-dependent" meaning that velocity amplitudes were similar at thermocline and abyssal depths and varied between 


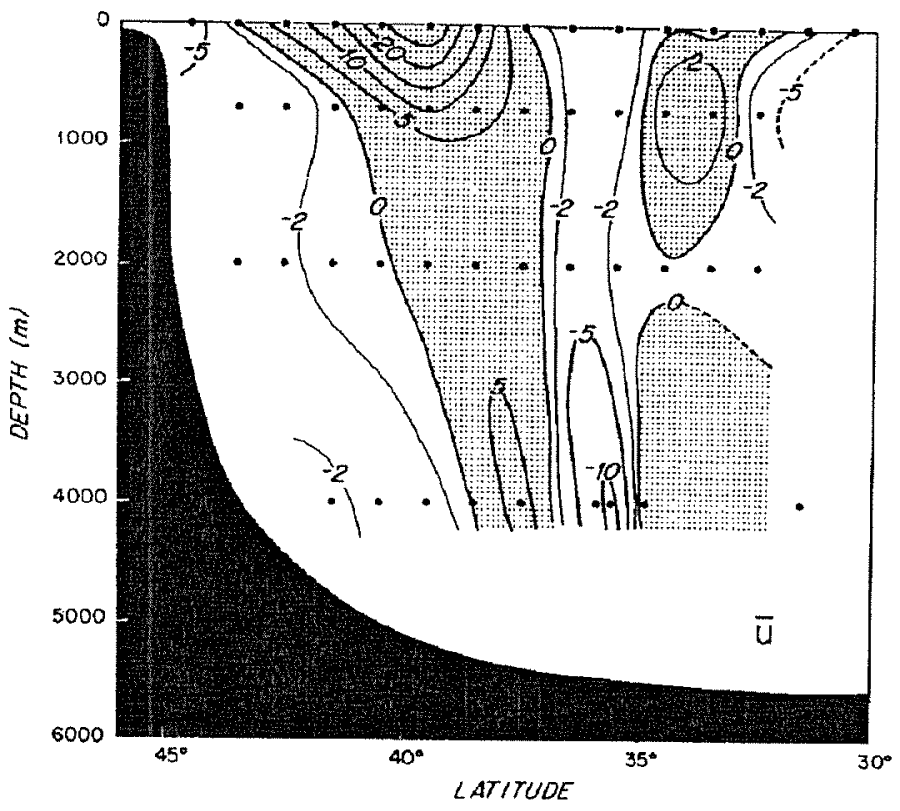

Figure 1-1: Contours of zonal velocity $\left(\mathrm{cm} \mathrm{s}^{-1}\right)$ along $55^{\circ} \mathrm{W}$. Adapted from Richard$\operatorname{son}[32]$.

6 and $10 \mathrm{~cm} \mathrm{~s}^{-1}$. Schmitz noted that these currents might be partly responsible for enhancing the transport of the Gulf Stream.

The combination of surface drifters, SOFAR floats and current meters allowed Richardson [32] to further study the vertical and horizontal structure of the Gulf Stream near $55^{\circ} \mathrm{W}$. In a region of high eddy kinetic energy and its gradient, he found an eastward jet flanked by two recirculations. Estimated at $93 \mathrm{~Sv}$, the total mean volume transport of the Gulf Stream was three times larger than that near the Straits of Florida. Such an enhancement was due to additional $41 \mathrm{~Sv}$ and 29 Sv carried respectively by the $300 \mathrm{~km}$ wide northern and southern counter-currents. Their westward velocities did not change significantly with depth and ranged from 2 to $10 \mathrm{~cm} \mathrm{~s}^{-1}$ (Figure 1-1).

The northern recirculation was examined by Hogg et al. [14]. Based on longterm current and tracer measurements, they concluded that the Northern Recirculating Gyre, located between the New England Seamount Chain and the Grand Banks, 


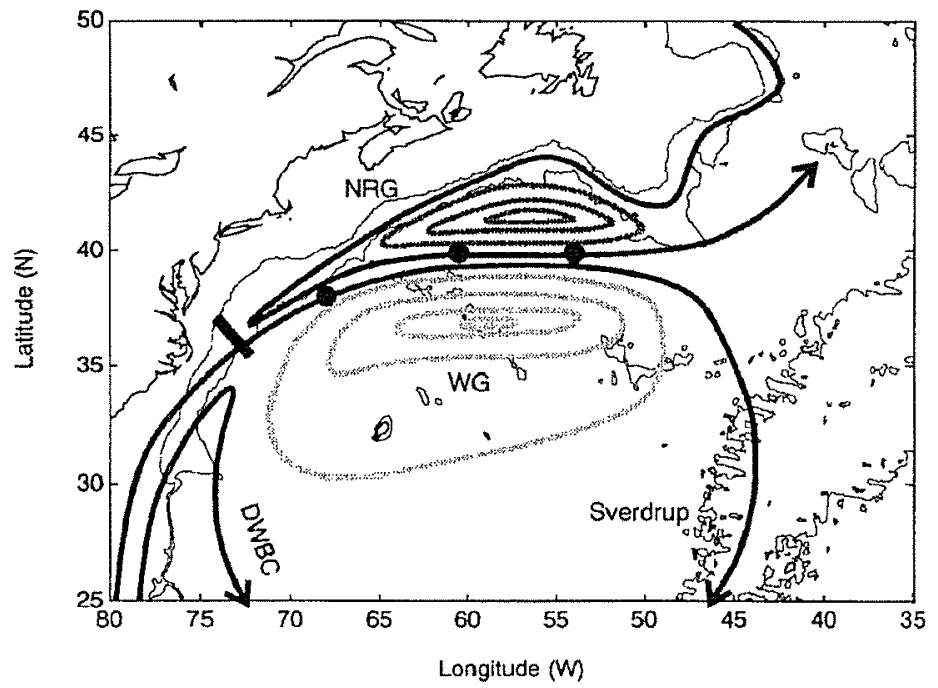

Figure 1-2: Schematic circulation in the western North Atlantic. Adapted from Hogg $[12]$.

transported about $40 \mathrm{~Sv}$ of water to the west of $63^{\circ} \mathrm{W}$. Half of this water was returned back to the deep Gulf Stream. Consistent with the previous studies, the vertical structure of the flow between $500 \mathrm{~m}$ and the bottom was weakly depth dependent and similar to that of the southern recirculation. It was found also that the northern recirculation exchanged water properties with the Deep Western Boundary Current. Such tracers as freon and oxygen were further advected into the interior and homogenized within the gyre.

Estimates of the synoptic transport of the Gulf Stream were obtained by Hogg [12]. Synoptic mean circulation is different from traditional Eulerian mean circulation in that it is computed in a coordinate frame aligned with the instantaneous axis of the Stream, and therefore it accounts for changes both in the path and in the meandering activity of the jet. Hogg showed that downstream of Cape Hatteras, the synoptic transport was depth-independent and reached $150 \mathrm{~Sv}$ between $55^{\circ} \mathrm{W}$ and $60^{\circ} \mathrm{W}$. He argued that about $120 \mathrm{~Sv}$ might be evenly partitioned between the northern and southern recirculations in the synoptic mean frame compared to about $60 \mathrm{~Sv}$ in Eulerian mean frame. Figure 1-2 shows a schematic circulation in the western 

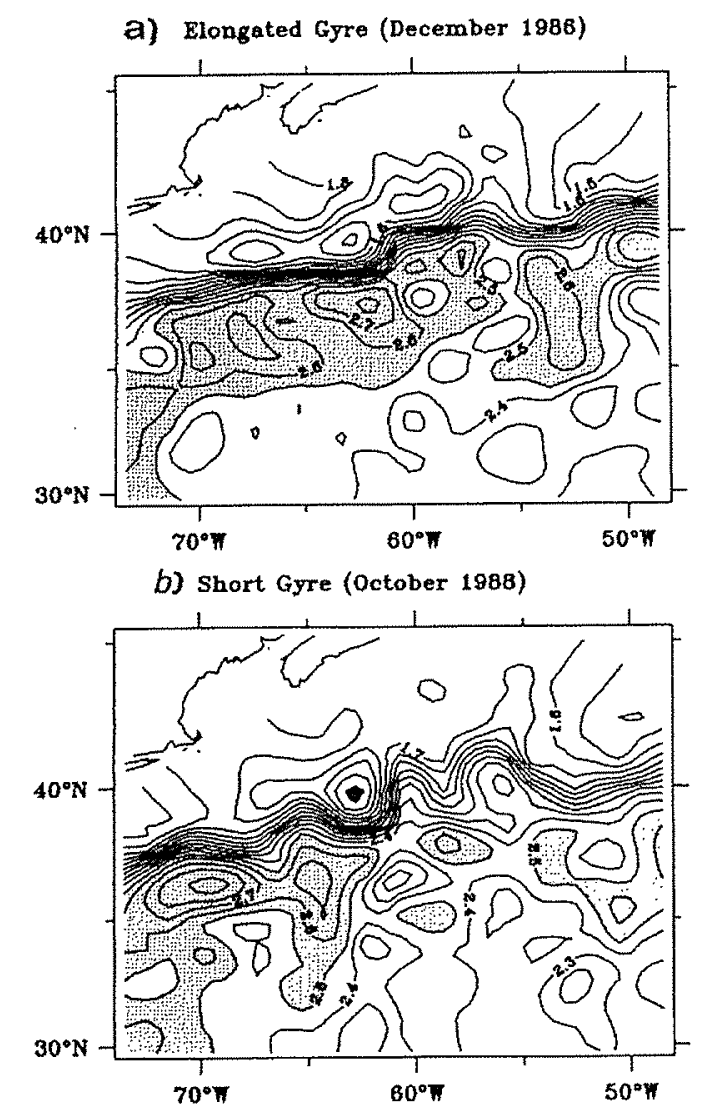

Figure 1-3: Maps of monthly averaged sea surface height anomalies in western North Atlantic for December 1986 (a) and October 1988 (b). Shaded area represents anomalies larger than $2.5 \mathrm{~m}$ in the southern recirculating gyre. Adapted from Kelly et al. [19].

North Atlantic which is characterized by the eastward flowing Gulf Stream and two recirculations.

Observations revealed that the horizontal structure of the Gulf Stream recirculations is strongly time-dependent. For example, the Geosat altimeter observations of sea surface height in the Gulf Stream (Kelly et al. [19]) have demonstrated a trend over a period of two years from a state with elongated recirculating gyres and a weakly meandering jet to a state with contracted gyres and a strongly meandering eastward jet (Figure 1-3). There was no significant correlation between the gyre fluctuations and the non-seasonal wind curl line tilt. However the surface transport 
fluctuations, which Kelly et al. suggested were due to variations in the longitudinal size of the recirculations, were correlated with the changes in both the jet's path and in the local wind stress fields. The dominant mode of variability had a time scale of 5 to 9 months.

Observational data of sea surface temperature of the Gulf Stream between $75^{\circ} \mathrm{W}$ and $60^{\circ} \mathrm{W}$ from April of 1982 to December of 1989 revealed a dominant 9month period in the meandering intensity of the jet (Lee and Cornillon [20]). The correlation between the meandering intensity of the jet and the temporal variation in the mean position of the jet, which had annual and inter-annual oscillations, was small. Lee and Cornillon suggested that the 9-month cycle might be connected to the instability time scale.

The recirculations might also affect the dynamics of the Gulf Stream by advecting a given meander westward and thus by stimulating the ring formation process. On the other hand, due to this westward advection, rings ultimately rejoin the Gulf Stream and return some of the heat and potential vorticity to the jet (Richardson [31]). Warm core rings which are formed on the northern side of the Stream bring anticyclonic vorticity into the cyclonic Northern Recirculating gyre. The opposite is true for the cold core rings introducing cyclonic vorticity into the anticyclonic southern recirculating gyre. Therefore, the existence of the rings may generally tend to decelerate the flow in the rectified regions. An increase in the strength and amplitude of the meanders creates a higher probability of having rings formed and, therefore, will enhance the mean transfer of heat and momentum between the sub-tropical and the sub-polar gyres.

Low-frequency variability is well documented in observations of the world oceans. Twenty two years of hydrographic data along $137^{\circ}$ show strong inter-annual bimodal path variations in the Kuroshio (Qiu and Joyce [30]). The net transport of the Kuroshio system which includes the jet and its recirculations increases by $30 \%$ during the meander-path years. The increase is primarily due to the decrease in the 

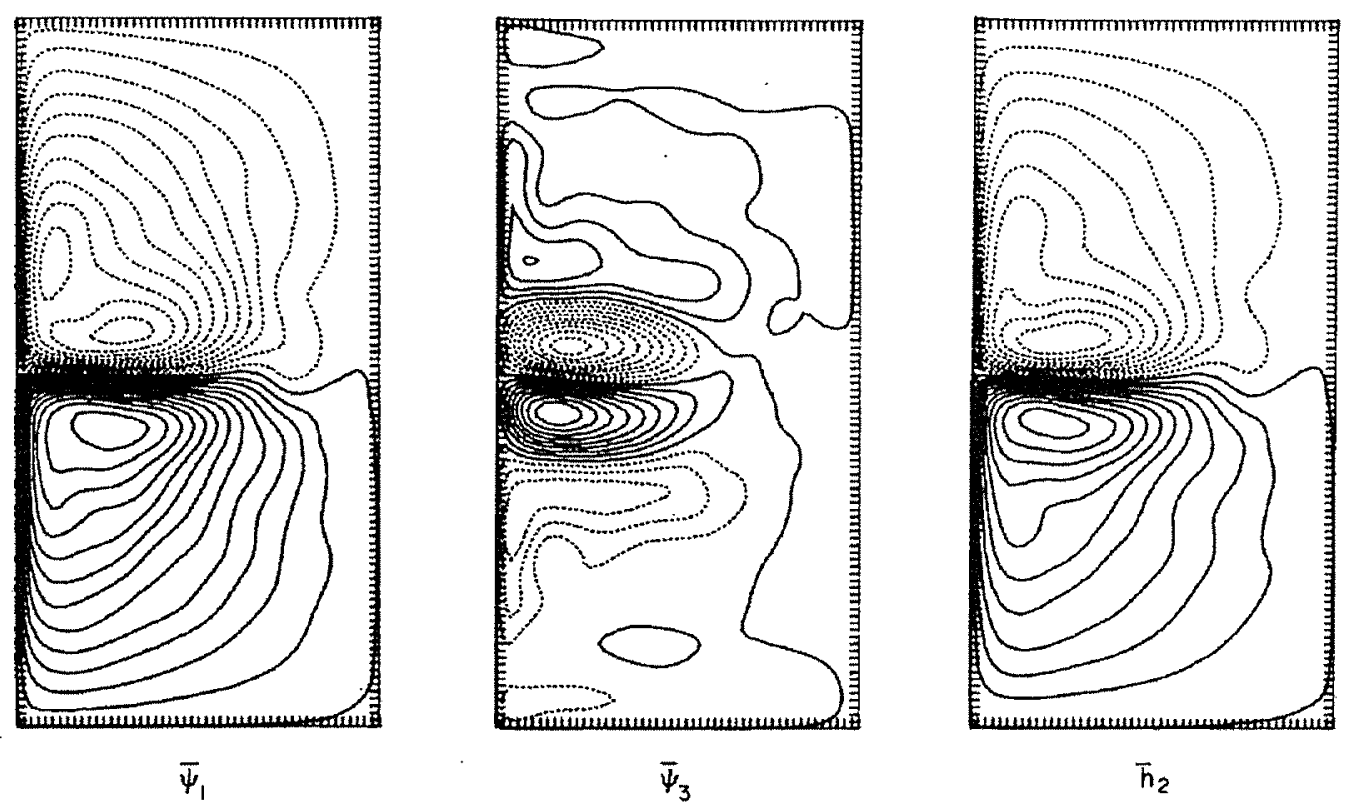

Figure 1-4: Time averaged fields of upper layer (left) and lower layer (middle) streamfunction and of interface height (right). Adapted from Holland and Rhines [16].

recirculation strength. Thus, recirculations are strongest during the straight-path years. Observations of East Australian current (Roemmich and Cornuelle [33]) and of Brazil and Malvinas currents (Olson et al. [26]) also display strong inter-annual variability.

\subsubsection{Dynamical}

Strong westward recirculations appeared naturally in eddy-resolving, wind-driven, general circulation models (for a review see Holland et al. [17]). The results of these numerical experiments showed the importance of eddies in the local potential vorticity balance of the recirculations. For example, in a two-layer quasi-geostrophic wind-driven model of Holland and Rhines [16], two tight, intense, inertial recirculations developed on the flanks of an eastward jet in both layers (Figure 1-4). The circulation theorem applied to the time mean streamlines implied that the abyssal recirculations were forced from above by eddy thickness (or eddy heat) fluxes, and 

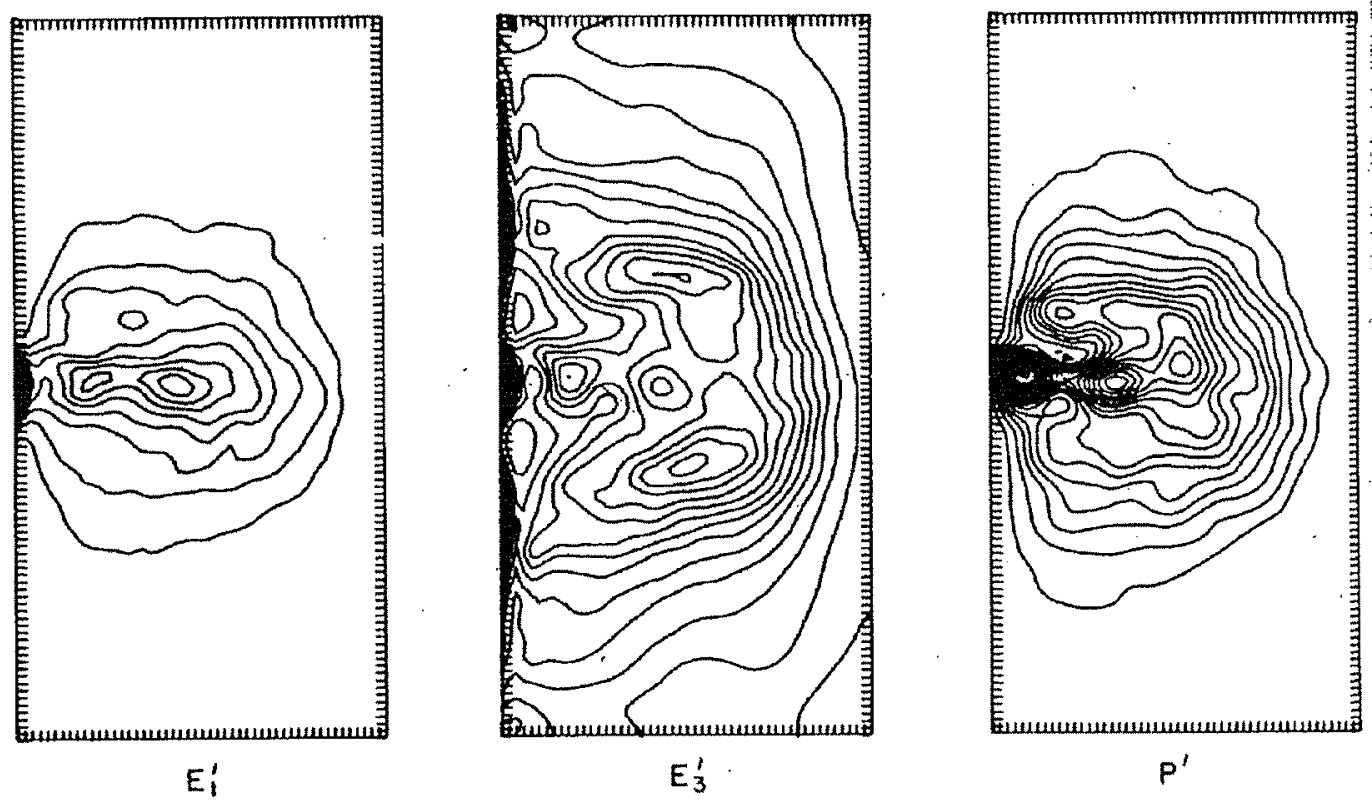

Figure 1-5: Time averaged upper layer (left) and lower layer (middle) eddy kinetic energy and eddy potential energy (right). Adapted from Holland and Rhines [16].

the upper layer recirculations were driven by the eddy Reynolds stresses (or eddy momentum fluxes). Energy transformation between the eddies and the mean flow was maximum in the areas of maximum eddy kinetic and eddy potential energy. The latter maxima occurred in a region of barotropically and baroclinically unstable eastward jet and inertial recirculations (Figure 1-5).

These results compared well with observed surface eddy kinetic (Figure 16) and eddy potential energy, which were an order of magnitude larger near strong western boundary currents and their eastward extensions than in the interior of the ocean. Holland and Rhines concluded that the abyssal layer dynamics were primarily eddy-driven and acted similar to the down-gradient diffusion of potential vorticity. In the upper layer, the same parameterization held true near the jet separation point and in the region of westward recirculations.

Some analytical models have taken into account the fact that the observed transport of the Gulf Stream is several times larger than that given by the lin- 


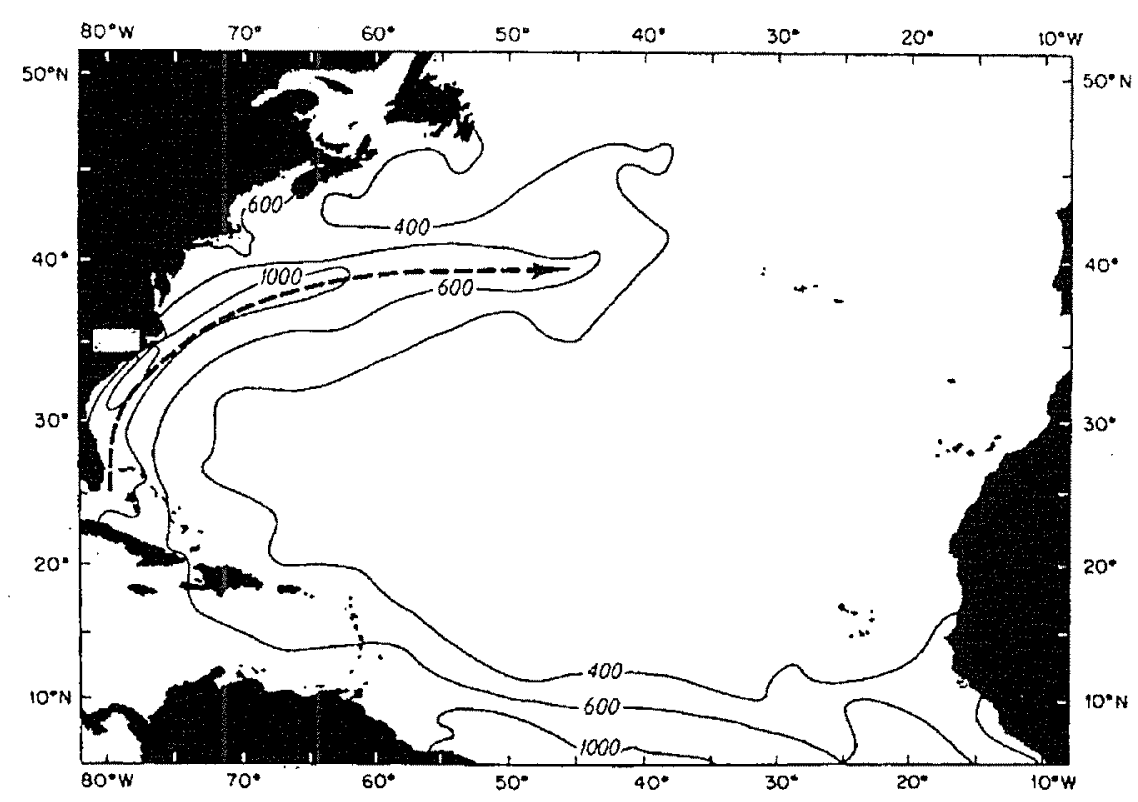

Figure 1-6: Surface eddy kinetic energy per unit mass in $\mathrm{cm}^{2} \mathrm{~s}^{-2}$ for the North Atlantic Ocean on a $1^{\circ}$ grid. Adapted from Schmitz et al. [37].

ear Sverdrup balance and therefore have focused on strongly nonlinear dynamical regimes. The recirculations were given as a steady, inertial, almost free (forcing and dissipation were thought to be negligible to the leading order) solution of the quasigeostrophic equations. In both barotropic (Cessi et al. [5]) and baroclinic (Marshall and Nurser [24]; Greatebatch [9]; Cessi [6]) models, eddies were parameterized as lateral down-gradient diffusion of potential vorticity and were shown to play crucial role in generating the recirculating gyres.

Jayne et al. [18] studied recirculations emerging due to both the internal dynamics of the jet and the inertial forcing. A quasigeostrophic barotropic model was forced by an unstable eastward jet inflow on the western boundary and a stabilized eastward jet outflow on the eastern boundary. The resulting barotropic eddies produced regions of homogenized potential vorticity north and south of the jet, giving rise to recirculations. Their strength was found to be a monotonically decreasing function of nondimensional $\beta$. However, it was concluded that in the sense of "eddy 
Sverdrup relation", $\beta \bar{v}=-\nabla \cdot \overline{\mathbf{u}^{\prime} q^{\prime}}$, the recirculations were predominantly inertial currents and only weakly forced by the eddies.

Therefore, the results of numerical experiments and the observational data suggest strong correlation between the dynamics of the Gulf Stream, its recirculations and the western boundary current. In the first part of the thesis, we study the problem of the development of the recirculations during free spin down of an unstable, zonally symmetric, quasi-geostrophic jet. The following questions are addressed:

- Can recirculations develop in a zonally symmetric unstable jet? If so, what is the basic mechanism driving the recirculations?

- How do the jet strength and structure influence recirculation characteristics?

- What are the differences in the equilibrated structure for baroclinic and barotropic jets?

Zonal symmetry combined with the absence of any forcing excludes the possibility of closing of the potential vorticity contours which may lead to the recirculation development. Hence, eddies generated by the instabilities if the jet provide the only mechanism for the changes in the mean flow structure and therefore for the generation of recirculations.

Many time-dependent wind-driven models have exhibited intrinsic low frequency variability that is often associated with the existence of multiple dynamical regimes. For example, in a reduced-gravity, free slip, quasi-geostrophic model with interfacial friction and biharmonic diffusion, McCalpin and Haidvogel [25] observed chaotic transitions between three different regimes (Figure 1-7): a high energy state with a strong, straight and deep penetrating eastward jet, a low energy state with a weakly penetrating jet and violent meanders and eddies, and a medium energy state with intermediate jet penetration and some eddy/meander formation. It was hypothesized that variations in the eddy-western boundary current interaction provided a 

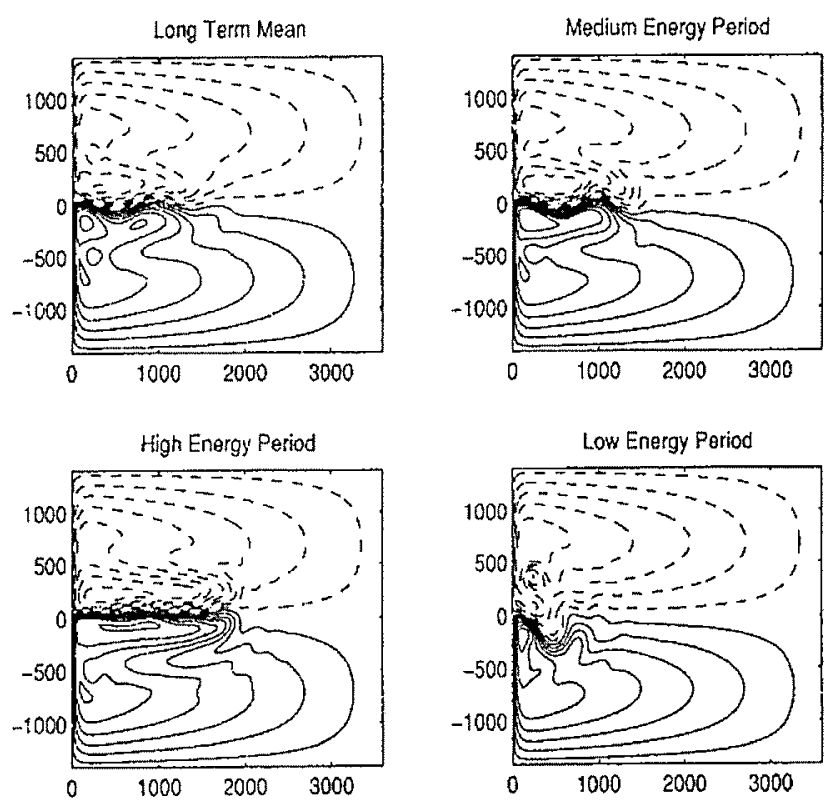

Figure 1-7: Contours of the time-averaged interface anomaly field for high energy period (lower left), low energy period (lower right), and medium energy period (upper right). Adapted from McCalpin and Haidvogel [25].

mechanism for the persistence of different regimes and the transitions between them. Similar high and low energy regimes were observed by Spall [39] in a wind-forced primitive equation model with bottom friction and lateral diffusion and with open boundary conditions. The presence of the Deep Western Boundary Current and its interaction with the upper layer wind-driven current were crucial for the existence of multiple dynamical regimes. A number of wind-driven, two-layer, quasi-geostrophic models also showed multiple dynamical regimes, such as those of Meacham (personal communication) and Berloff and McWilliams [4].

However, in the wind-driven models, is it hard to distinguish between two possible mechanisms affecting low frequency variability: the global structure of the wind forcing and the internal dynamics of an unstable jet. In the present study, we isolate the latter as the mechanism responsible for low frequency variability and address the following questions: 
- Are low frequency variability and multiple dynamical regimes intrinsic parts of the internal dynamics of a quasi-geostrophic jet and its recirculations?

- Are there any differences between barotropic and baroclinic instabilities as mechanisms affecting low frequency variability?

- Which dynamical regimes are associated with low frequency variability and which processes are responsible for the transitions between them?

- Are there any differences between low frequency variability in the globally forced and boundary-forced models?

\subsection{Outline of the Thesis}

This thesis studying the problems of generation and maintenance of recirculations by the Gulf Stream instabilities consists of two parts. The first part investigates the development of recirculations during the spin down of a quasi-geostrophic, zonally symmetric, unstable jet. The second part of the thesis examines the problem of existence and maintenance of low frequency variability and of multiple dynamical regimes due to the mechanisms of barotropic and baroclinic instabilities.

Chapter 2 addresses the first problem in a framework of the reduced-gravity and the two-layer quasi-geostrophic, doubly-periodic, zonally symmetric models. It examines the following questions: what is the basic mechanism for driving the westward flows; how do the jet strength and structure influence recirculation characteristics; what are the differences in the equilibrated structure for the baroclinic and barotropic jets? The chapter begins with the formulation of the numerical model and is followed by the discussion of the results of a time-dependent two-layer case. The strength and the structure of the recirculations, arising due to eddy heat and momentum fluxes, are examined for different values of nondimensional $\beta$-parameter and for a set of basic flows with varying degree of baroclinicity. To study the nature of 
eddy-mean flow interaction and its role in recirculation generation, a linear stability analysis is performed for basic flows with different degrees of baroclinicity and various jet widths. A hierarchy of "mean-flow" models, which includes only a mechanism of eddy-mean flow interactions, is further examined. The formulation and the numerical results of the time-dependent reduced-gravity model and a corresponding linear stability analysis follow the discussion.

Chapters 3 introduces a two-layer, quasi-geostrophic, colliding jets model, which is forced by the inflows and outflows through the open boundaries. Such forcing allows the isolation of barotropic and baroclinic instabilities as the mechanisms responsible for multiple dynamical states and low frequency variability. The chapter begins with the description of model equations, initial and boundary conditions, frictional operators and dimensional and nondimensional parameters. The importance of local forcing and its difference from the global wind forcing are illustrated in a section on model energetics. The dynamics of rings on the western boundary are discussed at the end of the chapter.

Chapter 4 reports the results of the reduced-gravity, colliding jets model, which isolates the importance of barotropic instability and dissipation for low frequency variability. Following the model formulation, the antisymmetric steady solutions and their linear stability properties are presented for various values of the interfacial drag coefficient. The chapter further focuses on the sensitivity of the time-dependent solutions to changes in biharmonic diffusion and interfacial friction. A detailed description of a reference run shows that the model has two preferred energy states, each associated with a unique dynamical regime. The discussion of the dynamical differences between the states and their transitions as well as a comparison with the results of the wind-forced models follow.

Chapter 5 addresses a problem of low frequency variability and multiple dynamical states in a framework of a two-layer, colliding jets model, which includes both baroclinic and barotropic instability mechanisms. The sensitivity of the model 
to changes in the value of the bottom friction parameter is examined and is related to a scaling analysis. The discussion of a reference run includes the analysis of energy time series, an extended empirical orthogonal function decomposition for low- and high-frequency variability and a diagnosis of zones of baroclinic growth. The results are compared with those of the reduced-gravity, colliding jets model and a two-layer, wind-forced models.

Chapter 6 presents conclusions for the Thesis, summarizes limitations of the present study, poses questions for further research and discusses relevance of this work to the real ocean. 


\section{Chapter 2}

\section{Generation of Recirculations in a Two-Layer Quasi-Geostrophic Zonally Symmetric Jet Model}

The present Chapter addresses the following questions:

- Can recirculations develop in a zonally symmetric unstable jet? If so, what is the basic mechanism driving the recirculations?

- How do the jet strength and structure influence recirculation characteristics?

- What are the differences in the equilibrated structure for baroclinic and barotropic jets? 


\subsection{Model Formulation}

\subsubsection{Model Equations}

A time-dependent, two-layer, quasi-geostrophic, semi-spectral model is integrated in a square, doubly periodic domain. The governing potential vorticity equations are

$$
\left(\frac{\partial}{\partial t}+\frac{\partial \psi_{i}}{\partial x} \frac{\partial}{\partial y}-\frac{\partial \psi_{i}}{\partial y} \frac{\partial}{\partial x}\right) q_{i}+\beta \frac{\partial \psi_{i}}{\partial x}=\mathcal{F}+\mathcal{S}_{i}
$$

where $q_{i}=\nabla^{2} \psi_{i}-F_{i}\left(\psi_{i}-\psi_{3-i}\right), F_{i}=f_{0}^{2} L^{2} / g^{\prime} D_{i}, \beta=\beta_{\text {dim }} L^{2} / U$ and $i=1$, 2. Since we are interested in the problem of a nonlinear equilibration of a quasi-geostrophic jet, the forcing is set to zero. However, a numerical filter, $\mathcal{F}$, is required for numerical stability; it acts to remove small scale enstrophy without dissipating too much energy. Because of the periodicity in the meridional direction, two sponge layers are set up near the northern and southern boundaries. In Equation (2.1), they are represented by a sponge operator $\mathcal{S}_{i}$. Sponges prevent any disturbance which leaves the domain through the northern (southern) boundary from reentering it from the south (north). More details on the form of the sponge operator are presented later. The numerical code is modified from a program by Glenn Flierl (personal communication).

\subsubsection{Dimensional Scales}

The dimensional scales of the model are chosen as follows. The horizontal length scale, $L$, is $407 \mathrm{~km}$. The model domain is square defined by $L_{\text {domain }} \times L_{\text {domain }}$, where $L_{\text {domain }}=2 \pi L=2560 \mathrm{~km}$. The upper and lower layer depths are $D_{1}=1 \mathrm{~km}$ and $D_{2}=4 \mathrm{~km}$ respectively. The baroclinic deformation radius, $L_{d e f}$, is $40 \mathrm{~km}$. The velocity scale, $U=0.8 \mathrm{~m} \mathrm{sec}^{-1}$, is chosen as the maximum velocity in the upper layer. The time scale is chosen as an advective time scale, $T_{a d v}=L / U=29.6$ days. The Coriolis parameter is represented by $f=f_{0}+\beta y$, where $f_{0}=10^{-4} \mathrm{sec}^{-1}$ and $\beta=2 \cdot 10^{-11} \mathrm{~m}^{-1} \mathrm{sec}^{-1}$. 


\subsubsection{Nondimensional Parameters}

The model is characterized by four nondimensional parameters:

- the ratio of the advective time scale to the barotropic Rossby wave period: $\beta=\beta_{\text {dim }} L^{2} / U$

- the inverse deformation radius, defined as the ratio of a horizontal length scale to the internal baroclinic deformation radius: $\gamma=\sqrt{F_{1}+F_{2}}=$ $L / L_{d e f}$

- the depth ratio: $\delta=D_{1} / D_{2}$;

- the ratio of the upper layer initial velocity amplitude, $u_{1}$, to the lower layer initial velocity amplitude, $u_{2}: \alpha=u_{1} / u_{2}$.

In all the experiments, the values of $\delta$ and $\gamma$ are fixed at 0.25 and 10.18 respectively and the values of $\beta$ and $\alpha$ are varied.

\subsubsection{Sponge Operator}

As was mentioned before, two sponge layers are set up near the northern and southern boundaries to remove periodicity in the meridional direction. After performing a series of experiments with different widths of the sponge layer, we found the optimal width of about $300 \mathrm{~km}$, which allows enough distance for the decay of the disturbances. Whenever a sponge layer was too narrow, it acted as a reflector rather than an absorber.

The sponge operator has the following form:

$$
\mathcal{S}_{i}=-\mathcal{R}(y)\left(q_{i}-\beta y\right)-\frac{\partial \mathcal{R}}{\partial y} \frac{\partial \psi_{i}}{\partial y} .
$$

The first term is represented by a sponge function, $\mathcal{R}$ (Figure 2-1), acting to relax the potential vorticity field to the planetary vorticity. This type of damping is similar 


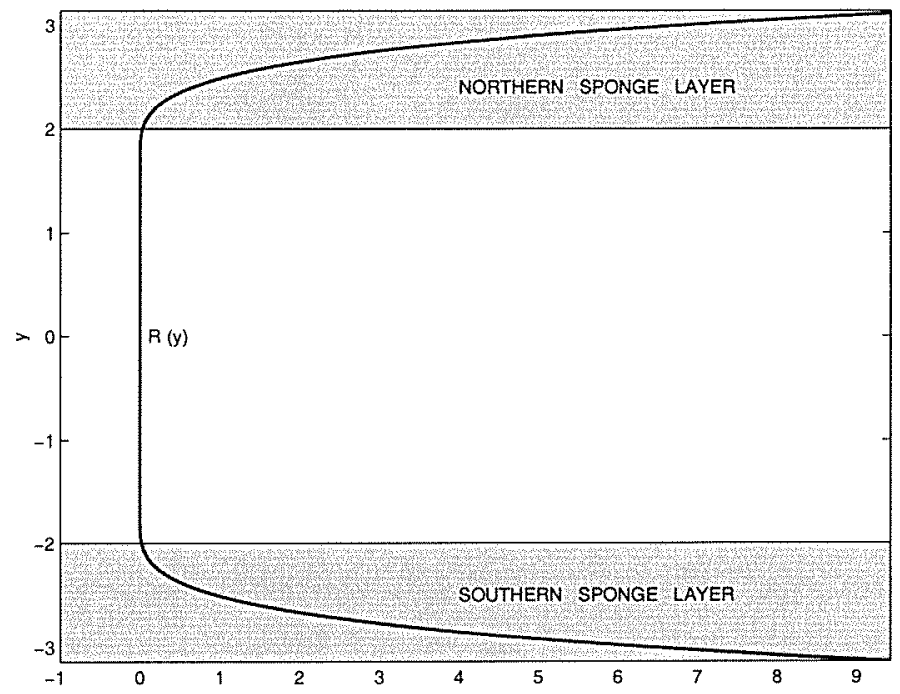

Figure 2-1: Sponge function, $\mathcal{R}$, defines the ratio of the advective time scale to the decay time scale. The latter varies from being infinite in the interior of the domain to 0.5 day near the boundary.

to bottom friction. The meridionally dependent friction coefficient, $\mathcal{R}(y)$, is the ratio of the advective time scale to the decay time scale, $T_{a d v} / T_{\text {decay }}$. Within each sponge layer, the decay time scale, $T_{\text {decay }}$, slowly decreases from being infinite to 0.5 day at the boundary.

The second term in the sponge operator involves the meridional gradient of a sponge function acting on the velocity field. It helps to remove spurious energy arising from boundary currents with zero vorticity. This term comes naturally from the following velocity damping equations:

$$
\frac{D \mathbf{u}_{\mathbf{i}}}{D t}=-\mathcal{R}(x, y)\left(\mathbf{u}_{i}-\mathbf{U}_{i}\right), i=1,2
$$

where $\mathbf{u}_{i}=\left(u_{i}, v_{i}\right)^{T}$ is a horizontal velocity vector, $\mathbf{U}_{i}=\left(U_{i}, V_{i}\right)^{T}$ is a relaxation velocity vector and $\mathcal{R}$ is a friction coefficient, which in general could be a function of spatial variables. The equation for relative vorticity, $\zeta_{i}=\frac{\partial v_{i}}{\partial x}-\frac{\partial u_{i}}{\partial y}$, becomes

$$
\frac{D \zeta_{\mathbf{i}}}{D t}=-\mathcal{R} \zeta_{i}-\frac{\partial \mathcal{R}}{\partial x} v_{i}+\frac{\partial \mathcal{R}}{\partial y} u_{i}
$$


Zonal symmetry in the model implies that the frictional coefficient changes only in the meridional direction, i.e. $\frac{\partial \mathcal{R}}{\partial x}=0$. The relaxation velocity, $\mathbf{U}_{\mathbf{i}}$, was chosen to be zero.

\subsubsection{Basic Flow}

The basic velocity profile corresponds to a thin eastward jet and is defined as

$$
U_{i}(y)=-\frac{\partial \Psi(y)}{\partial y}=\frac{u_{i}}{\cosh ^{2} w y}, i=1,2
$$

The amplitude of the lower layer nondimensional velocity, $u_{2}$, varies from being zero to the amplitude of the upper layer nondimensional velocity, $u_{1}$. Thus, we can create both strongly baroclinic jets or purely barotropic jets (Figure 2-2a). The parameter $w$ defines the width of the jet and is chosen to equal 8.14, which corresponds to a 100 $\mathrm{km}$ wide basic flow.

The basic potential vorticity is then given by

$$
Q_{i}=\beta y+\frac{\partial^{2} \Psi_{i}}{\partial y^{2}}+F_{i}\left(\Psi_{j}-\Psi_{i}\right)=\beta y+\tanh w y\left\{\frac{2 u_{i} w}{\cosh ^{2} w y}+\frac{F_{i}}{w}\left(u_{j}-u_{i}\right)\right\}
$$

The basic potential vorticity gradient changes sign, so that it satisfies the necessary condition for instability (Figure 2-2b). The results of the linear stability analysis are presented later.

\subsubsection{Initialization}

We perturb the basic flow given by Equation (2.5) with a sum of wave packets of different wave lengths, each having a Gaussian envelope. Initially, the disturbance is localized in the middle of the domain.

\subsection{Linear Stability Analysis of a Two-Layer Model}

Two processes play major roles in a nonlinear spin down of an unstable quasigeostrophic jet: eddy-mean flow and eddy-eddy interactions. The following section 
(a)

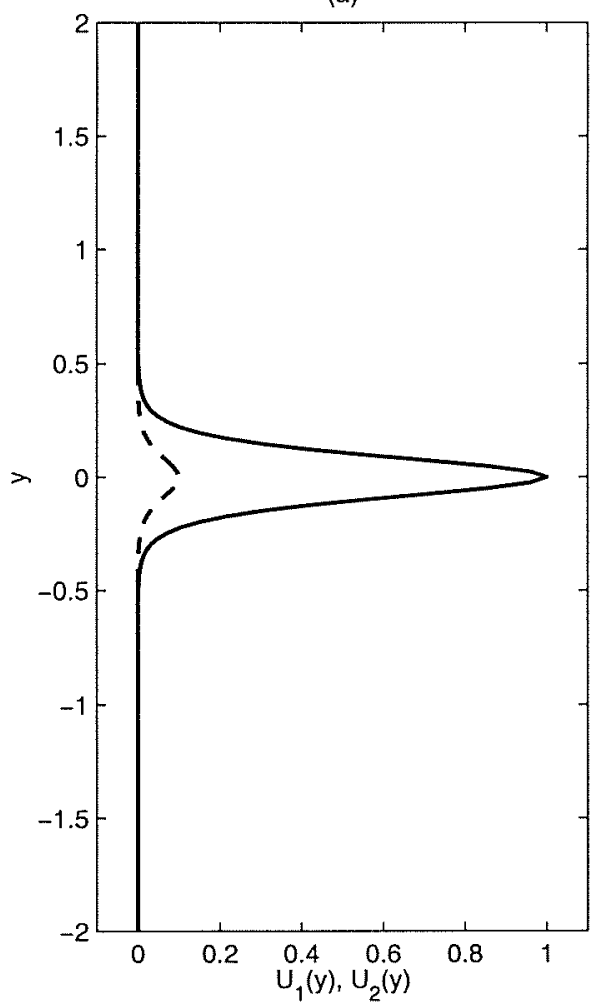

(b)

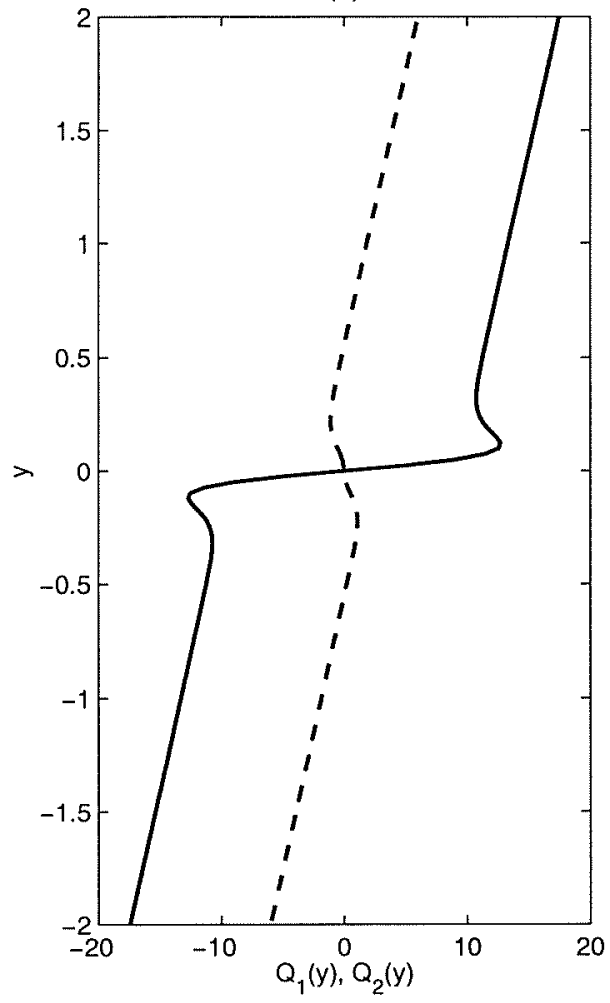

Figure 2-2: The nondimensional velocity (a) and potential vorticity (b) for the upper layer (solid line) and lower layer (dash line) initial basic flow. Parameters: $u_{1}=$ $1, u_{2}=0.1, w=8.14, \beta=4.15, \gamma=10.18, \delta=0.25, U=0.8 \mathrm{~m} \mathrm{sec}^{-1}$. North-south extent of the full domain is $[-\pi, \pi]$.

reports the results of a linear stability analysis which studies the influence of the barotropic and baroclinic eddy field on the mean flow structure.

Although the nonlinear model defined by Equation (2.1) has a numerical frictional operator and sponge layers near the doubly-periodic meridional boundaries, the linearized model is solved for an inviscid case in a channel. Such simplifications are possible for two reasons. First, in the nonlinear model, friction is very weak. Second, as we show later, unstable normal modes resulting from the linear stability analysis are zero in the regions where a sponge operator of the nonlinear model is not trivial. 


\subsubsection{Linear Model Formulation}

Linearizing the inviscid quasi-geostrophic equations of motion (2.1) around a basic flow gives:

$$
\frac{\partial q_{i}}{\partial t}+\mathcal{J}\left(\Psi_{i}, q_{i}\right)+\mathcal{J}\left(\phi_{i}, Q_{i}\right)=0
$$

where $\Psi_{i}(y)=-\int^{y} U_{i}\left(y^{\prime}\right) d y^{\prime}$ and $Q_{i}(y)=\beta y+F_{i}\left(\Psi_{3-i}-\Psi_{i}\right)+\frac{d^{2} \Psi_{i}}{d y^{2}}$ are the basic flow streamfunction and potential vorticity fields, $\phi_{i}=\phi_{i}(x, y, t)$ and $q_{i}=q_{i}(x, y, t)$ are the perturbation streamfunction and potential vorticity fields and $i=1,2$ is a layer index. We look for a zonally periodic solution of the form

$$
\phi_{i}(x, y, t)=\operatorname{Real}\left(\Phi_{i}(y) e^{i k(x-c t)}\right),
$$

where $k$ is a horizontal wave number and $c$ is the phase speed of the disturbance. Substituting such a solution into Equation (2.7) gives the following problem for the normal mode meridional structure:

$$
\left(U_{i}(y)-c\right)\left(\frac{d^{2} \Phi_{i}}{d y^{2}}-k^{2} \Phi_{i}+F_{i}\left(\Phi_{3-i}-\Phi_{i}\right)\right)+\Phi_{i} \frac{\partial Q_{i}}{\partial y}=0, i=1,2
$$

subject to no-normal-flow boundary conditions on the lateral boundaries

$$
\Phi_{i}(y=\pi)=\Phi_{i}(y=-\pi)=0 .
$$

\subsubsection{Method of Solution}

The linear problem (2.9) is solved numerically. Discretizing the equations at $y^{j}=$ $-\pi+\Delta y(j-1)$, where $\Delta y=\frac{2 \pi}{N-1}$ and $j=1, \ldots, N$, using a second order finite difference scheme and incorporating boundary conditions (2.10), provides a generalized eigenvalue problem of the form:

$$
A \Phi=c B \Phi
$$

Matrices $A$ and $B$ have the following structures:

$$
A=\left[\begin{array}{cc}
\mathcal{H}_{1} & \mathcal{G}_{2} \\
\mathcal{G}_{1} & \mathcal{H}_{2}
\end{array}\right]
$$




$$
B=\left[\begin{array}{ll}
\mathcal{P}_{1} & \mathcal{Z}_{2} \\
\mathcal{Z}_{1} & \mathcal{P}_{2}
\end{array}\right],
$$

where $\mathcal{H}_{i}$ and $\mathcal{P}_{i}$ are tri-diagonal matrices defined as

$$
\mathcal{H}_{i}=\left[\begin{array}{lllllll}
h_{i}^{(2)} & U_{i}^{(2)} & 0 & 0 & \cdots & 0 & 0 \\
U_{i}^{(3)} & h_{i}^{(3)} & U_{i}^{(3)} & 0 & \cdots & 0 & 0 \\
0 & U_{i}^{(4)} & h_{i}^{(4)} & U_{i}^{(4)} & \cdots & 0 & 0 \\
\vdots & & & & & & \\
0 & 0 & 0 & 0 & \cdots & h_{i}^{(N-2)} & U_{i}^{(N-2)} \\
0 & 0 & 0 & 0 & \cdots & U_{i}^{(N-1)} & h_{i}^{(N-1)}
\end{array}\right]
$$

with diagonal elements

$$
\begin{gathered}
h_{i}^{(j)}=U_{i}^{(j)}\left(-2-\Delta y^{2}\left(k^{2}+F_{i}\right)\right)+\Delta y^{2}{\frac{\partial Q_{i}}{\partial y}}^{(j)}, \\
U_{i}^{(j)}=U_{i}\left(y=y^{j}\right),
\end{gathered}
$$

and

$$
\mathcal{P}_{i}=\left[\begin{array}{lllllll}
p_{i}^{(2)} & 1 & 0 & 0 & \cdots & 0 & 0 \\
1 & p_{i}^{(3)} & 1 & 0 & \cdots & 0 & 0 \\
0 & 1 & p_{i}^{(4)} & 1 & \cdots & 0 & 0 \\
\vdots & & & & & & \\
0 & 0 & 0 & 0 & \cdots & p_{i}^{(N-2)} & 1 \\
0 & 0 & 0 & 0 & \cdots & 1 & p_{i}^{(N-1)}
\end{array}\right]
$$

with diagonal elements

$$
p_{i}^{(j)}=-2-\Delta y^{2}\left(k^{2}+F_{i}\right)
$$

for $i=1,2$ and $j=2, \cdots, N-1$. Matrices $\mathcal{G}_{i}$ and $\mathcal{Z}_{i}$ are diagonal with nonzero elements equal $g_{i}^{(j)}=U_{i}^{(j)} F_{i} \Delta y^{2}$ and $z_{i}^{(j)}=F_{i} \Delta y^{2}$ respectively.

MATLAB's SPTARN routine was used to solve the generalized eigenvalue problem defined in Equation (2.11). We performed a series of experiments with 

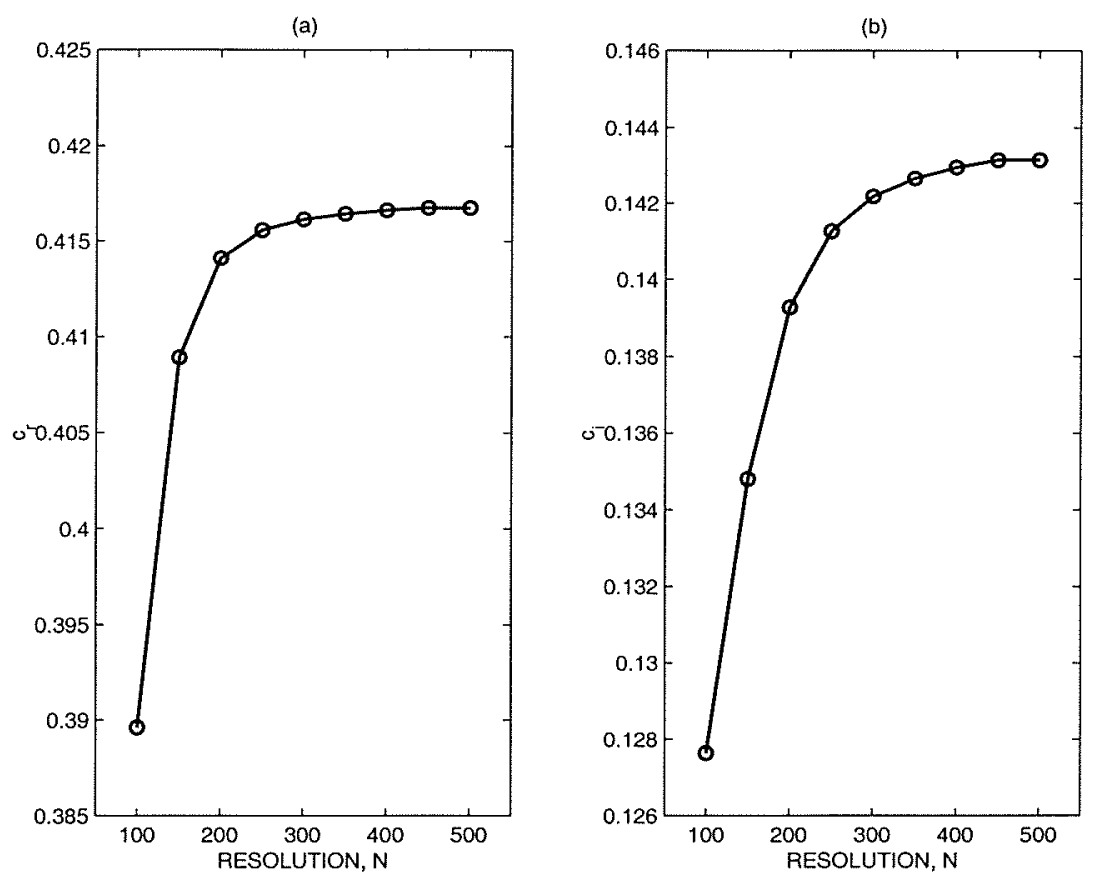

Figure 2-3: Real, $c_{r},(\mathrm{a})$ and imaginary, $c_{i}$, (b) parts of an eigenvalue as functions of resolution, $\mathrm{N}$, for $u_{1}=1, u_{2}=0.99, \beta=4.15, \gamma=10.18, \delta=0.25, k=8, U=0.8$ $\mathrm{m} \mathrm{sec}^{-1}$.

different resolutions to ensure sufficient convergence. Figure 2-3 shows the real and imaginary parts of an eigenvalue as functions of resolution, $N$, when $u_{1}=1, u_{2}=$ $0.99, \beta=4.15, \gamma=10.18$, and $k=8 . N=500$ was found to be sufficient for the choice of parameters considered in the numerical experiments.

\subsubsection{Maximum Growth Rates of Unstable Modes}

The first set of experiments was performed for the basic flow with the following parameters: $u_{1}=1, \beta=4.15, \gamma=10.18, \delta=0.25$ and the lower layer basic velocity amplitude $u_{2}$ varying between 0 and 0.99 . Typical unstable eigenvalues are presented in Figure 2-4. As $k$ increases, the number of unstable modes decreases from three to zero. Long waves are weakly unstable. Notice that the linear stability analysis was performed only for the integer horizontal wave numbers, $k=1,2, \ldots, n$. The reason 
is that only waves with such wave numbers are present in the corresponding fully nonlinear doubly-periodic model.

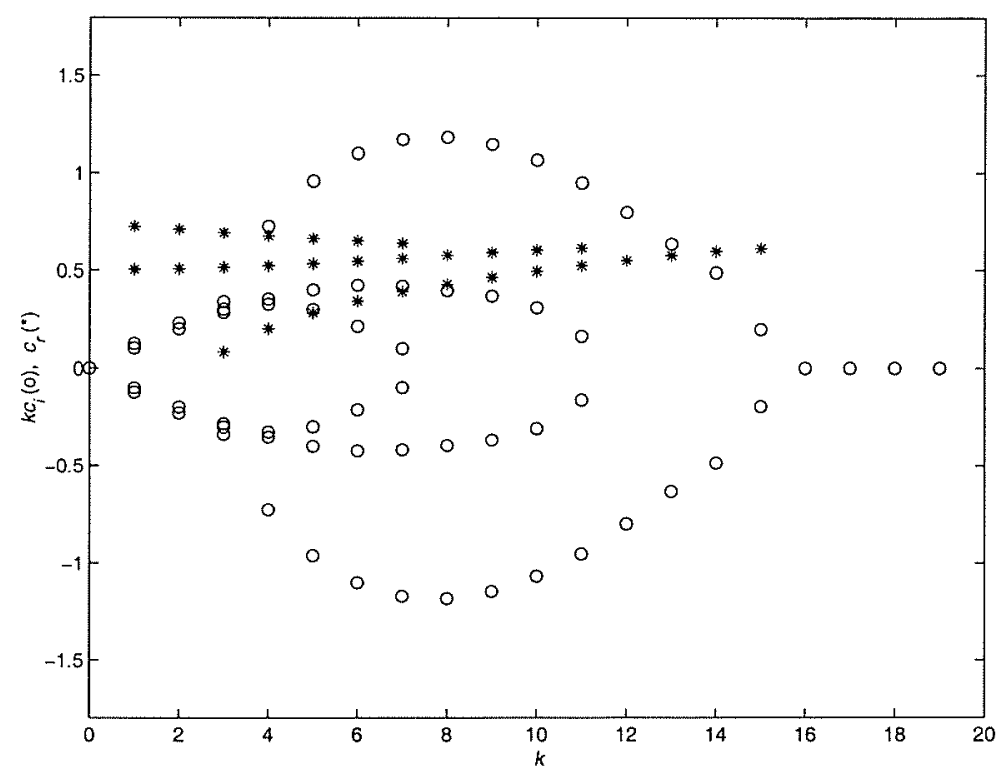

Figure 2-4: The growth rates, $k c_{i},(0)$ and the corresponding real parts of the phase speed, $c_{r},\left(^{*}\right)$ as functions of a horizontal wave number, $k$. Parameters: $u_{1}=1, u_{2}=$ $0.99, \beta=4.15, \gamma=10.18, \delta=0.25, U=0.8 \mathrm{~m} \mathrm{sec}^{-1}$.

Figure 2-5 shows the maximum growth rate, $k c_{i}$, as a function of a horizontal wave number, $k$. In this experiment, the amplitude of the upper layer nondimensional velocity, $u_{1}$, is fixed at 1.0. The amplitude of the lower layer nondimensional velocity, $u_{2}$, varies between 0.0 and 0.99 . Clearly, the maximum growth rate is not a monotonic function of $u_{2}$ (Figure 2-6); as the flow becomes more barotropic, it first decreases, reaching a minimum at $u_{2}=0.2$, and then increases, reaching a maximum at $u_{2}=0.99$. This non-monotonic behavior suggests that it is the baroclinic mode that dominates linear growth for $u_{2}<0.2$. As the barotropicity of the basic flow increases, the barotropic mode becomes more and more important, and finally it dominates the linear growth.

The dimensional length of the most unstable wave increases from $320 \mathrm{~km}$ $(k=6)$ to $426 \mathrm{~km}(k=8)$ with a decrease in baroclinicity. Correspondingly, the 

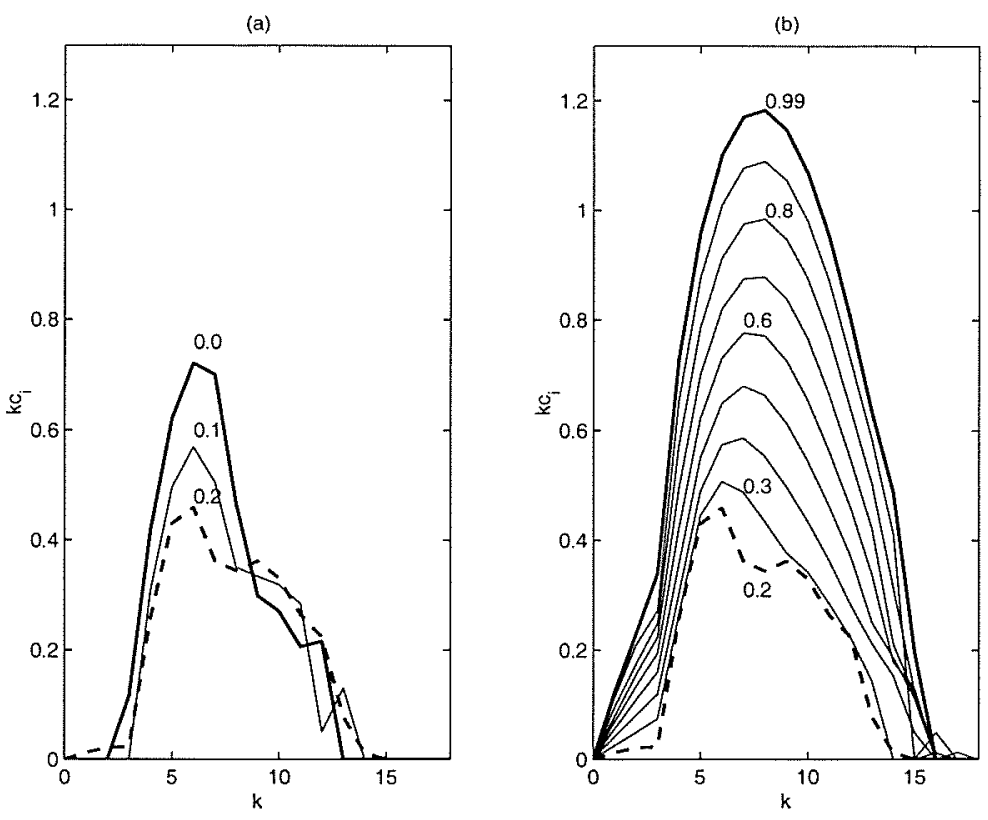

Figure 2-5: The maximum growth rate, $k c_{i}$, as a function of a horizontal wave number, $k$. The amplitude of the upper layer nondimensional basic velocity, $u_{1}$, is 1 . The amplitude of the lower layer nondimensional basic velocity, $u_{2}$, changes from 0 to 0.2 (a) and from 0.2 to 0.99 (b). Parameters: $\beta=4.15, \gamma=10.18, \delta=0.25, U=0.8 \mathrm{~m}$ $\sec ^{-1}$.

dimensional phase speed changes from $0.07 \mathrm{~m} \mathrm{sec}^{-1}$ to $0.34 \mathrm{~m} \mathrm{sec}^{-1}$. Independent of baroclinicity, the flow is unstable for $0<k<15$, so that only waves shorter than 170 $\mathrm{km}$ are stable.

The meridional structure of the most unstable mode is similar for all considered values of the lower layer nondimensional velocity amplitude, $u_{2}$ (Figure 2-7). The mode is nonzero only in the interior of the domain, which justifies the use of the linear model in a periodic channel and neglecting of the sponge layers.

Nondimensional $\beta$ has a stabilizing effect on the mean flow (Figure 2-8). For strongly baroclinic cases (a), waves longer that $900 \mathrm{~km}$ are linearly stable when $\beta=$ 6.64. The long wave cutoff disappears for smaller $\beta$. For barotropic basic flows (b), there is no long wave cutoff. Waves shorter than $160 \mathrm{~km}$ are stable. 


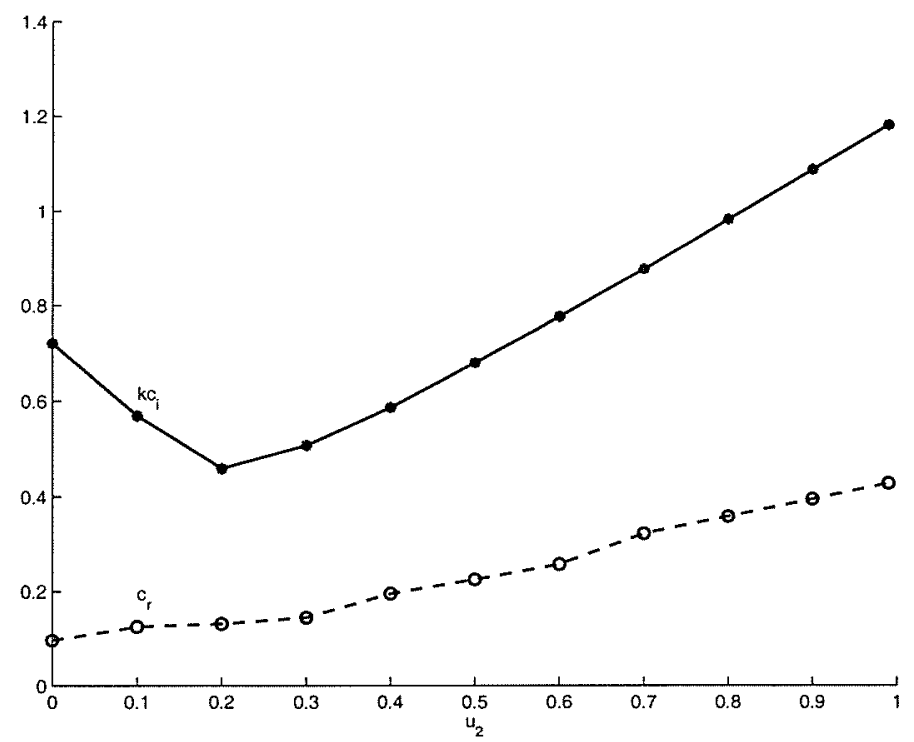

Figure 2-6: The maximum growth rate, $k c_{i}$, (solid line) and the corresponding nondimensional phase speed, $c_{r}$, (dashed line) as functions of the amplitude of the lower layer nondimensional basic velocity, $u_{2}$. Parameters: $u_{1}=1.0, \beta=4.15, \gamma=$ $10.18, \delta=0.25, U=0.8 \mathrm{~m} \mathrm{sec}^{-1}$.

\subsubsection{Wave - Zonal Mean Flow Interactions}

The Eulerian zonal mean circulation is calculated to understand how the most linearly unstable mode affects the mean flow. We assume the amplitude of the linear mode to be small and take the zonal average of Equations (2.7). Repeating the arguments given by Shepherd [35] and adopting his notations imply the following problem:

$$
\frac{\partial}{\partial t}\left(\frac{d^{2} \bar{\psi}_{i}}{d y^{2}}+F_{i}\left(\bar{\psi}_{3-i}-\bar{\psi}_{i}\right)\right)=-\overline{\mathcal{J}\left(\phi_{i}, q_{i}\right)}, i=1,2
$$

subject to boundary conditions:

$$
\bar{\psi}_{i}=0 \text { at } y=-\pi, \pi
$$

where bar denotes zonally averaged fields, and $\phi$ and $q$ are the streamfunction and the potential vorticity fields of the most unstable mode. 

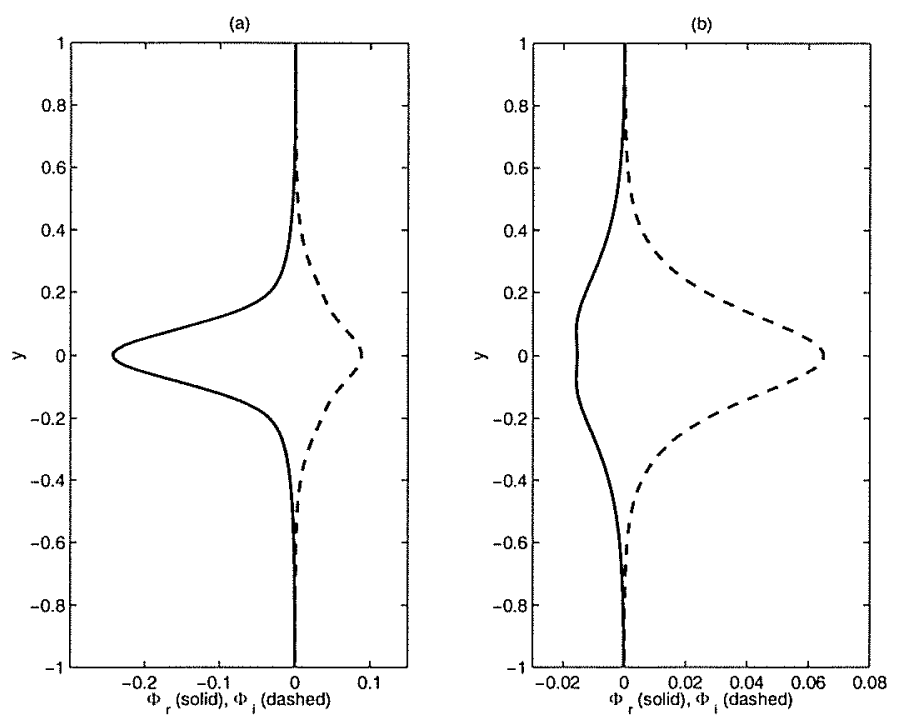

Figure 2-7: The real (solid line) and imaginary (dashed line) parts of the most unstable mode, $\Phi(y, k=6)$ in the upper layer (a) and in the lower layer (b). Parameters: $u_{1}=1.0, u_{2}=0.0, \beta=4.15, \gamma=10.18, \delta=0.25, U=0.8 \mathrm{~m} \mathrm{sec}^{-1}$.

Substituting a normal mode form into the forcing term, $\overline{\mathcal{J}\left(\phi_{i}, q_{i}\right)}$, of the above equations implies that the solution must obey the following:

$$
\frac{\partial \bar{\psi}_{i}(y, t)}{\partial t}=2 k c_{i} \Theta(y) e^{2 k c_{i} t}
$$

Thus, the problem (2.14) can be rewritten as

$$
\Theta_{i y y}+F_{i}\left(\Theta_{3-i}-\Theta_{i}\right)=-\frac{k}{4 k c_{i}} \operatorname{Im}\left(\phi_{i y}^{*} \phi_{i y y}-\phi_{i y y y}^{*} \phi_{i}+F_{i}\left(\phi_{3-i} \phi_{i y}^{*}-\phi_{(3-i) y}^{*} \phi_{i}\right)\right),(2
$$

where $i=1,2, j=3-i$ are layer indices, $k$ and $k c_{i}$ are the horizontal wave number and the growth rate of the most unstable mode and a star sign denotes complex conjugate. The boundary conditions are given by $\Theta(-\pi)=\Theta(\pi)=0$.

Finite differencing reduces problem (2.17) to solving $A \Theta=B$, where

$$
A=\left[\begin{array}{ll}
\mathcal{H}_{1} & \mathcal{G}_{2} \\
\mathcal{G}_{1} & \mathcal{H}_{2}
\end{array}\right] \text {. }
$$



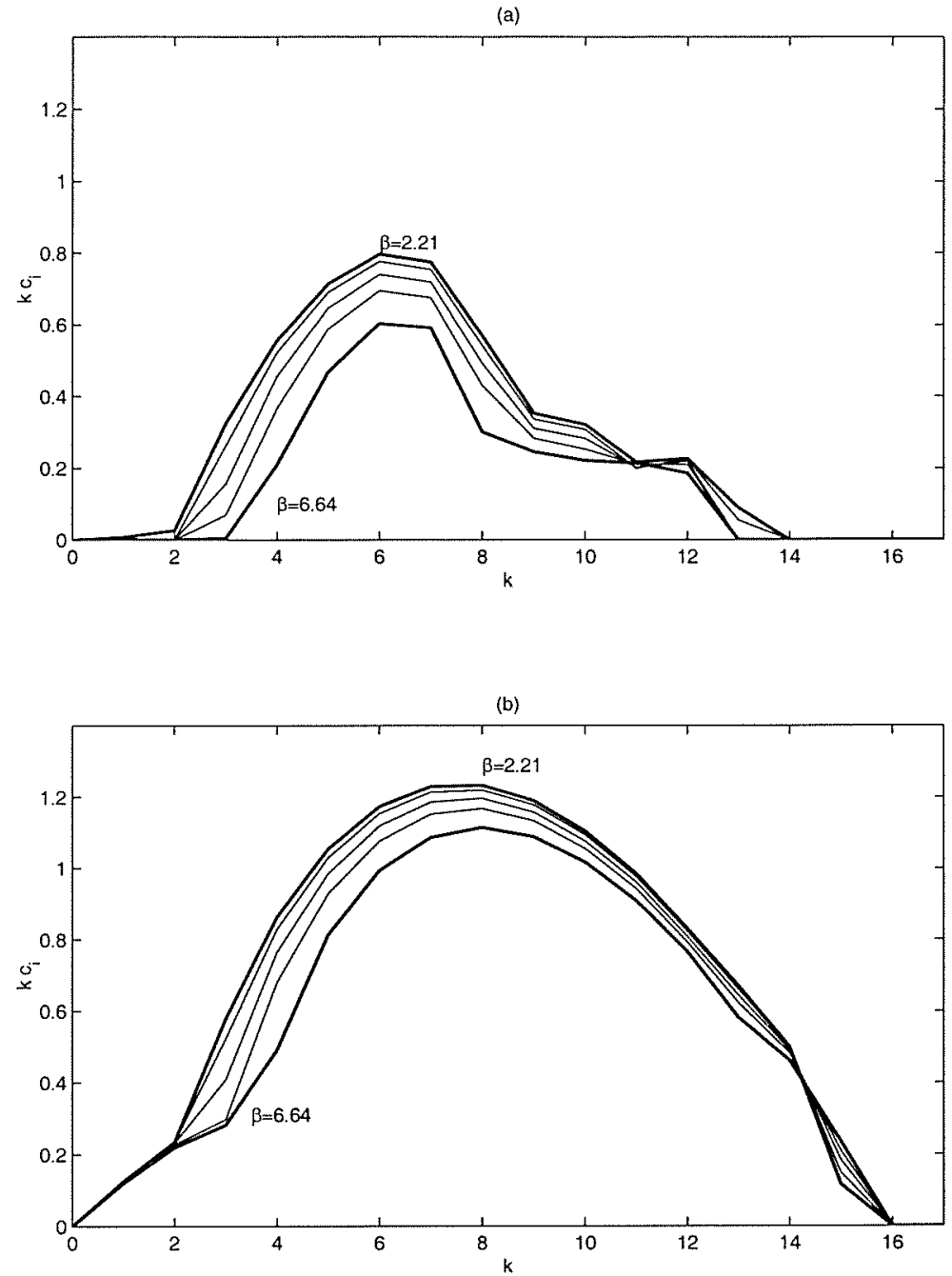

Figure 2-8: The maximum growth rate, $k c_{i}$, as a function of a horizontal wave number, $k$, for $\beta=(6.64,4.74,3.68,2.76,2.21)$. Parameters: $u_{1}=1, u_{2}=0$ in (a) and $u_{1}=$ $1, u_{2}=0.99$ in (b). 
Individual blocks of the matrix have the following structures:

$$
\mathcal{H}_{i}=\left[\begin{array}{cccccccc}
-3 & 4 & -1 & 0 & \cdots & 0 & 0 & 0 \\
1 & h_{i} & 1 & 0 & \cdots & 0 & 0 & 0 \\
0 & 1 & h_{i} & 1 & \cdots & 0 & 0 & 0 \\
0 & 0 & 1 & h_{i} & \cdots & 0 & 0 & 0 \\
\vdots & & & & & & & \\
0 & 0 & 0 & 0 & \cdots & 1 & h_{i} & 1 \\
0 & 0 & 0 & 0 & \cdots & 1 & -4 & 3
\end{array}\right]
$$

where $h_{i}=-2-\Delta y^{2} F_{i}$, and

$$
\mathcal{G}_{i}=\left[\begin{array}{cccccccc}
0 & 0 & 0 & 0 & \cdots & 0 & 0 & 0 \\
0 & g_{i} & 0 & 0 & \cdots & 0 & 0 & 0 \\
0 & 0 & g_{i} & 0 & \cdots & 0 & 0 & 0 \\
\vdots & & & & & & & \\
0 & 0 & 0 & 0 & \cdots & 0 & g_{i} & 0 \\
0 & 0 & 0 & 0 & \cdots & 0 & 0 & 0
\end{array}\right],
$$

where $g_{i}=\Delta y^{2} F_{i}, i=1,2$.

\subsubsection{Eddy Energy Equation}

Transient eddies can grow at the expense of kinetic or potential energy, or both, which implies barotropic, baroclinic or mixed nature of the instability process. The equation for eddy energy, $E^{\prime}$, (Pedlosky [27]) for the linear model is given by

$$
\frac{\partial}{\partial t} E^{\prime}=\int_{-\pi}^{\pi}\left(E K E C_{1}+E K E C_{2}+E P E C\right) d y,
$$

where the $i$-the layer eddy kinetic energy conversion, $E K E C_{i}$, is produced by the depth-weighted Reynolds stresses acting on the basic state shear:

$$
E K E C_{i}=\frac{D_{i}}{D} \overline{\frac{\partial \phi_{i}}{\partial x} \frac{\partial \phi_{i}}{\partial y}} \frac{\partial U_{i}}{\partial y}, i=1,2
$$


(a)

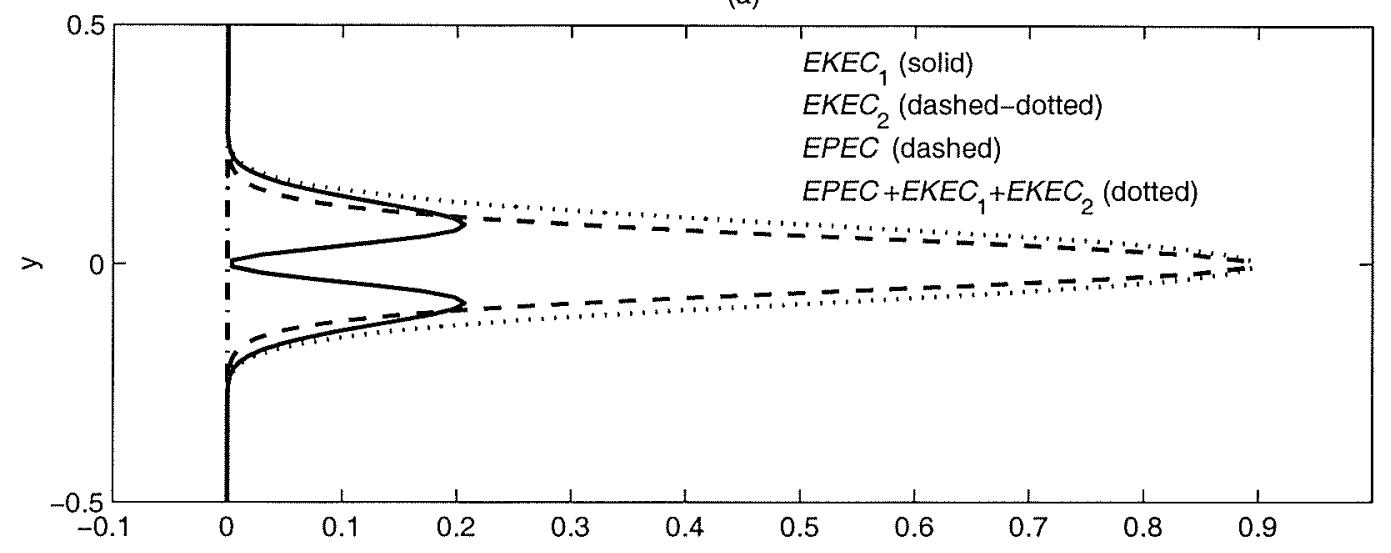

(b)

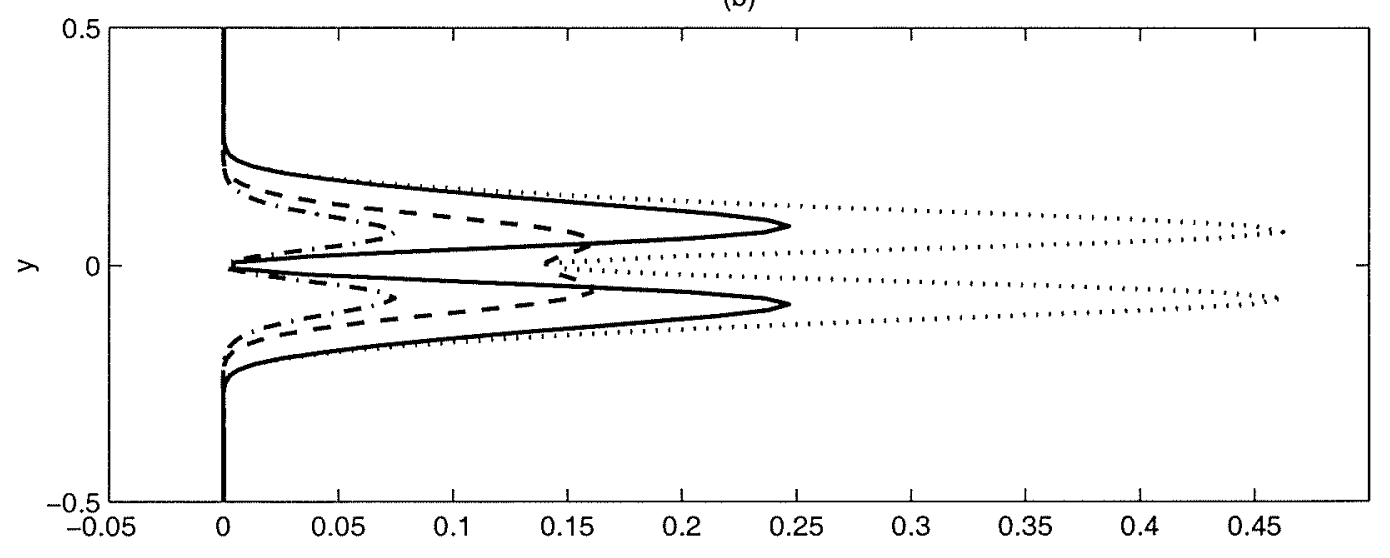

(c)

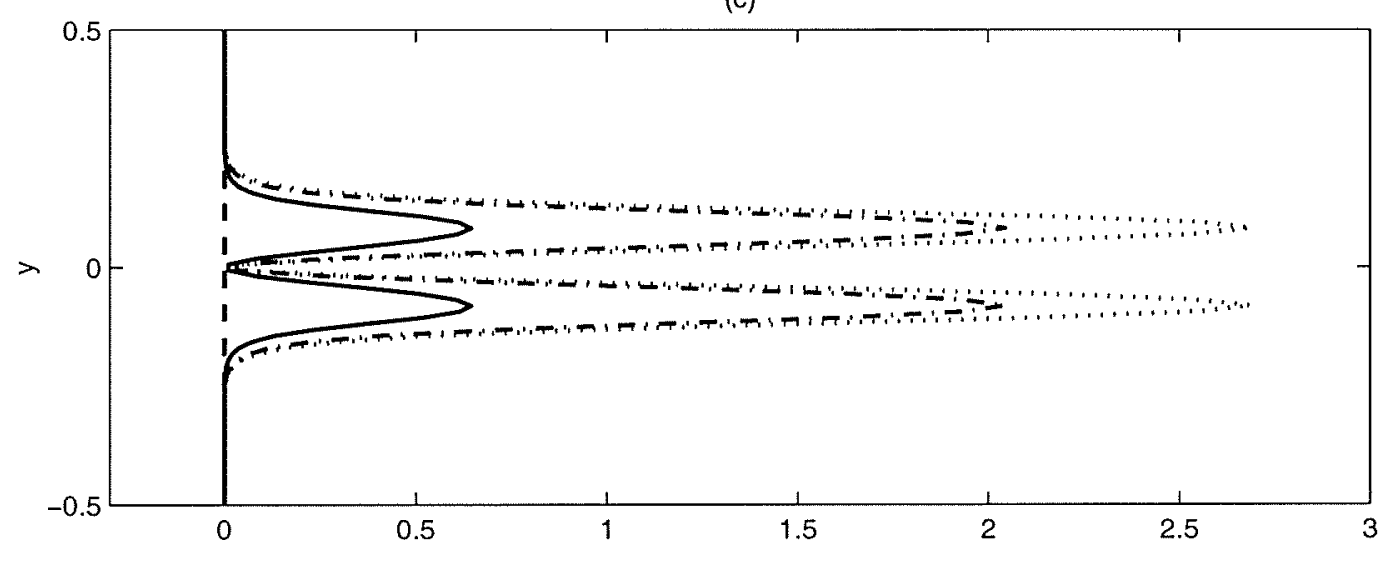

Figure 2-9: The eddy energy convergence terms: upper layer kinetic, $E K E C_{1}$, lower layer kinetic, $E K E C_{2}$, potential, $E P E C$, and total for $u_{2}=0$ (a), $u_{2}=0.2$ (b) and $u_{2}=0.99$ (c). Parameters: $u_{1}=1, \beta=4.15, \gamma=10.18, \delta=0.25, U=0.8 \mathrm{~m} \mathrm{sec}^{-1}$. 
and the eddy potential energy conversion, $E P E C$, is produced by the meridional eddy heat flux multiplied by the basic state temperature gradient:

$$
E P E C=\frac{F_{1} D_{1}+F_{2} D_{2}}{2 D}\left(U_{1}-U_{2}\right) \frac{\overline{\partial \phi_{2}}}{\partial x} \phi_{1} .
$$

When $u_{2}=0.99$ (Figure 2-9c), the instability is purely barotropic; the potential energy conversion is negligible. For $u_{2}=0.2$ (Figure 2-9b), the instability is mixed; both the kinetic and potential eddy energy conversion terms are positive and both participate equally in the growth in time of the total eddy energy. For the case of $u_{2}=0$ (Figure 2-9a), the instability is predominantly baroclinic with some barotropic contribution in the upper layer. In the lower layer, the eddy energy increases only due to the eddy heat fluxes. Thus, taking into account different nature of linear instability, we expect different impact of transient disturbances on the mean flow.

\subsubsection{Transformed Eulerian Mean Circulation}

To illuminate the dynamics of the linearly growing disturbance and its impact on the mean flow, the Transformed Eulerian Mean (TEM) circulation is calculated (Andrews and McIntyre [1], Pedlosky [27], Shepherd [35]):

$$
\frac{\partial \bar{u}_{i}}{\partial t}=\bar{v}_{i}^{*}+\nabla \cdot \mathbf{E P}_{i}, i=1,2 \text {. }
$$

In the TEM formulation, the time variations of the mean flow velocity result from the divergence of the Eliassen-Palm fluxes:

$$
\nabla \cdot \mathbf{E P}_{i}=\overline{v_{i} q_{i}}=-\frac{\partial}{\partial y}\left(\overline{u_{i} v_{i}}\right)+(-1)^{i} F_{i} \overline{v_{i}\left(\phi_{1}-\phi_{2}\right)}
$$

and from the residual meridional circulation:

$$
\overline{v_{i}^{*}}=\overline{v_{i}^{a}}-(-1)^{i} F_{i} \overline{v_{i}\left(\phi_{1}-\phi_{2}\right)}
$$

where $\overline{v_{i}^{a}}$ is an ageostrophic meridional velocity and $\left(u_{i}, v_{i}\right)^{T}=\left(-\frac{\partial \phi_{i}}{\partial y}, \frac{\partial \phi_{i}}{\partial x}\right)^{T}$ is a geostrophic perturbation velocity vector. 
(a)

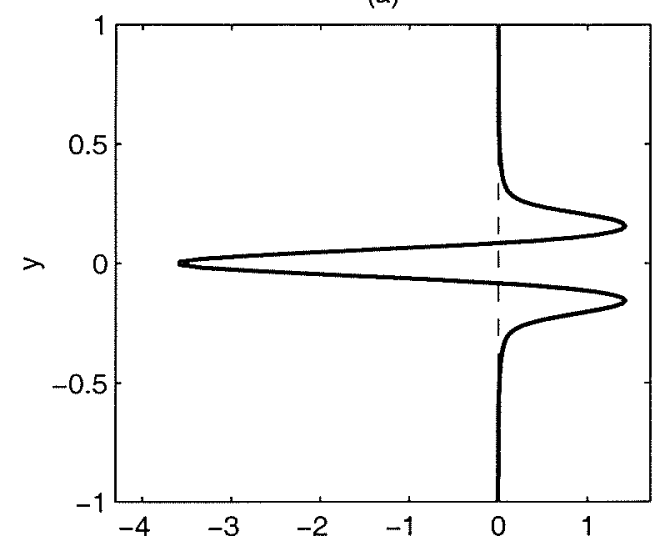

(c)

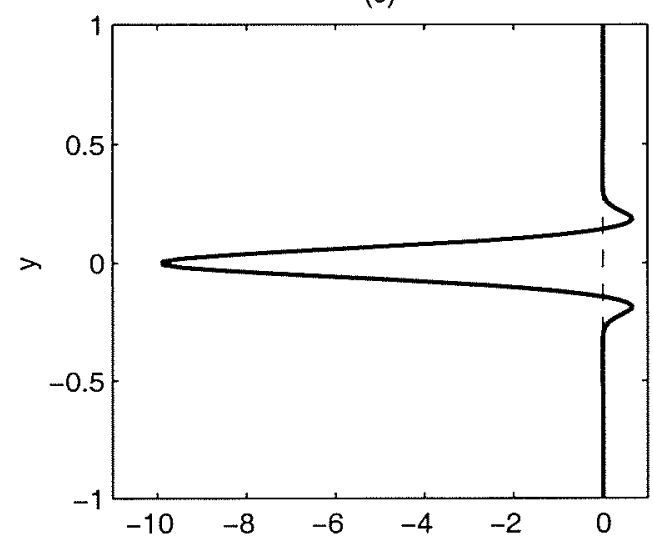

(e)

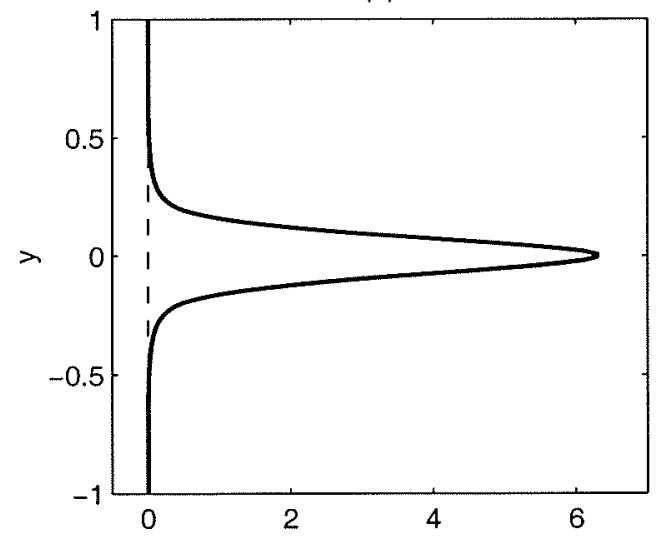

(b)

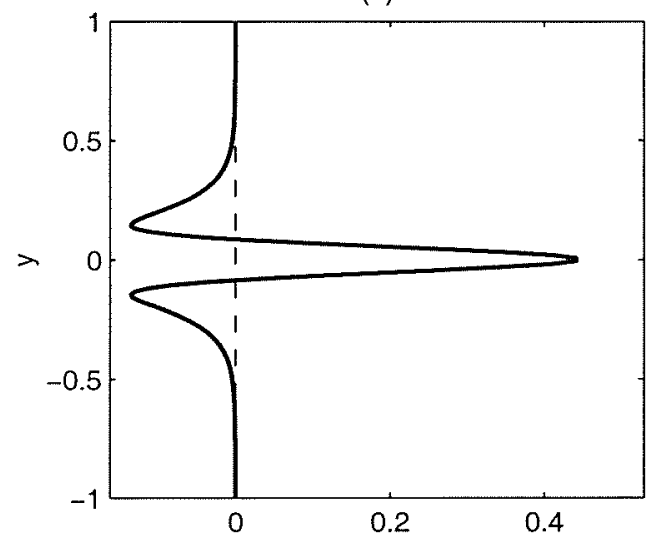

(d)

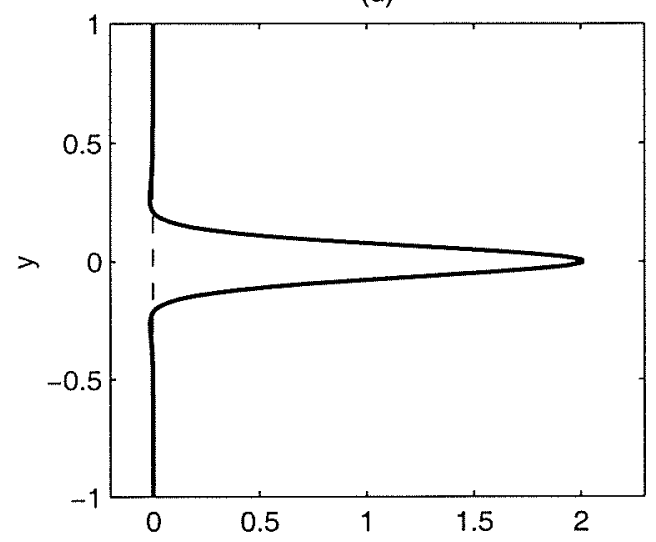

(f)

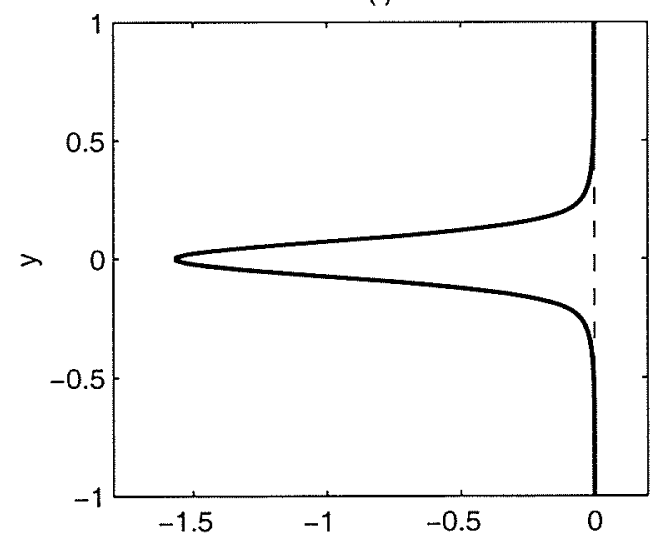

Figure 2-10: (a): Upper layer zonal mean acceleration, $\frac{\partial \bar{u}_{1}}{\partial t}$. (c): Upper layer northward potential vorticity flux, $\overline{v_{1} q_{1}}$. (e): Upper layer residual meridional circulation, $\overline{v_{1}^{*}}$. (b), (d) and (f): same for the lower layer. Parameters: $u_{1}=1, u_{2}=0, \beta=$ $4.15, \gamma=10.18, \delta=0.25, U=0.8 \mathrm{~m} \mathrm{sec}^{-1}$. 
(a)

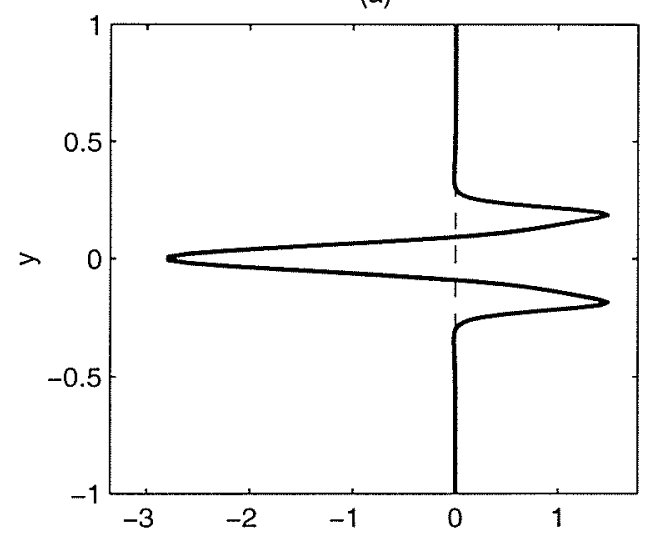

(c)

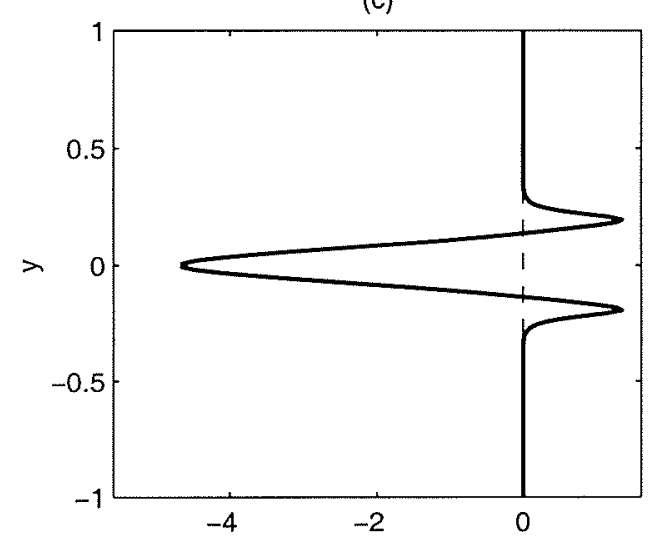

(e)

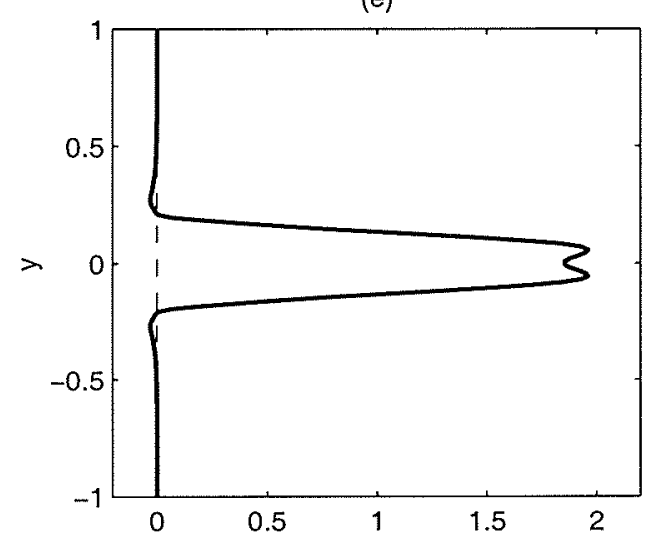

(b)

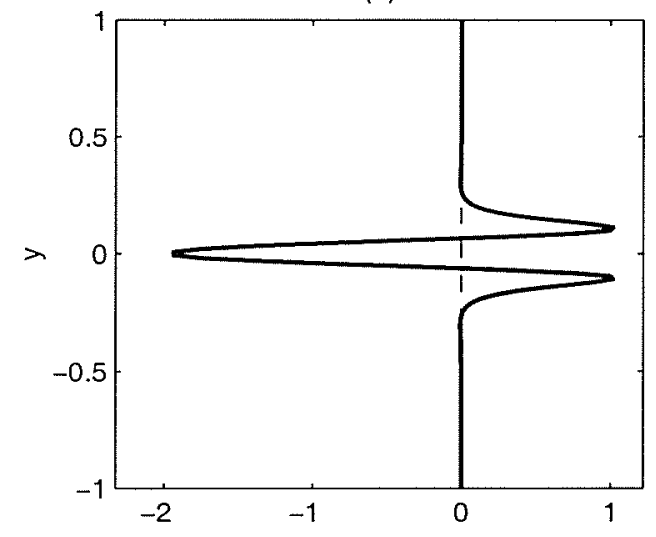

(d)

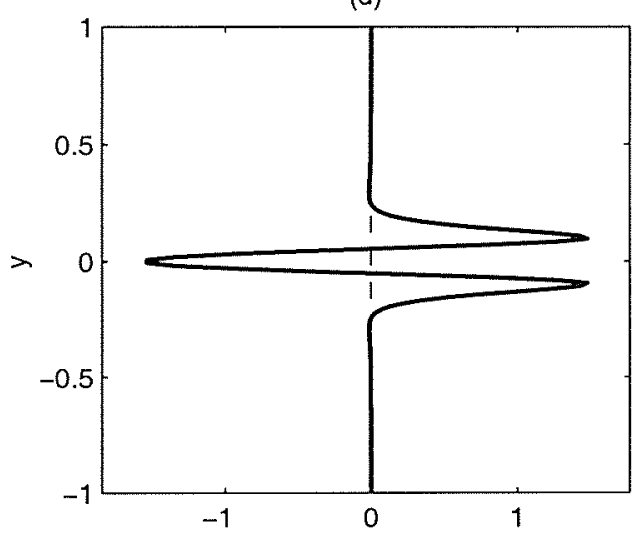

(f)

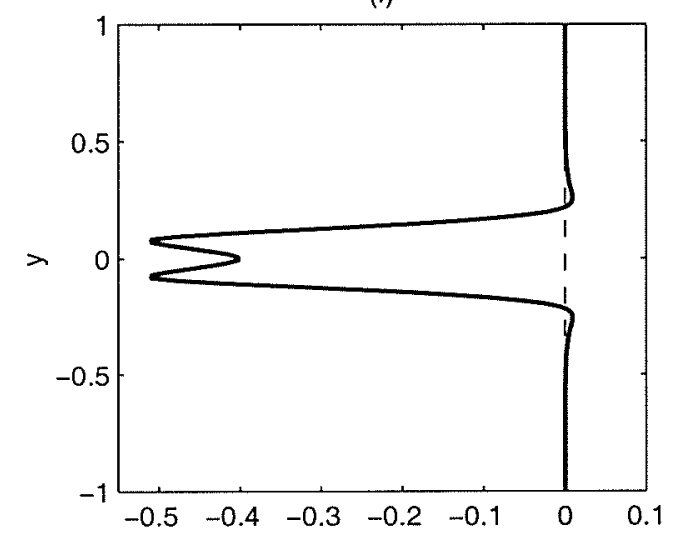

Figure 2-11: (a): Upper layer zonal mean acceleration, $\frac{\partial \bar{u}_{1}}{\partial t}$. (c): Upper layer northward potential vorticity flux, $\overline{v_{1} q_{1}}$. (e): Upper layer residual meridional circulation, $\overline{v_{1}^{*}}$. (b), (d) and (f): same for the lower layer. Parameters: $u_{1}=1, u_{2}=0.2, \beta=$ $4.15, \gamma=10.18, \delta=0.25, U=0.8 \mathrm{~m} \mathrm{sec}^{-1}$. 

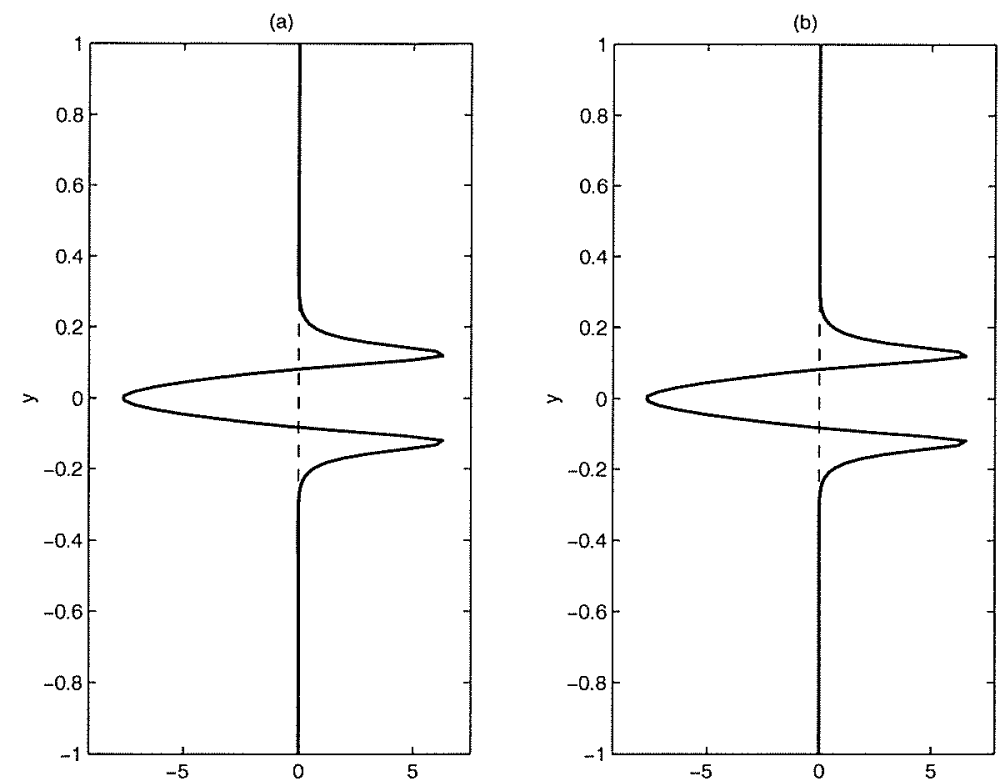

Figure 2-12: (a): Upper layer zonal mean acceleration, $\frac{\partial \bar{u}_{1}}{\partial t}$. (c): Upper layer northward potential vorticity flux, $\overline{v_{1} q_{1}}$. Parameters: $u_{1}=1, u_{2}=0.99, \beta=4.15, \gamma=$ 10.18, $\delta=0.25, U=0.8 \mathrm{~m} \mathrm{sec}^{-1}$.

Figure 2-10a shows mean zonal accelerations, $\frac{\partial \bar{u}_{i}}{\partial t}=-2 k c_{i} \Theta_{i y} e^{2 k c_{i} t}$, for the case when the amplitudes of the upper and lower layer basic velocities are 1.0 and 0.0 respectively. Clearly, the most unstable linear wave has a "diffusive" effect on the upper layer mean flow; it broadens the flow by decreasing its zonal velocity in the middle of the domain and increasing it on the flanks of the jet. In the lower layer, the linearly growing wave tends to induce an eastward jet in the middle of the domain surrounded by weak westward flows on the north and the south. Notice that the amplitude of the lower layer zonal mean acceleration is an order of magnitude smaller than the amplitude in the upper layer.

This contrasts with the mean zonal accelerations for stronger barotropic flows. For both $u_{2}=0.2$ (Figure 2-11a) and $u_{2}=0.99$ (Figure 2-12 a), eddies have purely diffusive effect on the mean flow in both layers. There is no tendency for recirculations in these cases. 
As Equation (2.21) shows, the mean zonal acceleration is defined by the interplay between the residual meridional circulation and the northward eddy potential vorticity fluxes. When $u_{2}=0$, they have opposite impact on the mean flow in the cen-

ter of the jet; $\overline{v^{\prime} q^{\prime}}$ tends to decelerate the mean flow in the upper layer and accelerate it in the lower layer, whereas $v^{*}$ tends to decrease the isopycnal slope, and therefore it is northward (accelerating the mean flow) in the upper layer and southward (decelerating the mean flow) in the lower layer. However, the dominant contribution comes from the eddy potential vorticity fluxes. On the flanks of the jet though, both $\overline{v^{\prime} q^{\prime}}$ and $v^{*}$ tend to induce an eastward flow in the upper layer, so that they both have diffusive effect of the mean flow. In the lower layer, their role is different: it is only due to the residual meridional circulation that there is a tendency for westward flows on the flanks of the jet in the lower layer. As the amplitude of the lower layer nondimensional basic velocity increases, the slope of the isopycnal diminishes. The latter leads to a substantial decrease in the strength of the residual meridional circulation, so that it cannot dominate the eddy potential vorticity fluxes. Therefore, there is no tendency for the formation of recirculations in the lower layer.

For strongly barotropic basic flows, the residual meridional circulation becomes negligible and the eddy potential vorticity fluxes have a purely diffusive effect on the mean flow during the stage of linear growth.

\subsubsection{Relevance to Fully Nonlinear Calculations}

As will be described in section 2.3, the upper layer mean zonal flow decreases its amplitude and becomes broader during the first two months of the nonlinear spin down of an unstable jet. Weak westward recirculations develop in the lower layer. During the next 28 months of integration, the jet sharpens again, increases its amplitude and develops westward recirculations in both layers.

A set of linear stability analysis of broader initial basic flows indicates the tendency for stronger recirculations when the basic flow is strongly baroclinic. Figure 
(a)

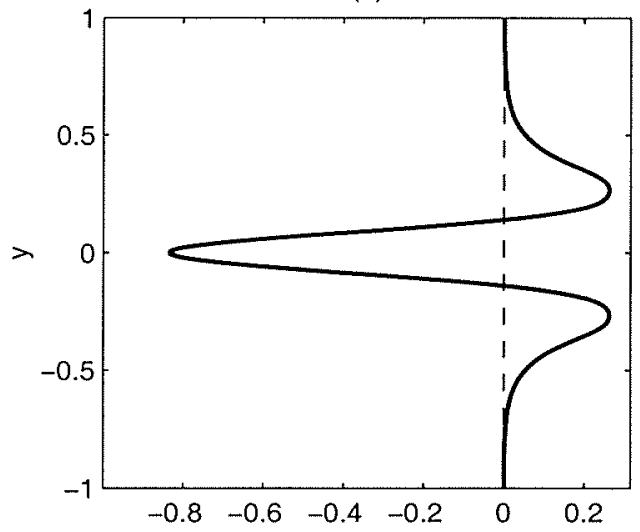

(c)

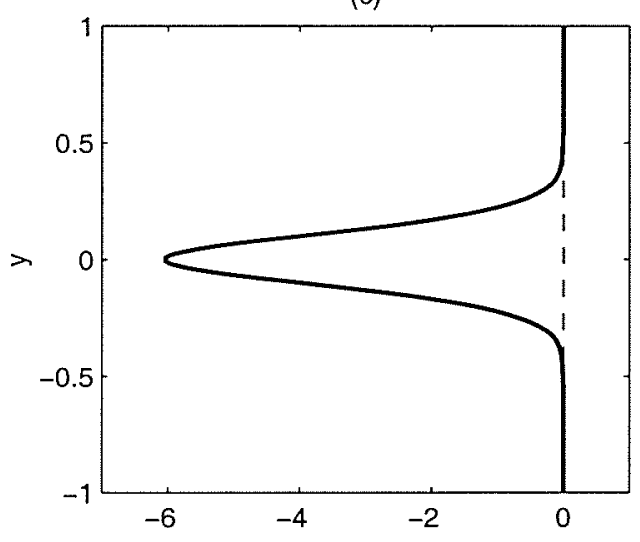

(e)

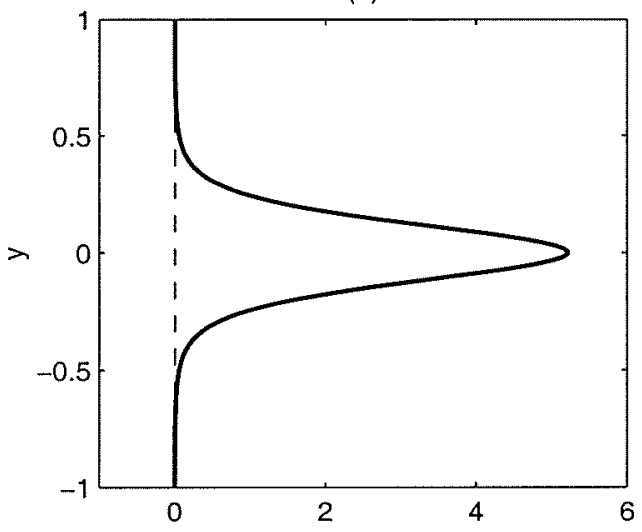

(b)

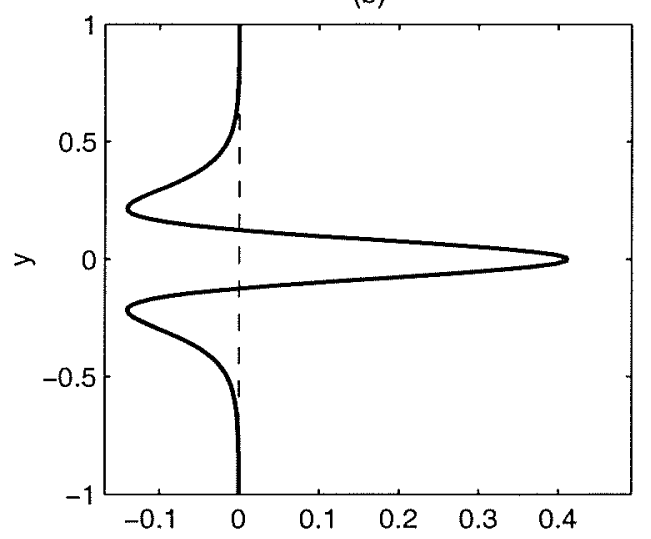

(d)

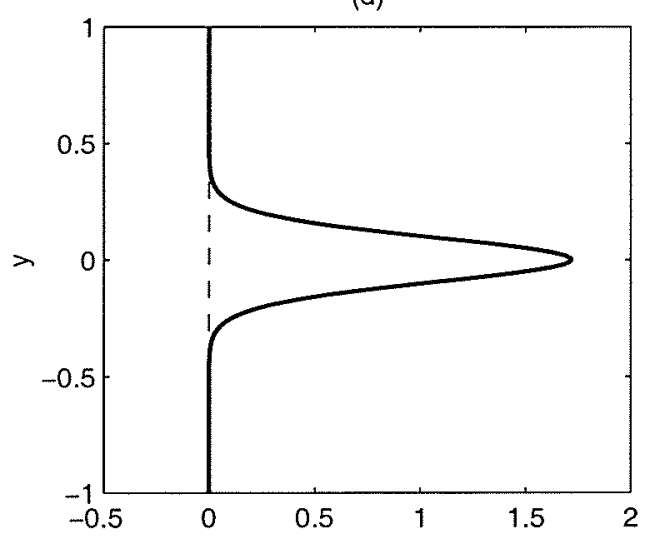

(f)

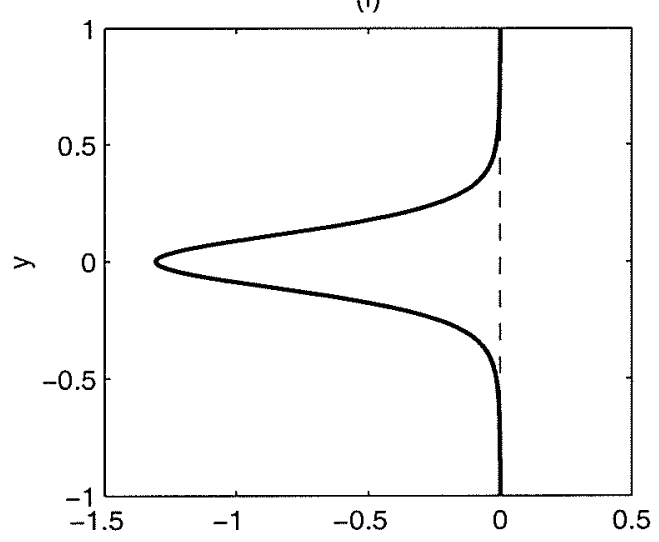

Figure 2-13: (a): Upper layer zonal mean acceleration, $\frac{\partial \bar{u}_{1}}{\partial t}$. (c): Upper layer northward potential vorticity flux, $\overline{v_{1} q_{1}}$. (e): Upper layer residual meridional circulation, $\overline{v_{1}^{*}}$. (b), (d) and (f): same for the lower layer. Parameters: $u_{1}=1, u_{2}=0, \beta=$ $3.32, \gamma=10.18, \delta=0.25, w=5$. 
2-13 shows the results of eddy-mean flow interaction for the jet of $160 \mathrm{~km}$ width. Notice that eddy potential vorticity fluxes act to decelerate the flow in the upper layer and to accelerate the flow in the lower layer. The residual meridional circulation is responsible for diffusing the jet in the upper layer and for inducing the westward retardation in the lower layer. In contrast with a thinner (100 km wide) jet, the amplitudes of mean zonal accelerations are of the same order of magnitude in both layers. In strongly barotropic flows, the tendency for westward recirculations is absent. As we increase the width of the jet, the flow stabilizes.

To see if the linear mean-flow interaction alone could be responsible for the formation of the recirculations we used an eddy-mean flow interaction model, developed by Flierl (personal communication). The model equations are:

$$
\begin{gathered}
\frac{\partial \bar{u}_{i}}{\partial t}=(-1)^{i} F_{i} \chi+\overline{v_{i}^{\prime} q_{i}^{\prime}} \\
\frac{\partial q_{i}^{\prime}}{\partial t}+\bar{u}_{i} \frac{\partial q_{i}^{\prime}}{\partial x}+v_{i}^{\prime} \frac{\partial \bar{q}_{i}}{\partial y}=0 \\
\left(\frac{\partial^{2}}{\partial y^{2}}-F_{1}-F_{2}\right) \chi=\overline{v_{1}^{\prime} q_{1}^{\prime}}-\overline{v_{2}^{\prime} q_{2}^{\prime}}
\end{gathered}
$$

where $\bar{u}$ and $\bar{q}$ are mean zonal geostrophic velocity vector and mean potential vorticity, $\left(u^{\prime}, v^{\prime}\right)^{T}$ and $q^{\prime}$ are perturbation geostrophic velocity and perturbation potential vorticity, $\chi$ is residual meridional circulation and $i=1,2$ is layer index.

The model was applied for a basic flow of the form $\bar{u}_{i}(y)=A_{i} \cosh ^{2}(y)$ and converged only for strongly baroclinic initial basic flows; the procedure was divergent for strongly barotropic flows. The resulting basic flow had well developed westward flows. Thus, eddy-mean flow interactions were sufficient to explain the development of recirculations in strongly baroclinic cases.

This approach does not work for barotropic flows, since strongly nonlinear regimes allowing for both eddy-mean flow and eddy-eddy interactions are required for recirculation development. 


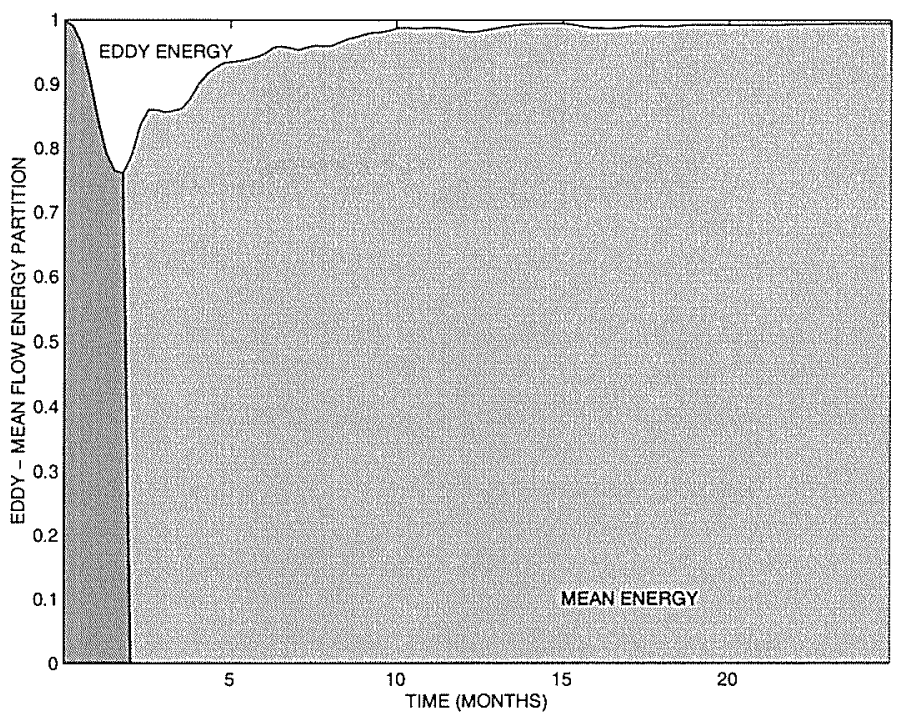

Figure 2-14: Eddy - mean flow partition of total energy vs dimensional time. The dark-shaded area indicates the mean flow energy part, the white area indicates the eddy energy part. Parameters: $u_{1}=1, u_{2}=0, \beta=4.15, \gamma=10.18, \delta=0.25, U=$ $0.8 \mathrm{~m} \mathrm{sec}^{-1}$.

\subsection{Nonlinear Analysis of a Two-Layer Model}

\subsubsection{The Flow Evolution}

In all the numerical experiments, flow evolution goes through two separate stages. During the first stage, the eddy energy grows linearly at the expense of the mean flow energy (Figure 2-14). In the streamfunction field (Figure 2-15), this stage corresponds to the development of instabilities, to the growth of meanders and, in strongly barotropic cases, to ring formation. The second stage begins when the eddy energy reaches its maximum. Eddies, radiated away from the jet, start releasing energy back to the mean flow thus restructuring it. The equilibrated flow has a wider and weaker eastward jet surrounded by westward flows to the north and south.

Total integration time of the model is equivalent to 30 months. Total net transport is constant over time (Figure 2-16). Both integrated eastward and westward 
(a)

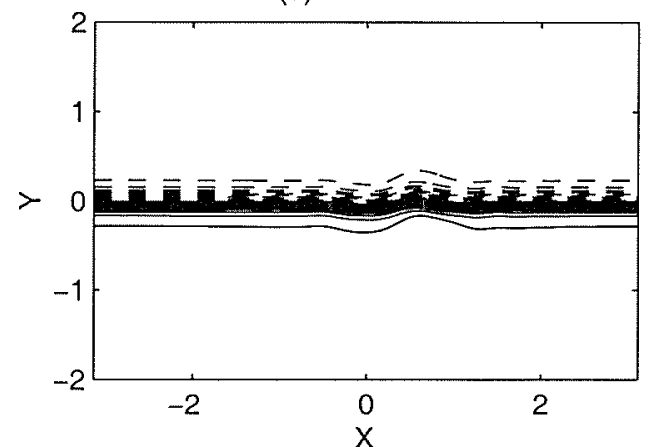

(b)

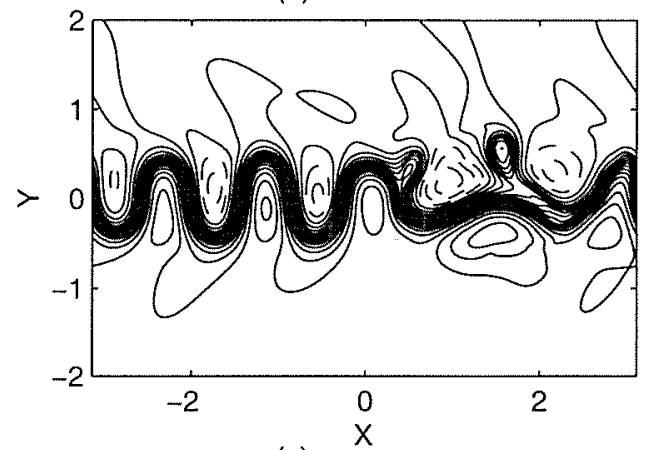

(c)

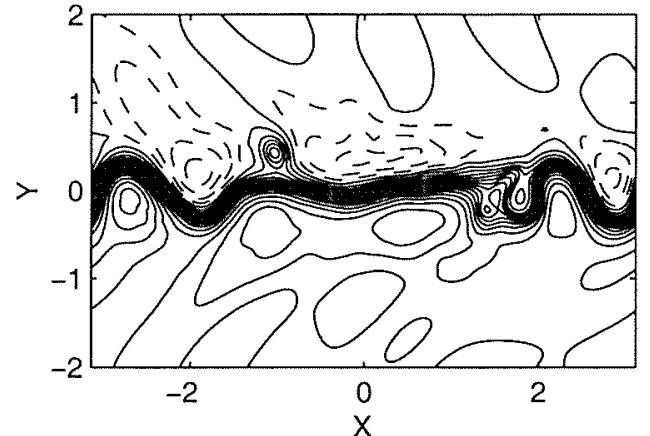

(d)

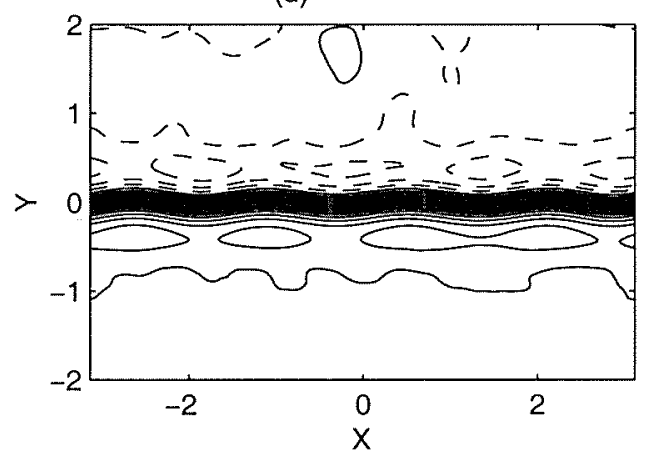

(e)

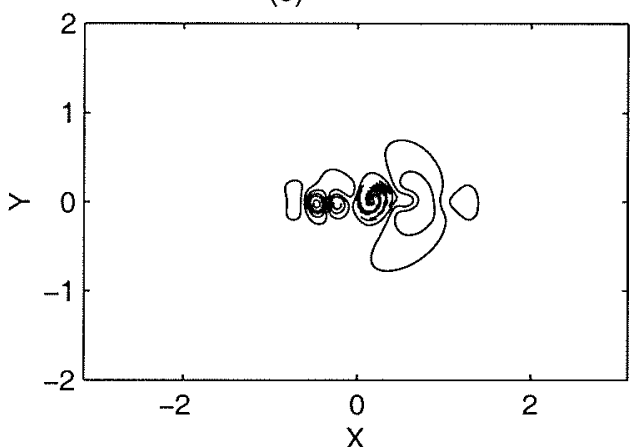

(f)

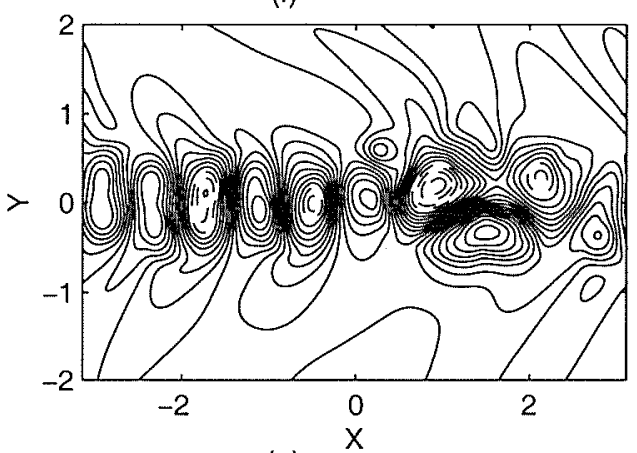

(g)

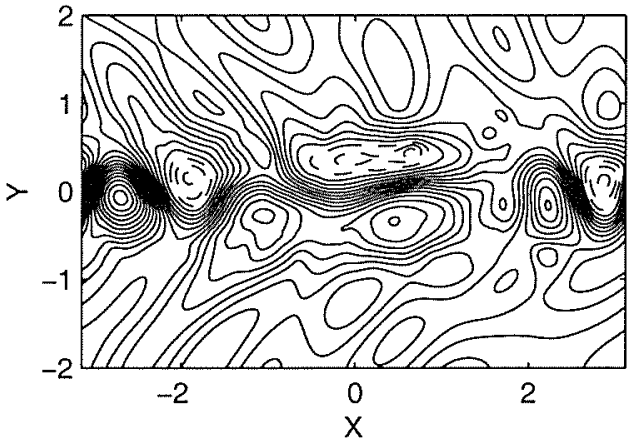

(h)

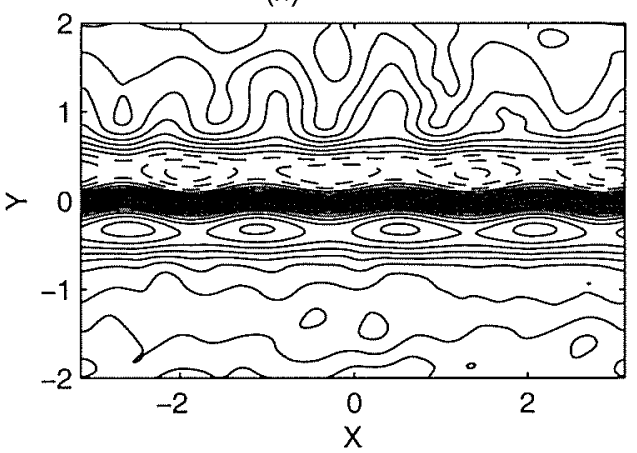

Figure 2-15: Contours of instantaneous upper layer stream function after 10 days (a), 66 days (b), 4.5 months (c) and 30 months (d). Same for the lower layer: (e)-(f). Parameters: $u_{1}=1, u_{2}=0, \beta=4.15, \gamma=10.18, \delta=0.25, U=0.8 \mathrm{~m} \mathrm{sec}^{-1}$. 


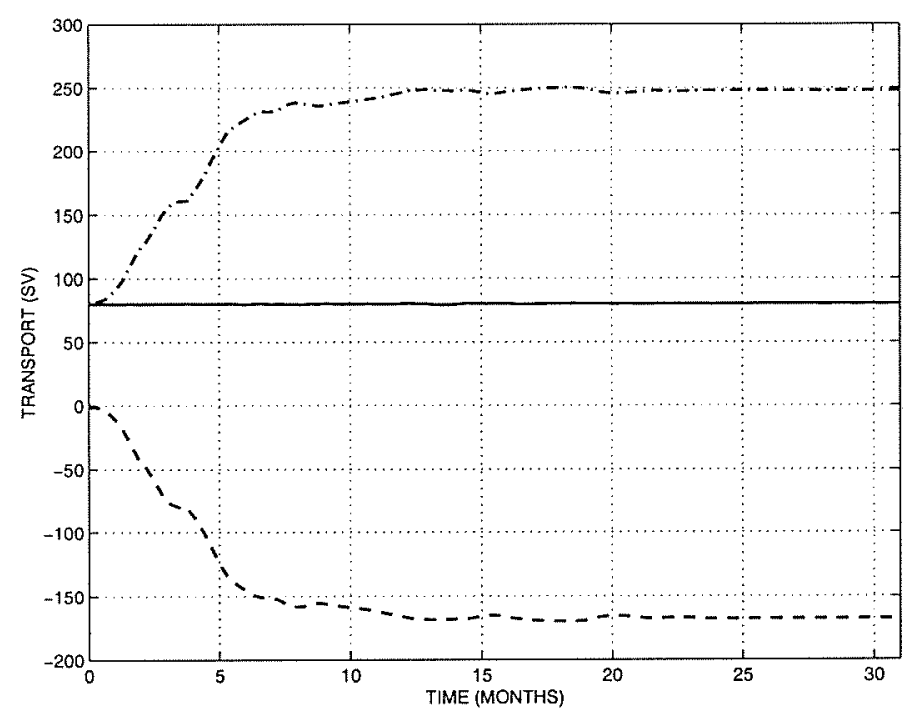

Figure 2-16: Dimensional total net (solid), eastward (dash-dot) and westward (dash) transports as functions of dimensional time. Parameters: $u_{1}=1, u_{2}=0, \beta=$ $4.15, \gamma=10.18, \delta=0.25, U=0.8 \mathrm{~m} \mathrm{sec}^{-1}$.

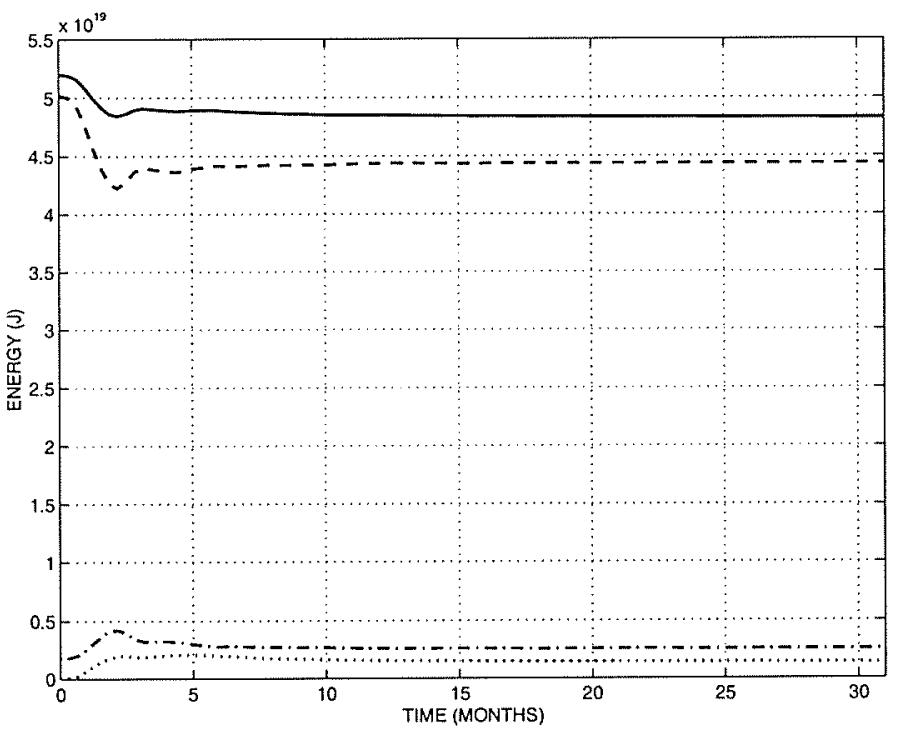

Figure 2-17: Total (solid), potential (dash), upper layer kinetic (dash-dot) and lower layer kinetic (dot) energy. Parameters: $u_{1}=1, u_{2}=0, \beta=4.15, \gamma=10.18, \delta=$ $0.25, U=0.8 \mathrm{~m} \mathrm{sec}^{-1}$. 


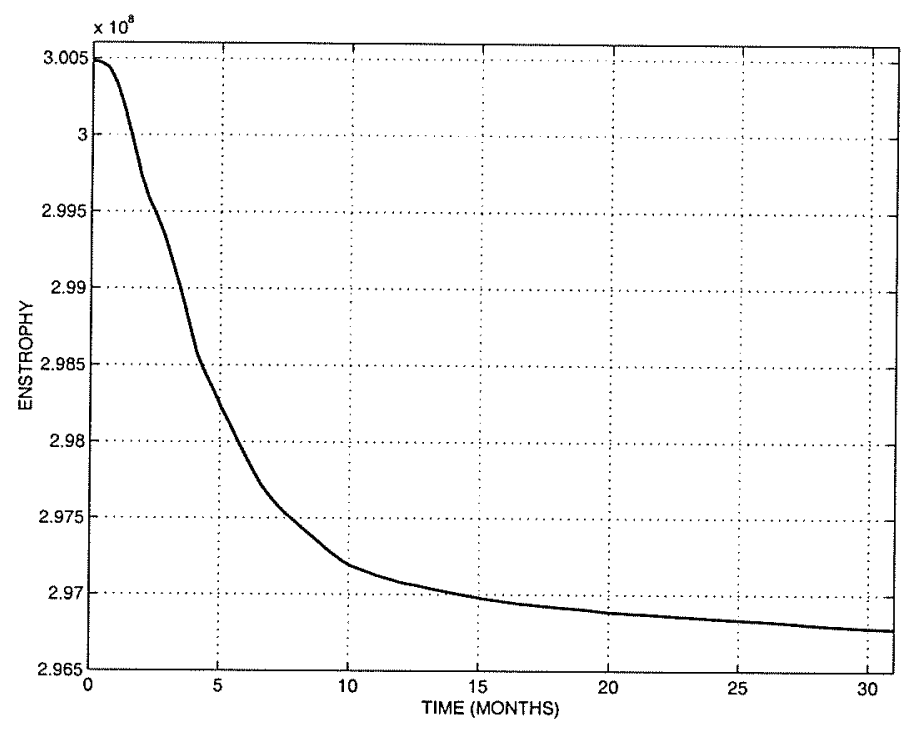

Figure 2-18: Total enstrophy vs dimensional time. Parameters: $u_{1}=1, u_{2}=0, \beta=$ $4.15, \gamma=10.18, \delta=0.25, U=0.8 \mathrm{~m} \mathrm{sec}^{-1}$.

transports increase linearly for the first 7 months, saturating completely after 15 months.

Since the model has two sponge layers and a weak numerical filter, total energy slowly decreases with time (Figure 2-17). The maximum decay corresponds to the first 5 months of model integration, when intensely growing eddies radiate away from the jet and enter the sponge layers. Once eddies start releasing energy back to the mean flow, the energy decay diminishes substantially.

Time fluctuations of total momentum are less than $0.005 \%$ of the total initial field. Total enstrophy decreases with time by about $5 \%$ (Figure 2-18), with maximum decay during the stage of eddy energy growth.

\subsubsection{The Equilibrated Jet Structure}

This sections discusses the differences in the equilibrated jet structures for baroclinic and barotropic initial basic flows. The initial basic velocity is chosen such that $u_{1}=1$ 
(a)

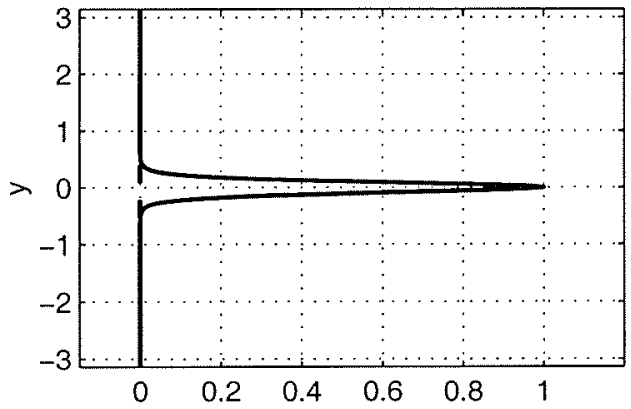

(b)

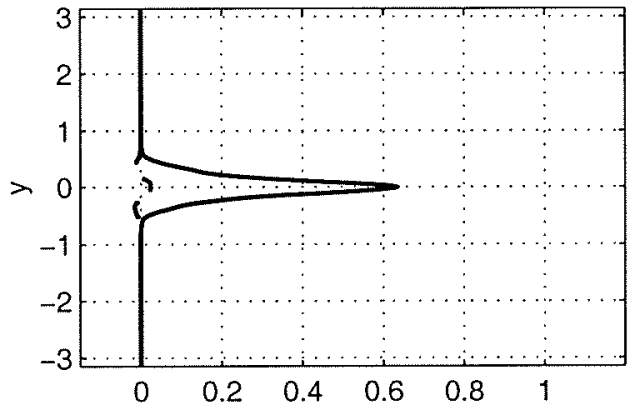

(c)

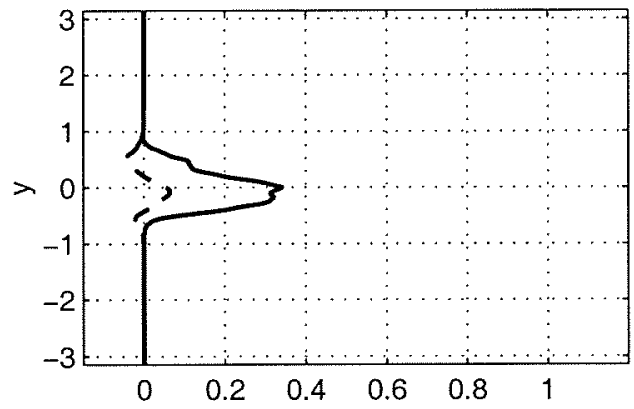

(d)

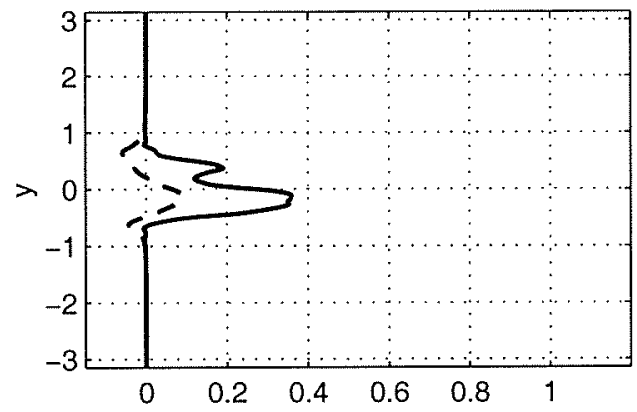

(e)

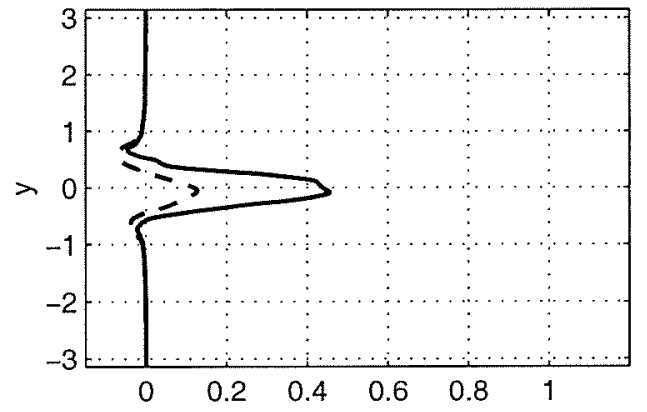

(f)

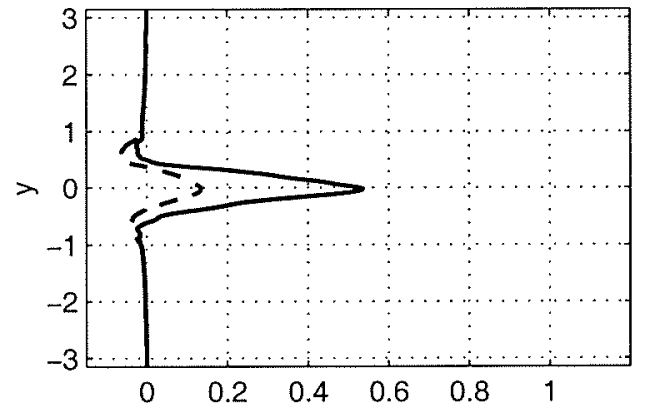

(g)

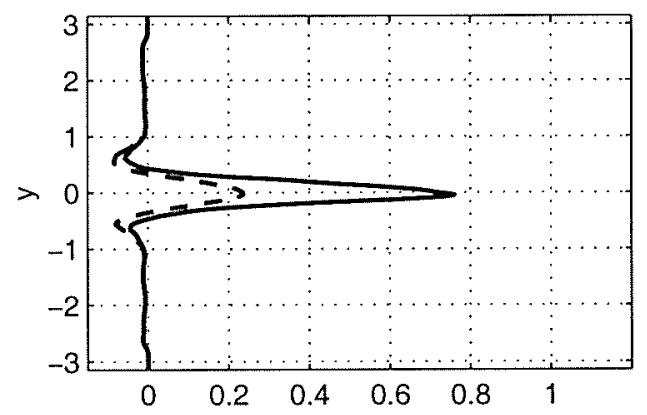

(h)

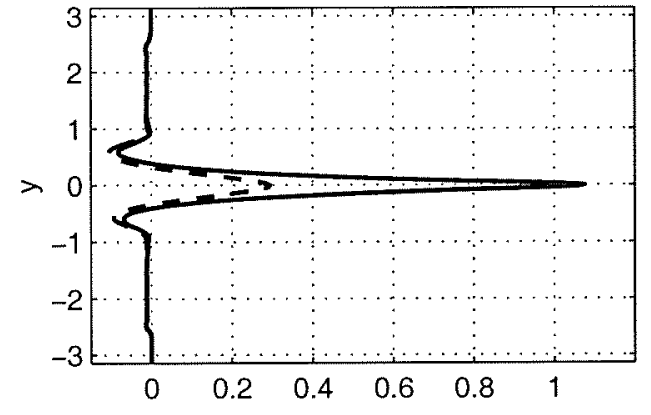

Figure 2-19: Upper (solid) and lower (dash) layer mean zonal velocity, $\overline{U_{i}}(y)=$ $\frac{1}{2 \pi} \int_{-\pi}^{\pi} U_{i}(x, y) d x, i=1,2$, at $\mathrm{t}=0$ (a), 28 days (b), 47 days (c), 66 days (d), 3 months (e), 4 months (f), 6 months (g), 30 months (h). Parameters: $u_{1}=1, u_{2}=0, \beta=$ $4.15, \gamma=10.18, \delta=0.25, U=0.8 \mathrm{~m} \mathrm{sec}^{-1}$. 
and $u_{2}$ varies between zero and 0.99 . In all the experiments, the initially unstable jet stabilizes, and its structure changes drastically with time.

Figure 2-19 shows the time evolution of the mean zonal velocity for the case when initially there is no flow in the lower layer (a). In the first month (b), when eddies grow at the expense of the mean flow energy, the upper layer jet decreases its amplitude and slightly widens. In the lower layer, a weak eastward flow develops in the middle of the domain surrounded by two weak westward flows. Between 1.5 (c) and 2 months (d) of flow evolution, this lower layer structure strengthens, while the upper layer mean zonal velocity loses its well defined jet profile due to the still growing eddy field. After 6 months (g), eddies pump energy back to the mean flow, and the equilibrated flow (h) consists of a sharp baroclinic jet (with a nonzero amplitude in the lower layer) surrounded by barotropic westward flows on the north and south. Figure 2-20 shows the mean flow evolution for strongly barotropic flows. The process is similar for strongly barotropic basic flows (Figure 2-20).

As the nondimensional lower layer velocity amplitude, $u_{2}$, varies from zero to 0.99 , the final maximum of the mean dimensional zonal velocity decreases from 0.88 to $0.53 \mathrm{~m} \mathrm{sec}^{-1}$ in the upper layer and increases from 0.23 to $0.53 \mathrm{~m} \mathrm{sec}^{-1}$ in the lower layer (Figure 2-21a). Interestingly, when $u_{2}=0$, the maximum final upper layer velocity is larger than the maximum initial upper layer velocity. The dimensional width of the upper layer jet increases monotonically from 180 to $220 \mathrm{~km}$. In the lower layer, it diminishes from 140 to $120 \mathrm{~km}$ as $u_{2}$ increases from 0.0 to 0.5 , and then grows to $210 \mathrm{~km}$ for $u_{2}>0.5$.

\subsubsection{Potential Vorticity Mixing}

The equilibration of an unstable jet can be viewed through the process of potential vorticity mixing that acts to remove a negative potential vorticity gradients. Such a process differs for baroclinic versus barotropic jets. In the following discussion, we 
(a)

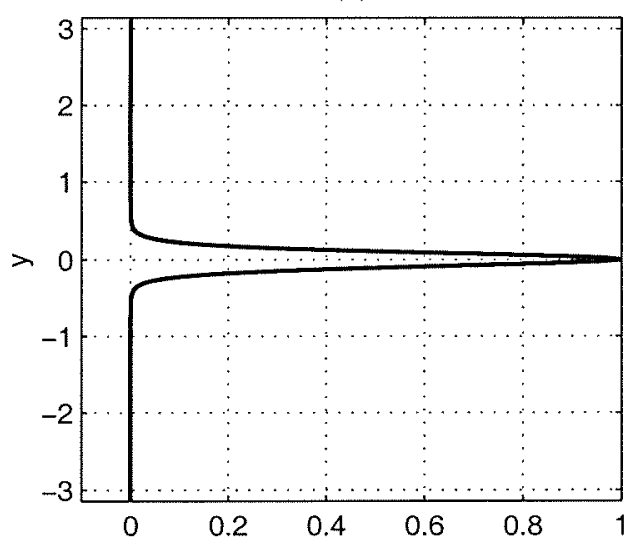

(b)

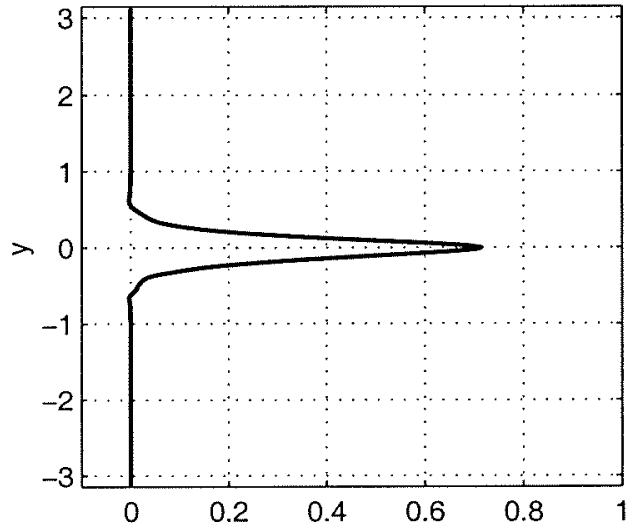

(c)

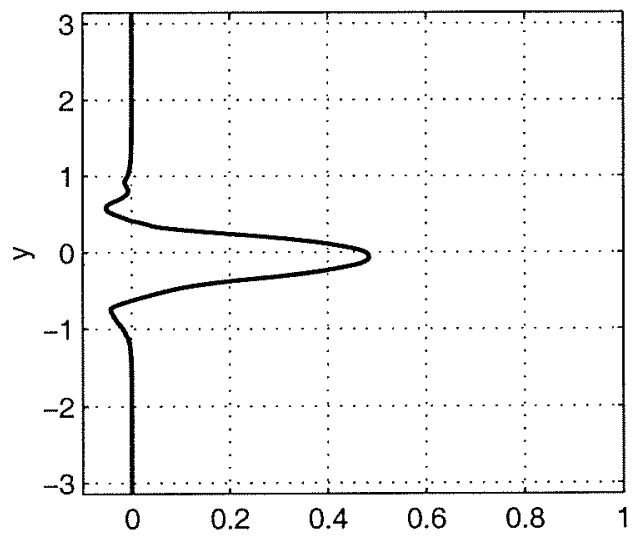

(d)

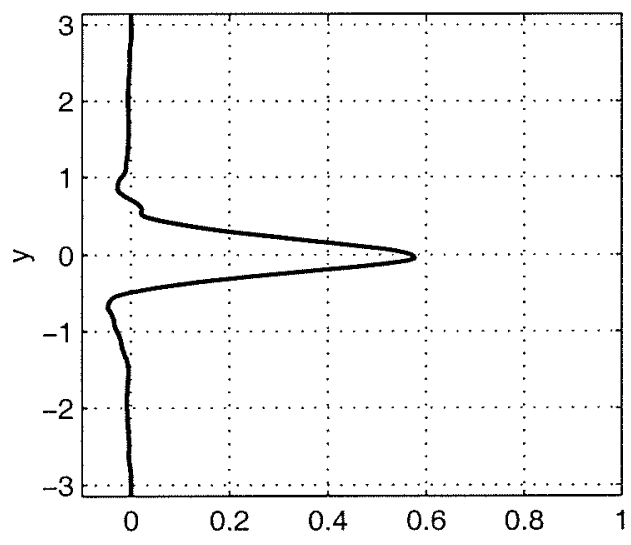

(e)

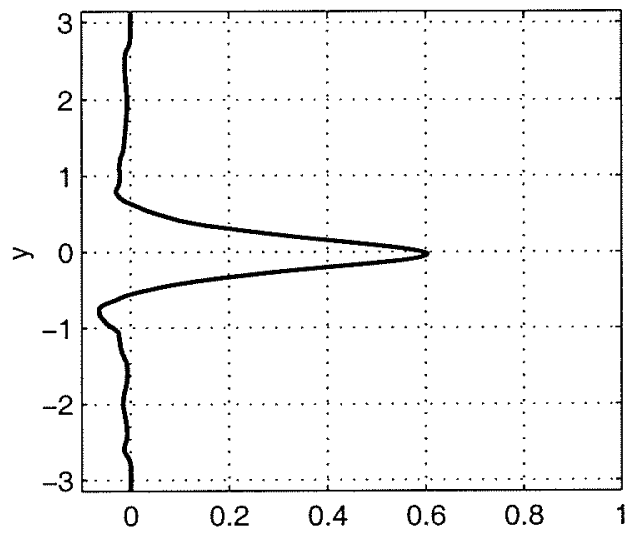

(f)

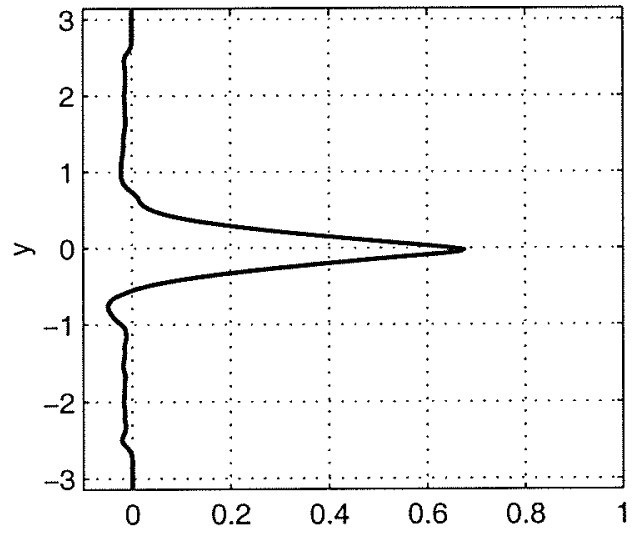

Figure 2-20: Upper layer mean zonal velocity, $\overline{U_{1}}(y)=\frac{1}{2 \pi} \int_{-\pi}^{\pi} U_{1}(x, y) d x$, at $\mathrm{t}=0$ (a), 18 days (b), 47 days (c), 66 days (d), 9 months (e), 30 months (f). Parameters: $u_{1}=1, u_{2}=0.99, \beta=4.15, \gamma=10.18, \delta=0.25, U=0.8 \mathrm{~m} \mathrm{sec}^{-1}$. 

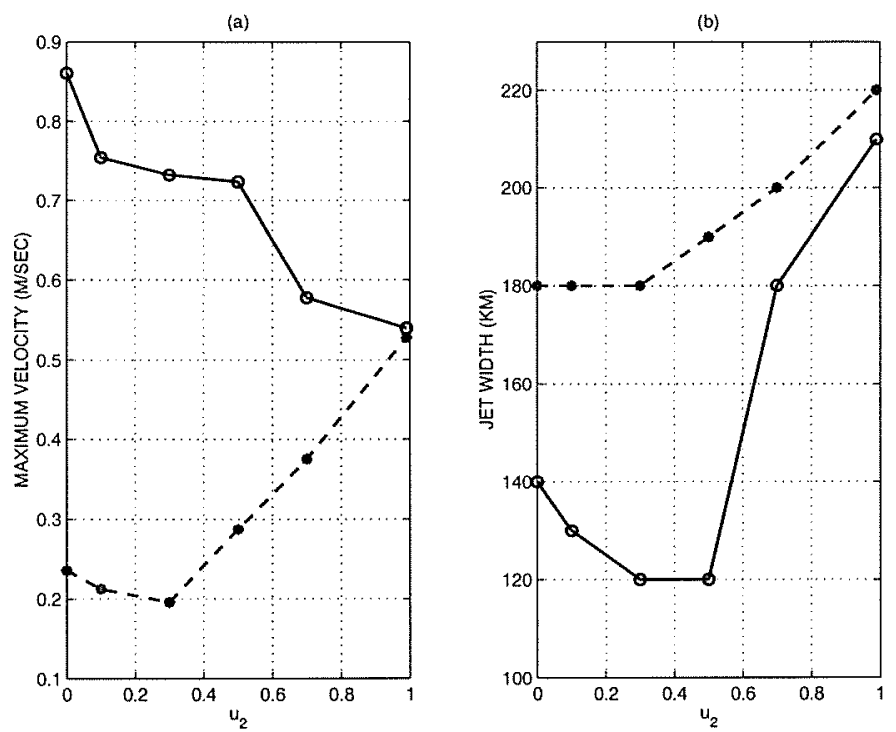

Figure 2-21: (a): Maximum of dimensional velocity, $U u_{i}$, ( in $\left.\mathrm{m} \mathrm{sec}^{-1}\right)$ of the equilibrated jet in the upper (solid) and lower (dash) layers versus the nondimensional amplitude of the initial lower layer velocity, $u_{2}$. Parameters: $u_{1}=1, \beta=4.15, \gamma=$ 10.18, $\delta=0.25, U=0.8 \mathrm{~m} \mathrm{sec}^{-1}$. (b): Dimensional width (in $\mathrm{km}$ ) of the equilibrated jet in the upper (solid) and lower (dash) layer versus the nondimensional amplitude of the initial lower layer velocity, $u_{2}$.

contrast two examples: $\left(u_{1}, u_{2}\right)=(1,0)$ which corresponds to a strongly baroclinic jet, and $\left(u_{1}, u_{2}\right)=(1.0,0,99)$ which corresponds to a strongly barotropic jet.

For $u_{2}=0$, both the upper and the lower layer potential vorticity fields have regions of negative gradient (Figure 2-22a). After equilibration, the lower layer potential vorticity is homogenized in a wide zone under the axis of the jet (Figure 2-22b). Hence, the flow is only marginally stable there. Zero $q$-gradient indicates that there is intense mixing in the lower layer in the center of the domain. Similar homogenization of lower layer potential vorticity was observed in a two-layer $\beta$-plane model of Pedlosky [28], where he studied weakly nonlinear equilibration of Phillips' model with weak dissipation.

In the rest of the lower layer domain, there is a weak positive potential vorticity gradient which corresponds to planetary $\beta$. In the upper layer, the region of the eastward jet with large and positive $q$-gradient is surrounded on the north and 

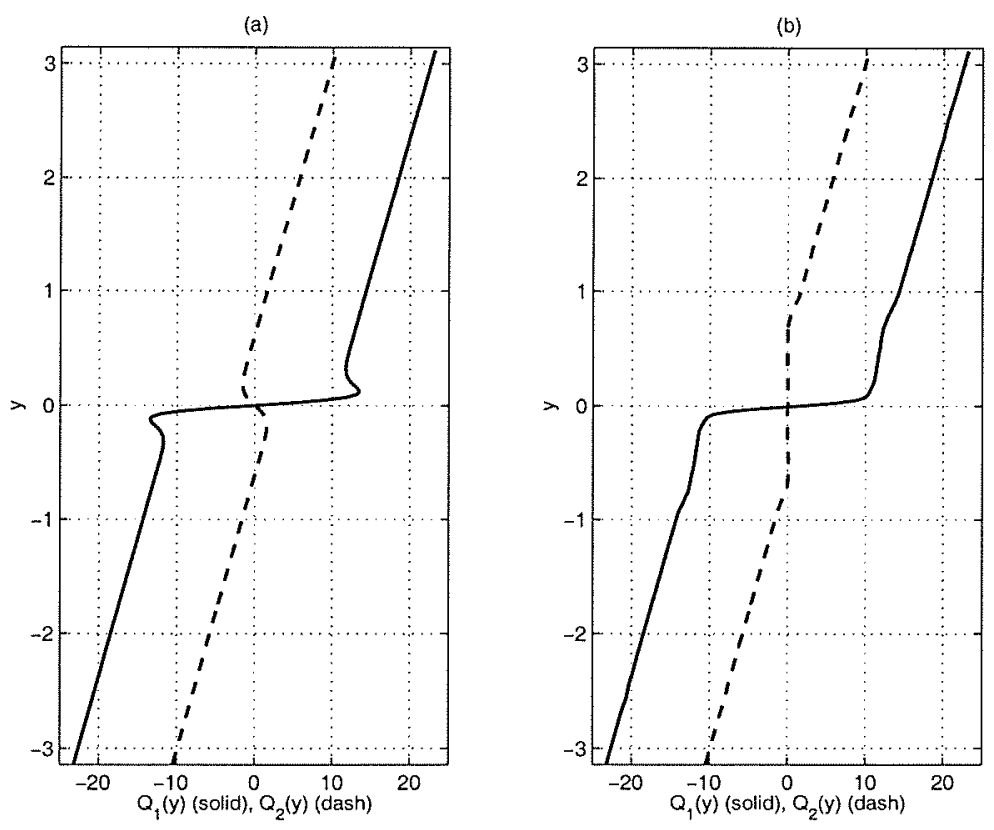

Figure 2-22: Initial (a) and final (b) mean zonal potential vorticity, $\overline{q_{i}}(y)=$ $\frac{1}{2 \pi} \int_{-\pi}^{\pi} q_{i}(x, y) d x, i=1,2$, for the upper (solid) and lower (dash) layer. Parameters: $u_{1}=1, u_{2}=0, \beta=4.15, \gamma=10.18, \delta=0.25, U=0.8 \mathrm{~m} \mathrm{sec}^{-1}$.
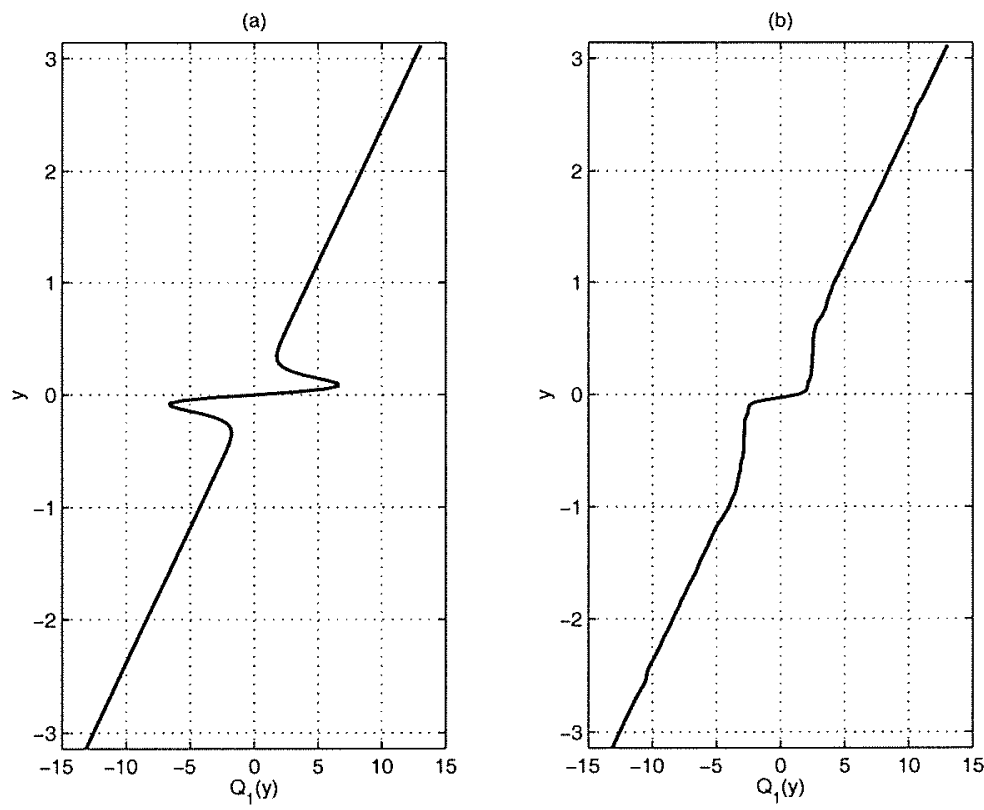

Figure 2-23: Initial (a) and final (b) mean zonal potential vorticity for the upper layer, $\overline{q_{1}}(y)=\frac{1}{2 \pi} \int_{-\pi}^{\pi} q_{1}(x, y) d x$. Parameters: $u_{1}=1, u_{2}=0.99, \beta=4.15, \gamma=10.18, \delta=$ $0.25, U \stackrel{2 \pi}{=} 0.8 \mathrm{~m} \mathrm{sec}^{-1}$. 
(a)

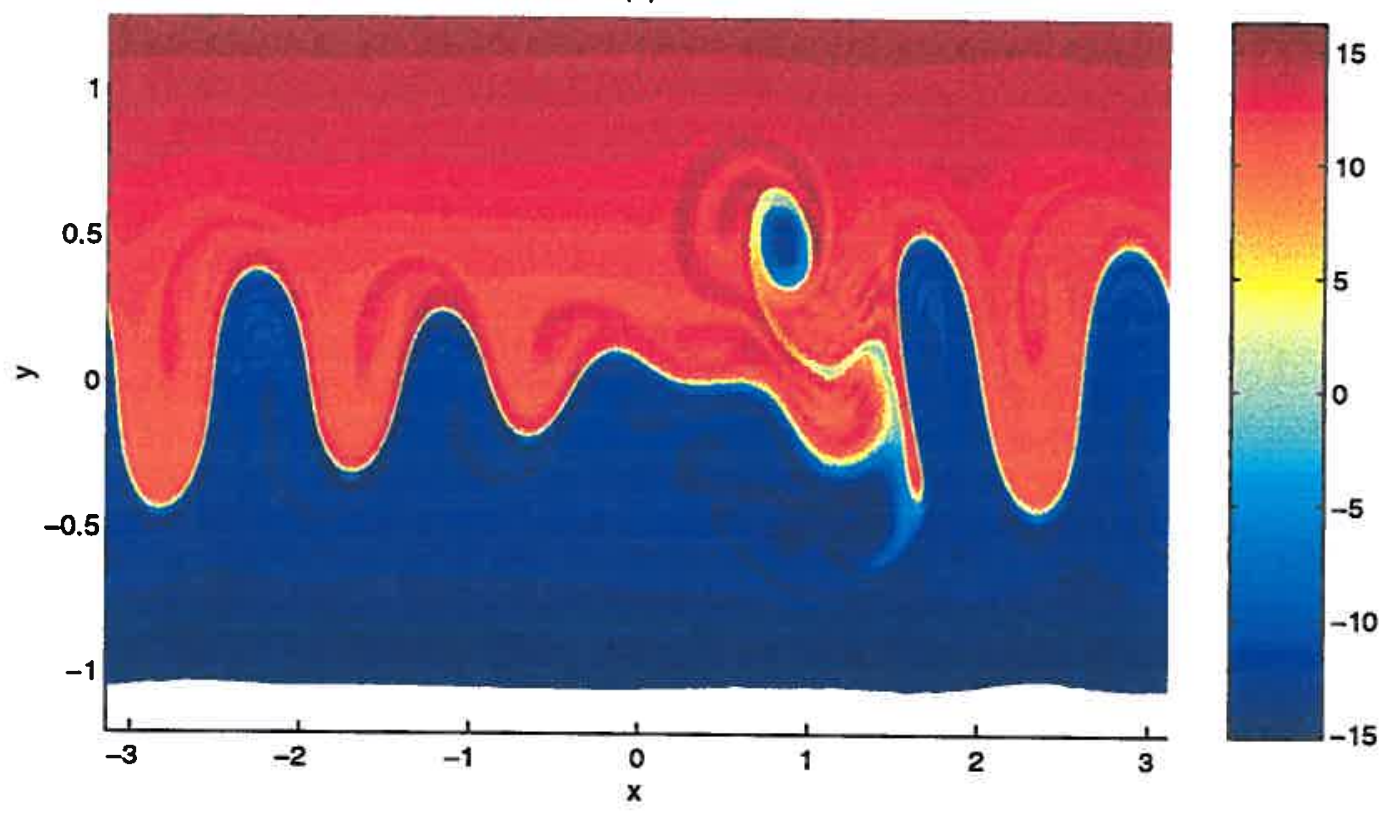

(b)

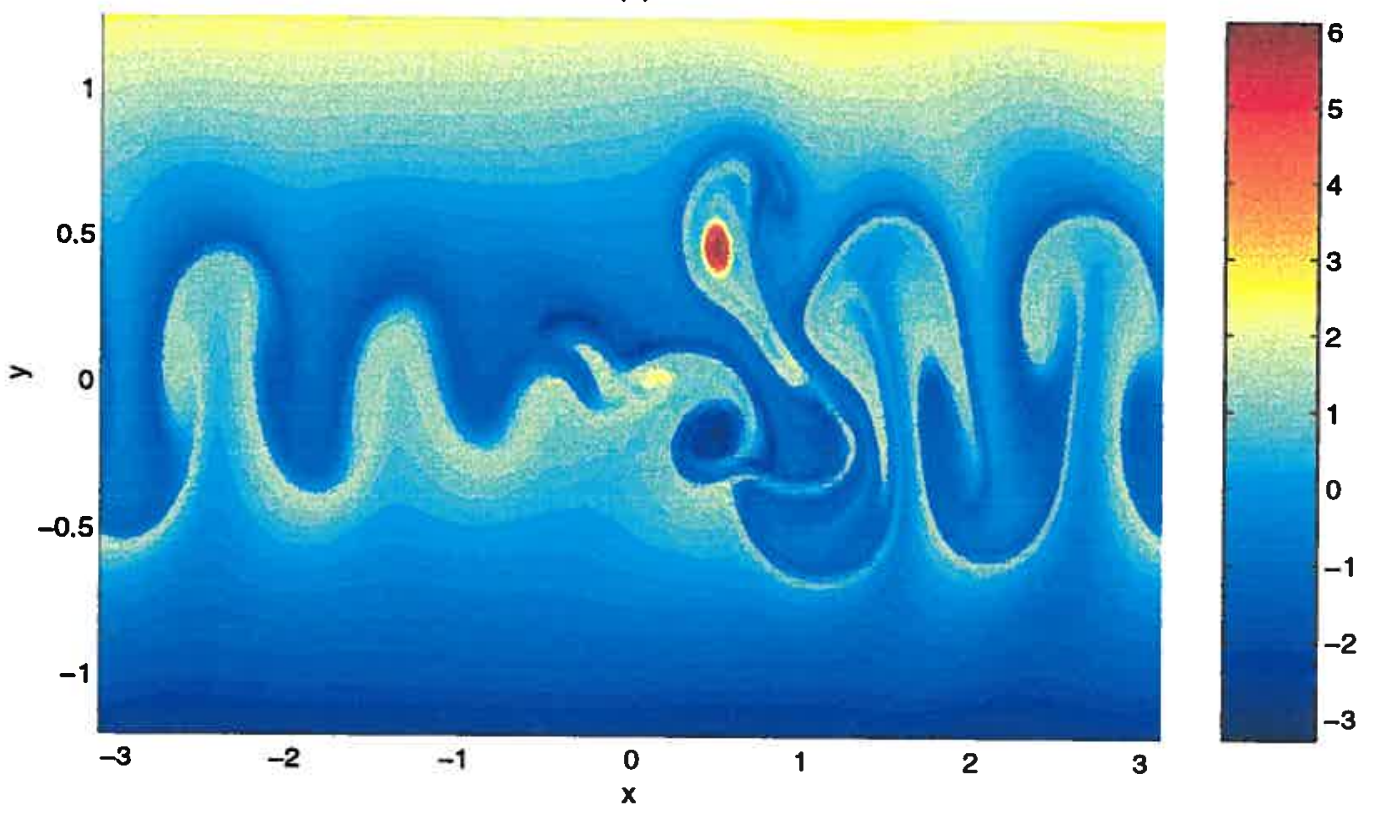

Figure 2-24: (a): Contours of the upper layer potential vorticity at $t=47$ days. (b): Same for the lower layer. Parameters: $u_{1}=1, u_{2}=0, \beta=4.15, \gamma=10.18, \delta=$ $0.25, U=0.8 \mathrm{~m} \mathrm{sec}^{-1}$. 
(a)

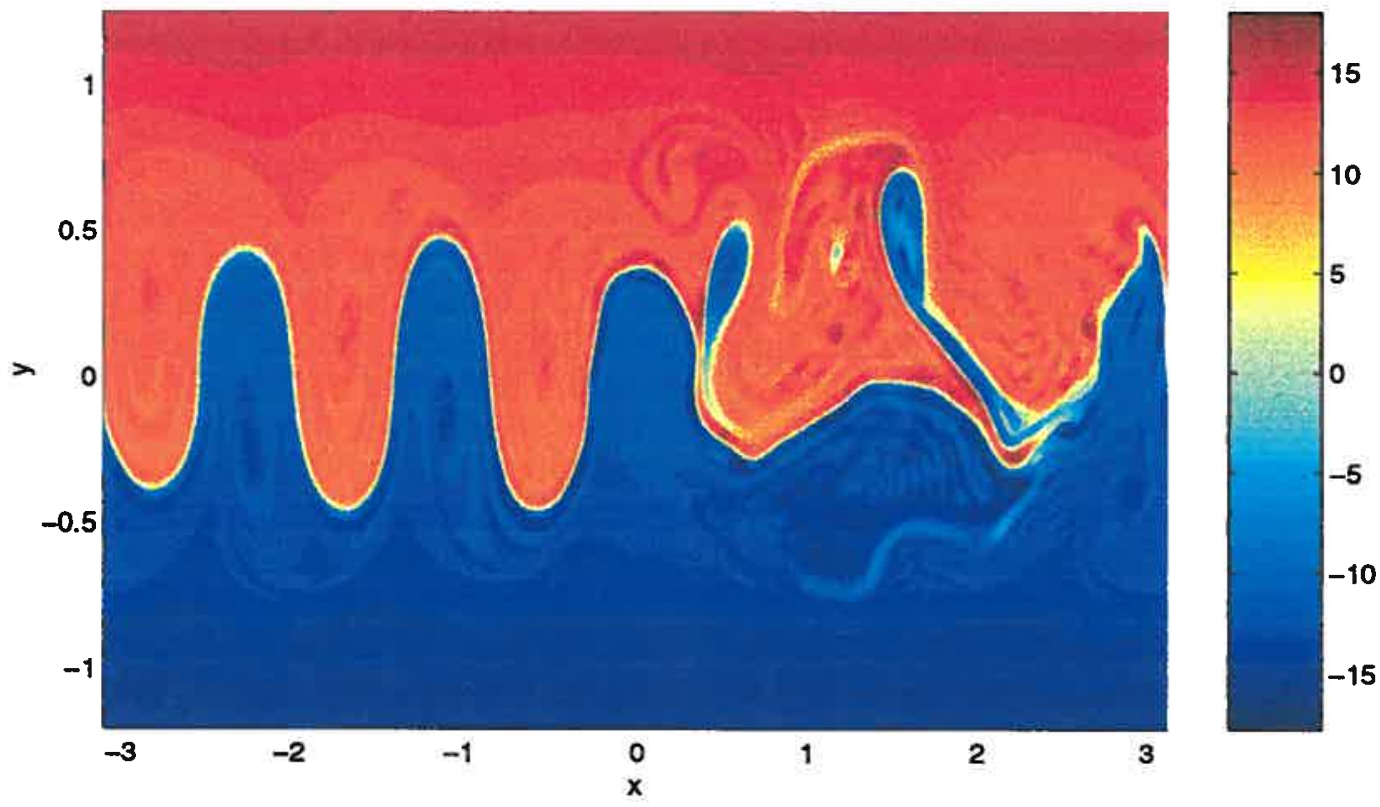

(b)

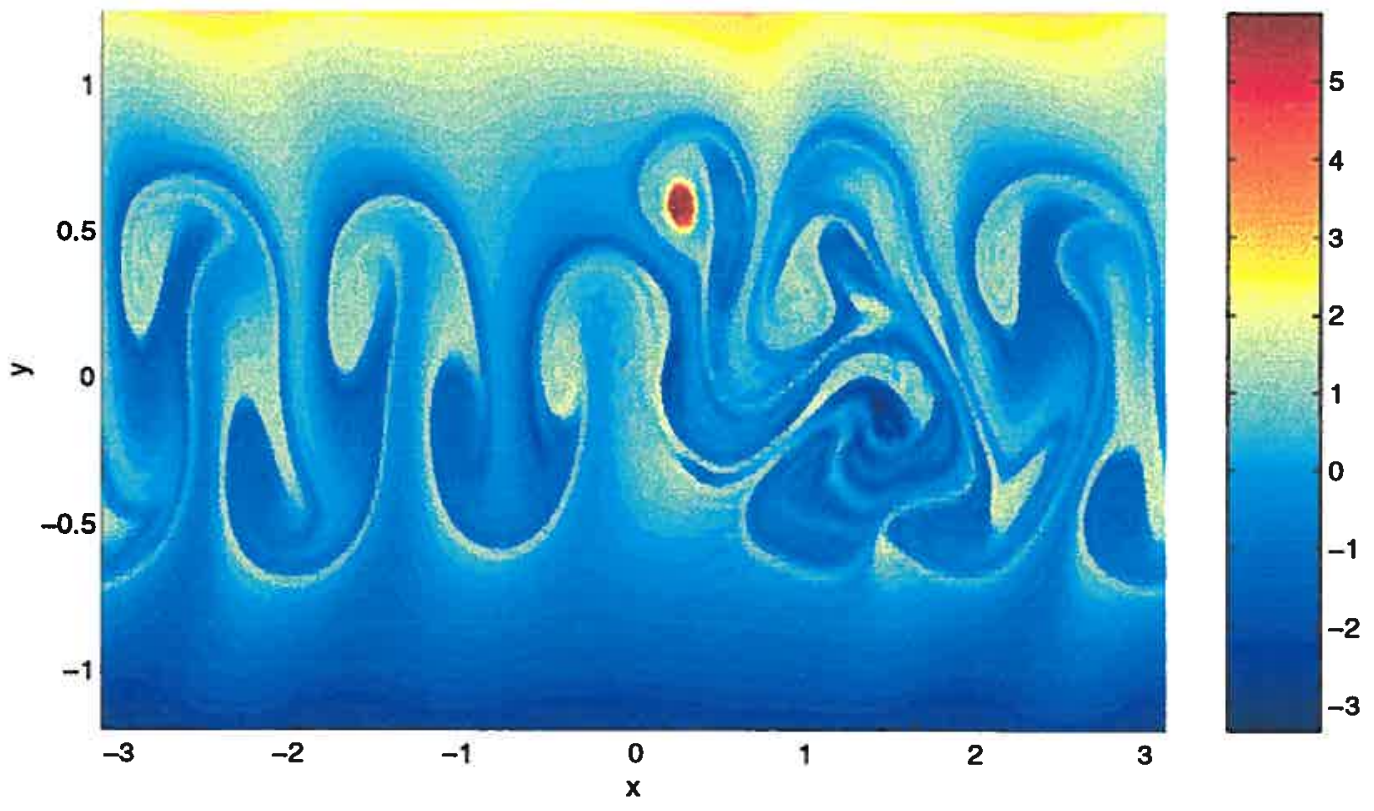

Figure 2-25: (a): Contours of the upper layer potential vorticity at $t=66$ days. (b): Same for the lower layer. Parameters: $u_{1}=1, u_{2}=0, \beta=4.15, \gamma=10.18, \delta=$ $0.25, U=0.8 \mathrm{~m} \mathrm{sec}^{-1}$. 
(a)

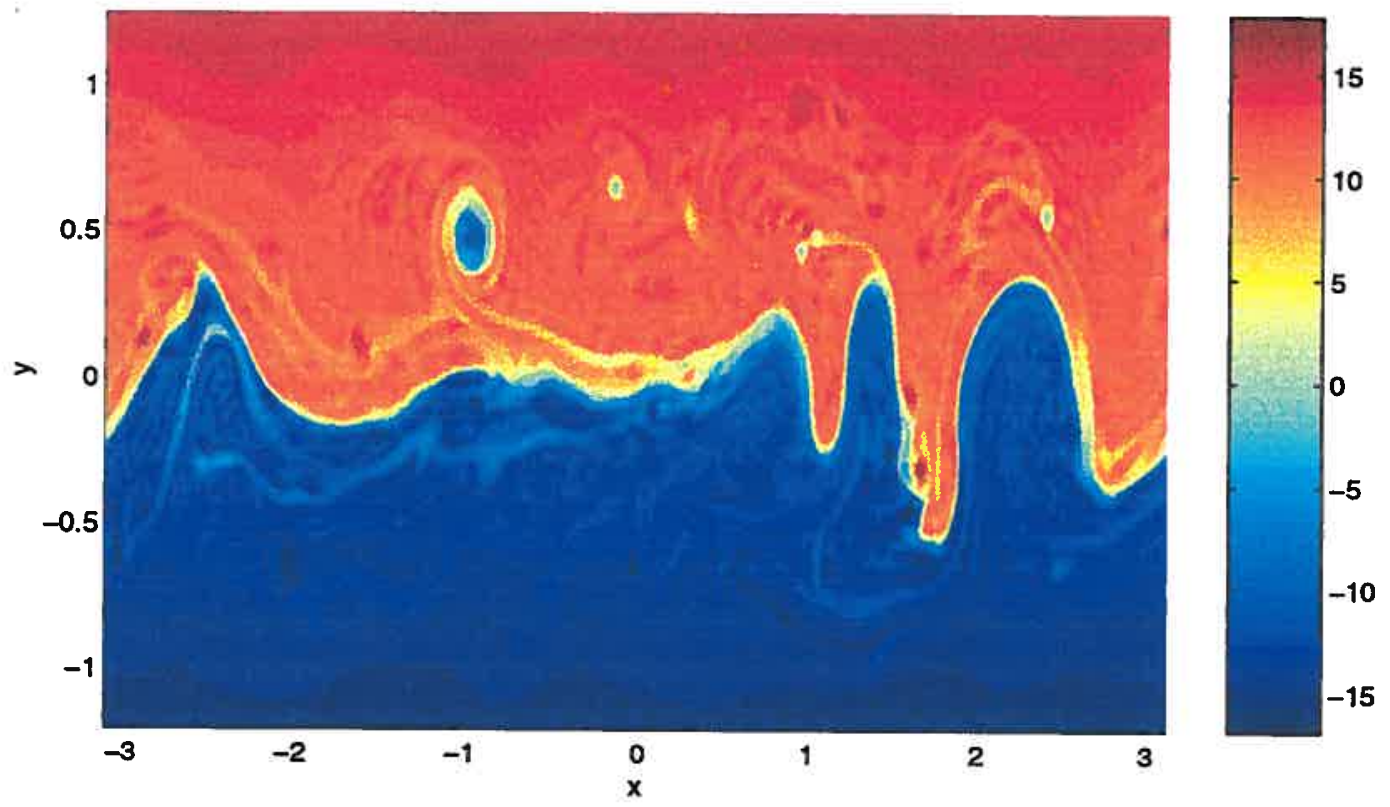

(b)

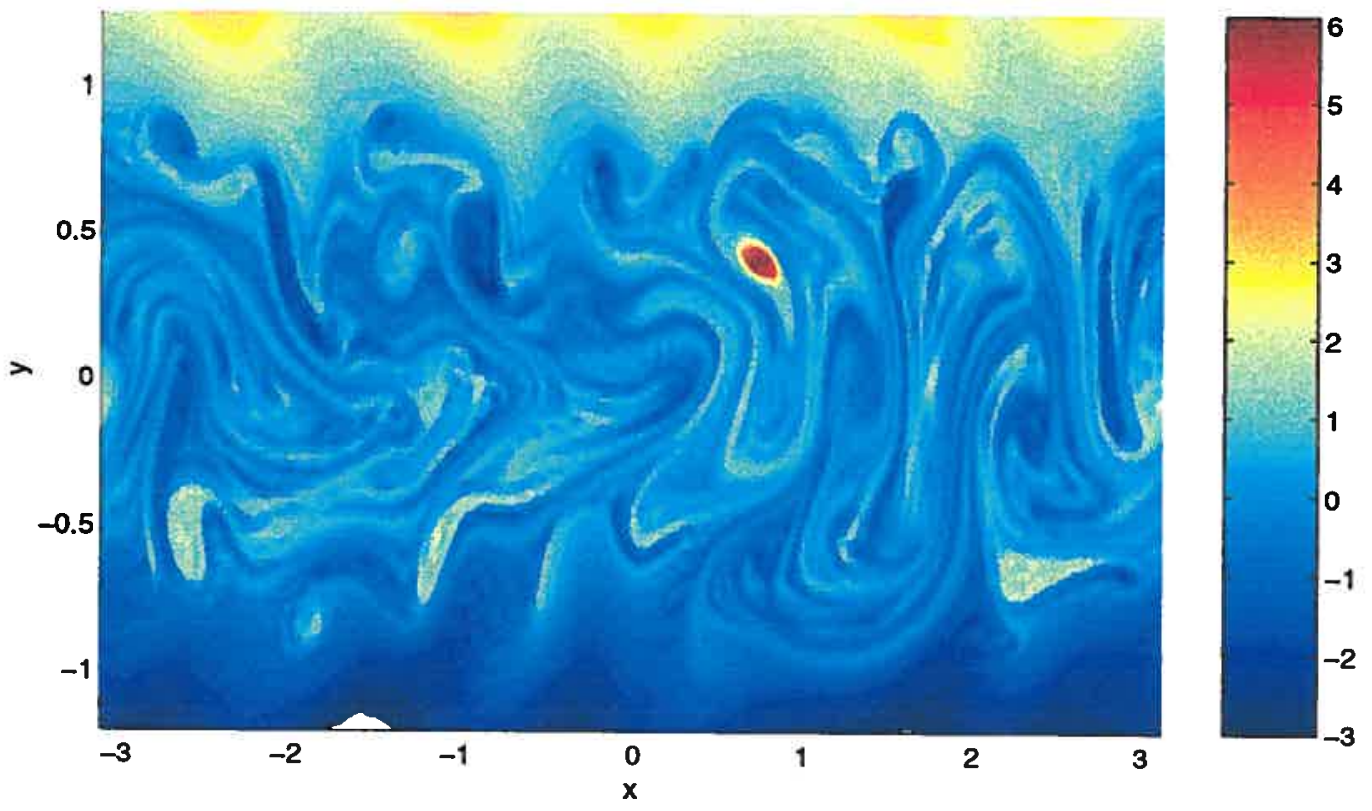

Figure 2-26: (a): Contours of the upper layer potential vorticity at $t=4$ months. (b): Same for the lower layer. Parameters: $u_{1}=1, u_{2}=0, \beta=4.15, \gamma=10.18, \delta=$ $0.25, U=0.8 \mathrm{~m} \mathrm{sec}^{-1}$. 
(a)

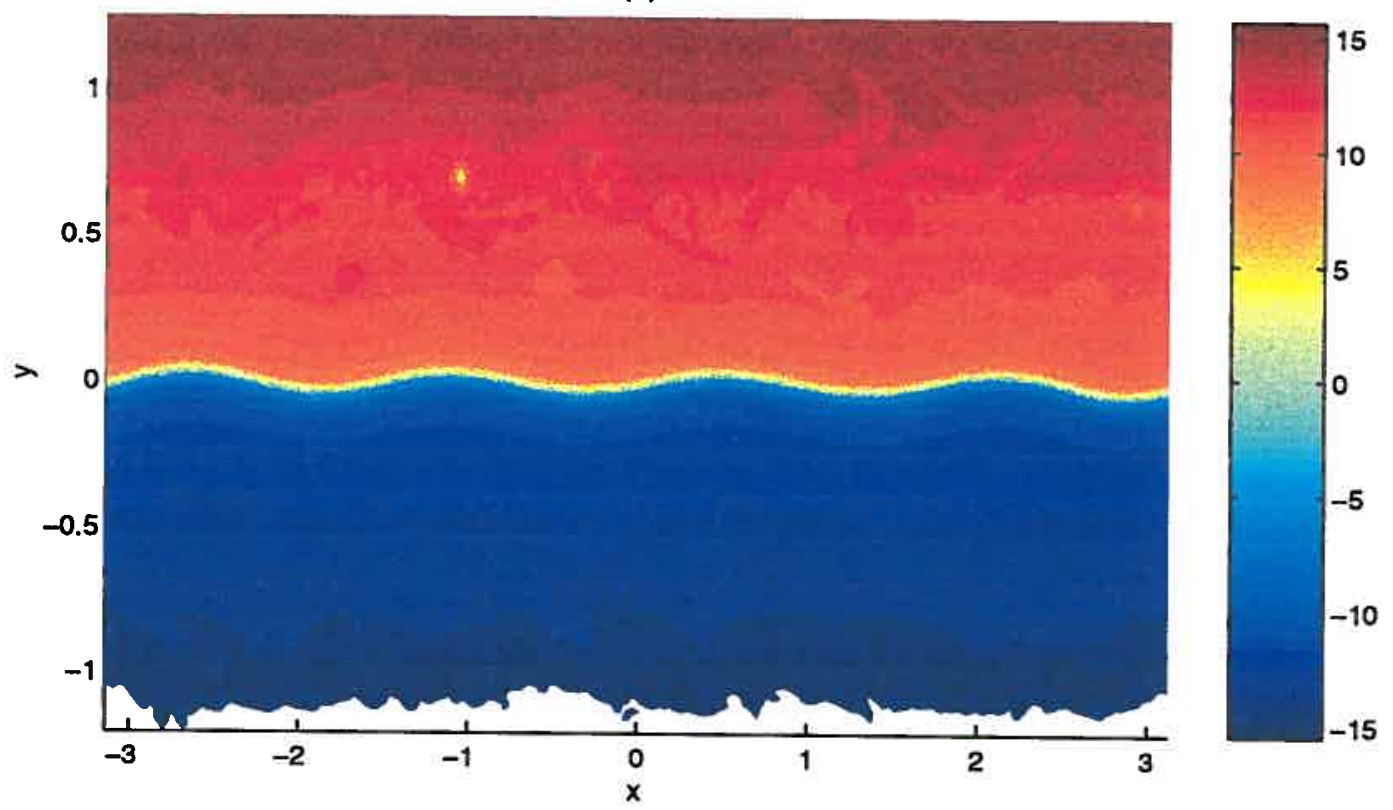

(b)

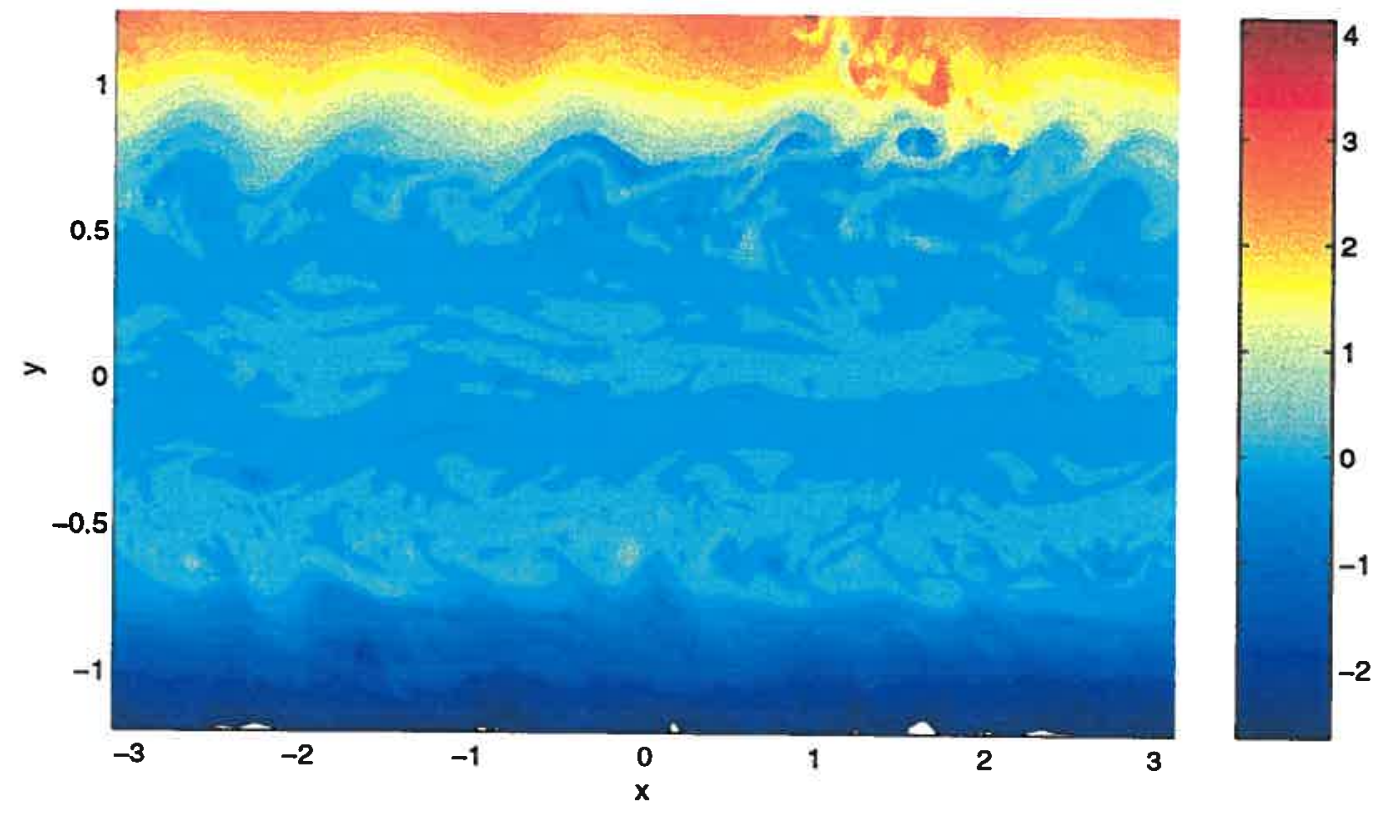

Figure 2-27: (a): Contours of the upper layer potential vorticity at $t=30$ months. (b): Same for the lower layer. Parameters: $u_{1}=1, u_{2}=0, \beta=4.15, \gamma=10.18, \delta=$ $0.25, U=0.8 \mathrm{~m} \mathrm{sec}^{-1}$. 


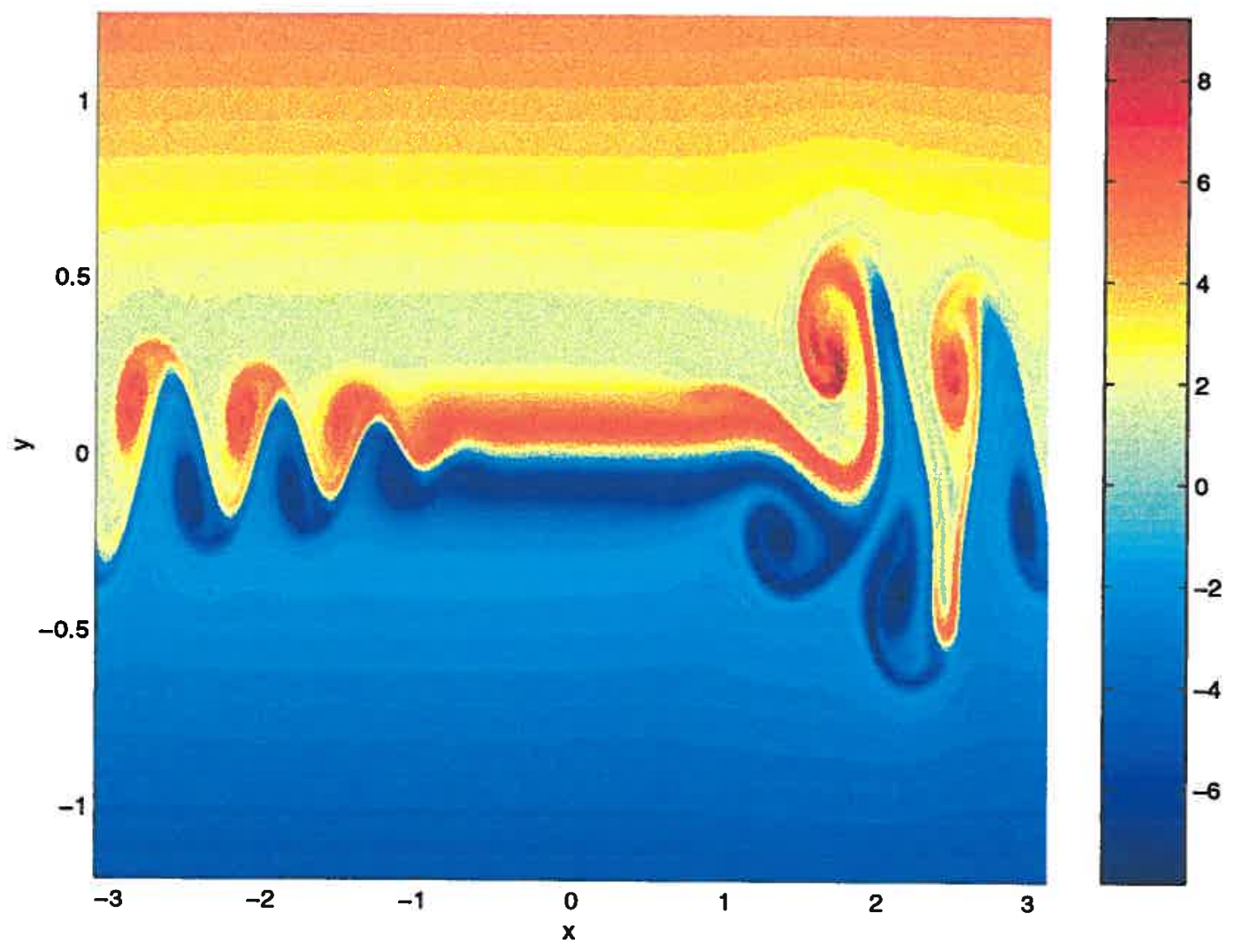

Figure 2-28: Contours of the upper layer potential vorticity at $t=18$ days. Parameters: $u_{1}=1, u_{2}=0.99, \beta=4.15, \gamma=10.18, \delta=0.25, U=0.8 \mathrm{~m} \mathrm{sec}^{-1}$. 


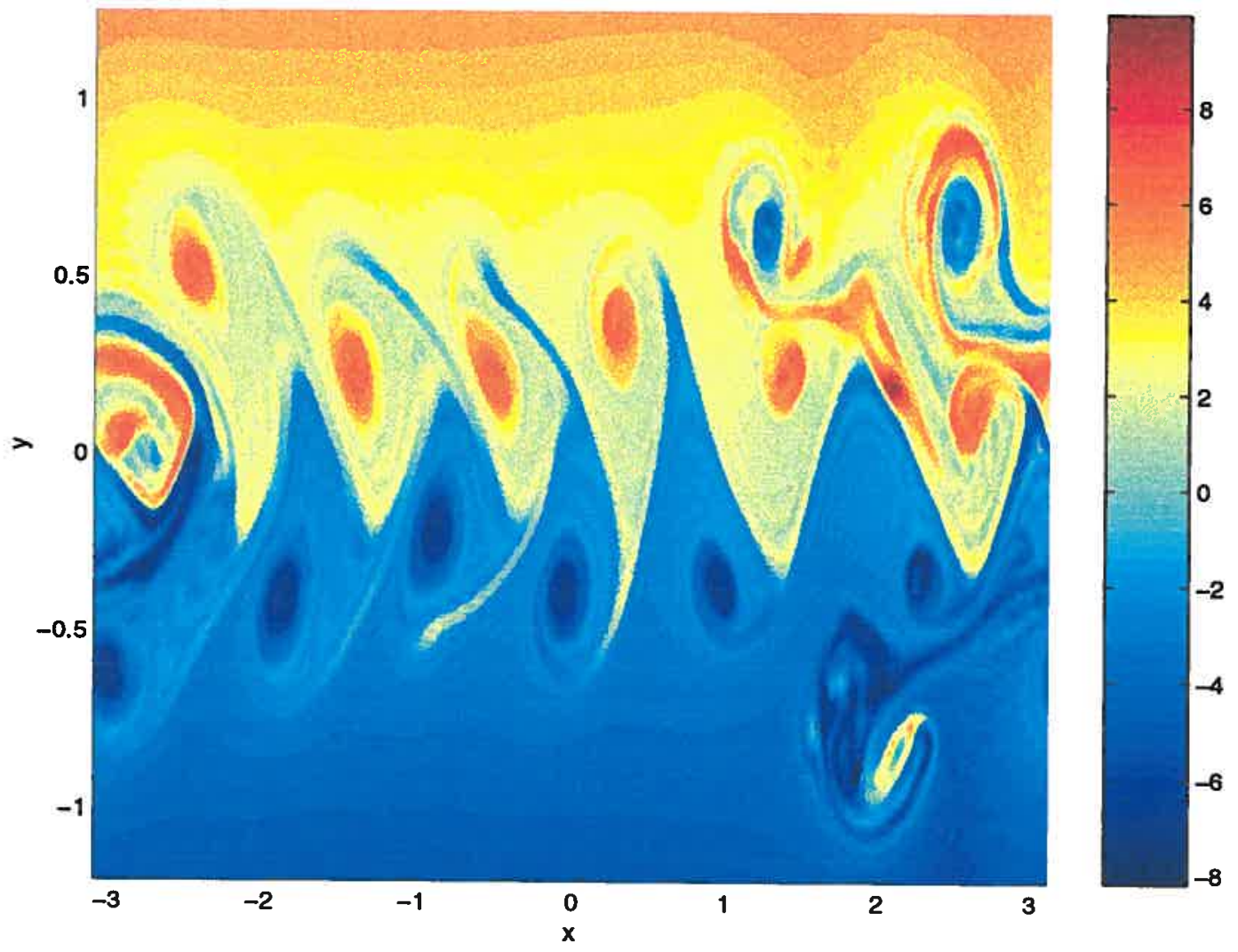

Figure 2-29: Contours of the upper layer potential vorticity at $t=47$ days. Parameters: $u_{1}=1, u_{2}=0.99, \beta=4.15, \gamma=10.18, \delta=0.25, U=0.8 \mathrm{~m} \mathrm{sec}^{-1}$. 


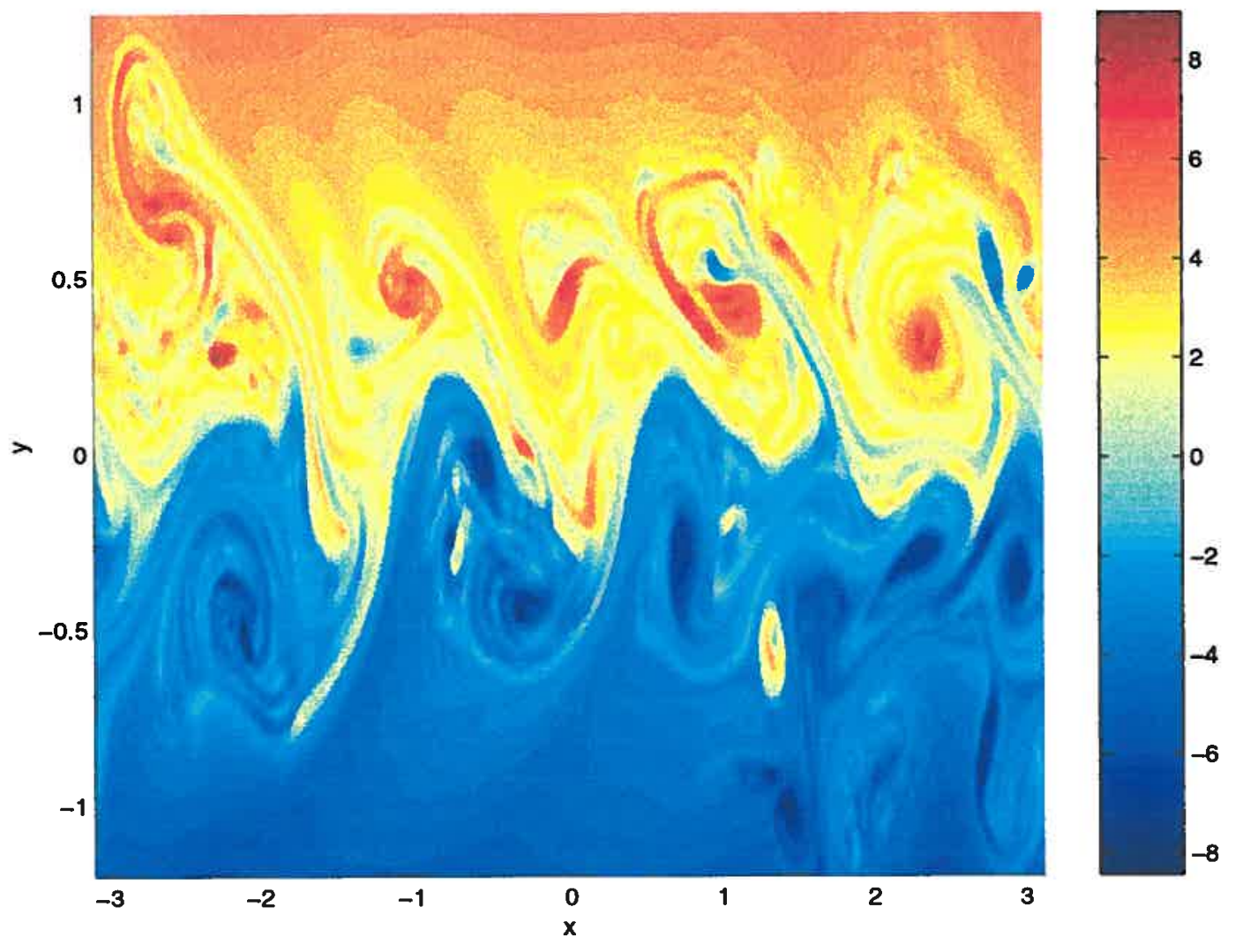

Figure 2-30: Contours of the upper layer potential vorticity at $t=3$ months. Parameters: $u_{1}=1, u_{2}=0.99, \beta=4.15, \gamma=10.18, \delta=0.25, U=0.8 \mathrm{~m} \mathrm{sec}^{-1}$. 


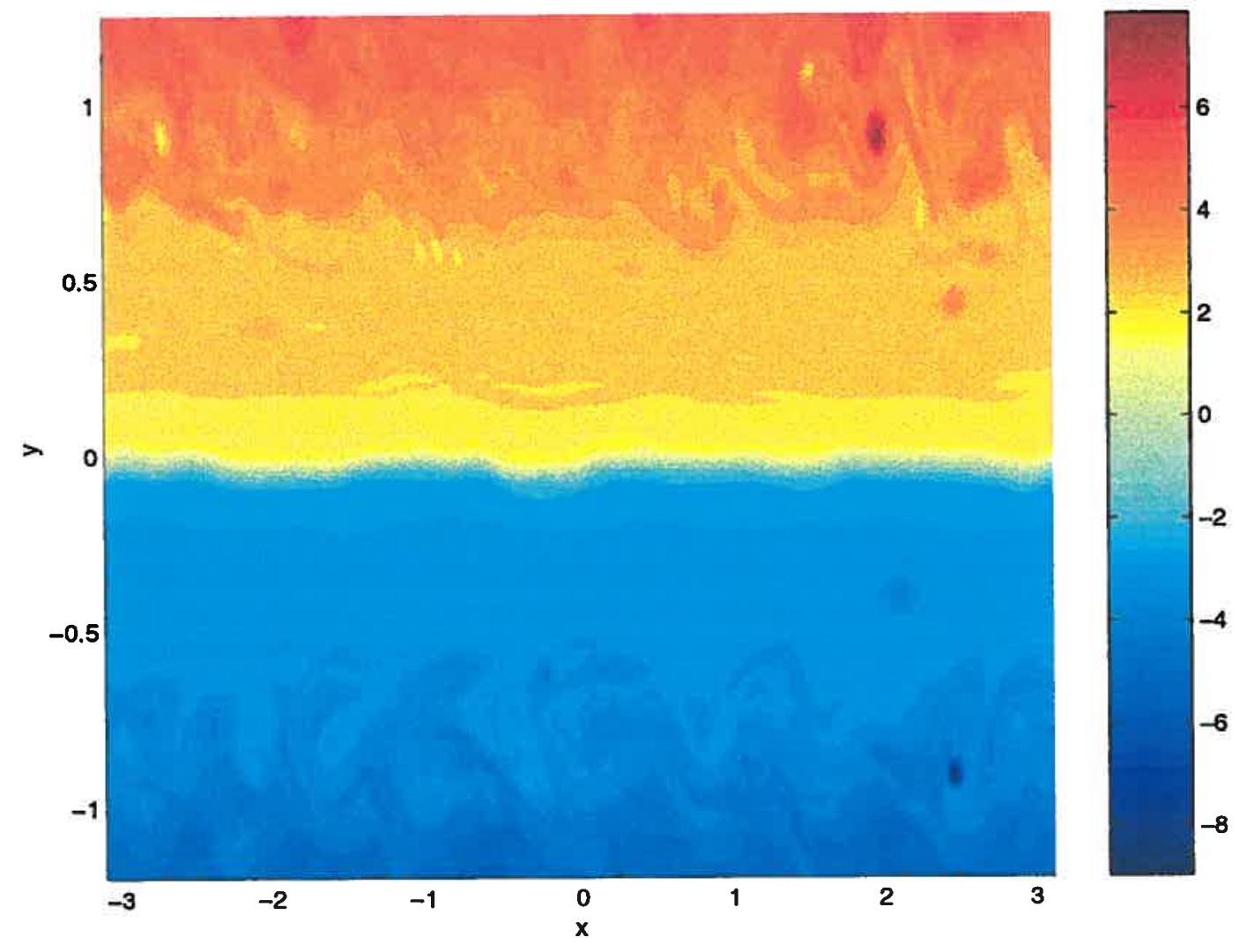

Figure 2-31: Contours of the upper layer potential vorticity at $t=30$ months. $\mathrm{Pa}$ rameters: $u_{1}=1, u_{2}=0.99, \beta=4.15, \gamma=10.18, \delta=0.25, U=0.8 \mathrm{~m} \mathrm{sec}^{-1}$. 
the south by the zones of westward flows with homogenized potential vorticity. Interestingly, the value of the positive $q$-gradient decreased only by $10 \%$ during the flow evolution, which indicates very weak mixing across the upper layer potential vorticity front and intense mixing on the flanks of the jet.

For the case of a strongly barotropic jet, $\left(u_{1}, u_{2}\right)=(1,0.99)$, the regions of initial negative potential vorticity gradient (Figure 2-23a) are removed after equilibration (Figure 2-23b). The final $q$-structure consists of a zone of positive potential vorticity gradient on the axis of the jet with the regions of homogenized potential vorticity north and south of it. In contrast with a strongly baroclinic case, the value of positive $q$-gradient decreased by $45 \%$, which suggests that in a strongly barotropic flow, mixing occurred not only on the flanks of the jet, but also across the potential vorticity front on the axis of the jet.

The differences in potential vorticity mixing are illustrated in Figures 2-24-231. For a strongly baroclinic case, $\left(u_{1}, u_{2}\right)=(1,0)$, a large positive potential vorticity gradient in the upper layer jet appears as an efficient barrier to mixing; even though meanders grow to large amplitudes, the front stays quite coherent and breaks only in a few places giving birth to rings (Figures 2-24, 2-25). Therefore, in the upper layer, mixing occurs on the sides of the jet; potential vorticity filaments get strained and stirred on each side of a meander. Most of $q$-mixing occurs in the lower layer, where the initial potential vorticity gradient is negative. After 4 months of integration (Figure 2-26), the amplitudes of the upper layer meanders decrease, and straining of the potential vorticity contours in the lower layer continues. Both layers are dominated by heton-like structures. The equilibrated field (Figure 2-27) is characterized by homogenized lower layer potential vorticity under the axis of the jet and by a strong $q$-front in the upper layer.

In the strongly barotropic case $\left(u_{2}=0.99\right)$, the meanders start breaking long before they propagate across the domain (Figure 2-28). After 1 month of integration (Figure 2-29), there is an intense potential vorticity mixing across the front and on 
its sides. The front becomes completely broken after 3 months (Figure 2-30). Even though initially there is a strong potential vorticity gradient in the middle of the domain, which should serve as a barrier to mixing, the eddy field is so intense and violent that it eventually breaks the front. This contrasts with a strongly baroclinic case described earlier. The equilibrated potential vorticity field (Figures 2-31) has a positive gradient, but it is much weaker than the initial one. Two regions of homogenized $q$ are located north and south of the front.

\section{Energy and Momentum Constraints}

During a free spin down, why does the flow equilibrate itself by developing the recirculations? The equilibration process, which we view as potential vorticity mixing, must obey the conservation of both momentum and energy. One should remember that although the model is unforced, the conservation is approximate due to the presence of a numerical filter and the sponge layers.

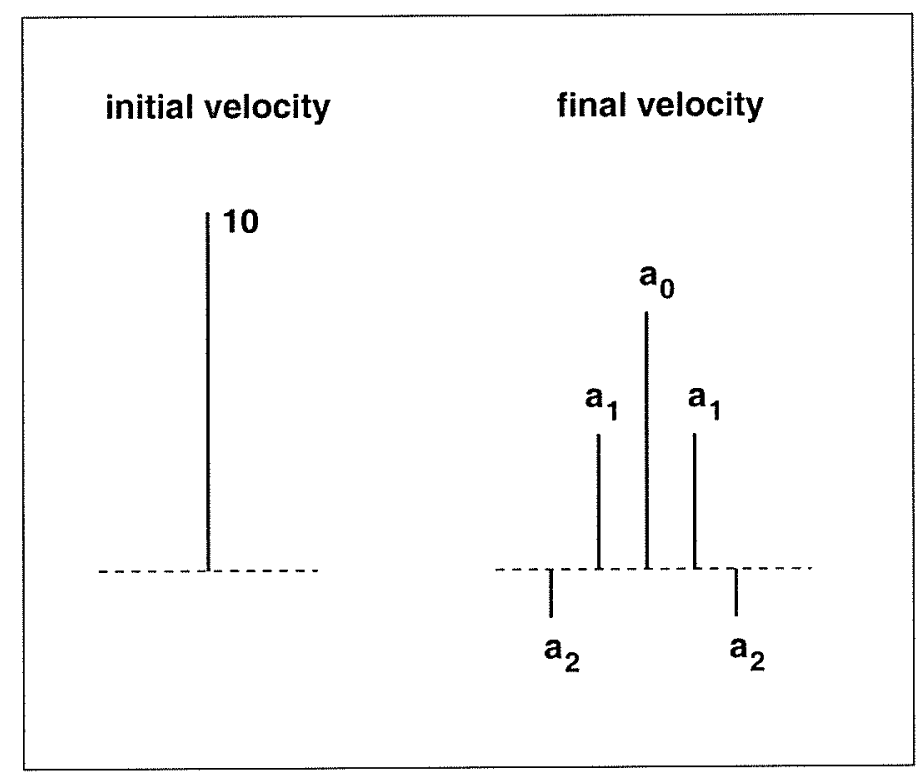

Figure 2-32: A schematic, illustrating a thought experiment. Initial velocity profile (left) and equilibrated velocity profile (right). 
To illustrate the importance of the above constraints, let us consider the following thought experiment. Suppose that an initial barotropically unstable "point" eastward jet has a velocity amplitude of 10 units and the kinetic energy of $\frac{10^{2}}{2}$ units (Figure 2-32, left). Due to potential vorticity mixing, the equilibrated flow can be represented by a broadened jet with velocity amplitudes $\left\{a_{2}, a_{1}, a_{0}, a_{1}, a_{2}\right\}$, where $a_{2}<a_{1}<a_{0}$ (Figure 2-32, right). Note that we make no assumptions about the sign of the amplitude components, $a_{2}$. The conservation of momentum and energy imply:

$$
\left\{\begin{array}{l}
a_{0}+2\left(a_{1}+a_{2}\right)=10, \\
a_{0}^{2}+2\left(a_{1}^{2}+a_{2}^{2}\right)=10^{2} .
\end{array}\right.
$$

Figure 2-33 shows the solutions of the above equations, when the center amplitude, $a_{0}$, is considered as an independent parameter varying between 0 and 10 . This simple experiment shows that the equilibrated jet structure must have negative velocity amplitudes, $a_{2}$, on the flanks of the equilibrated barotropic jet in order to conserve both energy and momentum.

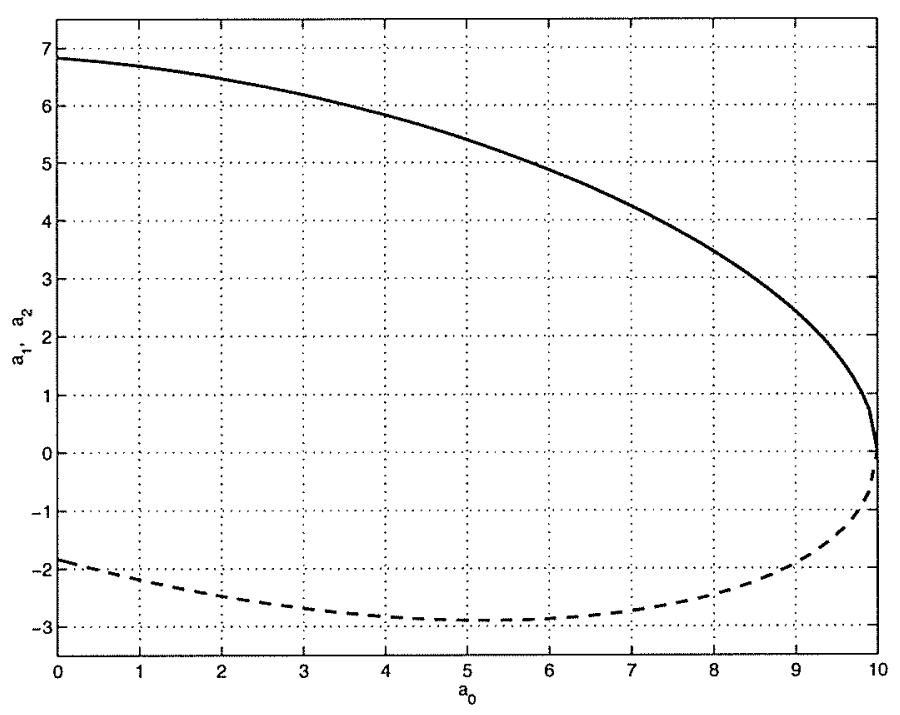

Figure 2-33: The solutions of system of equations (2.27) conserving momentum and energy. The velocity amplitudes $a_{1}$ (solid line) and $a_{2}$ (dashed line) are shown as functions of central velocity amplitude, $a_{0}$. 
(a)

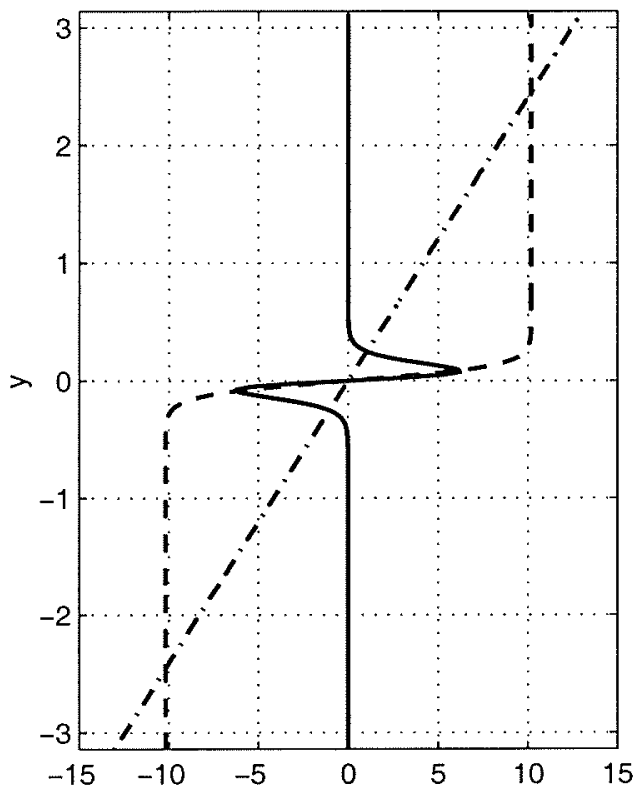

(b)

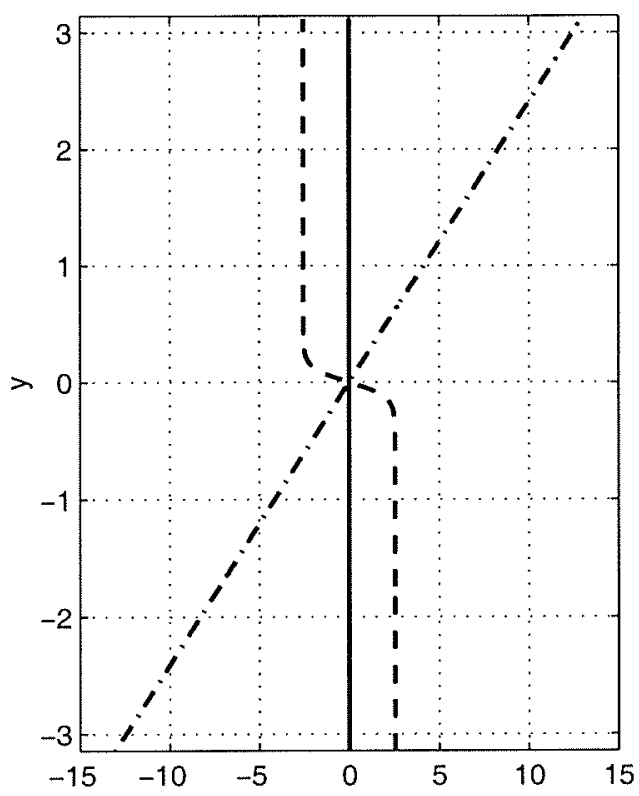

(c)

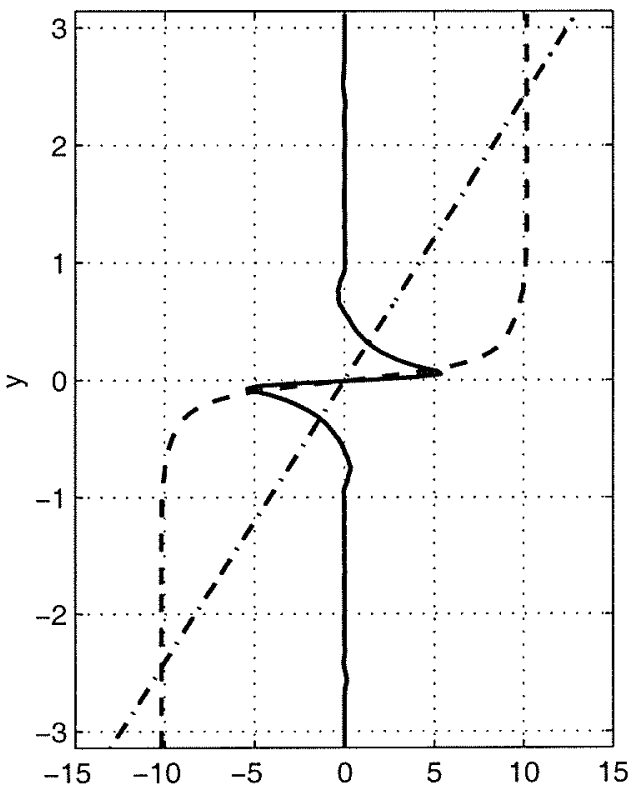

(d)

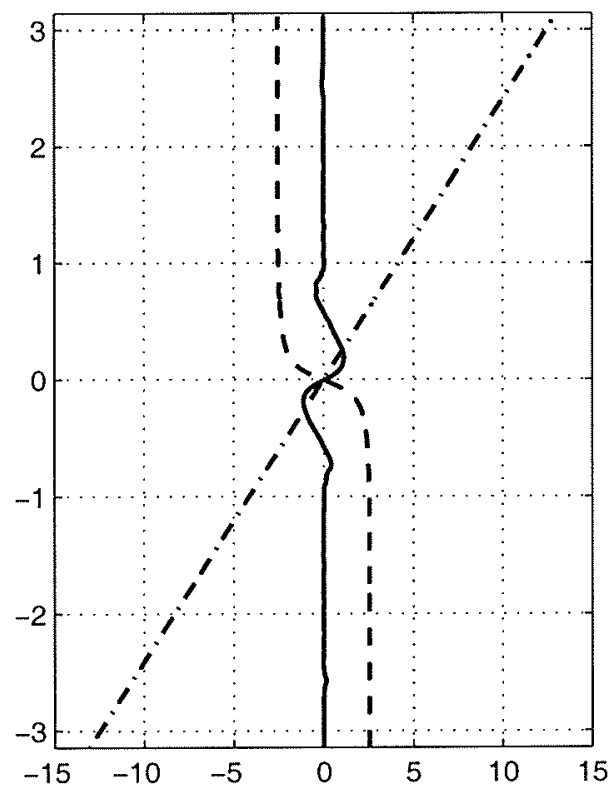

Figure 2-34: Zonally averaged relative vorticity, $\overline{\Psi_{1 y y}}$, (solid), stretching term, $F(1) *$ $\overline{\left(\Psi_{2}-\Psi_{1}\right)}$, (dash) and planetary potential vorticity, $\beta y$, (dash-dot) in the upper layer at the initial (a) and final (b) time. Similar terms for the lower layer at the initial (c) and final (d) time. Parameters: $u_{1}=1, u_{2}=0, \beta=4.15, \gamma=10.18, \delta=0.25, U=$ $0.8 \mathrm{~m} \mathrm{sec}^{-1}$. 
(a)

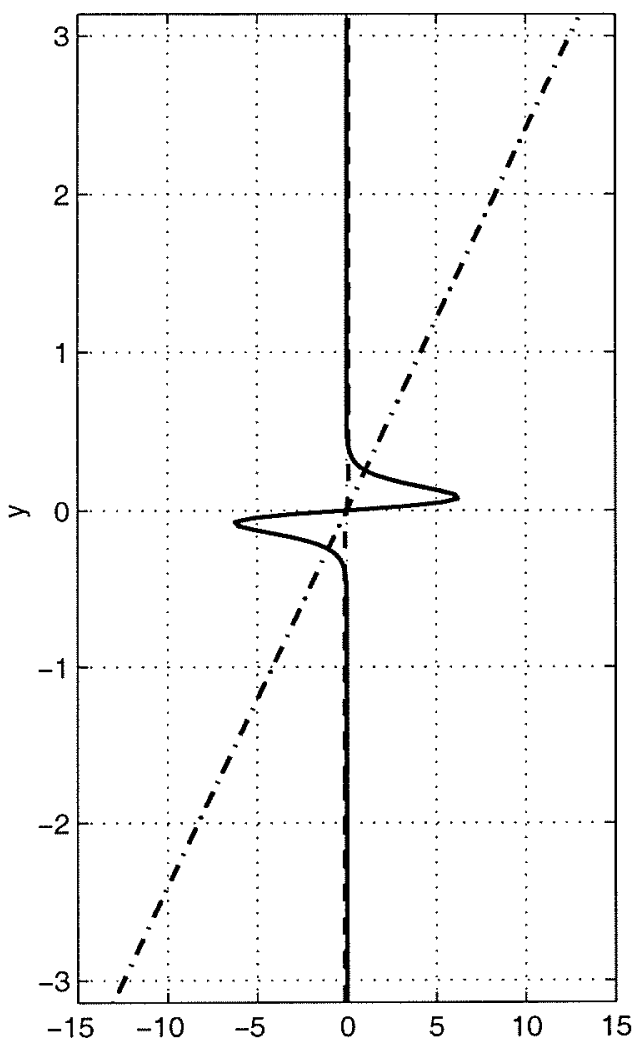

(b)

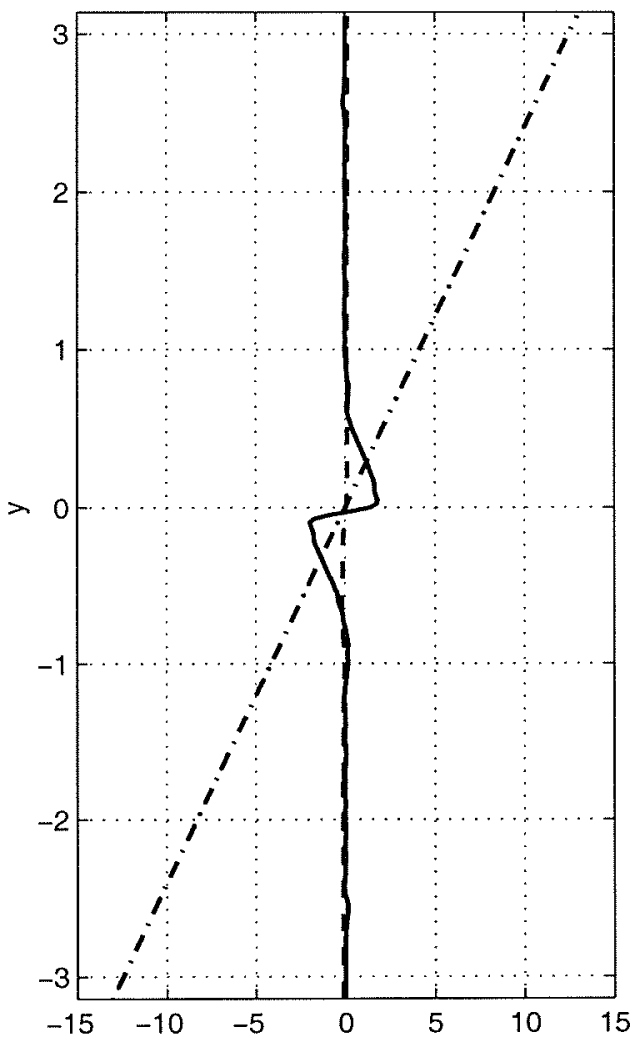

Figure 2-35: Zonally averaged relative vorticity, $\overline{\Psi_{1 y y}}$, (solid), stretching term, $F(1) *$ $\overline{\left(\Psi_{2}-\Psi_{1}\right)}$, (dash) and planetary potential vorticity, $\beta y$, (dash-dot) in the upper layer at the initial (a) and final (b) time. Parameters: $u_{1}=1, u_{2}=0.99, \beta=4.15, \gamma=$ $10.18, \delta=0.25, U=0.8 \mathrm{~m} \mathrm{sec}^{-1}$. 

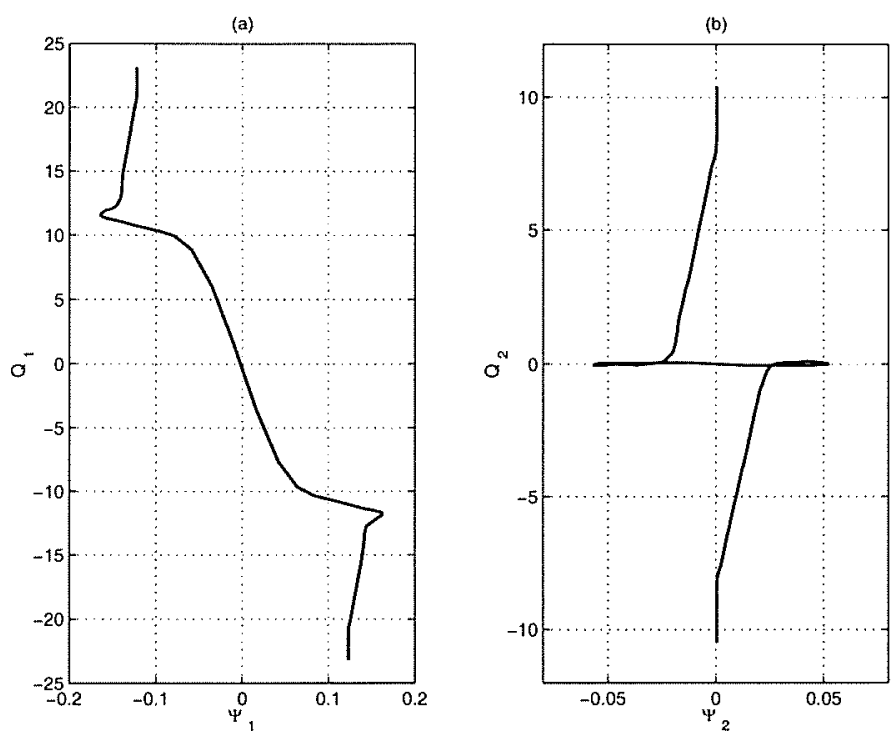

Figure 2-36: Final $\psi-q$ dependence for the upper layer (a) and lower layer (b) when $u_{1}=1, u_{2}=0, \beta=4.15, \gamma=10.18, \delta=0.25, U=0.8 \mathrm{~m} \mathrm{sec}^{-1}$.

\section{Potential Vorticity Components}

The changes in the individual components of potential vorticity fields are presented in Figures 2-34 and 2-35. In strongly baroclinic cases, although the stretching term, $(-1)^{i} F_{i} \overline{\left(\psi_{1}-\psi_{2}\right)}, \quad i=1,2$, varies somewhat, most of the changes come from the zonal relative vorticity, $\overline{\psi_{i y y}}$, in the center of the domain. Therefore, changes in the meridional structure of the flow are mostly responsible for removing the negative values of potential vorticity gradient. In the recirculation regions, the relative vorticity term is zero and the stretching term is constant in $\mathrm{y}$.

\section{$\psi-q$ Relationship for Equilibrated Jet}

Figure 2-36a shows three different regions of final $\psi-q$ relationship in the upper layer for the case of strongly baroclinic initial basic flow. The axis of the jet corresponds to the linear and negative $\psi-q$ dependence. In the zones of recirculations, where the potential vorticity gradient is zero, the $\psi-q$ function is horizontal. The third zone, where $\psi-q$ is positive and linear, is in the area to the north and south of the 


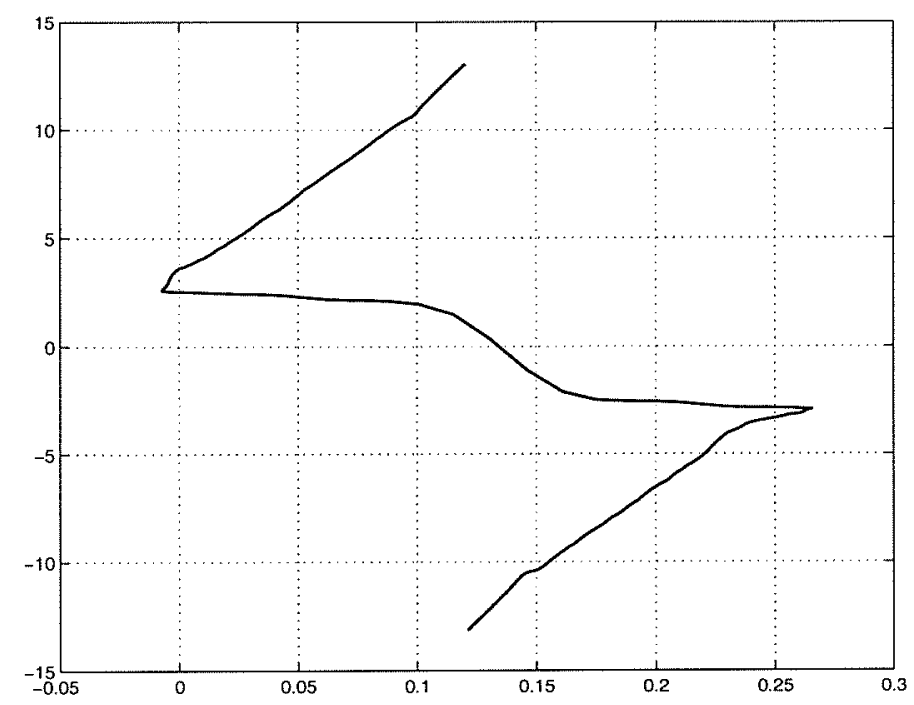

Figure 2-37: Final $\psi-q$ dependence for the upper layer (a) and lower layer (b) when $u_{1}=1, u_{2}=0.99, \beta=4.15, \gamma=10.18, \delta=0.25, U=0.8 \mathrm{~m} \mathrm{sec}^{-1}$.

westward flows. Here, the potential vorticity gradient is equal to $\beta$. In the lower layer (Figure 2-36b), both in the region of eastward jet and westward recirculations, the $\psi-q$ dependence is horizontal. For strongly barotropic initial basic flow (Figure 237), the final $\psi-q$ relationship is similar to that of the upper layer strongly baroclinic case.

\subsubsection{Relative Strength of the Recirculations}

\section{Dependence on Baroclinic Structure}

To understand how the strength of the recirculations depends on the baroclinic structure of the jet for a given $\beta$, we conducted a series of experiments in which the amplitude of the nondimensional upper layer basic velocity was fixed at 1 and the amplitude of the nondimensional lower layer basic velocity was varied between 0 and 0.99 .

Figure 2-38 shows total dimensional net, eastward and westward transports for the equilibrated jet as functions of the lower layer nondimensional velocity amplitude, 


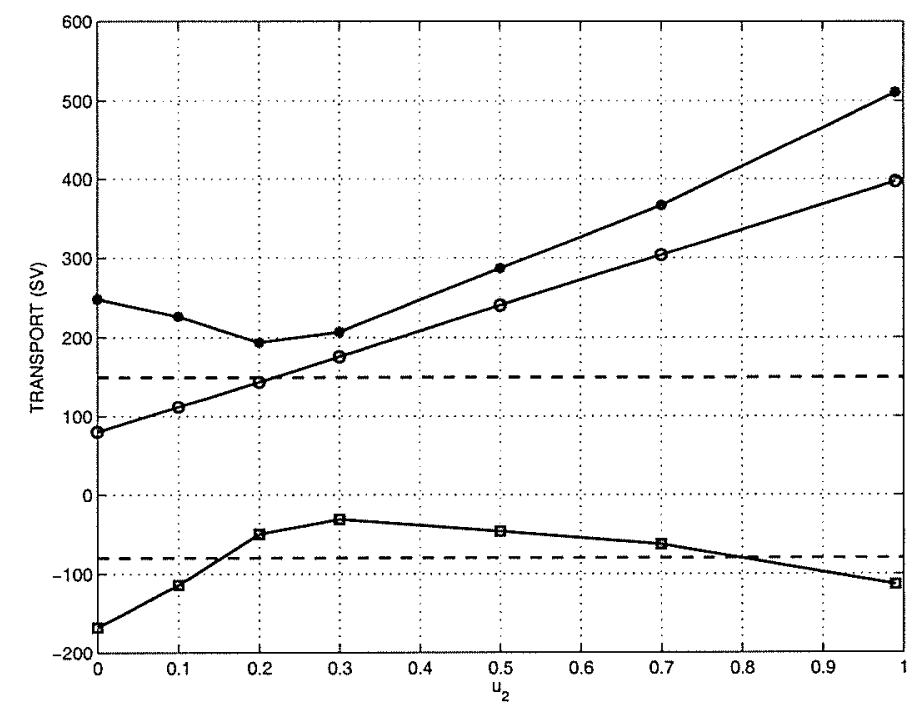

Figure 2-38: Total net (o), eastward $\left({ }^{*}\right)$ and westward $(\square)$ transports (in Sv) for the equilibrated jet as functions of the amplitude of the nondimensional lower layer basic velocity, $u_{2}$. The upper layer nondimensional velocity amplitude, $u_{1}$, was fixed at 1.0. Parameters: $\beta=4.15, \gamma=10.18, \delta=0.25, U=0.8 \mathrm{~m} \mathrm{sec}^{-1}$. Dashed lines show the observed transport in the Gulf Stream ( $150 \mathrm{~Sv})$ and in the recirculations ( $-80 \mathrm{~Sv})$.

$u_{2}$, for $\beta=4.15$. Both eastward and westward transports were not monotonic. For $u_{2}<0.3$, the eastward transport decreased from $250 \mathrm{~Sv}$ to $200 \mathrm{~Sv}$, whereas the westward transport diminished from $180 \mathrm{~Sv}$ to $30 \mathrm{~Sv}$. For $u_{2}>0.3$, they both increased to $500 \mathrm{~Sv}$ and $110 \mathrm{~Sv}$ correspondingly. Note that the observed transports in the Gulf Stream and its recirculations correspond to $150 \mathrm{~Sv}$ and $80 \mathrm{~Sv}$ (Hogg [12]).

Similar non-monotonic dependence of the recirculations strength on the lower layer nondimensional velocity amplitude was observed for three different values of nondimensional $\beta$ (Figure 2-39) corresponding to three different velocity scales: $U=$ $0.6,0.8$ and $1.0 \mathrm{~m} \mathrm{sec}^{-1}$. The minimum in the strength of the recirculations was attained at $u_{2}=0.3$ for $\beta=4.15$ and $\beta=5.53$ and at $u_{2}=0.5$ for $\beta=3.32$. Therefore, the recirculations were strongest for strongly baroclinic initial basic flows, weakest for flows with intermediate baroclinicity and of medium strength for strongly barotropic flows. 


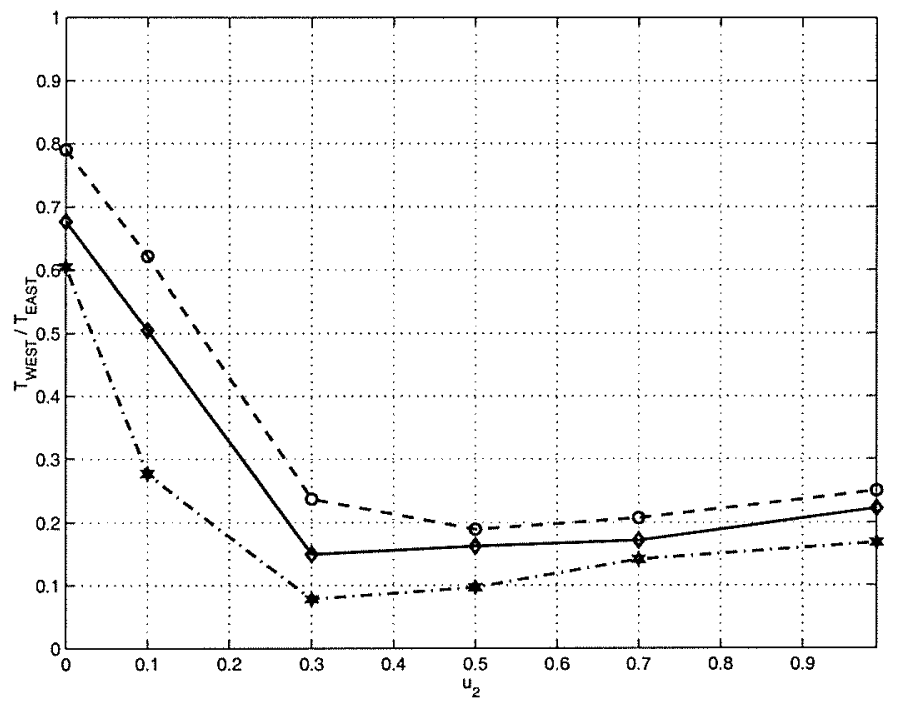

Figure 2-39: The relative strength of the recirculations, defined by the ratio of total westward to eastward transports, as a function of the lower layer nondimensional velocity amplitude, $u_{2}$. Parameters: $u_{1}=1, \gamma=10.18, \delta=0.25$. The dashed, solid and dashed-dotted lines correspond to $\beta=3.32, U=1 \mathrm{~m} \mathrm{sec}^{-1}, \beta=4.15, U=0.8$ $\mathrm{m} \mathrm{sec}{ }^{-1}$ and $\beta=5.53, U=0.6 \mathrm{~m} \mathrm{sec}^{-1}$ respectively.
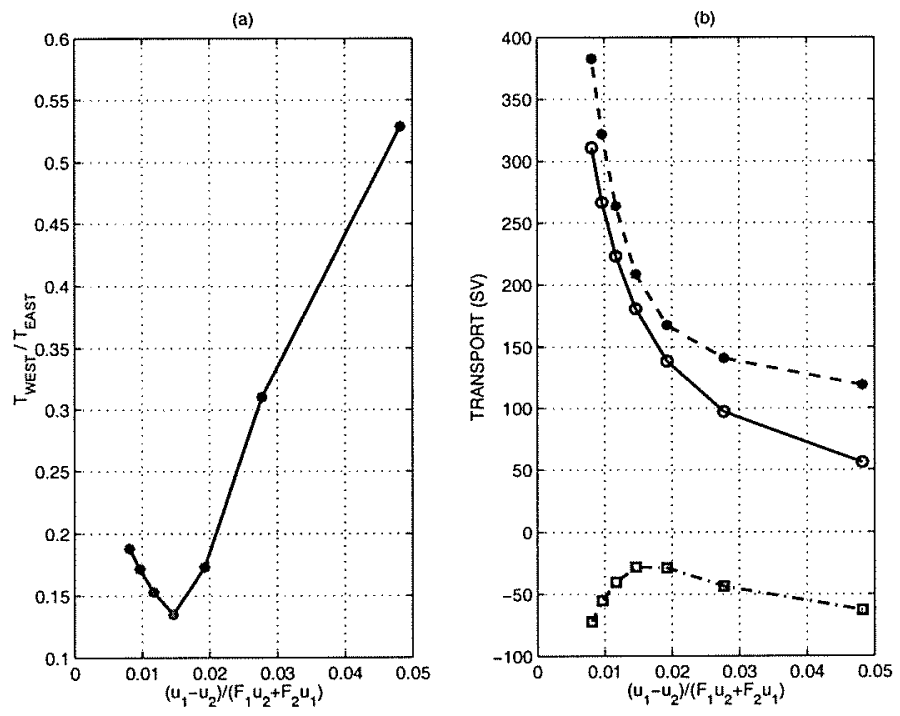

Figure 2-40: (a): The ratio of the total westward to the total eastward transport as a function of a baroclinic parameter, $\frac{u_{1}-u_{2}}{F_{1} u_{2}+F_{2} u_{1}}$. (b): The dimensional total net (solid line), westward (dashed-dotted line) and eastward transports (dashed line) as functions of a baroclinic parameter. Parameters: $\beta=4.15, \gamma=10.18, \delta=0.25$. 


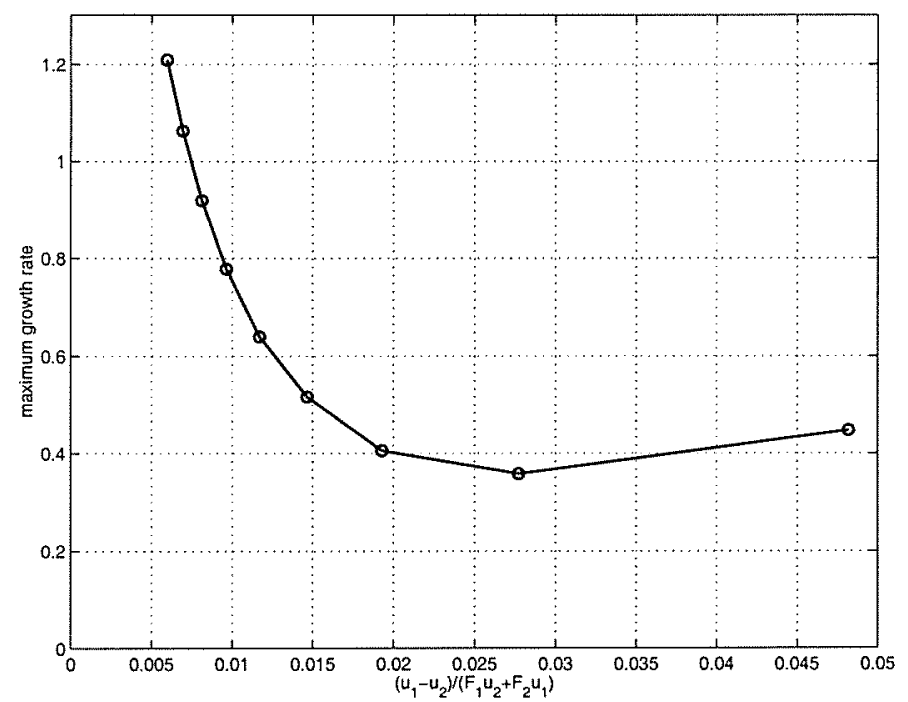

Figure 2-41: Maximum linear growth rate as a function of a baroclinic parameter, $\frac{u_{1}-u_{2}}{F_{1} u_{2}+F_{2} u_{1}}$. Parameters: $\beta=4.15, \gamma^{2}=103.75, \delta=0.25$.

However, the above experiments differed in their total energy levels. To see if the existence of the local minimum in the recirculation strength could be related to changes in the total energy level or in the partition of kinetic to potential energies, a set of experiments was conducted where the total energy was fixed and only the baroclinicity of the basic flow was changed. The latter was defined as the ratio of the basic baroclinic velocity, $u_{1}-u_{2}$, to the basic barotropic velocity, $F_{1} u_{2}+F_{2} u_{1}$. Figure 2-40a shows the resulting relative strength of the recirculations. There is still a minimum reached at the value of the baroclinic parameter that corresponds to $u_{1}=1.0$ and $u_{2}=0.3$. The corresponding partition of kinetic to potential energies was a monotonic function of the baroclinic parameter. The total dimensional transport (Figure 2-40b) also indicates that minimum absolute value of the westward transport is attained at $u_{1}=1.0$ and $u_{2}=0.3$.

Therefore, a non-monotonic behavior of the relative strength of the recirculations is not related to the total energy level or to the kinetic-potential energy partition. Instead, it is determined solely by the baroclinic structure of the initial basic flow. Linear stability analysis reported in section 2.2.3 showed that the growth rate was a 
non-monotonic function of the lower layer nondimensional velocity, $u_{2}$, for a fixed $u_{1}$ and $\beta$ (Figure 2-6). The shape of the growth rate curves indicated the importance of a baroclinic mode when $u_{2}$ was close to zero and of a barotropic mode when $u_{2}$ was close to $u_{1}$. For the intermediate values of $u_{2}$, the growth rate was minimum, and both modes had similar growths. Also, in the experiments with the fixed total energy level, maximum growth rate was a non-monotonic function of a baroclinic parameter, $\frac{u_{1}-u_{2}}{F_{1} u_{2}+F_{2} u_{1}}$ (Figure 2-41).

Linear stability analysis also showed that recirculation development due to linear wave-mean flow interaction was possible only for strongly baroclinic flows. An eddy-mean flow interaction model applied to a strongly baroclinic basic flow produced recirculations in both layers. The same procedure applied to a strongly barotropic flow did not result in flow equilibration. Therefore, for strongly baroclinic basic flows, eddy-mean flow interaction alone can generate recirculations in both layers through residual meridional circulation. Thus, the results of the nonlinear calculations showing a decrease in the relative strength of the recirculations with an increase in $u_{2}$ for strongly baroclinic jets are in agreement with the results of the linear stability analysis.

On the other hand, recirculations developed in the process of a nonlinear equilibration of a strongly barotropic flow. Clearly, nonlinear eddy-eddy and eddy-mean flow interactions were necessary for their generation. Therefore, the principle mechanism leading the recirculation development is different in the case of strongly baroclinic and strongly barotropic flows.

In the present study, we did not perform a weakly-nonlinear analysis of the basic state flows. However, Flierl and Meacham (personal communication) showed that in a contour dynamical model of a two-layer cusp jet with three potential vorticity fronts in each layer, the coefficient in the amplitude equation changes sign for the intermediate values of the baroclinic parameter, indicating that the flow does not saturate. Therefore, equilibration requires a strongly nonlinear regime. Similarly, in 


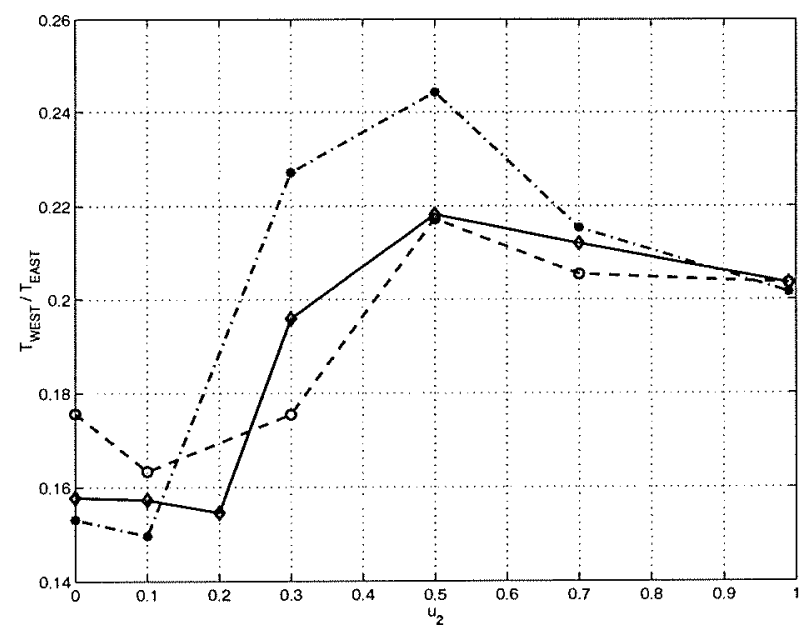

Figure 2-42: The ratio of the upper to the lower layer westward transports for the equilibrated jet, as a function of the lower layer nondimensional velocity amplitude, $u_{2}$. Parameters: $u_{1}=1, \gamma=10.18, \delta=0.25$. The dashed, solid and dashed-dotted lines correspond to $\beta=3.32, U=1 \mathrm{~m} \mathrm{sec}^{-1}, \beta=4.15, U=0.8 \mathrm{~m} \mathrm{sec}^{-1}$ and $\beta=5.53, U=0.6 \mathrm{~m} \mathrm{sec}^{-1}$ respectively.

a case of continuous potential vorticity gradient, a weakly-nonlinear analysis applied to basic state flows with intermediate baroclinicity might not necessarily give any insight into the equilibration process.

Figure 2-42 presents the ratio of the upper to the lower layer westward transports of the equilibrated jet, as a function of the lower layer nondimensional velocity amplitude, $u_{2}$, for three different values of nondimensional $\beta$. The ratio varied between 0.15 and 0.24 with maximum values reached at the intermediate values of $u_{2}$ and minimum values attained when $u_{2}=0$. Recalling that the layer depths were chosen as $1 \mathrm{~km}$ and $4 \mathrm{~km}$ suggests that lower layer recirculations were always stronger than upper layer recirculations.

\section{Dependence on $\beta$}

The relative strength of the recirculations, defined as the ratio of the total westward to the total eastward transport of the equilibrated jet, increased with a decrease in the value of a nondimensional $\beta$-parameter (Figure 2-39). This result is in agreement 


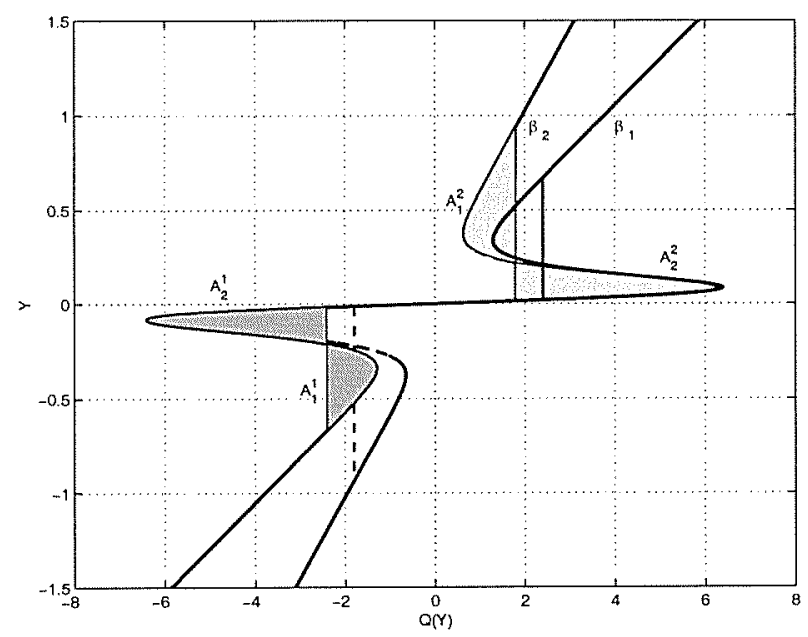

Figure 2-43: Schematic showing mean potential vorticity for two values of nondimensional $\beta: \beta_{1}>\beta_{2}$. Light shaded area indicates mixed $q$ for $\beta_{2}$, dark shaded area indicates mixed $q$ for $\beta_{1}$.

with the findings by Jayne et al. [18], who studied the statistically steady state of the eastward jet and its recirculations driven by the unstable jet inflow on the eastern boundary and by the stable jet outflow though the western boundary.

The increase in the recirculation strength with a decrease in nondimensional $\beta$ can be easily interpreted by viewing the equilibration process as the one of potential vorticity mixing. Figure 2-43 shows schematic potential vorticity profiles for two different values of nondimensional $\beta: \beta_{1}>\beta_{2}$. After equilibration, the regions of negative potential vorticity gradient are removed. Recirculations appearing north and south of the jet correspond to the regions with homogenized potential vorticity. Let us assume here that total enstrophy is conserved and that the potential vorticity gradient on the axis of the jet does not change during equilibration. Then, the following areas must be equal (see Figure): $A_{1}^{2}=A_{2}^{2}$ and $A_{1}^{1}=A_{2}^{1}$. Therefore, the size of the homogenized region is smaller for $\beta=\beta_{1}$, which indicates smaller recirculations. Hence, conservation of enstrophy and symmetry of the flow imply that the region of homogenized potential vorticity for $\beta_{1}$ will be smaller than that for $\beta_{2}$. In the present model, enstrophy is not exactly conserved due to the presence of weak sponge 
layers and a numerical filter; it decreases by less than $5 \%$ during spin down. Also, the potential vorticity gradient corresponding to the jet axis also changes depending on the baroclinicity of the basic flow. However, the above argument illustrates the importance of potential vorticity mixing and approximate enstrophy conservation as constraints on the recirculation size.

\subsection{Results for the Reduced-Gravity Model}

To understand the impact of the barotropic instability on the mean flow and its possible role in generating the recirculations, a linear stability analysis was performed in a framework of a reduced-gravity model. The latter can be considered as a limiting case of a two layer model, described in section 2.1, when the depth of the lower layer is infinite, i.e. $\delta=D_{1} / D_{2}=0$. The upper layer is dynamically active and is governed by the following equation:

$$
\frac{\partial q}{\partial t}+\mathcal{J}(\Psi, q)+\mathcal{J}(\phi, Q)=0
$$

where $\Psi(y)=-\int^{y} U\left(y^{\prime}\right) d y^{\prime}$ and $Q(y)=\beta y-\gamma^{2} \Psi+\frac{d^{2} \Psi}{d y^{2}}$ are the basic flow streamfunction and potential vorticity fields, $\phi=\phi(x, y, t)$ and $q=-\gamma^{2} \phi+\frac{d^{2} \phi}{d y^{2}}$ are the perturbation streamfunction and potential vorticity fields. The model is defined by the following nondimensional parameters: the inverse deformation radius, $\gamma=L / L_{\text {def }}$, and nondimensional beta-parameter, $\beta=\beta_{d i m} L^{2} / U$. Basic state velocity is given by Equation (2.5).

Although the structure of the basic mode is baroclinic, only the mechanism of barotropic instability is present in the reduced-gravity model. Again, we look for a zonally periodic solution of the form

$$
\phi(x, y, t)=\operatorname{Real}\left(\Phi(y) e^{i k(x-c t)}\right)
$$



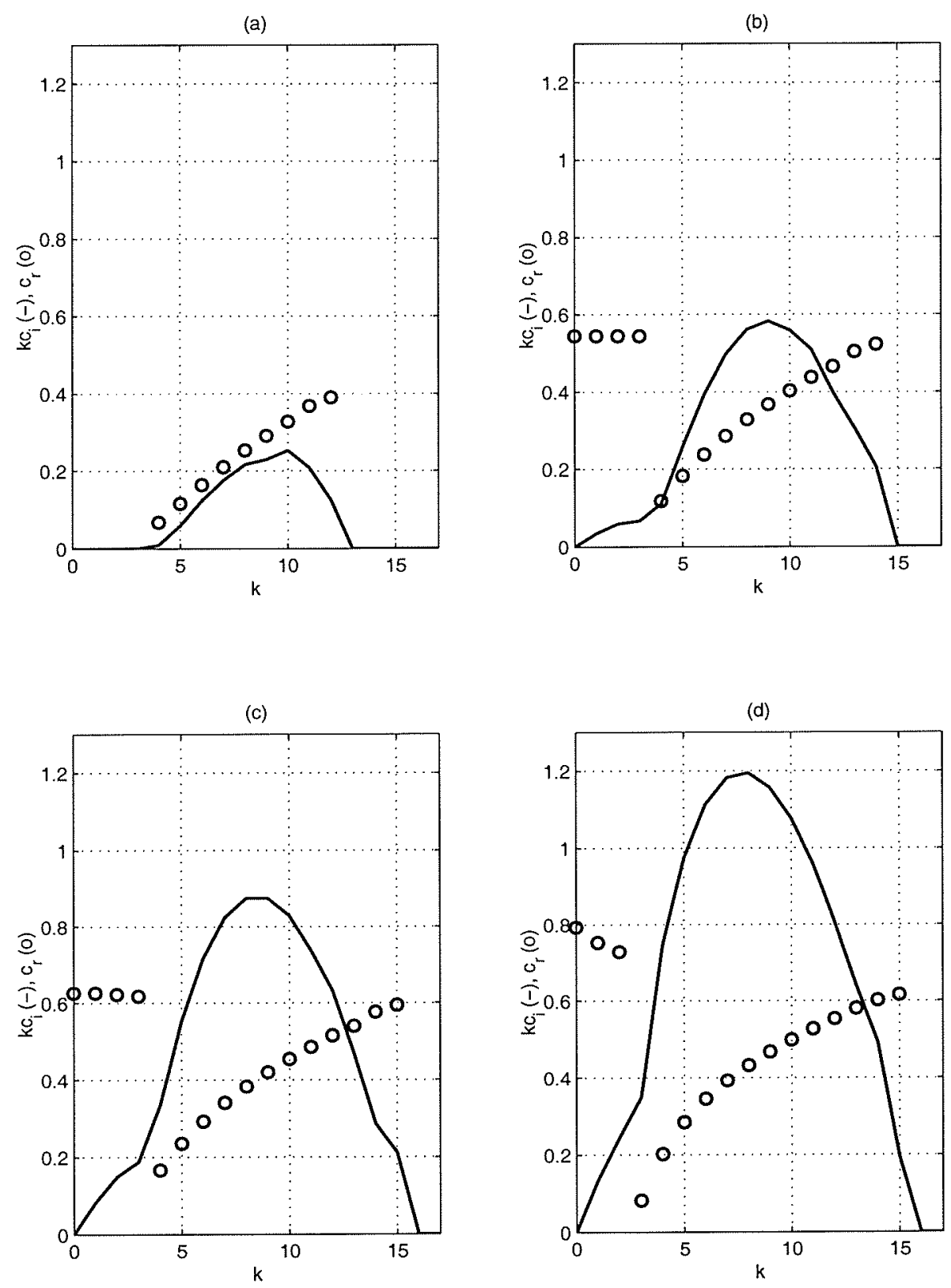

Figure 2-44: The maximum growth rate, $k c_{i}$, (solid line) and the corresponding real part of the phase speed, $c_{r}$, (denoted by circles) as functions of a horizontal wave number, $k$, in the reduced-gravity model for $\gamma=9.11, L_{d e f}=44 \mathrm{~km}$ (a), $\gamma=$ $6.27, L_{d e f}=65 \mathrm{~km}(\mathrm{~b}), \gamma=4.07, L_{d e f}=100 \mathrm{~km}(\mathrm{c})$ and $\gamma=0, L_{d e f}=\infty$ (d). Parameters: $\beta=4.15, w=8.14$. 
(a)

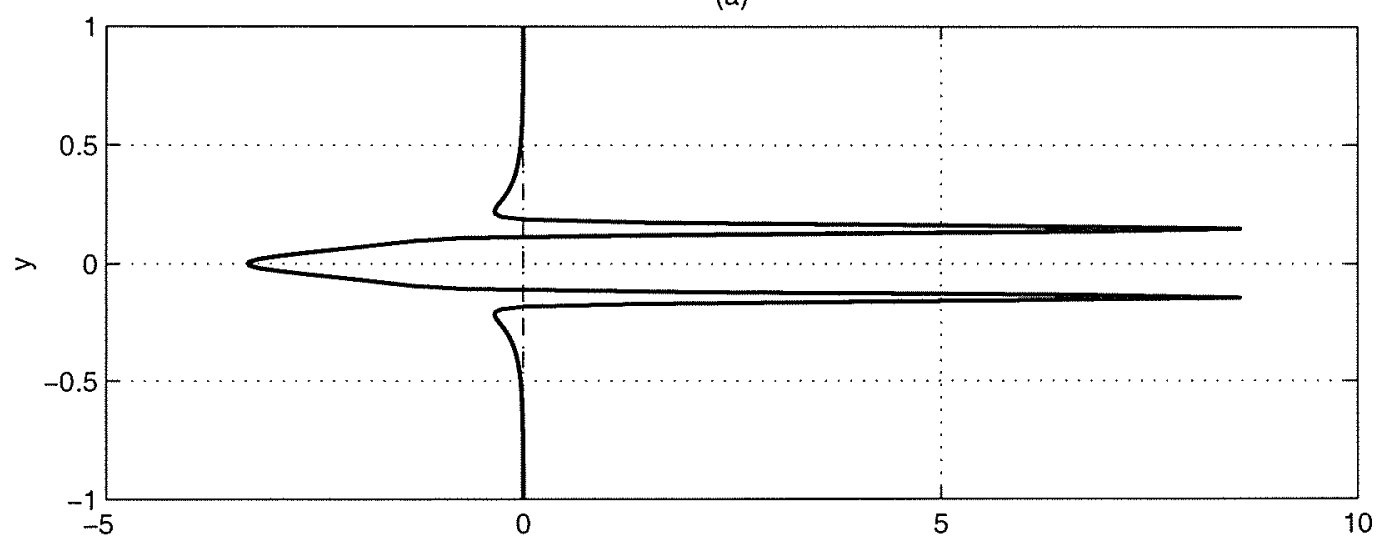

(b)

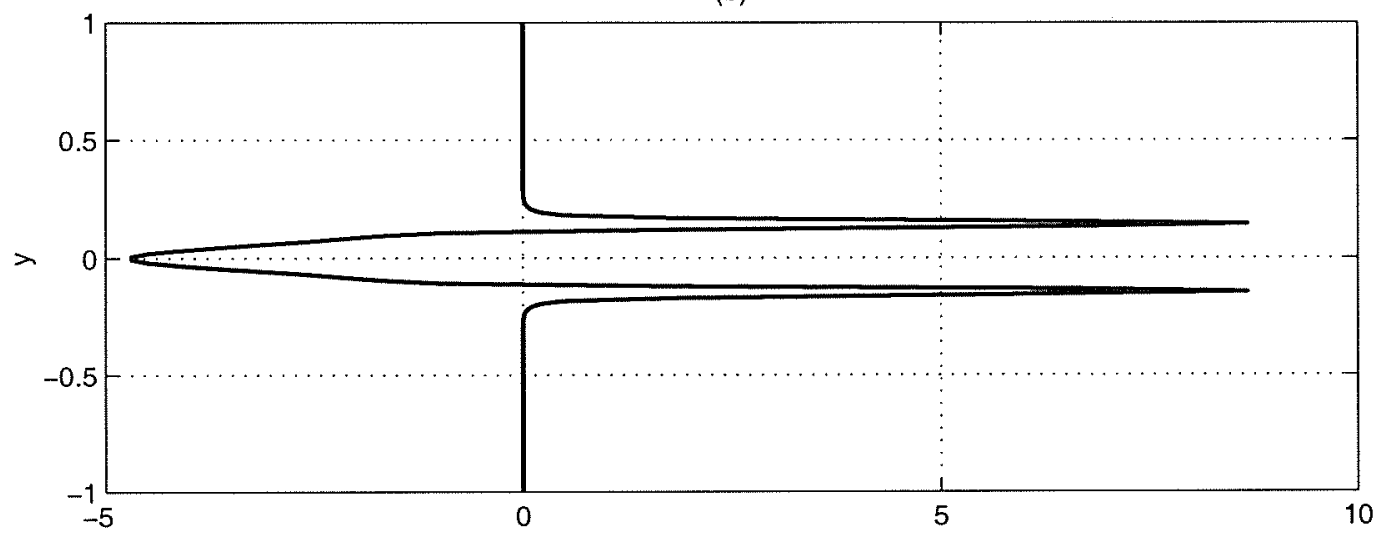

(c)

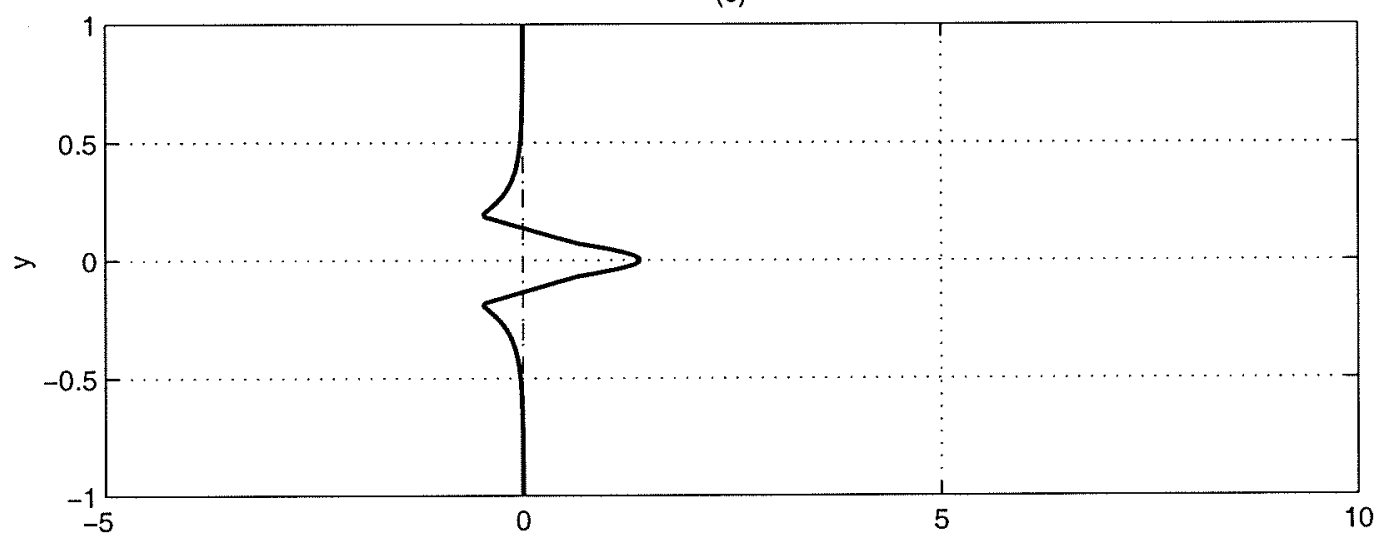

Figure 2-45: Zonal mean acceleration, $\frac{\partial \bar{u}}{\partial t}$, (a), northward potential vorticity flux, $\overline{v q}$, (b) and residual meridional circulation, $\overline{v^{*}},(\mathrm{c})$. Parameters of the reduced-gravity model: $u_{1}=1, \beta=4.15, \gamma=9.11, w=8.14$. 


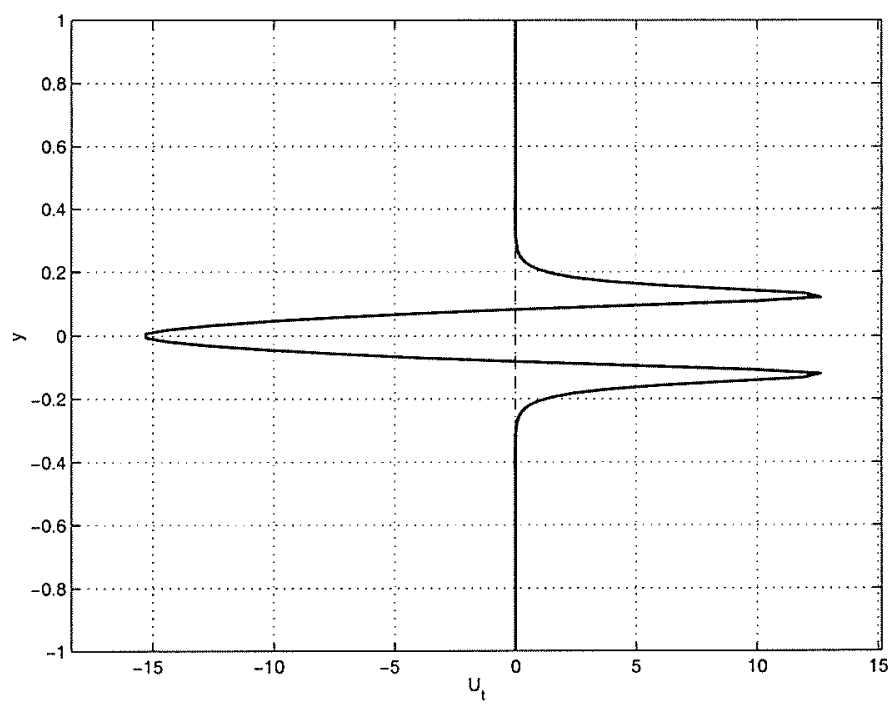

Figure 2-46: Zonal mean acceleration, $\frac{\partial \bar{u}}{\partial t}$, in a reduced-gravity model. Parameters: $u_{1}=1, \beta=4.15, \gamma=0, w=8.14$.

where $k$ is a horizontal wave number, and $c$ is the phase speed of the disturbance. Then the linearized equation of motion is given by

$$
(U(y)-c)\left(\frac{d^{2} \Phi}{d y^{2}}-k^{2} \Phi-\gamma^{2} \Phi\right)+\Phi \frac{\partial Q}{\partial y}=0,
$$

subject to no-normal-flow boundary conditions

$$
\Phi(y=\pi)=\Phi(y=-\pi)=0 .
$$

As in a two layer model, the above problem is reduced to the generalized eigenvalue problem:

$$
A \Phi=c B \Phi,
$$

where matrices $A$ and $B$ are given by (2.12) and (2.13).

Figure 2-44 shows the maximum growth rates and the real parts of the phase speed for four values of nondimensional $\gamma$, which correspond to the following values of the deformation radius: $44 \mathrm{~km}, 65 \mathrm{~km}, 100 \mathrm{~km}$ and infinite. There is substantial difference in the maximum growth rates. When the deformation radius is $44 \mathrm{~km}$, 
the flow is only weakly unstable, and there is long wave cutoff. As the deformation radius decreases, the long waves become weakly unstable. The most unstable wave increases from 256 to $285 \mathrm{~km}$ with a decrease in $\gamma$. The corresponding real parts of the phase speed are similar for various $\gamma$. As the deformation radius increases, short waves become more unstable.

The TEM analysis shows the diffusion and the rectification of the flow in the middle of the domain and the development of westward flows on the flanks of the jet when $L_{d e f}=44 \mathrm{~km}$ (Figure 2-45(a)). The diffusion of the jet is due to the eddy potential vorticity fluxes (b), whereas westward rectification is due to the residual meridional circulation (c). In the case of an infinite deformation radius, $L_{d e f}=\infty$, the reduced-gravity model becomes purely barotropic. Similar to a two layer case, the TEM analysis does not show any tendency for the formation of westward rectifications (Figure 2-46); the eddy potential vorticity fluxes have a purely diffusive effect on the mean flow.

The numerical experiments with the fully nonlinear reduced-gravity model show that the relative strength of the recirculations, defined by the ratio of the total westward to the total eastward transports, is a monotonically decreasing function of a nondimensional inverse deformation radius, $\gamma$, (Figure 2-47). When $\gamma=0$, which corresponds to an infinite dimensional deformation radius, the total westward transport is $20 \%$ of the total eastward transport, which compares well with the results of the two-layer model for a strongly barotropic basic flow. When the deformation radius was chosen to be $44 \mathrm{~km}(\gamma=9.11)$, the strength of the recirculations was negligible.

Therefore, in the case of the reduced-gravity model, the tendency for the formation of the recirculations during the stage of linear growth is misleading in that it predicts the development of recirculations for large $\gamma$ and absence of the recirculations for small $\gamma$. The initial basic flow is only slightly supercritical for large $\gamma$, and therefore there are no strongly nonlinear wave-mean and wave-wave interactions which would 


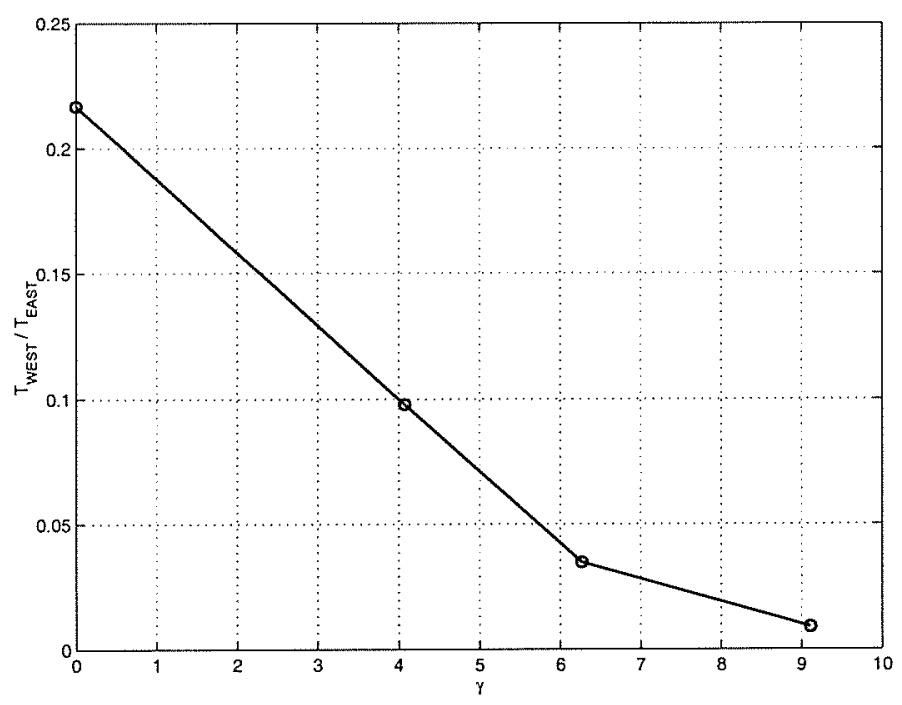

Figure 2-47: The ratio of westward to eastward transport in the equilibrated jet vs nondimensional $\gamma$ in a reduced-gravity model. Parameters: $u_{1}=1, \beta=4.15, w=$ 8.14.

be necessary for the development of the recirculations by barotropic instability. In the case of large $\gamma$, the basic flow is strongly supercritical, and hence the nonlinear eddy field is strong enough to produce westward rectifications.

\subsection{Conclusions}

The formation of westward recirculations is studied in the model of a free nonlinear spin down of zonally symmetric, quasi-geostrophic, unstable jets. The model (Flierl, personal communication) has weak sponge layers and a numerical filter, which imply only weak dissipation of energy and enstrophy.

The recirculations are robust features of a two-layer model, which includes the mechanisms of both baroclinic and barotropic instabilities. The relative strength of the recirculations, defined as the ratio of the total westward to the total eastward transport, is a function of the nondimensional parameters of the model. It decreases as the value of nondimensional $\beta$ increases. This decrease in the recirculation strengths 
is due to a decrease in the zone of homogenized potential vorticity, which in turn is due to (approximate) enstrophy conservation.

When $\beta, \gamma$ and total energy level are fixed, the strength of the recirculations is maximum for strongly baroclinic flows and minimum for intermediate values of a baroclinic parameter, $\frac{u_{1}-u_{2}}{F_{1} u_{2}+F_{2} u_{1}}$, where $u_{i}$ is $i-$ th layer nondimensional velocity amplitude and $F_{i}$ is $i$-th layer Froude number. In strongly baroclinic cases, eddies marginally equilibrate the flow and create a wide zone of homogenized potential vorticity in the lower layer. This result is in agreement with the study of a weakly-nonlinear equilibration of Phillips' model with weak dissipation (Pedlosky [28]). In the upper layer, a positive potential vorticity gradient on the axis of the jet decreases by less than $10 \%$, thus appearing as an efficient barrier to mixing. Most vigorous mixing occurs on the edges of the jet in the upper layer and under the axis of the jet in the lower layer.

As the jet becomes more barotropic, a positive potential vorticity gradient develops on the axis of the jet in the lower layer, reflecting a more stable flow structure. In strongly barotropic jets, there is vigorous mixing both across the positive potential vorticity gradient and on the edges of the jet, leading to a decrease on a values of the upper layer potential vorticity gradient on the axis of the jet by more than $45 \%$.

The linear stability analysis applied to a two-layer jets shows that in strongly baroclinic cases, the lower layer recirculations are driven by the eddy heat fluxes through the residual meridional circulation. In the upper layer, the divergence of eddy potential vorticity fluxes tends to simply "diffuse" the jet during the stage of linear growth. Nonlinear eddy-eddy and eddy-mean flow interactions are further important in inducing the upper layer recirculations. The linear analysis shows that the strength of the residual meridional circulations increases with an increase in the width of basic flow. That is why a "mean flow" model (Flierl, personal communication) successfully reproduces recirculations when applied to strongly baroclinic basic flows.

For strongly barotropic two-layer basic flows, which are strongly supercritical, the linear eddy potential vorticity fluxes tend to diffuse the jet in both layers without 
any tendency for the formation of westward flows on the flanks. As the basic flow becomes wider, it stabilizes. Therefore, strongly nonlinear regimes, which allow for both eddy-mean flow and eddy-eddy interactions, are required for the emergence of recirculations for strongly barotropic basic flows.

Thus, although the recirculations develop in a two-layer model for barotropic and baroclinic flows, processes leading to their generation are different. In baroclinic case, eddy-mean flow interaction is powerful enough to drive recirculations. In barotropic cases, strongly nonlinear eddy-mean flow and eddy-eddy interactions are required for the recirculations development. The existence of two different mechanisms is responsible for a non-monotonic dependence of the recirculation strength on the baroclinic parameter on nonlinear experiments.

In the reduced-gravity model, which has just the mechanism of barotropic instability, recirculations form as a result of a nonlinear spin down of an unstable basic flow only for the case of an infinite dimensional deformation radius. For deformation radii smaller than $65 \mathrm{~km}$, the total westward transport is less than $5 \%$ of the total eastward transport.

The results of the linear stability analysis are misleading since they imply a weak tendency for the formation of the recirculations for small dimensional deformation radii and the absence of the recirculations for an infinite dimensional deformation radius. The linear stability analysis is not helpful since the basic flow is only slightly supercritical for large $\gamma$, implying a weak eddy field. 


\section{Chapter 3}

\section{Colliding Jets Model Formulation}

\subsection{Introduction}

In the following Chapters, we investigate the problem of low frequency variability and associated multiple dynamical regimes resulting from the internal dynamics of an unstable quasi-geostrophic jet and its recirculations. Particularly, we raise the following questions: Are low frequency variability and multiple dynamical regimes an inherent part of the internal dynamics of a quasi-geostrophic jet and its recirculations? Are there any differences between barotropic and baroclinic instabilities as mechanisms affecting low frequency variability? Which dynamical regimes are associated with low frequency variability and which precesses are responsible for transitions between them? Are there differences between low frequency variability and multiple dynamical regimes in the globally forced (wind-driven) models and boundary-forced models, which we use in a present study?

To study the above questions, we designed regional reduced-gravity and two layer quasi-geostrophic models that are forced by prescribed inflow and outflow boundary conditions; dissipation is the only active forcing term in the equations of motion. The open boundaries represent the border between the "outside" region of slow Sverdrup interior, where the wind forcing plays a crucial role, and the "interior" region 
of the western boundary current, its eastward extension and its recirculations, where the internal dynamics of the jet is dominant. In our model, the role of wind-forcing is limited to defining a particular distribution of potential vorticity and streamfunction along the inflow and outflow boundaries, a distribution that is fixed in time. Notice that, in the wind forced models which exhibit multiple dynamical regimes, the inflow into the jet can change, since Sverdrup balance is not exact; such changes may influence the dynamical behavior.

\subsection{Model Formulation}

\subsubsection{Governing Equations}

The numerical model is a discretized version of the time-dependent two-layer quasigeostrophic potential vorticity equations on a $\beta$ - plane:

$$
\frac{\partial q_{i}}{\partial t}+\mathcal{J}\left(\psi_{i}, q_{i}\right)=\mathcal{D}_{i}+\mathcal{S}_{i} \quad \text { in } \Omega
$$

subject to the boundary conditions:

$$
\psi_{i}=\Psi_{i}, \quad \nabla^{2} \psi_{i}=\mathcal{L}_{i}, \quad \nabla^{4} \psi_{i}=\mathcal{M}_{i} \quad \text { in } \bar{\Omega}
$$

Here, $\psi_{i}=\psi_{i}(x, y)$ is a quasi-geostrophic streamfunction, $q_{i}=q_{i}(x, y)=\nabla^{2} \psi_{i}+\beta y+$ $F_{i}\left(\psi_{3-i}-\psi_{i}\right)$ is potential vorticity, and $i=1,2$ is a layer index. The rectangular model domain is defined by $\Omega \cup \bar{\Omega}$, where $\Omega$ is the set of all the internal points and $\bar{\Omega}$ is the set of all the boundary points. On the boundaries, the streamfunction, its Laplacian and the square of the Laplacian are prescribed as known functions $\Psi_{i}, \mathcal{L}_{i}$ and $\mathcal{M}_{i}$. The dissipation is provided by biharmonic diffusion and by bottom or interfacial (in the case of the reduced-gravity formulation) friction, so that $\mathcal{D}_{1}=-\mathcal{A}_{6} \nabla^{6} \psi_{1}$ and $\mathcal{D}_{2}=-\mathcal{A}_{2} \nabla^{2} \psi_{2}-\mathcal{A}_{6} \nabla^{6} \psi_{2}$. In addition, the dissipation $\mathcal{S}_{i}$ in the sponge layers, of which details are presented later, prevents part of the outgoing radiation from reflecting back onto the interior domain. 


\subsubsection{Dimensional Scales}

The dimensional scales are chosen as follows. The horizontal length scale is $L=$ $2400 \mathrm{~km}$, the advective velocity scale is $U=1 \mathrm{~m} \mathrm{sec}^{-1}$, which implies an advective time scale $T=L / U=27.8$ days. The layer thicknesses in the reference run are $D_{1}=600 \mathrm{~m}$ and $D_{2}=4 \mathrm{~km}$. The Coriolis parameter is $f_{0}=7.25 \cdot 10^{-5} \mathrm{sec}^{-1}$, its meridional gradient is $\beta^{\text {dim }}=1.97 \cdot 10^{-11} \mathrm{~m}^{-1} \mathrm{sec}^{-1}$. The baroclinic deformation radius is chosen as $L_{\text {def }}=47 \mathrm{~km}$. Reduced gravity is $g^{\prime}=0.02 \mathrm{~m} \mathrm{sec}^{-2}$. The dimensional biharmonic diffusion coefficient, $\mathcal{A}_{6}^{\text {dim }}$, equals either $8 \cdot 10^{10} \mathrm{~m}^{4} \mathrm{sec}^{-1}$ or $8 \cdot 10^{8} \mathrm{~m}^{4} \mathrm{sec}^{-1}$. The values of the dimensional bottom friction or interfacial friction, $\mathcal{A}_{2}^{\text {dim }}$, vary.

\subsubsection{Model Domain}

The model domain is a rectangular basin defined as

$$
\Omega \cup \bar{\Omega}=\left\{(x, y) \mid x_{w} \leq x \leq x_{e}, y_{s} \leq y \leq y_{n}\right\}
$$

where $x_{e}=-x_{w}=L_{x} / 2 L_{y}, y_{n}=-y_{s}=0.5$. The dimensional west-east and southnorth extents of the domain are $L_{x}=4667 \mathrm{~km}$ and $L_{y}=2400 \mathrm{~km}$. The model has a uniform grid with 257 by 129 grid points, which implies a horizontal resolution of 18.5 $\mathrm{km}$. High resolution runs with 513 by 257 grid points and a corresponding horizontal resolution of $\Delta x=9.12 \mathrm{~km}$ and $\Delta y=9.38 \mathrm{~km}$ were performed to ensure that the model's behavior is robust.

\subsubsection{Nondimensional Parameters}

The model has the following nondimensional parameters:

- the nondimensional beta, defined as the ratio of the advective time scale to the barotropic Rossby wave period: $\beta=\beta^{\operatorname{dim}} L^{2} / U$; 
- the inverse deformation radius, defined as the ratio of a horizontal length scale to the internal baroclinic deformation radius: $\gamma=\sqrt{F_{1}+F_{2}}=L / L_{\text {def }}$, where the $i$-th layer Froude number is given by $F_{i}=\frac{f_{0}^{2} L^{2}}{g^{\prime} D_{i}}$

- the depth ratio: $\delta=D_{1} / D_{2}$;

- the ratio of the maximum of the lower layer inflow streamfunction, $\Psi_{2}$, to the maximum of the upper layer inflow streamfunction, $\Psi_{1}: \alpha=\max \left(\Psi_{2}\right) / \max \left(\Psi_{1}\right)$,

- the nondimensional interfacial or bottom friction coefficient, which is the ratio of the advective time scale to the frictional decay time scale: $\mathcal{A}_{2}=\mathcal{A}_{2}^{\text {dim }} L / U$,

- the nondimensional biharmonic diffusion coefficient, which is the ratio of the advective time scale to the biharmonic diffusion time scale: $\mathcal{A}_{6}=\mathcal{A}_{6}^{d i m} L^{-3} U^{-1}$.

\subsubsection{Forcing and Boundary Conditions}

The model is forced by the set of prescribed inflow and outflow boundary conditions. Such a forcing isolates baroclinic and barotropic instabilities as mechanisms responsible for low-frequency variability and multiple dynamical regimes.

Two thin jets of equal strength enter the domain from the south and the north (Figure 3-1), flow along the closed western boundary, and collide in the middle of the domain, thereby creating an eastward jet. The latter propagates across the domain and leaves through the opened eastern boundary. The other two regions of inflow are located in the northern and southern parts of the eastern boundary, where the entering flow has weak westward zonal velocity. The geometry of the model allows for the interaction between the western boundary current and the recirculations generated by the instabilities of the eastward jet. 


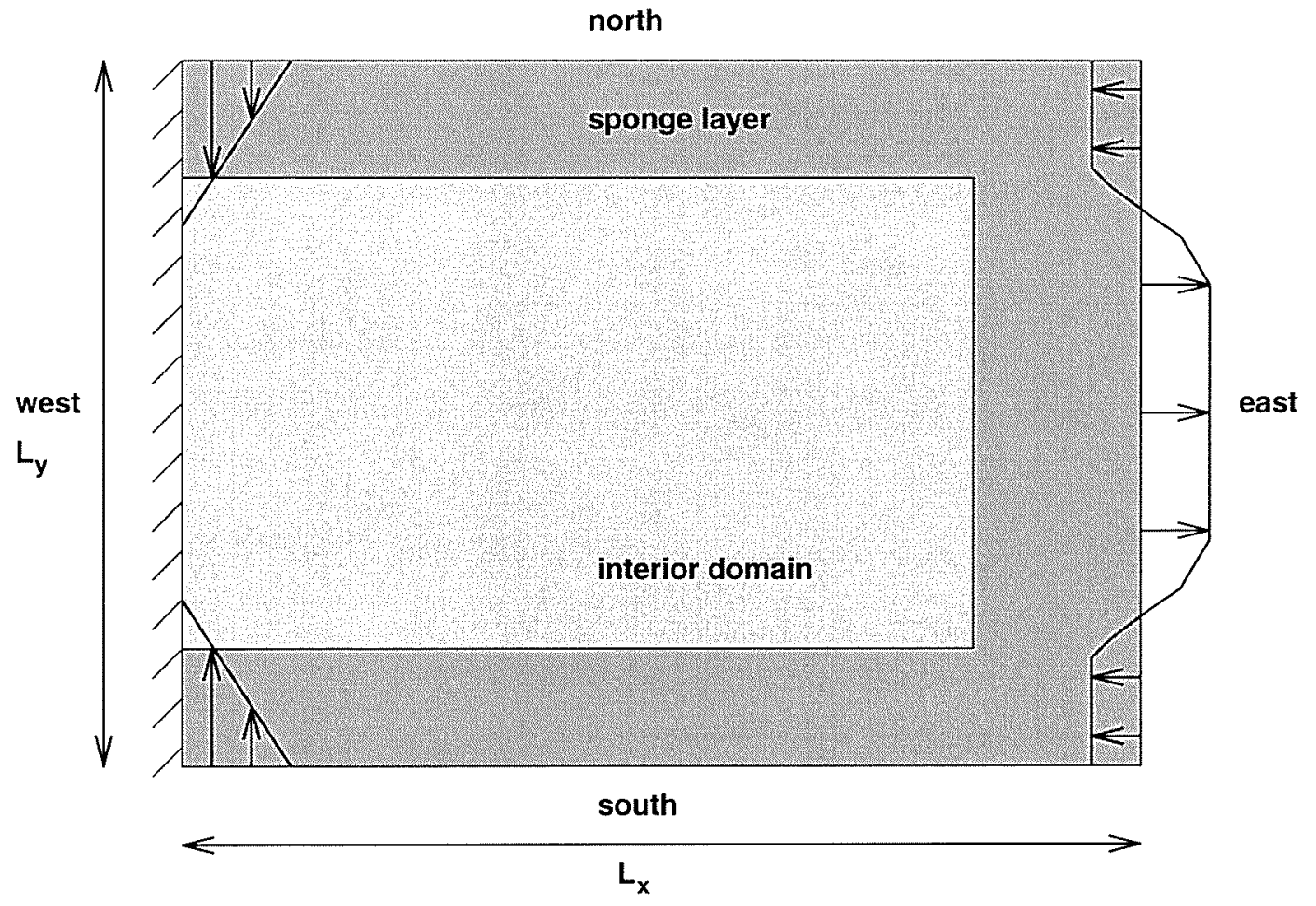

Figure 3-1: The geometry of the colliding jets model.

The inflow and the outflow on the open boundaries are implemented using a given streamfunction field, which is kept fixed in time:

$$
\begin{aligned}
& \psi_{i}(x, y)=R_{i} \begin{cases}0 & \text { for }\left\{(x, y) \mid x=x_{w}, y_{s} \leq y \leq y_{n}\right\} \\
\Psi_{i}^{\text {east }} & \text { for }\left\{(x, y) \mid x=x_{e}, y_{s} \leq y \leq y_{n}\right\} \\
\Psi_{i}^{\text {south }} & \text { for }\left\{(x, y) \mid x_{w} \leq x \leq x_{e}, y=y_{s}\right\} \\
-\Psi_{i}^{\text {south }} & \text { for }\left\{(x, y) \mid x_{w} \leq x \leq x_{e}, y=y_{n}\right\}\end{cases} \\
& \nabla^{(k)} \psi_{i}(x, y)=R_{i} \begin{cases}0 & \text { for }\left\{(x, y) \mid x=x_{w}, y_{s} \leq y \leq y_{n}\right\}, \\
\frac{\partial^{(k)}}{\partial y^{(k)}} \Psi_{i}^{\text {east }} & \text { for }\left\{(x, y) \mid x=x_{e}, y_{s} \leq y \leq y_{n}\right\} \\
\frac{\partial^{(k)}}{\partial x^{(k)}} \Psi_{i}^{\text {south }} & \text { for }\left\{(x, y) \mid x_{w} \leq x \leq x_{e}, y=y_{s}\right\} \\
-\frac{\partial^{(k)}}{\partial x^{(k)}} \Psi_{i}^{\text {south }} & \text { for }\left\{(x, y) \mid x_{w} \leq x \leq x_{e}, y=y_{n}\right\},\end{cases}
\end{aligned}
$$

where $k=2,4, R_{1}=1$ and $R_{2}=\alpha$. The coefficient $\alpha$ varies between zero and one, thus allowing the creation of inflow/outflow conditions with different degrees of baroclinicity. 
We chose a free-slip boundary condition for the closed western boundary. Berloff and McWilliams [3] compared the free- and no-slip boundary conditions in a framework of a three-layer wind-driven quasi-geostrophic model with lateral diffusion and showed that the main difference in the resulting dynamics was that for computationally achievable frictional parameters, the western boundary current did not have local instability with a free-slip boundary condition.

Here, the boundary conditions are chosen in such a way that the geostrophic transport into the domain balances the geostrophic transport out of the domain. However, the total mass, which also includes ageostrophic fields, is not fixed in time. The presence of open boundaries allows for small fluctuations of mass due to variations in the order of Rossby number fields. The description of such fluctuations is presented in Chapter 4.

There is zero potential vorticity flux through the boundaries:

$$
\oint_{\bar{\Omega}} q_{i} \mathbf{u}_{\mathbf{i}} \cdot \mathbf{n} d \bar{\Omega}=0
$$

where $\mathbf{u}_{\mathbf{i}}=\left(u_{i}, v_{i}\right)^{T}=\left(-\frac{\partial \psi_{i}}{\partial y}, \frac{\partial \psi_{i}}{\partial x}\right)^{T}$ is a quasi-geostrophic velocity vector and $\mathbf{n}$ is a vector normal to the boundary $\bar{\Omega}$. The contribution from planetary vorticity, $\beta y$, is zero because the open boundaries are symmetric: $y_{n}=-y_{s}$. The potential vorticity, $\nabla^{2} \psi_{i}+F_{i}\left(\psi_{3-i}-\psi_{i}\right)$, also integrates to zero since the streamfunction on the northern boundary is antisymmetric to the streamfunction on the southern boundary.

However, the potential vorticity flux into the southern (northern) part of the domain depends on the particular form of the inflow and outflow conditions. Different conditions may result in an intensified or a weakened potential vorticity exchange across the potential vorticity front associated with the eastward jet.

\subsubsection{Sponge Operator}

Traditionally, models with open boundaries use so-called radiation condition schemes on the outflow boundaries. Such radiation conditions attempt to allow the outgoing 
part of the disturbance to propagate out of the domain without reflecting back and therefore without contaminating the interior solution.

However, radiation conditions were not used in the present model for the following reasons. The open eastern boundary in the model has regions of both inflow and outflow (Figure 3-1). A radiation condition can be applied only on the outflow part of the boundary; the inflow is fixed in time, thus providing steady forcing. Naturally, the information on the outgoing disturbance that comes from the interior of the domain and leaves through the outflow part of the boundary is independent from the information that enters the domain from "outside" through the inflow part of the boundary. This results in a discontinuity between the inflow and outflow values of the field to which a radiation condition is applied (here, the streamfunction field).

As a result, in our model, the problem of outgoing radiation is resolved by combining prescribed inflow/outflow boundary conditions with an absorbing sponge layer, which is represented in Equation (3.1) as

$$
\mathcal{S}_{i}=-S(x, y)\left(q_{i}-Q_{i}\right)-\frac{\partial S}{\partial x}\left(\frac{\partial \psi_{i}}{\partial x}-\frac{\partial \Psi_{i}}{\partial x}\right)-\frac{\partial S}{\partial y}\left(\frac{\partial \psi_{i}}{\partial y}-\frac{\partial \Psi_{i}}{\partial y}\right)
$$

where $S(x, y)$ is a sponge function, $q_{i}$ and $\psi_{i}$ are instantaneous potential vorticity and streamfunction fields, and $Q_{i}$ and $\Psi_{i}$ are prescribed "relaxation" potential vorticity and streamfunction fields. The latter are chosen as initial fields. Therefore, in addition to absorbing the outgoing radiation, the sponge layer also allows for a smooth transition of the instantaneous interior flow to the prescribed distribution of inflow and outflow boundary conditions.

A particular form of the sponge function is given by

$$
S(x, y)=\frac{L}{U T_{\text {decay }}}\left\{\begin{array}{cc}
0 & \text { for } x<a,-b<y<b, \\
\left(\frac{x-a}{W_{\text {east }}}\right)^{2}+\left(\frac{y-b}{W_{\text {north }}}\right)^{2} & \text { for } x>a, y>b, \\
\left(\frac{x-a}{W_{\text {east }}}\right)^{2}+\left(\frac{y+b}{W_{\text {south }}}\right)^{2} & \text { for } x>a, y<-b,
\end{array}\right.
$$

where $T_{\text {decay }}=24$ hours is the minimum decay scale in the sponge layer, $a=x_{e}-30 \Delta x$ and $b=y_{n}-25 \Delta y$. The corresponding dimensional width of the sponge layer in the 
(a)

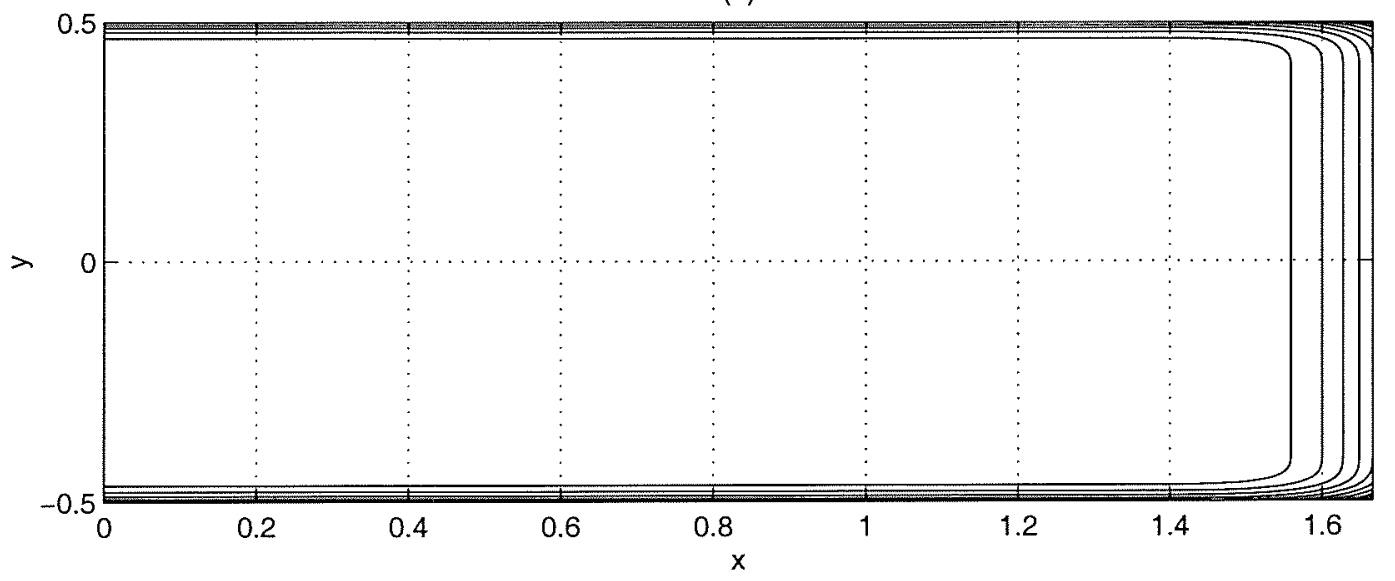

(b)

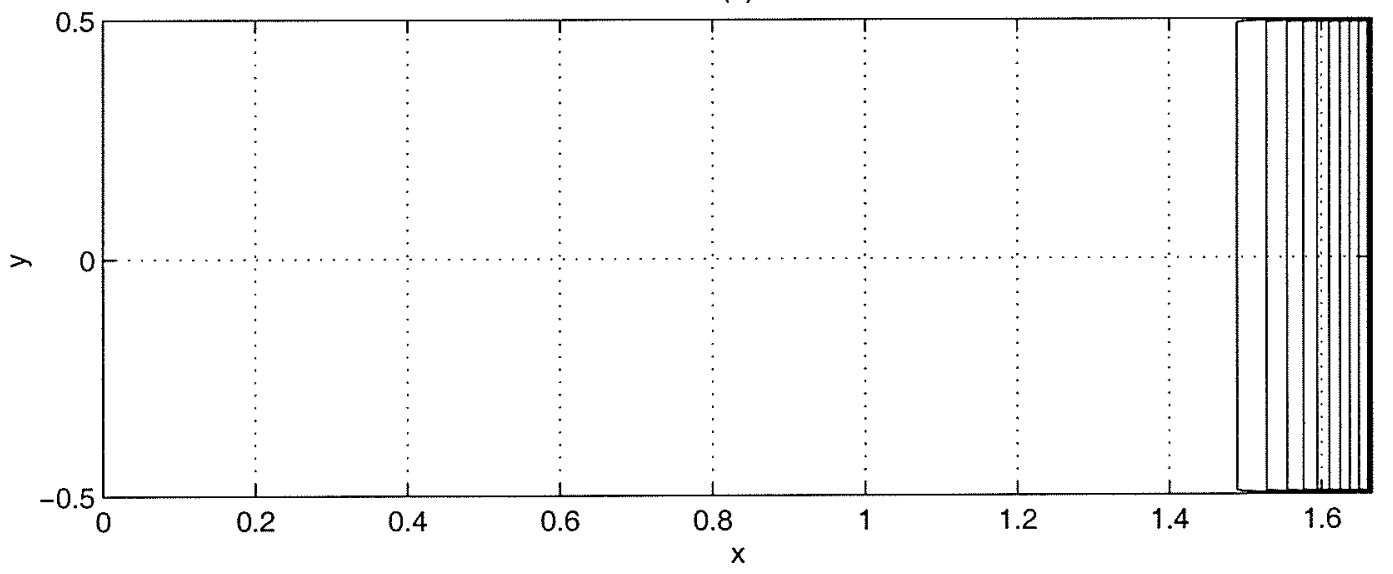

(c)

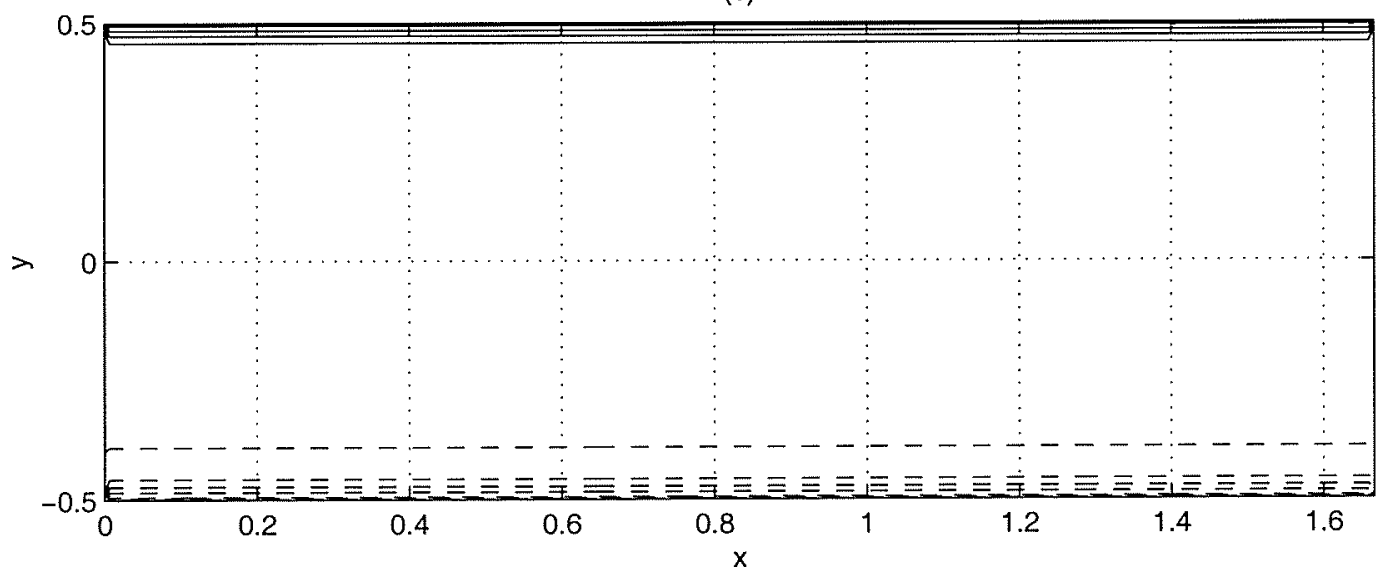

Figure 3-2: Contours of sponge function, $S(x, y),(\mathrm{a})$ and its derivatives, $\frac{\partial S}{\partial x}$ (b) and $\frac{\partial S}{\partial y}(\mathrm{c})$. Solid line is used for positive values, dashed line is used for negative values. 
eastern part of the domain is $W_{\text {east }}=550 \mathrm{~km}$ and the width in the northern and southern parts of the domain is $W_{\text {north }}=W_{\text {south }}=470 \mathrm{~km}$ (Figure 3-2).

\subsubsection{Numerical scheme}

The governing equations are discretized in time with a centered leapfrog scheme to integrate forward the potential vorticity field:

$$
q_{k, l}^{t+1}=q_{k, l}^{t-1}+2 \Delta t\left(\mathcal{J}_{k, l}^{t}+\mathcal{S}_{k, l}^{t-1}-\mathcal{D}_{k, l}^{t-1}\right)
$$

where $k$ and $l$ denote grid points in $x$ and $y$ directions, $t-1, t$, and $t+1$ are

consecutive time steps, $\mathcal{J}_{k, l}^{t}$ is a Jacobian term, $\mathcal{S}_{k, l}^{t-1}$ is a sponge function term, and $\mathcal{D}_{k, l}^{t-1}$ is a dissipation term. To suppress a computational mode associated with the leapfrog scheme, the potential vorticity fields at $t+1$ and $t-1$ are averaged every 20 iterations to get a corrected potential vorticity field at $t+1$.

To calculate $\mathcal{J}_{k, l}^{t}$, the Arakawa Jacobian formulation is used. This allows no net advection out of the interior region of potential vorticity, enstrophy or energy as long as the streamfunction is constant along the boundary.

\subsubsection{The Inversion of the Potential Vorticity Field}

The time stepping scheme is applied to the potential vorticity field which is then inverted to get the streamfunction. The inversion problems are solved separately for the barotropic, $\psi_{b t}=F_{1} \psi_{2}+F_{2} \psi_{1}$, and baroclinic, $\psi_{b c}=\psi_{2}-\psi_{1}$, modes and are given by

$$
\begin{array}{r}
\nabla^{2} \psi_{b t}=F_{1} q_{2}+F_{2} q_{1}-\beta \gamma^{2} y \text { in } \Omega \\
\psi_{b t}=F_{1} \psi_{2}+F_{2} \psi_{1} \quad \text { in } \bar{\Omega}
\end{array}
$$

and

$$
\begin{aligned}
\left(\nabla^{2}-\gamma^{2}\right) \psi_{b c} & =q_{2}-q_{1} \quad \text { in } \Omega \\
\psi_{b c} & =\psi_{2}-\psi_{1} \quad \text { in } \bar{\Omega}
\end{aligned}
$$


The upper and lower layer streamfunctions are then restored:

$$
\psi_{i}=\left(\psi_{b t}+(-1)^{i} F_{i} \psi_{b c}\right) \gamma^{-2}
$$

FORTRAN 77 routines, H2GCIS and H2GCSS, from the CRAYFISH package were used for solving the Helmholtz Equations (3.10) and (3.11).

\subsubsection{Initialization of the Model}

To ensure the stability of currents entering the domain through the open boundaries, the model is initialized with a streamfunction field constructed from the Fofonoff solution. The latter satisfies Arnold's sufficient condition for stability of a stationary nonparallel flow [2], since $\frac{\partial q_{i}}{\partial \psi_{i}}>0$.

To create an initial condition, we first define the following streamfunction field (Figure 3-3a):

$$
\psi_{*}= \begin{cases}-\psi_{F} & \text { for } x_{w}^{*} \leq x \leq x_{e}^{*}, 0 \leq y \leq y_{n} \\ \psi_{F} & \text { for } x_{w}^{*} \leq x \leq x_{e}^{*}, y_{s} \leq y \leq 0\end{cases}
$$

where $x_{e}^{*}=-x_{w}^{*}=L_{x}^{*} / 2 L_{y}, L_{x}^{*}=5000 \mathrm{~km}$ and $\psi_{F}$ is the Fofonoff solution satisfying $\psi_{F}=\frac{\beta y_{0}}{k^{2}}\left\{\frac{\left(y+y_{0}\right)}{y_{0}}-\frac{\sinh k\left(y+y_{0}\right)}{\sinh k y_{0}}+\frac{2}{\pi} \sum_{n=1}^{\infty} \frac{(-1)^{n}}{n}\left(\frac{k}{k_{n}}\right)^{2} \frac{\cosh k_{n} x}{\cosh k_{n} x_{0}} \sin \frac{n \pi\left(y+y_{0}\right)}{y_{0}}\right\}$, where $y_{0}=x_{0}=L_{x}^{*} / 2 L_{y}, k_{n}^{2}=k^{2}+\left(n \pi / y_{0}\right)^{2}, k^{2}=\gamma^{2}+C, C \geq 0$.

The potential vorticity field, $q_{*}=\beta y+\nabla^{2} \psi_{*}-\gamma^{2} \psi_{*}$, has a discontinuity in the middle of the domain (Figure 3-4), since

$$
\lim _{y \rightarrow 0^{-}} q(x, y)=-\lim _{y \rightarrow 0^{+}} q(x, y) \neq 0 .
$$

The discontinuity may produce instability, and therefore we smooth it out by interpolating $q_{*}$ between its values at $y=-0.1$ and $y=0.1$ to get a new potential vorticity field, $q_{* *}$ (Figure 3-3d). The inversion of this field results in a streamfunction, $\psi_{* *}$, (Figure 3-3c) that corresponds to a $600-\mathrm{km}$ wide jet. The initial condition thus is 
(a)

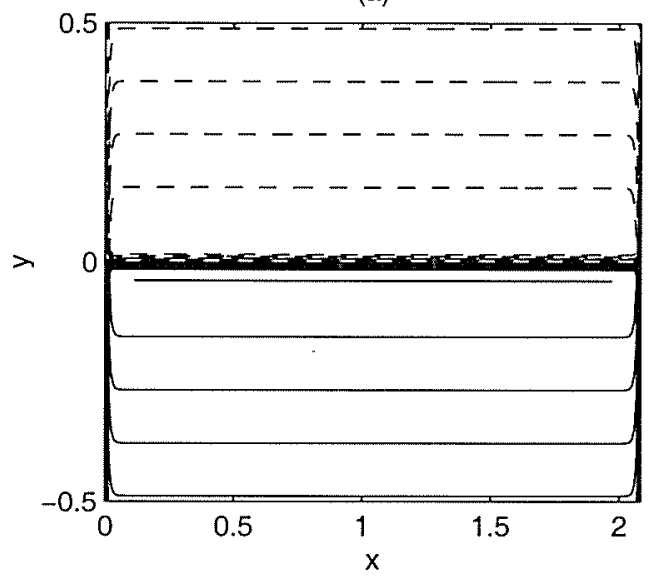

(c)

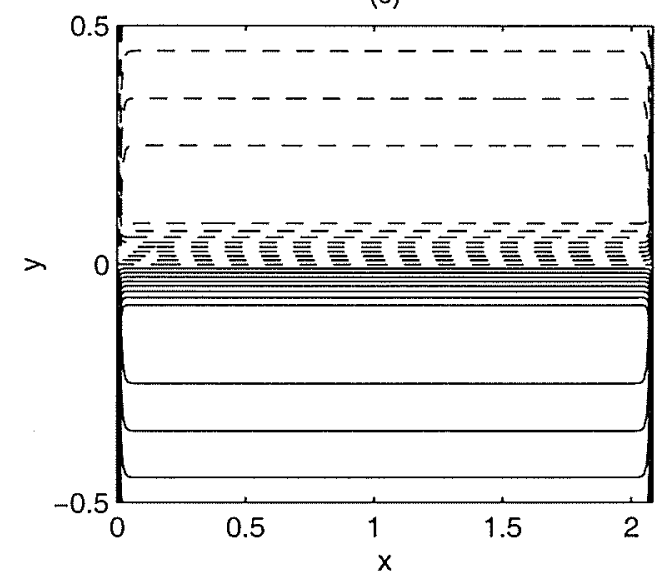

(e)

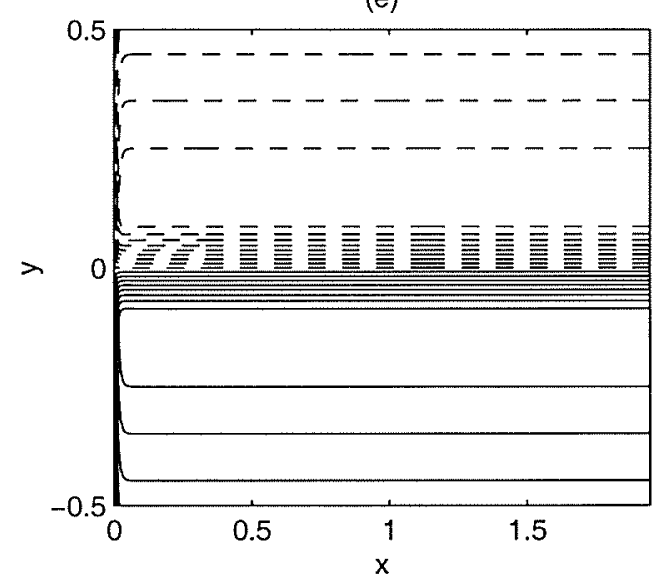

(b)

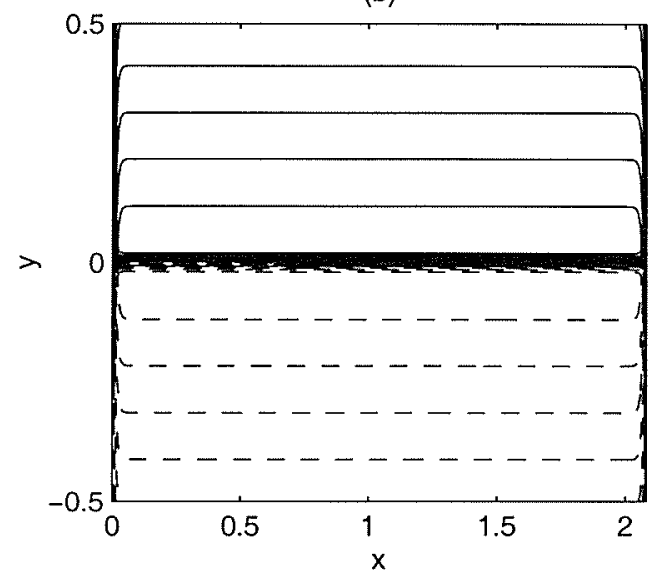

(d)

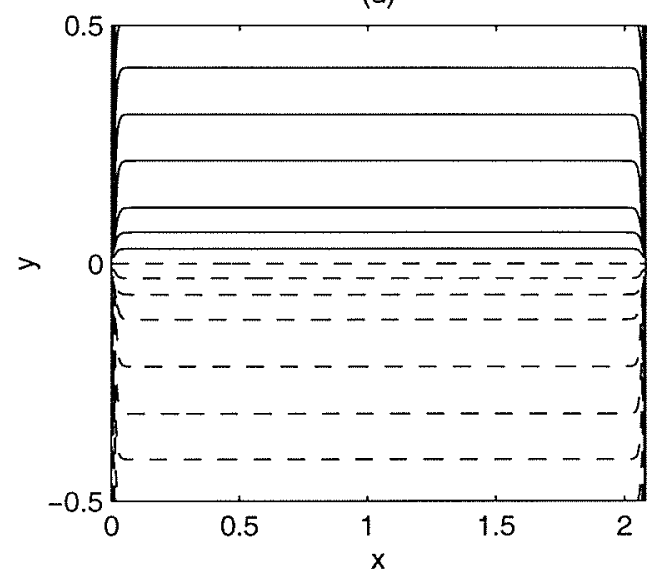

(f)

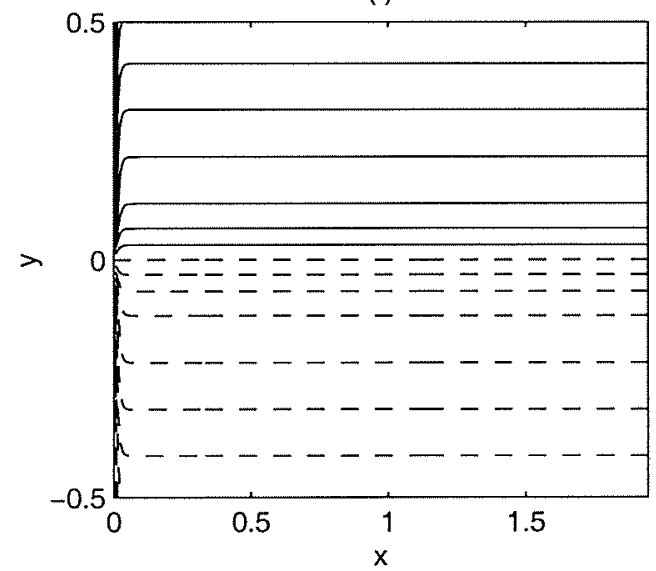

Figure 3-3: Contours of streamfunction $\psi_{*}$ (eq. 3.13) (a) and of corresponding potential vorticity $q_{*}$ (b). Contours of streamfunction $\psi_{* *}$ (c) and potential vorticity $q_{* *}$ (d). Contours of initial streamfunction (e) and potential vorticity. Solid line is used for positive values, dashed line is used for negative values. 


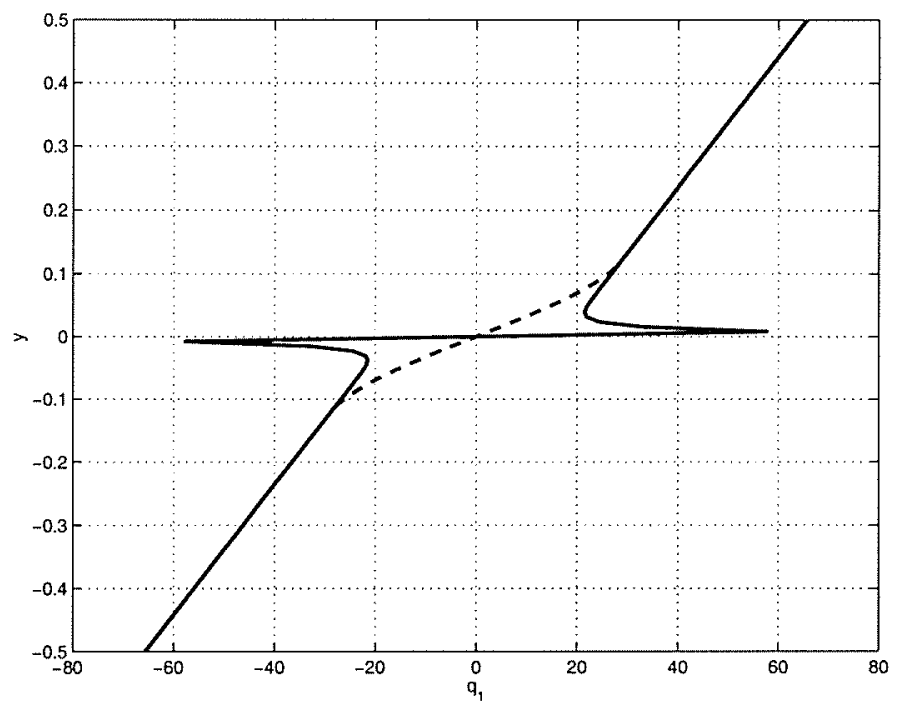

Figure 3-4: Solid line: the meridional profile of potential vorticity, $q_{*}=\beta y+\nabla^{2} \psi_{*}$ $\gamma^{2} \psi_{*}$, where $\psi_{*}$ is given in Equation 3.13. Dashed line: the meridional profile of interpolated potential vorticity, $q_{* *}$.

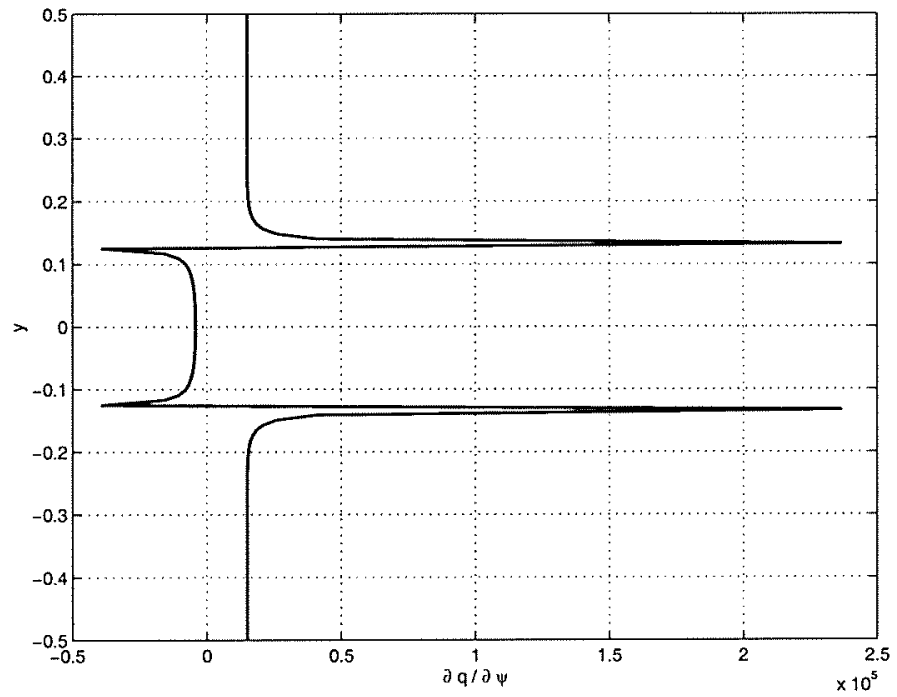

Figure 3-5: $\frac{\partial q_{* *}}{\partial \psi_{* *}}$ in the middle of the domain. 
chosen as $\psi=\psi_{* *}$ for $(x, y) \in \bar{\Omega}$ (Figure 3-3 e,f). Notice that after the interpolation, the necessary condition for instability is satisfied, since there are regions where $\frac{\partial q_{* *}}{\partial \psi_{* *}}$ is negative (Figure 3-5). Smoothed field is no longer a steady solution.

\subsection{Energetics of the Model}

\subsubsection{Energy Equation}

Multiplying the upper and the lower layer potential vorticity equations (3.1) by $-\psi_{1} \frac{D_{1}}{D}$ and $-\psi_{2} \frac{D_{2}}{D}$ correspondingly and adding them together give the following energy equation:

$$
\frac{\partial E}{\partial t}+\nabla \cdot \mathbf{P}=G
$$

Here, $E$ is the total energy defined by the sum of the depth-integrated upper and lower layer kinetic energy and the available potential energy:

$$
E=\sum_{i=1}^{2} \frac{d_{i}}{2}\left\{\left(\frac{\partial \psi_{i}}{\partial x}\right)^{2}+\left(\frac{\partial \psi_{i}}{\partial y}\right)^{2}\right\}+\frac{F_{0}}{2}\left(\psi_{1}-\psi_{2}\right)^{2}
$$

where $F_{0}=\frac{F_{1} d_{1}+F_{2} d_{2}}{2}, d_{i}=\frac{D_{i}}{D}, i=1,2$. The $x$ - and $y$-components of the energy flux vector, $\mathbf{P}=P_{(x)} \mathbf{i}+P_{(y)} \mathbf{j}$, are defined by

$$
P_{(x)}=\sum_{i=1}^{2}-d_{i} \psi_{i}\left\{\frac{\partial v_{i}}{\partial t}+\Pi_{i} u_{i}+\frac{\beta}{2} \psi_{i}\right\}
$$

and

$$
P_{(y)}=\sum_{i=1}^{2} d_{i} \psi_{i}\left\{\frac{\partial u_{i}}{\partial t}-\Pi_{i} v_{i}\right\}
$$

where $\Pi_{i}=q_{i}-\beta y$. The dissipation term is given by

$$
G=-\sum_{i=1}^{2} d_{i} \psi_{i}\left(\mathcal{D}_{i}+\mathcal{S}_{i}\right)
$$

where $\mathcal{D}_{i}$ and $\mathcal{S}_{i}$ are defined in section 3.2.1. In the absence of dissipation, the time changes of the total energy are only due to the divergence of the energy flux vector. 


\subsubsection{Energy Sources and Sinks}

Total energy sources and sinks can be identified by integrating equation (3.14) over the model domain:

$$
\begin{array}{r}
\frac{\partial \mathbf{E}}{\partial t}=-\int_{x_{w}}^{x_{e}}\left(P_{(y)}\left(x, y_{n}\right)-P_{(y)}\left(x, y_{s}\right)\right) d x-\int_{y_{s}}^{y_{n}} P_{(x)}\left(x_{e}, y\right) d y+ \\
\int_{x_{w}}^{x_{e}} \int_{y_{s}}^{y_{n}} G(x, y) d x d y
\end{array}
$$

where $\mathrm{E}=\int_{x_{w}}^{x_{e}} \int_{y_{s}}^{y_{n}} E d x d y$.

The first two integrals on the right-hand side of equation (3.19) are energy sources provided by inflows through the open boundaries, whereas the last integral is the energy sink due to dissipation. It is important to recognize that the time dependence of the energy source integrals stems from the following terms: $\psi_{i}\left(x_{e}, y\right) \frac{\partial}{\partial t} v_{i}\left(x_{e}, y\right), \psi_{i}\left(x, y_{n}\right) \frac{\partial}{\partial t} u_{i}\left(x, y_{n}\right)$ and $\psi_{i}\left(x, y_{s}\right) \frac{\partial}{\partial t} u_{i}\left(x, y_{s}\right)$. Due to the presence of a strong sponge layer, which acts to damp perturbations from the prescribed field, $\Psi_{i}$, the acceleration terms are negligible. Therefore, the energy input due to inflow/outflow boundary conditions is time-independent, which implies that time variations of total energy are only due to correlation between the solution and the dissipation term.

In contrast, in the wind-driven quasi-geostrophic models, even though the wind-stress curl is steady, the energy input, defined as the correlation between the streamfunction and the wind-stress curl, $-\int_{x} \int_{y} \psi \cdot \nabla \times \tau d x d y$, can vary in time, depending on the solution structure. Primeau [29] showed a remarkable similarity between the steady and the time-dependent solutions of a wind-driven reduced-gravity model of McCalpin and Haidvogel [25] and estimated the differences in the energy input for multiple equilibria at 13\%. Scott and Straub [34] found that antisymmetric steady solutions with strong jet penetration and the jet axis aligned with the maximum wind stress were the most efficient at maximizing the energy input. Non- 
symmetric steady solutions, on the other hand, were less correlated with the wind forcing and thus provided smaller energy input.

In their time-dependent model, McCalpin and Haidvogel [25] found that the symmetry of the global wind forcing structure was important for maintaining the high energy dynamical regimes, which closely resembled the antisymmetric steady state solutions of Primeau [29]. For high values of the wind forcing asymmetry parameter, the high energy state disappeared, and the flow was characterized by the low energy state with weakly penetrating jet and strong meanders.

Consider the following thought experiment illustrating the importance of global wind forcing for the existence and maintenance of multiple dynamical regimes. Assuming all parameters and the basic state are the same in the wind-driven and boundary-forced models, the linear vicinities of a fixed point corresponding to the antisymmetric solution should be topologically equivalent for the two models, since the wind-forcing drops out of the linear equations of motion. As we show later, for the antisymmetric steady solutions, the structure and the growth rate of the most unstable modes are indeed similar for the regional and wind-driven reduced-gravity models. Now, suppose the wind-driven model trajectory is recovering from a low energy state and is moving towards a high energy state, thus approaching a linear neighborhood of an antisymmetric fixed point. In this case, the recirculations become larger, and the jet straightens. The spatial structure of the solution is now strongly correlated with the antisymmetric wind stress curl, and thus the energy input is increased. Therefore, if the model trajectory is close to the fixed point, but the nonlinear interactions are still at work, the wind forcing could play a crucial role in further "directing" the model towards a steady antisymmetric solution. The dissipation is also important, and perhaps it might tend to move the trajectory away from the fixed point. Numerical experiments showed that the model initialized with the antisymmetric solution would take more than 20 years to move into a lower energy state. Therefore, strong correlation between global wind forcing and an antisym- 
metric solution, which expresses itself in the increased energy input, provides higher chances of reaching and maintaining a high energy state in a time-dependent winddriven model. Such a mechanism for restoring the symmetry of a solution is absent in the boundary-forced model, where the energy input in fixed in time. It is only nonlinear eddy-mean flow and eddy-eddy interactions and dissipation that affect the model trajectory as it moves toward the fixed antisymmetric point. We therefore expect that in the time-dependent colliding jets model, it would be more difficult to reach and, subsequently, to maintain a high energy state.

\subsubsection{Energy Convergence Between the Time Mean Flow and Perturbations}

The energy conversion rates between the time mean flow and perturbations are important for understanding the dynamics of multiple states and of the transition between them. To derive the energy exchange rates, let us divide the potential vorticity and streamfunction fields into the time mean (denoted by bar) and the perturbation (denoted by prime):

$$
\begin{aligned}
q_{i}(x, y, t) & =\bar{q}_{i}(x, y)+q_{i}^{\prime}(x, y, t), \\
\psi_{i}(x, y, t) & =\bar{\psi}_{i}(x, y)+\psi_{i}^{\prime}(x, y, t)
\end{aligned}
$$

where $i=1,2$ are layer indices. Then the potential vorticity equation (3.1) can be rewritten as

$$
\frac{\partial q_{i}^{\prime}}{\partial t}+J\left(\bar{\psi}_{i}, q_{i}^{\prime}\right)+J\left(\psi_{i}^{\prime}, q_{i}^{\prime}\right)+J\left(\bar{\psi}_{i}, \bar{q}_{i}\right)+J\left(\psi_{i}^{\prime}, \bar{q}_{i}\right)=\overline{\mathcal{D}}_{i}+\mathcal{D}_{i}^{\prime}+\overline{\mathcal{S}}_{i}+\mathcal{S}_{i}^{\prime}
$$

Assuming that perturbations are small compared to the time mean fields, the linearized perturbation potential vorticity equation has the following form:

$$
\frac{\partial q_{i}^{\prime}}{\partial t}+J\left(\bar{\psi}_{i}, q_{i}^{\prime}\right)+J\left(\psi_{i}^{\prime}, \bar{q}_{i}\right)=\mathcal{D}_{i}^{\prime}+\mathcal{S}_{i}^{\prime}
$$




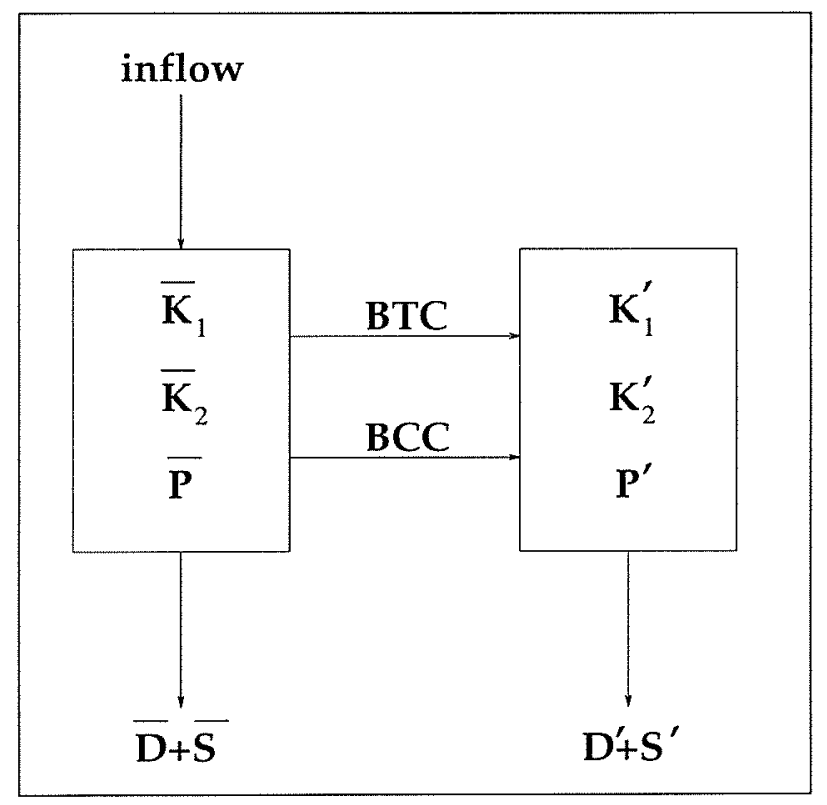

Figure 3-6: General energy conversion diagram for the 2-layer model.

Multiplying equation (3.23) by $-\psi_{i}^{\prime} d_{i}$ and summing over two layers give the following equation for perturbation energy density:

$$
\begin{aligned}
\frac{\partial}{\partial t}\left[\sum_{i=1}^{2} K_{i}^{\prime}+P^{\prime}\right]= & \frac{\partial}{\partial x} \sum_{i=1}^{2} d_{i} \psi_{i}^{\prime}\left[\frac{\partial^{2} \psi_{i}^{\prime}}{\partial t \partial x}+\frac{\beta}{2} \psi_{i}^{\prime}+J\left(\psi_{i}^{\prime}, \frac{\partial \bar{\psi}_{i}}{\partial x}\right)+J\left(\bar{\psi}_{i}, \frac{\partial \psi_{i}^{\prime}}{\partial x}\right)\right]+ \\
& \frac{\partial}{\partial y} \sum_{i=1}^{2} d_{i} \psi_{i}^{\prime}\left[\frac{\partial^{2} \psi_{i}^{\prime}}{\partial t \partial y}+J\left(\psi_{i}^{\prime}, \frac{\partial \bar{\psi}_{i}}{\partial y}\right)+J\left(\bar{\psi}_{i}, \frac{\partial \psi_{i}^{\prime}}{\partial y}\right)\right]- \\
& \sum_{i=1}^{2} \frac{\partial \psi_{i}^{\prime}}{\partial x}\left[J\left(\psi_{i}^{\prime}, \frac{\partial \bar{\psi}_{i}}{\partial x}\right)+J\left(\bar{\psi}_{i}, \frac{\partial \psi_{i}^{\prime}}{\partial x}\right)\right]- \\
& \sum_{i=1}^{2} \frac{\partial \psi_{i}^{\prime}}{\partial y}\left[J\left(\psi_{i}^{\prime}, \frac{\partial \bar{\psi}_{i}}{\partial y}\right)+J\left(\bar{\psi}_{i}, \frac{\partial \psi_{i}^{\prime}}{\partial y}\right)\right]+ \\
& F_{0}\left(\psi_{1}^{\prime}-\psi_{2}^{\prime}\right)\left[J\left(\psi_{1}^{\prime}, \bar{\psi}_{2}\right)-J\left(\psi_{2}^{\prime}, \bar{\psi}_{1}\right)\right]- \\
& \sum_{i=1}^{2} d_{i} \psi_{i}^{\prime}\left(\mathcal{D}_{i}^{\prime}+\mathcal{S}_{i}^{\prime}\right)
\end{aligned}
$$

where $K_{i}^{\prime}=\frac{d_{i}}{2}\left[\left(\frac{\partial \psi_{i}^{\prime}}{\partial x}\right)^{2}+\left(\frac{\partial \psi_{i}^{\prime}}{\partial y}\right)^{2}\right]$ is eddy kinetic energy density and $P^{\prime}=\frac{F_{0}}{2}\left(\psi_{1}^{\prime}-\psi_{2}^{\prime}\right)^{2}$ is eddy potential energy density.

Since the perturbation streamfunction is zero on the boundaries, all the divergence terms of equation (3.24) disappear in the domain integral. Terms responsible 
for time changes in the perturbation energy are the barotropic energy conversion rate, $B T C$, between the mean flow and perturbations due to work by eddy momentum fluxes and the baroclinic conversion rate, $B C C$, between the mean flow and perturbation due to work by eddy heat fluxes:

$$
\begin{aligned}
\operatorname{BTC}(x, y)= & \sum_{i=1}^{2}-d_{i} \frac{\partial \psi_{i}^{\prime}}{\partial x}\left[J\left(\psi_{i}^{\prime}, \frac{\partial \bar{\psi}_{i}}{\partial x}\right)+J\left(\bar{\psi}_{i}, \frac{\partial \psi_{i}^{\prime}}{\partial x}\right)\right]- \\
& d_{i} \frac{\partial \psi_{i}^{\prime}}{\partial y}\left[J\left(\psi_{i}^{\prime}, \frac{\partial \bar{\psi}_{i}}{\partial y}\right)+J\left(\bar{\psi}_{i}, \frac{\partial \psi_{i}^{\prime}}{\partial y}\right)\right] \\
B C C(x, y)= & F_{0}\left(\psi_{1}^{\prime}-\psi_{2}^{\prime}\right)\left[J\left(\psi_{1}^{\prime}, \bar{\psi}_{2}\right)-J\left(\psi_{2}^{\prime}, \bar{\psi}_{1}\right)\right] .
\end{aligned}
$$

When the conversion rates integrated over the model domain are positive, the energy associated with perturbations grows in time, and therefore eddies draw the energy from the mean flow. As we noted earlier, the rate of external forcing due to inflow boundary conditions is given by the energy flux vector $\nabla \cdot \mathbf{P}$, where $\mathbf{P}$ is defined in (3.16)-(3.17). The dissipation rate for the mean flow and for the perturbation are

$$
-\sum_{i=1}^{2} d_{i} \bar{\psi}_{i}\left(\overline{\mathcal{D}}_{i}+\overline{\mathcal{S}}_{i}\right)
$$

and

$$
-\sum_{i=1}^{2} d_{i} \psi_{i}^{\prime}\left(\mathcal{D}_{i}^{\prime}+\mathcal{S}_{i}^{\prime}\right) .
$$

Integrals of the above conversion rates over the model domain are summarized in the energy conversion diagram in Figure 3-6.

\subsection{Ring Dynamics at the Western Wall}

The behavior of rings and their interaction with the western boundary currents may be crucial the stability of the system and therefore on the transition between multiple dynamical states. 


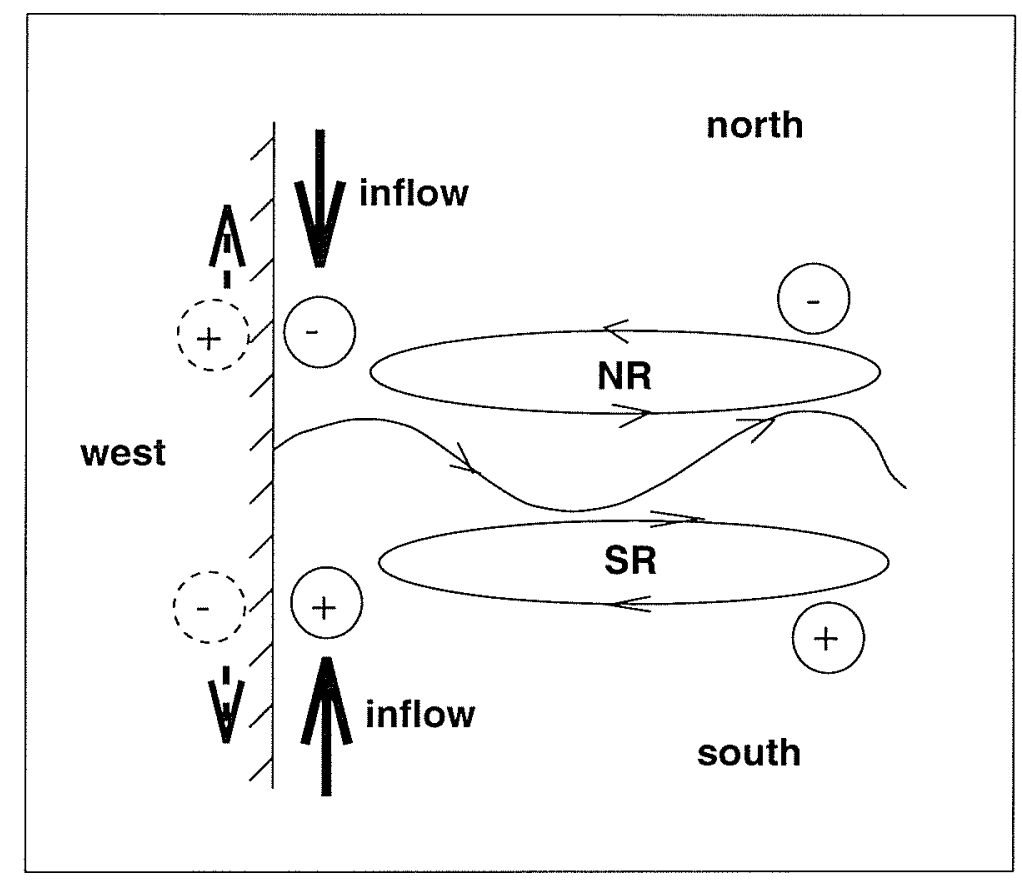

Figure 3-7: A schematic illustrating the behavior of rings on the western boundary.

A ring trajectory at the western boundary is affected by two competing processes (Figure 3-7). The first one is the formation of an image vortex with the opposite vorticity sign due to a rigid wall boundary condition. A newly created dipole structure tends to propel itself along the boundary northward for anticyclonic rings and southward for cyclonic rings. The second process affecting the ring movement is its advection towards the middle of the domain by the western boundary current. Therefore, depending of the strength of the ring, it either moves towards the eastward jet or away from it.

If the ring is weak enough and therefore is advected towards the middle of the domain, it usually rejoins the eastward jet and may, by injecting anomalous potential vorticity, further perturb it. However, if the ring is strong enough to overcome the advective force of the western boundary current, two scenarios are possible. First, the ring-image vortex dipole moves along the wall until entering an absorbing sponge layer. Such a ring might be able either to halt or to change the path of the western 
boundary current. This, in turn, might break the symmetry of the inflow conditions near the separation point and lead to the overshoot of the opposite flowing boundary current. Second, a weaker ring may dissipate enough energy while traveling along the wall, to reverse its trajectory before reaching the sponge layer and subsequently to join the eastward jet. 


\section{Chapter 4}

\section{Results for Reduced-Gravity Colliding Jets Model}

\subsection{Model Formulation}

\subsubsection{Governing Equation}

This chapter reports the results for the reduced-gravity version of the colliding jet model which isolates the importance of barotropic instability and dissipation for the existence and maintenance of low frequency variability and multiple dynamical regimes of a quasi-geostrophic jet with recirculations. The governing potential vorticity equation is easily deduced from equation (3.1) when the lower layer is assumed infinitely deep and motionless:

$$
\frac{\partial q}{\partial t}+\mathcal{J}(\psi, q)=\mathcal{D}+\mathcal{S} \quad \text { in } \Omega
$$

subject to the boundary conditions:

$$
\psi=\Psi, \quad \nabla^{2} \psi=\mathcal{L}, \quad \nabla^{4} \psi=\mathcal{M} \quad \text { in } \bar{\Omega} .
$$


Here, $\psi=\psi(x, y)$ is a quasi-geostrophic streamfunction, $q=\nabla^{2} \psi+\beta y-\gamma^{2} \psi$ is potential vorticity. The parameter space is reduced to

$$
\left\{\gamma^{2}=\frac{L^{2}}{L_{\text {def }}^{2}}, \beta=\frac{\beta^{\text {dim }} L^{2}}{U}, \mathcal{A}_{2}=\frac{\mathcal{A}_{2}^{\text {dim }} L}{U}, \mathcal{A}_{6}=\frac{\mathcal{A}_{6}^{\text {dim }}}{L^{3} U}\right\}
$$

since the depth ratio, $\delta$, and the baroclinic coefficient, $\alpha$, are zero. Coefficient $\mathcal{A}_{2}$ represents the interfacial friction, and therefore the dissipation in the upper layer is given by $\mathcal{D}=-\mathcal{A}_{2} \nabla^{2} \psi-\mathcal{A}_{6} \nabla^{6} \psi$. The model geometry and the structure of the sponge layer are as described in Chapter 3.

\subsubsection{Energetics}

For the case of infinitely deep and motionless lower layer, the energy equation is

$$
\frac{\partial E}{\partial t}+\nabla \cdot \mathbf{P}=G
$$

Here, $E$ is the total energy defined by the sum of the kinetic and available potential energy:

$$
E=\frac{1}{2}\left\{\left(\frac{\partial \psi}{\partial x}\right)^{2}+\left(\frac{\partial \psi}{\partial y}\right)^{2}+\gamma^{2} \psi^{2}\right\} .
$$

The $x$ - and $y$ - components of the energy flux vector, $\mathbf{P}=P_{(x)} \mathbf{i}+P_{(y)} \mathbf{j}$, are defined as

$$
P_{(x)}=-\psi \frac{\partial v}{\partial t}-\psi \Pi u-\frac{\beta}{2} \psi^{2}
$$

and

$$
P_{(y)}=\psi \frac{\partial u}{\partial t}-\psi \Pi v
$$

where $\Pi=q-\beta y$. The dissipation term is given by

$$
G=-\psi(\mathcal{D}+\mathcal{S})
$$




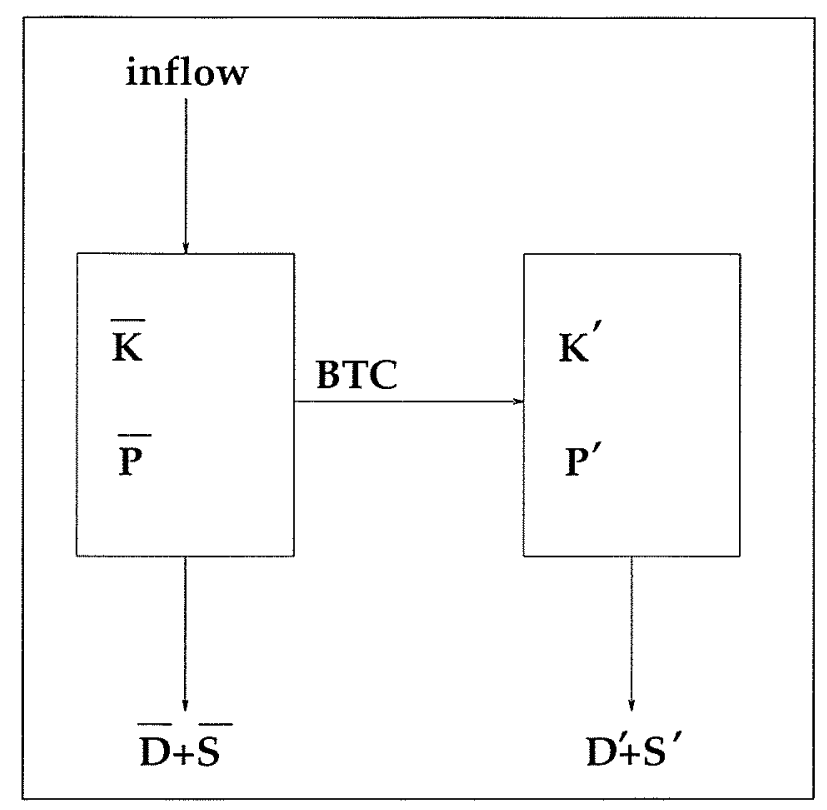

Figure 4-1: Energy diagram for the reduced-gravity model.

Since there is no horizontal heat transfer, the baroclinic conversion term between the perturbation and the time mean state is zero. It is only due to work by Reynolds stresses that there is an energy exchange between the mean flow and perturbations, given by the barotropic conversion rate:

$$
\begin{aligned}
\operatorname{BTC}(x, y)= & \frac{\partial \psi^{\prime}}{\partial x}\left[J\left(\psi^{\prime}, \frac{\partial \bar{\psi}}{\partial x}\right)+J\left(\bar{\psi}, \frac{\partial \psi^{\prime}}{\partial x}\right)\right]+ \\
& \frac{\partial \psi^{\prime}}{\partial y}\left[J\left(\psi^{\prime}, \frac{\partial \bar{\psi}}{\partial y}\right)+J\left(\bar{\psi}, \frac{\partial \psi^{\prime}}{\partial y}\right)\right]
\end{aligned}
$$

The energy input due to inflow boundary conditions is fixed in time and is given by domain-integrated divergence of the energy flux vector, $\mathbf{P}$. The dissipation rate for the mean flow and for perturbations are $-\bar{\psi}(\overline{\mathcal{D}}+\overline{\mathcal{S}})$ and $-\psi^{\prime}\left(\mathcal{D}^{\prime}+\mathcal{S}^{\prime}\right)$ respectively. The energy conversion diagram (Figure 4-1) summarizes the domain-integrated energy transfer rates for the reduced-gravity model. 


\subsection{Linear Stability Analysis of Steady Antisym- metric Solutions}

\subsubsection{Steady States vs Interfacial Friction Coefficient}

In this section, we describe steady antisymmetric solutions of the reduced-gravity model. As we show later, such solutions closely resemble variability associated with a high energy state of the time-dependent model. Therefore, the linear stability characteristics of these solutions are important for understanding the eddy-mean flow interaction present in a high energy state.

Steady antisymmetric solutions of the reduced-gravity model were calculated by adapting a version of the numerical code with enforced meridional antisymmetry. On each iteration, the potential vorticity field was stepped forward in time only for the southern half of the domain, $y<0$, subject to $\psi=0$ at $y=0$. The fields for the northern half of the domain, $y>0$, were taken as mirror images of the fields for $y<0$. The model was integrated until the following numerical equivalent of an equilibration criteria was satisfied:

$$
\frac{|E(t+\Delta t)-E(t)|}{E(t)}<10^{-9} \Delta t,
$$

where $E(t)$ is total energy at time $t$ and $\Delta t$ is the time step.

Figure 4-2 presents steady antisymmetric solutions of the reduced-gravity model for $\mathcal{A}_{6}^{\text {dim }}=8 \cdot 10^{10} \mathrm{~m}^{4} \mathrm{sec}^{-1}, T_{i n}=16 \mathrm{~Sv}$ and the following values of the interfacial fric-

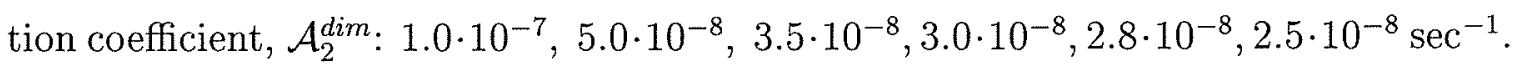
With an increase in the dimensional interfacial friction coefficient, $\mathcal{A}_{2}^{\text {dim }}$, the total energy level of the steady solutions diminishes from 0.08 to 0.052 (Figure 4-3a), which is directly related to a decrease from 2350 to $1000 \mathrm{~km}$ in the longitudinal size of the recirculating gyres (Figure 4-3b). The maximum in the total eastward (westward) transport also drops from 41 to 26 (31 to 16) Sv (Figure 4-3c,d), indicating weaker eastward jet and recirculations. 
(a)

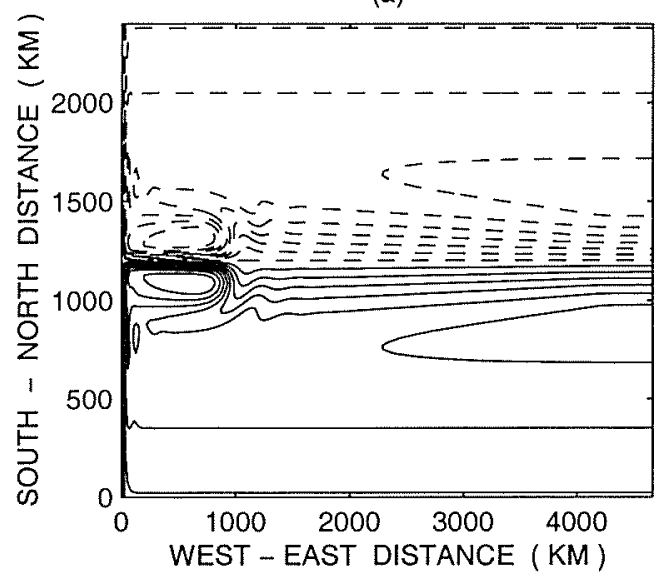

(c)

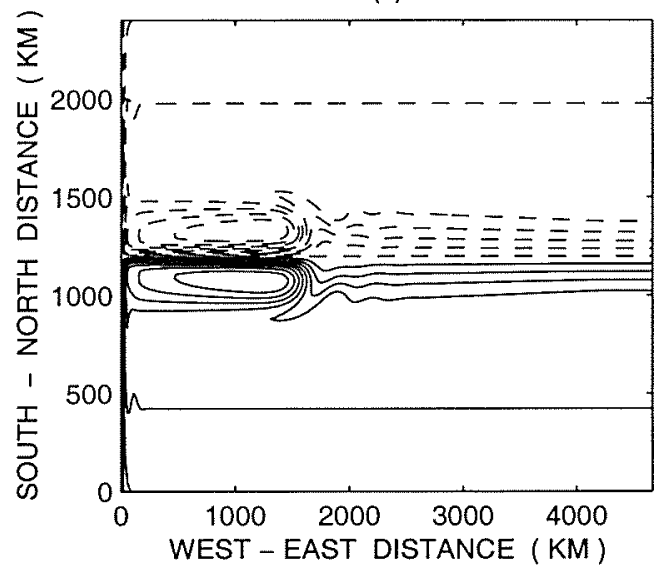

(e)

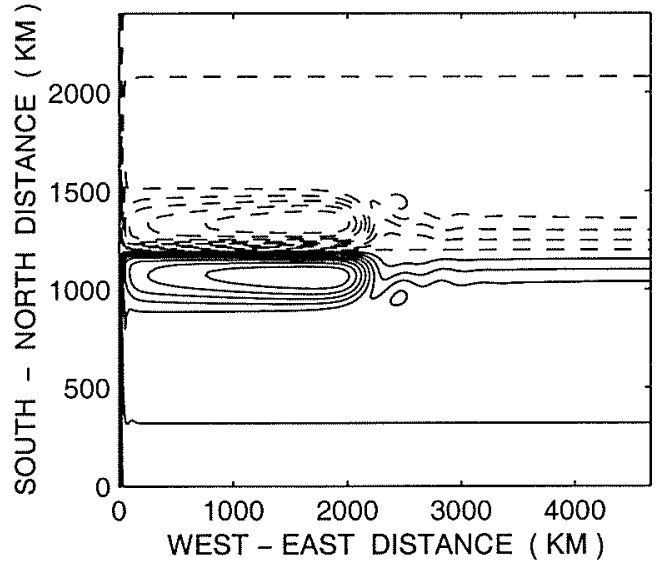

(b)

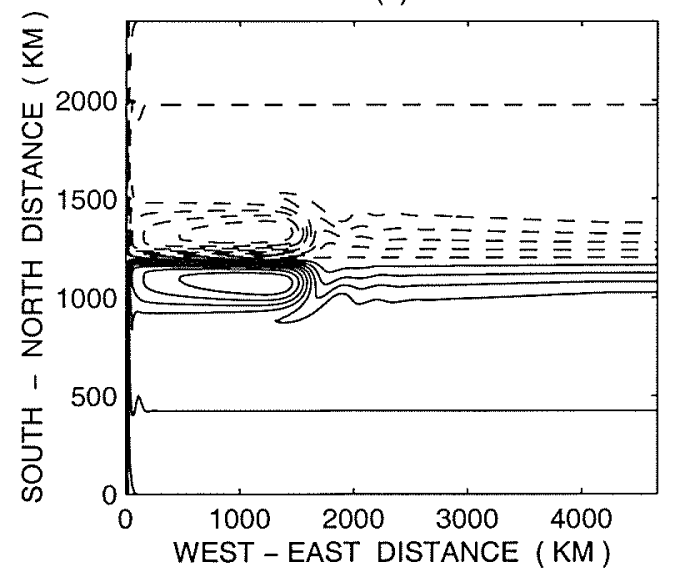

(d)

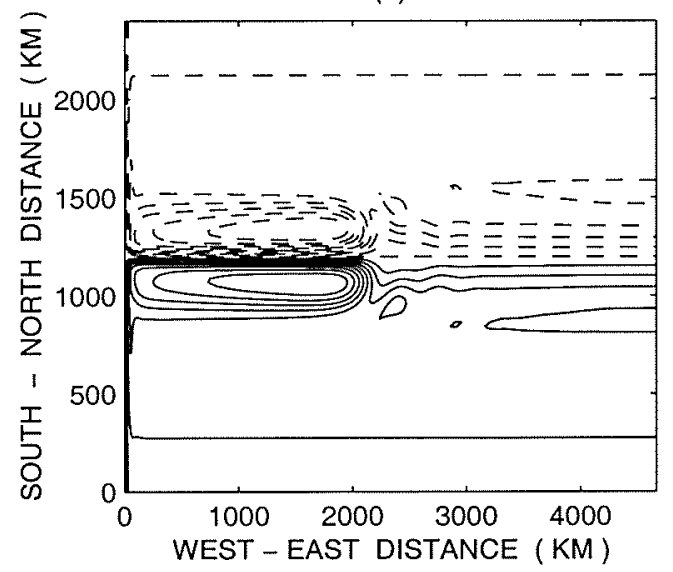

(f)

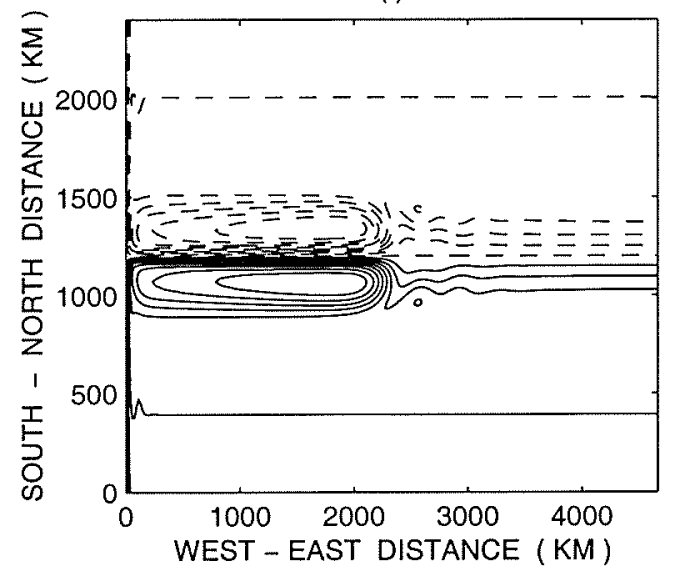

Figure 4-2: Streamfunction contours of steady antisymmetric solutions for $\mathcal{A}_{2}^{\text {dim }}=$ $1.0 \cdot 10^{-7} \mathrm{sec}^{-1}$ (a), $5.0 \cdot 10^{-8} \mathrm{sec}^{-1}$ (b), $3.5 \cdot 10^{-8} \mathrm{sec}^{-1}$ (c), $3.0 \cdot 10^{-8} \mathrm{sec}^{-1}$ (d), $2.8 \cdot 10^{-8} \mathrm{sec}^{-1}(\mathrm{e}), 2.5 \cdot 10^{-8} \mathrm{sec}^{-1}(\mathrm{f})$. Parameters: $T_{\text {in }}=16 \mathrm{~Sv}, \mathcal{A}_{6}^{\operatorname{dim}}=8 \cdot 10^{10} \mathrm{~m}^{4}$ $\sec ^{-1}$. 
(a)

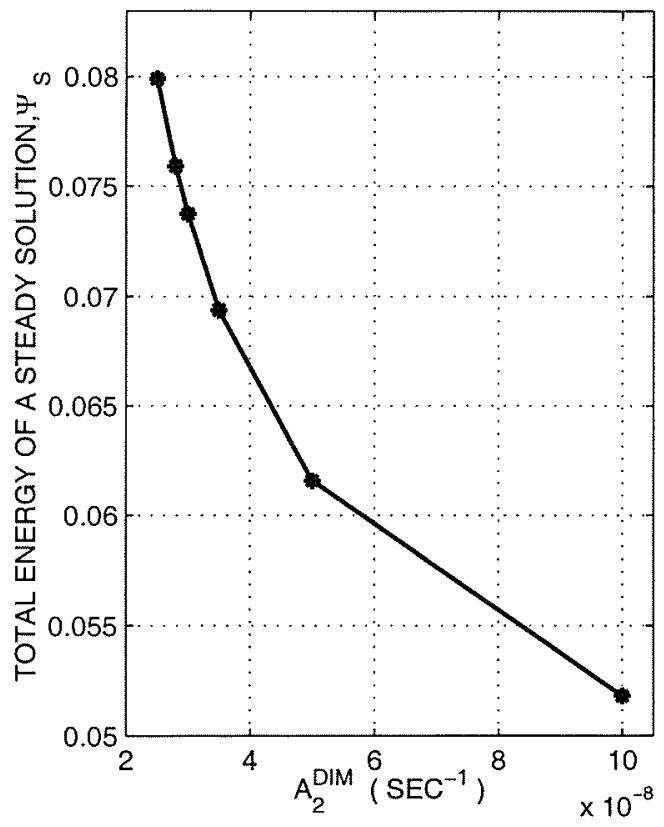

(c)

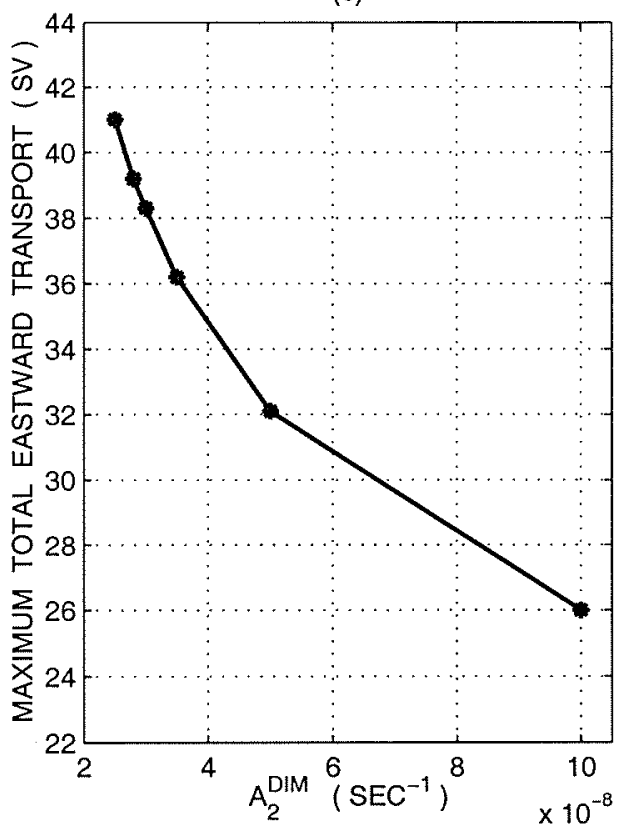

(b)

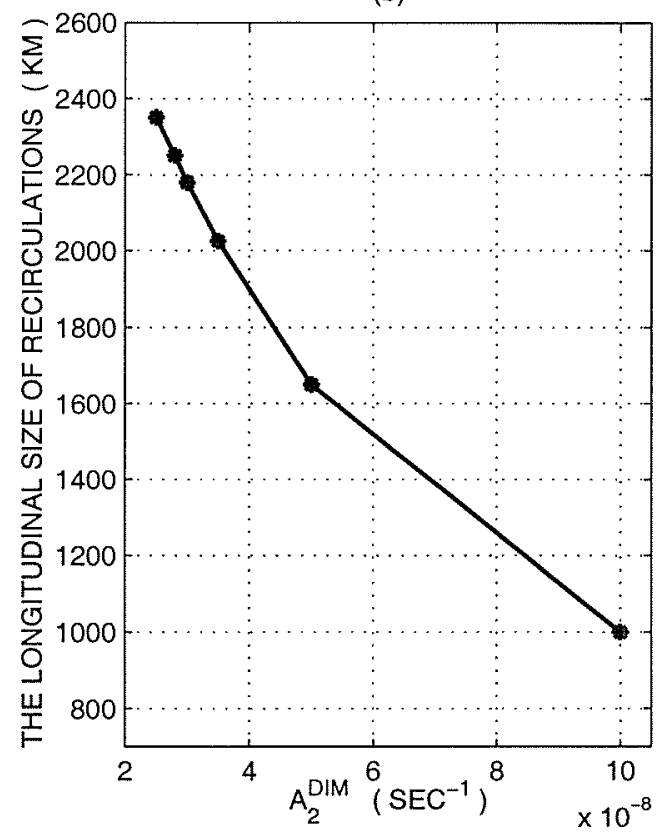

(d)

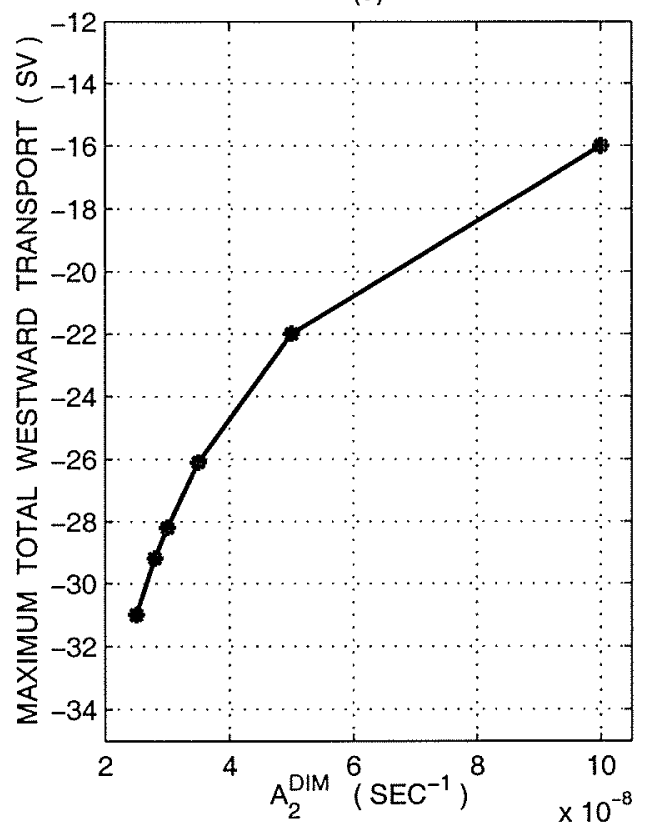

Figure 4-3: Total nondimensional energy (a), the longitudinal size of the recirculations (b), and maxima in the total eastward (c) and westward (d) transport of steady antisymmetric solutions, $\Psi_{S}$, of the reduced-gravity model as functions of dimensional interfacial drag coefficient, $\mathcal{A}_{2}^{\text {dim }}$. Parameters: $T_{i n}=16 \mathrm{~Sv}, \mathcal{A}_{6}^{\text {dim }}=8 \cdot 10^{10} \mathrm{~m}^{4} \mathrm{sec}^{-1}$. 


\subsubsection{Growth Rates, Phase Speeds and Structures of Unstable Modes}

To determine the growth rates, phase speeds and spatial structures of growing modes associated with steady state solutions, we used an initial value technique applied to the original nonlinear model. A similar approach was adopted by Simmons and Hoskins [36] in a study of quasi-geostrophic baroclinic instabilities on a sphere. The model was initialized with the sum of a steady state solution, $\Psi_{S}$, and a uniformly distributed random noise, $\psi^{\prime}(x, y)$. The amplitude of the perturbation potential vorticity field was chosen as $1 \%$ of the maximum in the steady state potential vorticity field, $Q_{S}$.

Let us represent the perturbation streamfunction as a modal solution of the form $\psi^{\prime}(x, y, t)=$ Real $A(x, y) e^{\left(\sigma_{r}+i \sigma_{i}\right) t}$, where $A(x, y)=A_{r}(x, y)+i A_{i}(x, y)$ is a spatially varying amplitude, $A_{r}$ and $A_{i}$ are real functions, $\sigma_{r}$ is a growth rate and $\sigma_{i}$ is frequency. The total energy of the perturbation field is given by

$$
E\left(\psi^{\prime}(x, y, t)\right)=e^{2 \sigma_{r} t} \frac{1}{2}\left\{\left|\frac{\partial B}{\partial x}\right|^{2}+\left|\frac{\partial B}{\partial y}\right|^{2}+\gamma^{2}|B|^{2}\right\},
$$

where $B=A_{r} \cos \sigma_{i} t-A_{i} \sin \sigma_{i} t+i\left(A_{i} \cos \sigma_{i} t+A_{r} \sin \sigma_{i}\right)$. Assuming that the perturbation amplitude is small, and therefore the nature of the eddy-mean flow interaction is linear, the growth rate can be estimated by fitting a straight line to the natural logarithm of the perturbation energy:

$$
\ln E\left(\psi^{\prime}(x, y, t)\right)=2 \sigma_{r} t+\ln \left\{\left|\frac{\partial B}{\partial x}\right|^{2}+\left|\frac{\partial B}{\partial y}\right|^{2}+\gamma^{2}|B|^{2}\right\} .
$$

When $\mathcal{A}_{2}^{\text {dim }}=1.0 \cdot 10^{-7} \mathrm{sec}^{-1}$, the perturbation energy decays to zero with time, and therefore the corresponding steady antisymmetric solution is stable. However, for $\mathcal{A}_{2}^{\text {dim }} \geq 5.0 \cdot 10^{-8} \mathrm{sec}^{-1}$, steady antisymmetric solutions are unstable, and the time evolution of $\ln E\left(\psi^{\prime}(x, y)\right)$ (Figure 4-4a) is characterized by three distinct regions which reflect changes in the nature of internal interactions. Initially, the uniformly distributed perturbation field does not correspond to a preferred mode of instability 
(a)

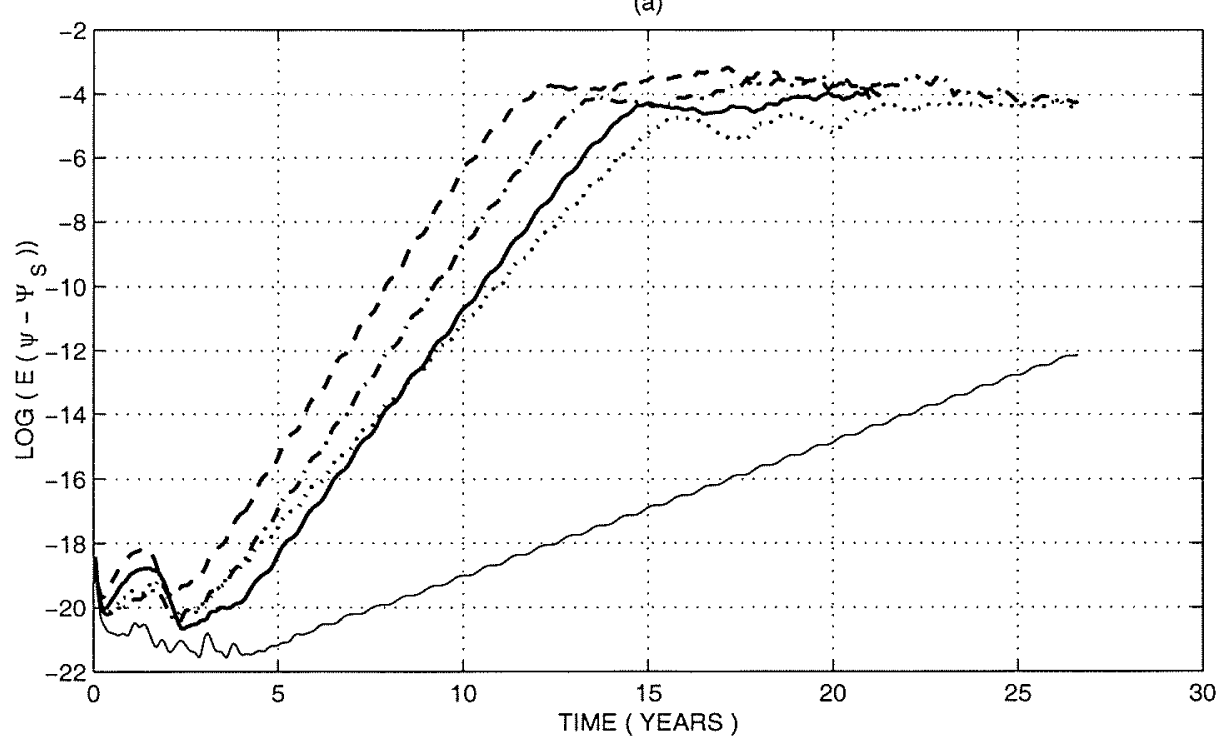

(b)

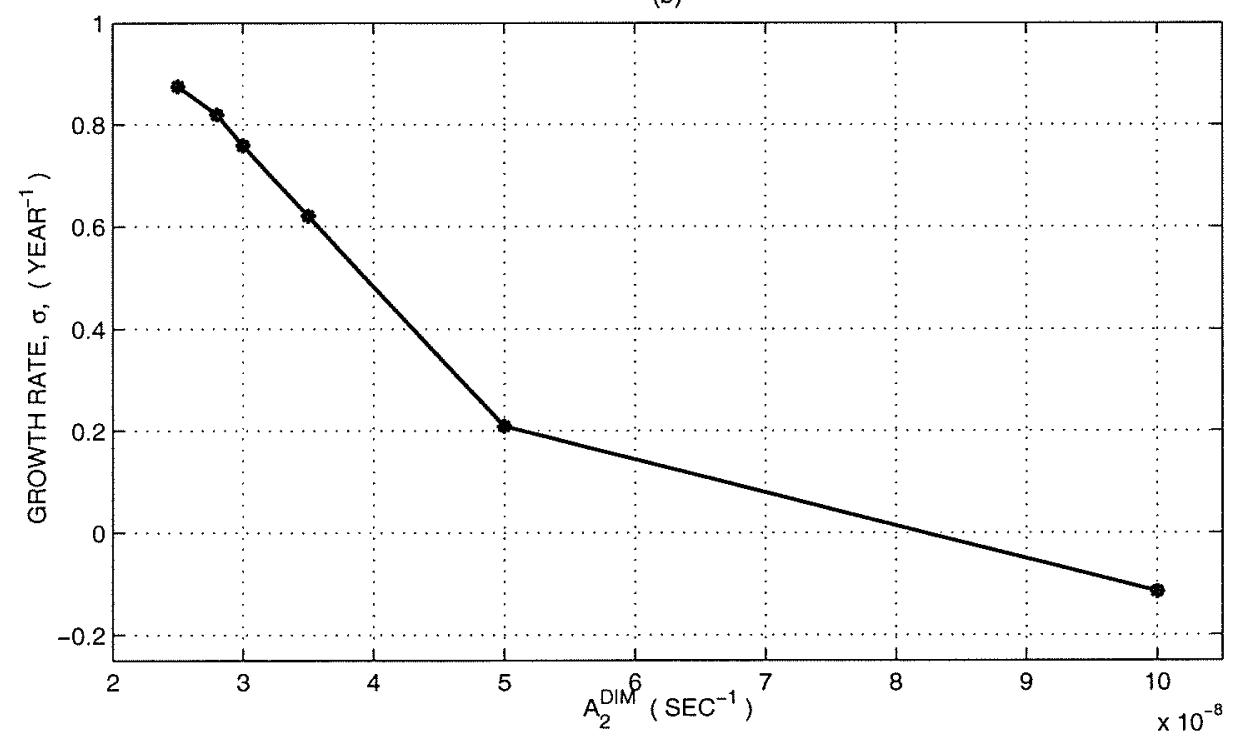

Figure 4-4: (a): Natural logarithm of energy of $\psi^{\prime}=\psi-\Psi_{S}$ as a function of time, where $\Psi_{S}$ is an antisymmetric steady solution and $\psi$ is the solution of the timedependent model initialized with a perturbed steady solution. Interfacial friction coefficient: $\mathcal{A}_{2}=5.0 \cdot 10^{-8} \mathrm{sec}^{-1}$ (thin solid line), $3.5 \cdot 10^{-8} \mathrm{sec}^{-1}$ (dotted line), $3.0 \cdot 10^{-8} \mathrm{sec}^{-1}$ (thick solid line), $2.8 \cdot 10^{-8} \mathrm{sec}^{-1}$ (dashed-dotted line), $2.5 \cdot 10^{-8} \mathrm{sec}^{-1}$ (dashed line). Parameters: $T_{i n}=16 \mathrm{~Sv}, \mathcal{A}_{6}^{\text {dim }}=8 \cdot 10^{10} \mathrm{~m}^{4} \mathrm{sec}^{-1}$. (b): Growth rate, $\sigma_{r}$, (in year ${ }^{-1}$ ) as a function of the dimensional interfacial drag coefficient, $\mathcal{A}_{2}^{\text {dim }}$. 
and therefore is not efficient at extracting energy from the mean flow. After 3 or 4 years, the exponentially growing mode arises on the axis of the eastward jet and in the area of recirculations, and hence $\ln E\left(\psi^{\prime}(x, y, t)\right)$ displays linear growth. For $\mathcal{A}_{2}^{\text {dim }} \leq 3.5 \cdot 10^{-8} \mathrm{sec}^{-1}$, the perturbation amplitude becomes so large after 13 to 15 years, that the nonlinear eddy-eddy interactions become dominant, and the growth rate saturates. Notice that for $\mathcal{A}_{2}^{\mathrm{dim}}=5.0 \cdot 10^{-8} \mathrm{sec}^{-1}$, the growth rate was still linear after 27 years. This indicates that a trajectory of the time-dependent model might stay in the vicinity of the fixed point associated with the antisymmetric solution for a long time. In general, there might be several unstable, exponentially growing modes in the system, which would then enforce curvature of $\ln E\left(\psi^{\prime}(x, y, t)\right)$ during a stage of exponential growth. The absence of such curvature in $\ln E\left(\psi^{\prime}(x, y, t)\right)$ (Figure 4-4a) in the present model indicates the existence of just one unstable mode.

The e-folding time, $1 / \sigma$, of the unstable, oscillating mode decreases from 4.8 years for $\mathcal{A}_{2}^{\text {dim }}=5.0 \cdot 10^{-8} \mathrm{sec}^{-1}$ to 417 days for $\mathcal{A}_{2}^{\text {dim }}=2.5 \cdot 10^{-8} \mathrm{sec}^{-1}$ (Figure $4-4 \mathrm{~b})$. As we show later, the time-dependent regional model displays multiple dynamical regimes when $\mathcal{A}_{2}^{\text {dim }}=3 \cdot 10^{-8}$. The corresponding e-folding time for the nearly antisymmetric solution is 481 days. In comparison, Primeau [29] estimated the e-folding time at 897 days for the antisymmetric steady state solution of the winddriven reduced-gravity model with the parameter set from McCalpin and Haidvogel's model [25]. The differences in the e-folding times between the present model and the wind-driven case could be attributed to the differences in the strength of forcing.

Snapshots of the most unstable modes (Figure 4-5) show a spatial structure similar to that calculated by Primeau [29]. There are four cells on the axis of the eastward jet and weaker cells in the area of westward recirculations. Visualization of the mode's time series reveals a very complicated propagation pattern, when amplitudes and spatial extents of the cells located on the jet axis slowly grow at some times and quickly shift westward at other times. The westward propagation speed of the cells in the area of the recirculations is constant in time. Qualitative similarity 
(a)
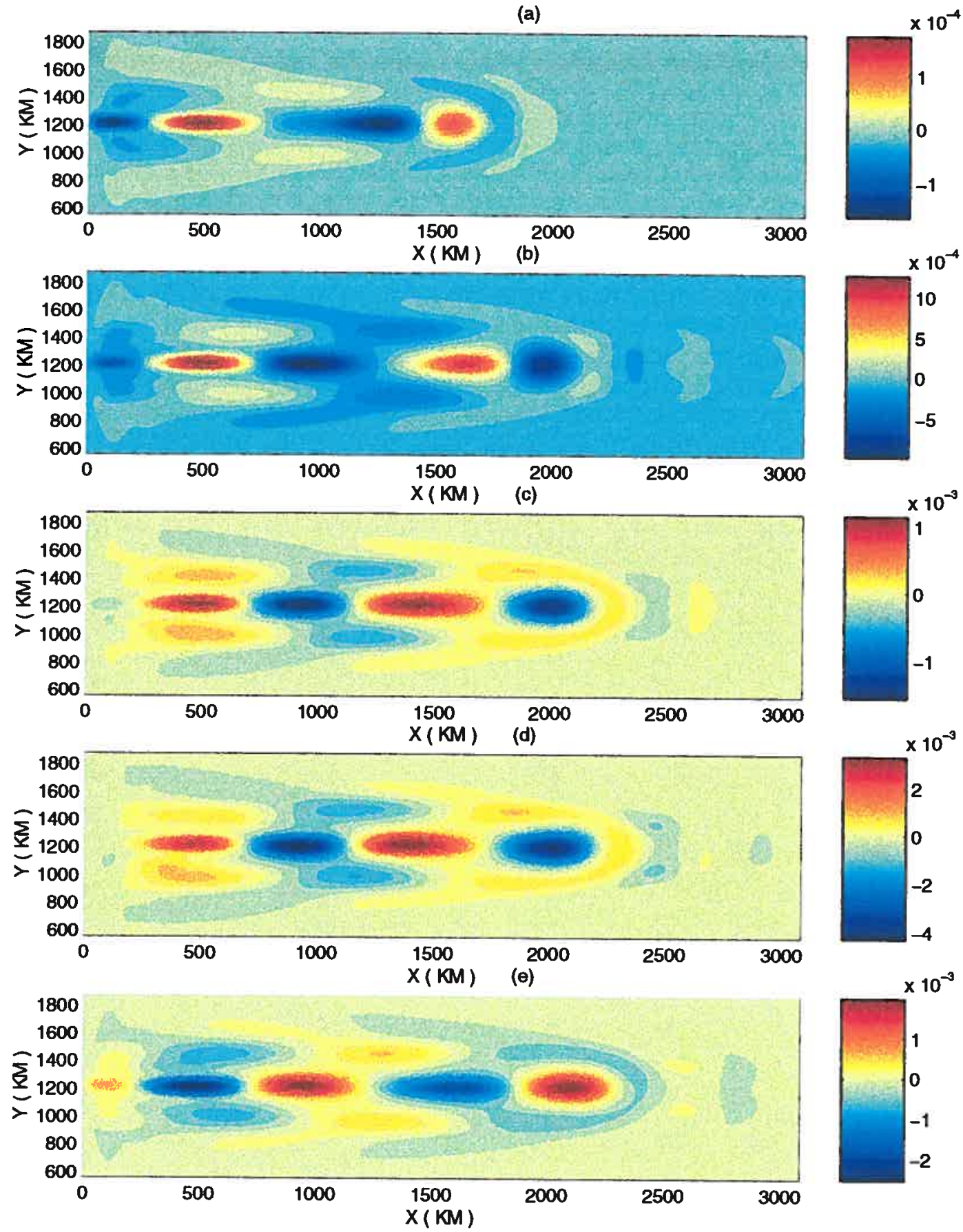

Figure 4-5: Snapshots of exponentially growing modes for $\mathcal{A}_{2}^{\text {dim }}$ equal $5.0 \cdot 10^{-8} \mathrm{sec}^{-1}$ (a), 3.5 $10^{-8} \sec ^{-1}$ (b), $3.0 \cdot 10^{-8} \sec ^{-1}$ (c), $2.8 \cdot 10^{-8} \sec ^{-1}$ (d), $2.5 \cdot 10^{-8} \sec ^{-1}$ (e). Parameters: $T_{\text {in }}=16 \mathrm{~Sv}, \mathcal{A}_{6}^{\text {dim }}=8 \cdot 10^{10} \mathrm{~m}^{4} \mathrm{sec}^{-1}$. Snapshots are presented only for a sub-domain of the model. 
between the results of linear stability analysis of the antisymmetric solutions for the regional and the wind-driven model stems from the fact that wind-forcing drops out of the linearized equations of motion for the wind-driven case.

When nonlinearities become large, the potential vorticity anomalies associated with four cells start interacting with each other leading to saturation in the energy growth and to formation of rings in the area east of the recirculations. This process might be identified as a spatial growth of instabilities.

\subsection{Sensitivity Study for Various Interfacial Fric- tion Coefficients}

\subsubsection{Classification of States}

The regional colliding jet model showed strong dependence of the flow character on the value of the interfacial friction coefficient, $\mathcal{A}_{2}^{d i m}$. Figures $4-6$ and $4-7$ summarize the results of the sensitivity study for the following two values of the biharmonic diffusion coefficient: $\mathcal{A}_{6}^{\text {dim }}=8 \cdot 10^{10} \mathrm{~m}^{4} \mathrm{sec}^{-1}$ and $\mathcal{A}_{6}^{\text {dim }}=8 \cdot 10^{8} \mathrm{~m}^{4} \mathrm{sec}^{-1}$. For both values of $\mathcal{A}_{6}^{\text {dim }}$, with a decrease in $\mathcal{A}_{2}^{\text {dim }}$, the model progressed through the following regimes:

- State $A$. The flow structure consists of a very broad, stable eastward jet; the recirculations are absent.

- State $B$. The flow structure is stable and consists of an eastward jet surrounded by two recirculating gyres.

- State $C$. The flow structure is unstable and consists of an eastward jet surrounded by two recirculating gyres. The eddy field is dominated by eastward 


\begin{tabular}{|c|c|c|}
\hline $\mathcal{A}_{2}^{\text {dim }}$ & STATE & FLOW CHARACTER \\
\hline $5.0 \cdot 10^{-9}$ & $F$ & $\begin{array}{l}\text { one dynamical state: eastward jet with large-amplitude, } \\
\text { westward propagating meanders and large meridional } \\
\text { shifts in the jet separation point; strong rings are formed } \\
\text { on the western boundary; recirculations are absent } \\
\text { as above }\end{array}$ \\
\hline $4.0 \cdot 10^{-8}$ & $E$ & $\begin{array}{l}\text { low frequency variability associated with two dynami- } \\
\text { cal states: high energy state is characterized by large } \\
\text { / medium / small recirculations and weak / medium / } \\
\text { strong eddy and ring fields, rings are formed east of the } \\
\text { recirculations; low energy state has no recirculations and } \\
\text { is characterized by large-amplitude, westward propagat- } \\
\text { ing meanders, large meridional shifts in the jet separation } \\
\text { point and strong rings formed on the western boundary }\end{array}$ \\
\hline $5.0 \cdot 10^{-8}$ & $E$ & as above \\
\hline $6.0 \cdot 10^{-8}$ & $E$ & as above \\
\hline $7.5 \cdot 10^{-8}$ & $D$ & $\begin{array}{l}\text { low frequency variability associated with eastward jet } \\
\text { and longitudinally pulsating recirculations, strong ring } \\
\text { formation, eastward and westward propagating mean- } \\
\text { ders, weak and medium rings are formed east of the } \\
\text { recirculations }\end{array}$ \\
\hline $1.0 \cdot 10^{-7}$ & $C$ & $\begin{array}{l}\text { one dynamical state: eastward jet and two recirculating } \\
\text { gyres, weak ring formation, eastward and westward prop- } \\
\text { agating meanders }\end{array}$ \\
\hline $1.5 \cdot 10^{-7}$ & $B$ & stable state: eastward jet with two recirculating gyres \\
\hline $2.0 \cdot 10^{-7}$ & $B$ & as above \\
\hline $2.5 \cdot 10^{-7}$ & $A$ & stable state: broad stable jet, no recirculations \\
\hline
\end{tabular}

Figure 4-6: Flow character vs interfacial friction coefficient, $\mathcal{A}_{2}^{\text {dim }}$ (in $\mathrm{sec}^{-1}$ ), when $T_{\text {in }}=16 \mathrm{~Sv}$ and $\mathcal{A}_{6}^{\text {dim }}=8 \cdot 10^{8} \mathrm{~m}^{4} \mathrm{sec}^{-1}$. 


\begin{tabular}{|c|c|c|}
\hline $\mathcal{A}_{2}^{\text {dim }}$ & STATE & FLOW CHARACTER \\
\hline 0 & & $\begin{array}{l}\text { the flow did not reach a steady state after } 60 \text { years of } \\
\text { integration }\end{array}$ \\
\hline $1 \cdot 10^{-8}$ & $F$ & $\begin{array}{l}\text { one dynamical state: eastward jet with large-amplitude, } \\
\text { westward propagating meanders and large meridional } \\
\text { shifts in the jet separation point; strong rings are formed } \\
\text { on the western boundary; recirculations are absent } \\
\text { as above }\end{array}$ \\
\hline $2.5 \cdot 10^{-8}$ & $F$ & as above \\
\hline $2.8 \cdot 10^{-8}$ & $E$ & $\begin{array}{l}\text { low frequency variability associated with two dynami- } \\
\text { cal states: high energy state is characterized by large } \\
\text { / medium / small recirculations and weak / medium / } \\
\text { strong eddy and ring fields, rings are formed east of the } \\
\text { recirculations; low energy state has no recirculations and } \\
\text { is characterized by large-amplitude, westward propagat- } \\
\text { ing meanders, large meridional shifts in the jet separation } \\
\text { point and strong rings formed on the western boundary } \\
\text { as above }\end{array}$ \\
\hline $3.5 \cdot 10^{-8}$ & $D$ & $\begin{array}{l}\text { low frequency variability associated with eastward jet } \\
\text { and longitudinally pulsating recirculations, strong ring } \\
\text { formation, eastward and westward propagating mean- } \\
\text { ders, weak and medium rings are formed east of the } \\
\text { recirculations }\end{array}$ \\
\hline $5.0 \cdot 10^{-8}$ & $C$ & $\begin{array}{l}\text { one dynamical state: eastward jet and two recirculating } \\
\text { gyres, weak ring formation, eastward and westward prop- } \\
\text { agating meanders }\end{array}$ \\
\hline $1.0 \cdot 10^{-7}$ & $B$ & stable state: eastward jet with two recirculating gyres \\
\hline
\end{tabular}

Figure 4-7: Flow character vs interfacial friction coefficient, $\mathcal{A}_{2}^{\text {dim }}$ (in $\sec ^{-1}$ ), when $T_{\text {in }}=16 \mathrm{~Sv}$ and $\mathcal{A}_{6}^{\text {dim }}=8 \cdot 10^{10} \mathrm{~m}^{4} \mathrm{sec}^{-1}$. 
and westward propagating meanders. Weak rings form east of the recirculating gyres.

- State $D$. The flow structure is unstable and consists of an eastward jet and two recirculating gyres. The longitudinal size of the recirculations changes with time thus giving rise to low frequency variability. The amplitude and the spatial structure of the eddy field are strongly time-dependent. The eddy field is characterized by eastward and westward propagating meanders. Their interactions give birth to rings in the area east of the recirculation gyres.

- State E. The flow structure displays well-developed low frequency variability. The model stays in one of the two dynamical regimes: high energy state $D$ or low energy state $F$.

- State $F$. The flow structure is unstable and consists of an eastward jet with large meridional excursions in the separation point. The eddy field is dominated by large-amplitude, westward propagating meanders and strong rings formed only on the western boundary. The recirculations are absent in instantaneous fields.

The existence of multiple energy regimes is illustrated in Figure 4-8, which shows normalized histograms of total energy for various values of the interfacial friction coefficient. The energy level of steady antisymmetric solutions discussed in section 4.2 .1 is denoted by a dashed line and is either equal or higher than that of the corresponding time-dependent solutions. For weakly unstable flows $\left(\mathcal{A}_{2}^{\text {dim }}=\right.$ $\left.5 \cdot 10^{-7} \mathrm{sec}^{-1}\right)$, the trajectory of the time-dependent model stays in the vicinity on a fixed antisymmetric point, and therefore the range of diagnosed energy values is small and is close to $E\left(\Psi_{S}\right)$. The corresponding energy histogram has only one peak, indicating a unique dynamical regime. For stronger unstable flows, the range of energy values of the time-dependent model becomes larger. With further decrease in $\mathcal{A}_{2}^{\text {dim }}$, the energy histogram changes its shape from a normal to a bimodal distribution. The model now has two preferred energy states, each one associated with a 


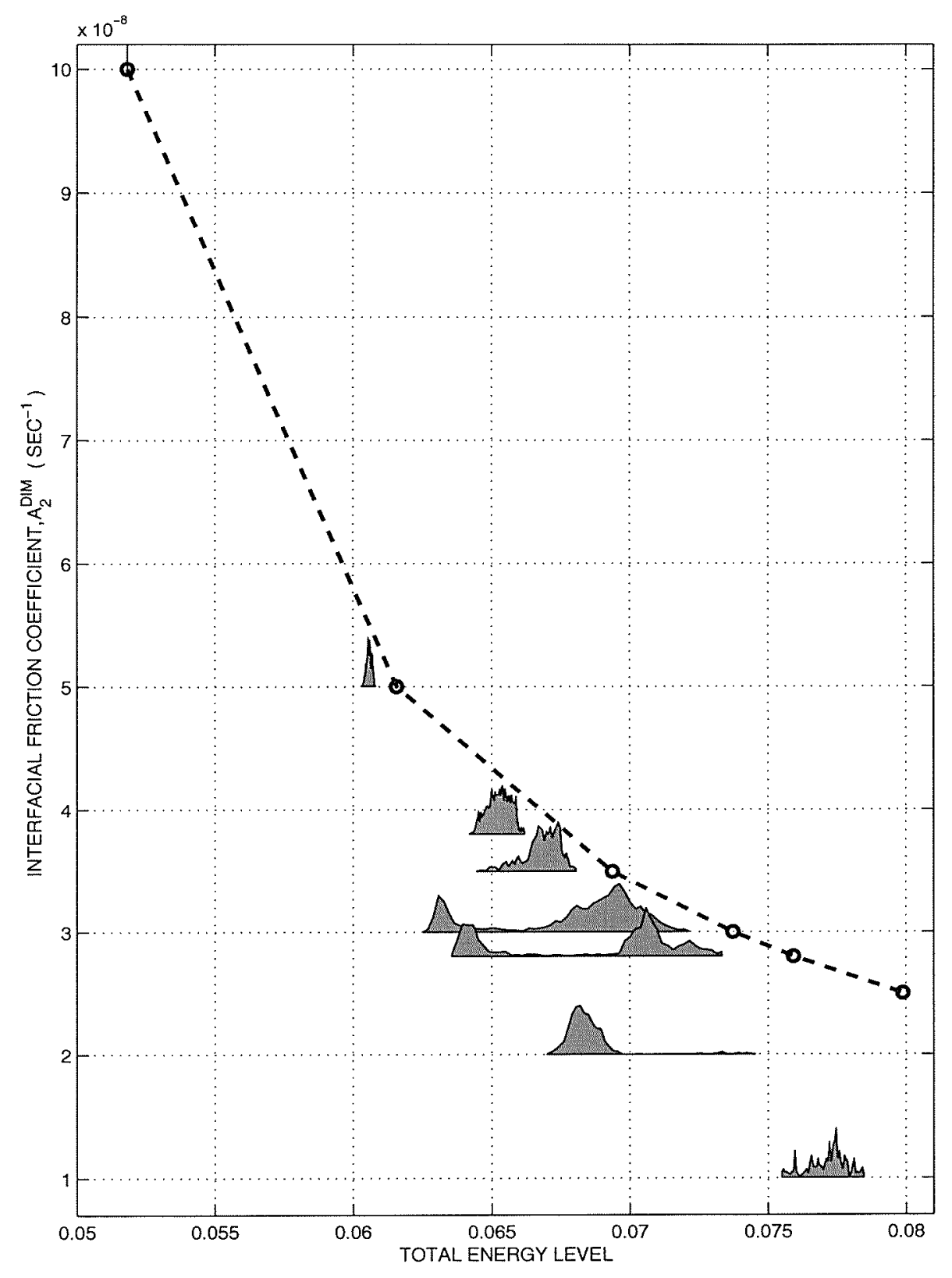

Figure 4-8: Total nondimensional energy level, as diagnosed from the time-dependent model, vs interfacial friction coefficient, $\mathcal{A}_{2}^{\text {dim }}$. Normalized energy histograms are shown on top of each energy distribution. The dashed line denotes the energy level of the antisymmetric steady solutions. 
distinct dynamical regime. For small values of the interfacial friction coefficient, the high energy state becomes unstable, and the model trajectory stays in a dynamical regime associated with a low energy state.

\subsubsection{Examples of States}

In this section, we briefly describe the dynamics of different states. The full analysis of the model will be given in section 4.4 for the case of state $E$.

\section{State $A$}

For high values of the interfacial drag coefficient, the flow has the following structure. Part of the meridional inflow separates from the western boundary current and flows eastward creating a broad stable current that leaves the domain though the eastern boundary. Another part of the flow represents two colliding currents that form a narrow eastward jet that broadens $400-700 \mathrm{~km}$ east of the separation point (Figure 4-9). There are no recirculations in this state, although there are weak $\left(0.2 \mathrm{~m} \mathrm{sec}^{-1}\right)$ westward velocities north and south of the narrow part of the jet. Instead of closing on themselves, contours of streamfunction and potential vorticity loop towards the

western boundary current and then curve back eastward. Even though $\frac{\partial q}{\partial \psi}$ is negative in the eastward jet, therefore satisfying the necessary condition for instability, the interfacial drag is so strong that it suppresses any growing disturbances.

\section{State $B$}

An example of the flow structure for state $B$ is presented in section 4.2.1 (Figure 4-2a) for $\mathcal{A}_{2}^{\text {dim }}=1 \cdot 10^{-7} \mathrm{sec}^{-1}$ and $\mathcal{A}_{6}^{\text {dim }}=8 \cdot 10^{10} \mathrm{~m}^{4} \mathrm{sec}^{-1}$. 
(a)

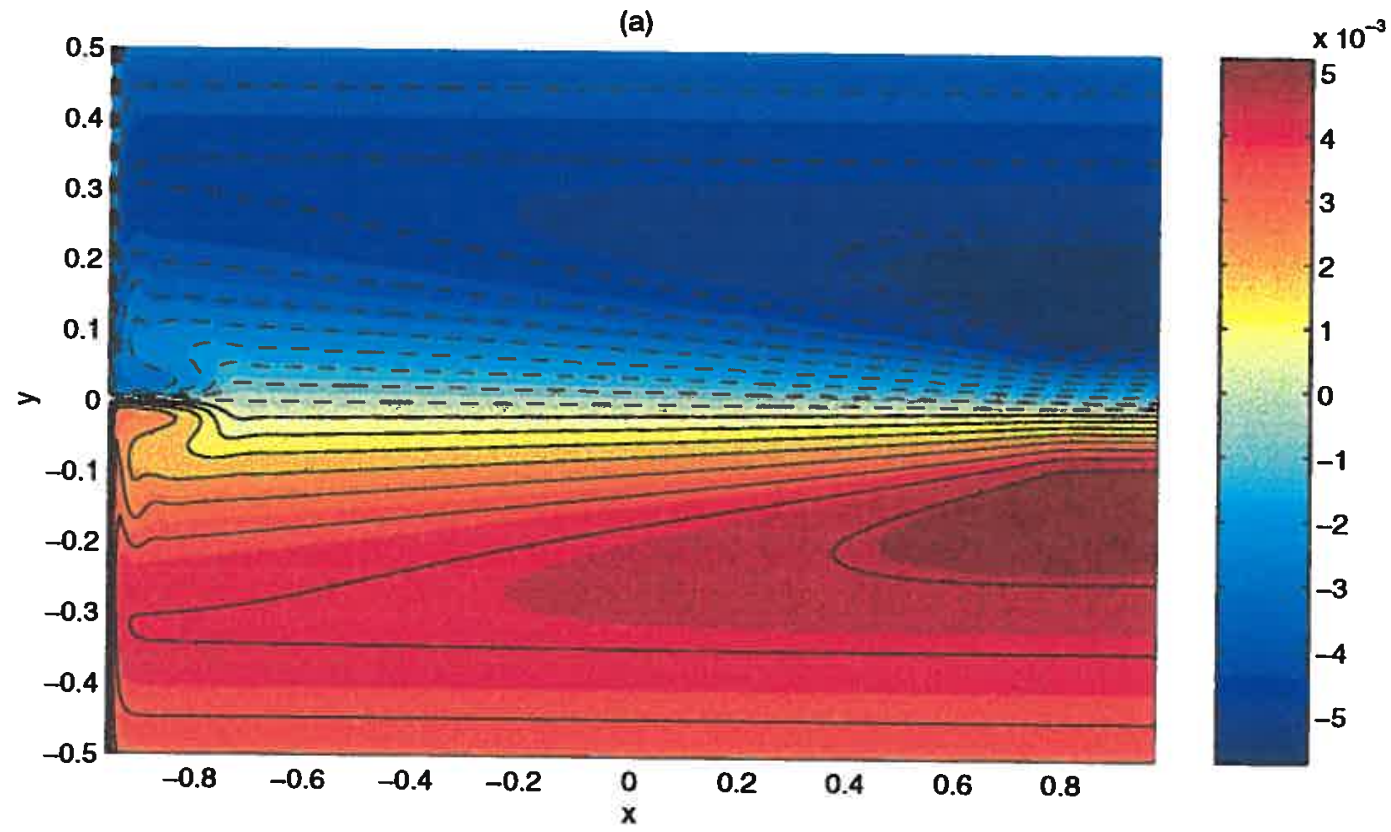

(b)

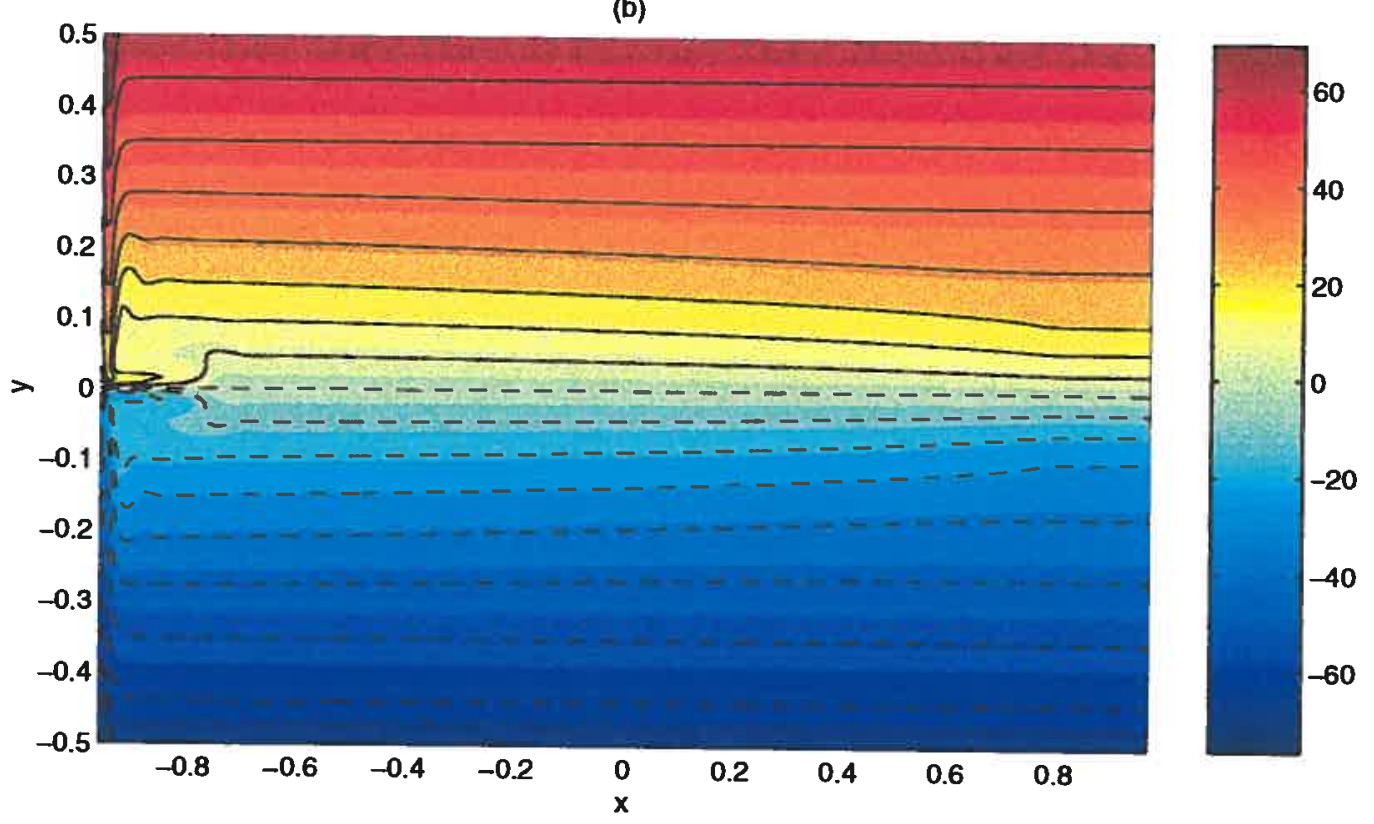

Figure 4-9: Contours of time mean streamfunction (a) and potential vorticity (b) for $\mathcal{A}_{2}^{\text {dim }}=2.5 \cdot 10^{-7} \mathrm{sec}^{-1}$, initial inflow transport of $16 \mathrm{~Sv}$ and $\mathcal{A}_{6}^{\text {dim }}=8 \cdot 10^{8} \mathrm{~m}^{4} \mathrm{sec}^{-1}$. Solid line is used for positive values, dashed line is used for negative values. 
(a)

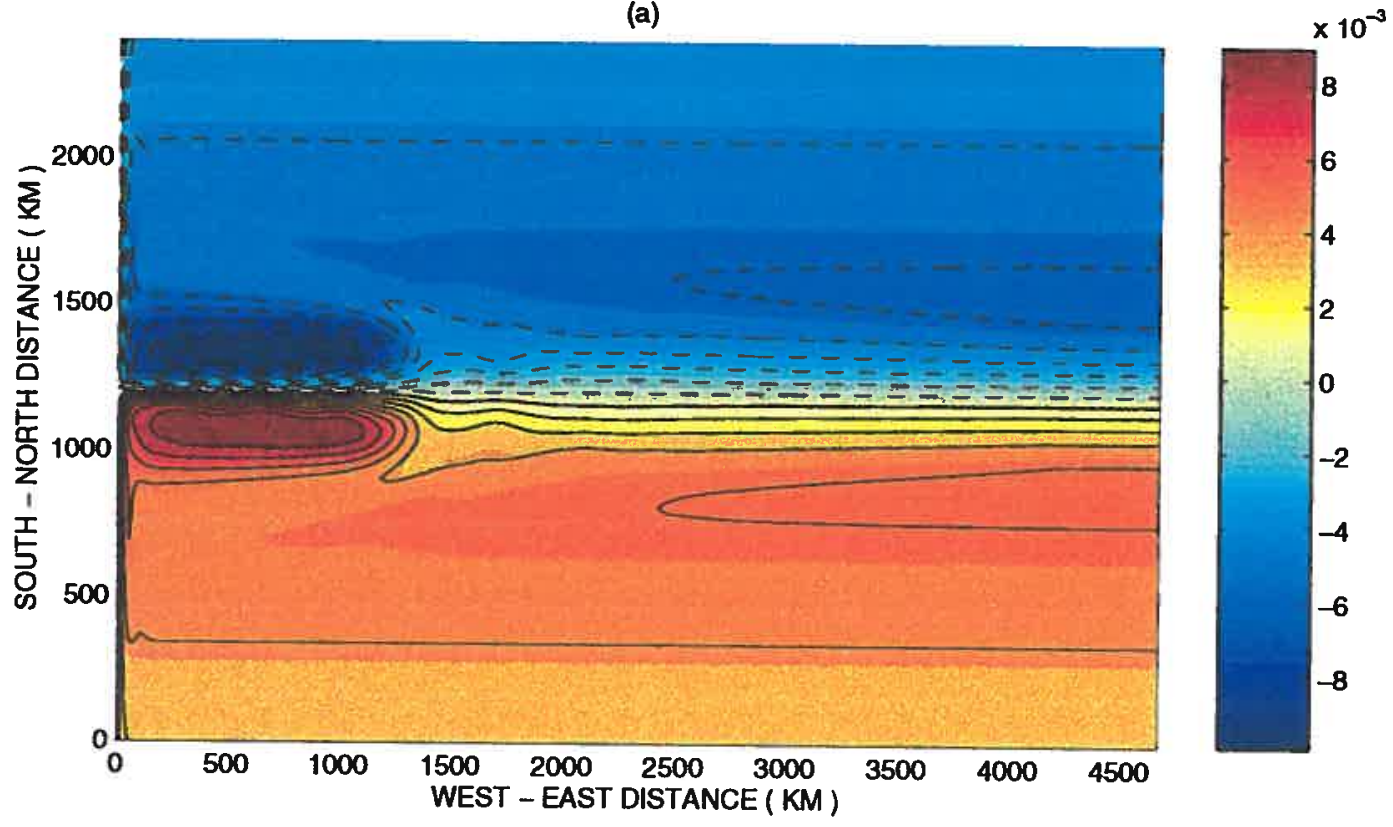

(b)

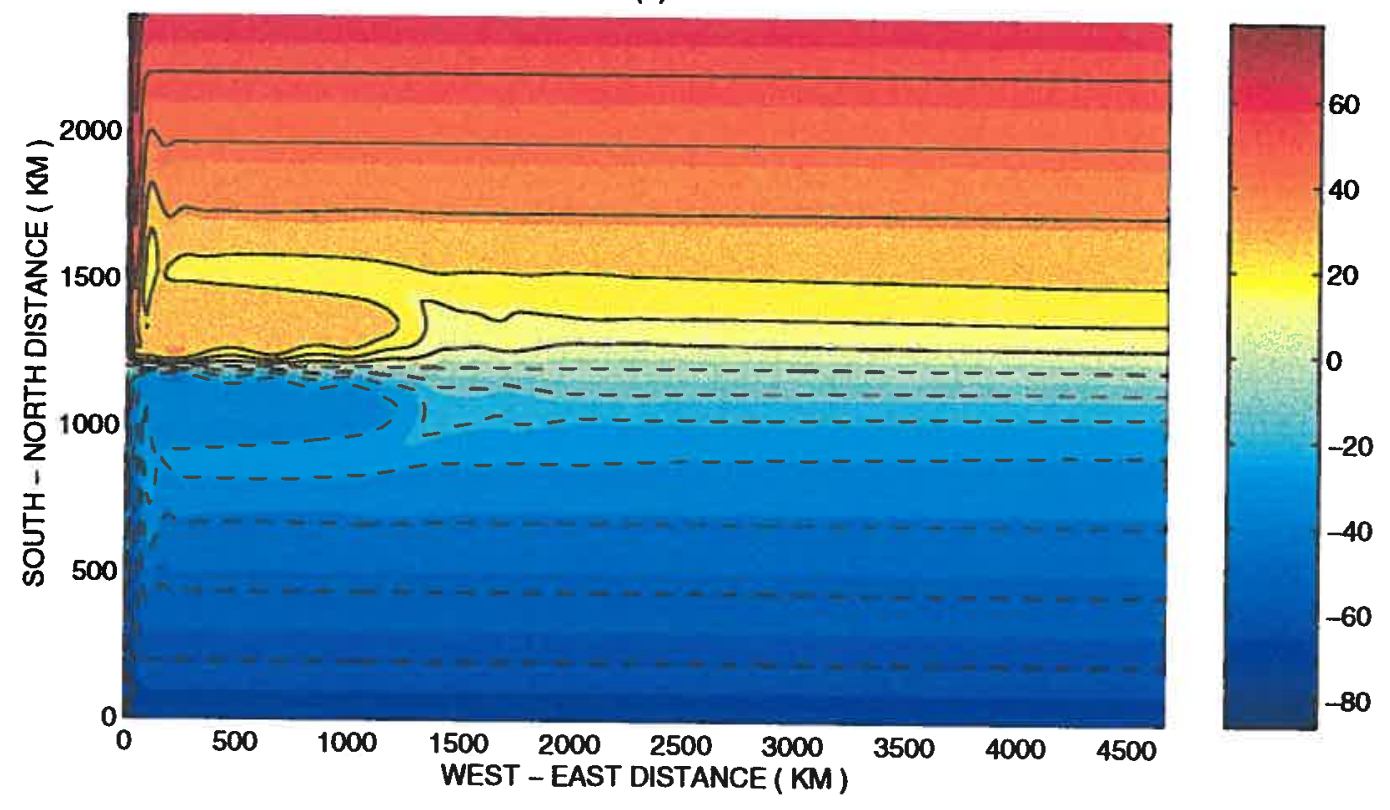

Figure 4-10: Contours of time mean streamfunction (a) and potential vorticity (b) for $\mathcal{A}_{2}=5.0 \cdot 10^{-8} \mathrm{sec}^{-1}$, initial inflow transport of $16 \mathrm{~Sv}$ and $\mathcal{A}_{6}=8 \cdot 10^{10} \mathrm{~m}^{4} \mathrm{sec}^{-1}$. Solid line is used for positive values, dashed line is used for negative values. 


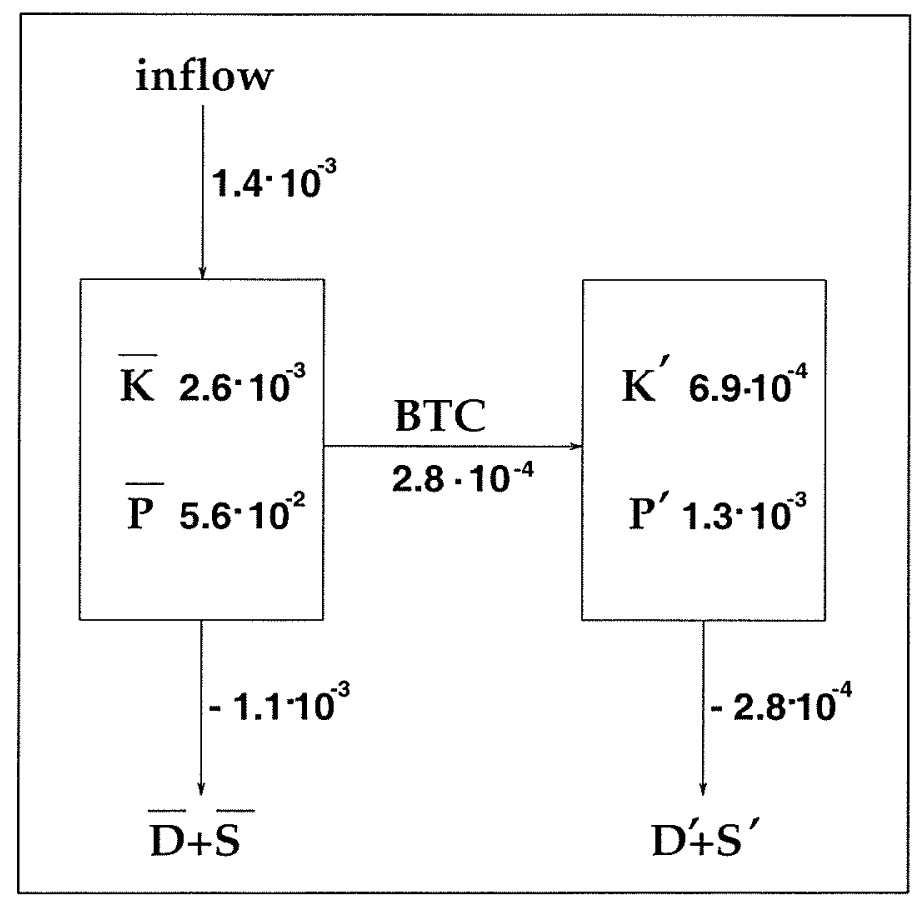

Figure 4-11: Energy diagram for $\mathcal{A}_{2}=5.0 \cdot 10^{-8} \mathrm{sec}^{-1}$, initial inflow transport of 16 Sv and $\mathcal{A}_{6}=8 \cdot 10^{10} \mathrm{~m}^{4} \mathrm{sec}^{-1}$.

\section{State $C$}

In agreement with linear stability analysis of section 4.2 .1 , the flow becomes unstable when the interfacial friction coefficient falls below some critical value. In state $C$, when $\mathcal{A}_{2}^{\text {dim }}$ is close to the critical value, the model trajectory is trapped in the vicinity of an antisymmetric fixed point, since the eddy-mean flow interactions dominate the eddy-eddy interactions. This explains why the model stays in one dynamical regime and why the time mean streamfunction and potential vorticity fields (Figure 4-10) closely resemble the steady antisymmetric solution. The flow structure consists of an eastward flowing jet and two recirculating gyres. The longitudinal size of the time mean recirculations equals $1500 \mathrm{~km}$ (compared to $1600 \mathrm{~km}$ in the case of a steady solution) and does not substantially change in time.

The energy conversion diagram summarizing the energetics of the model shows that the barotropic conversion term is small. The perturbation energy is only $3.3 \%$ of 
(a)

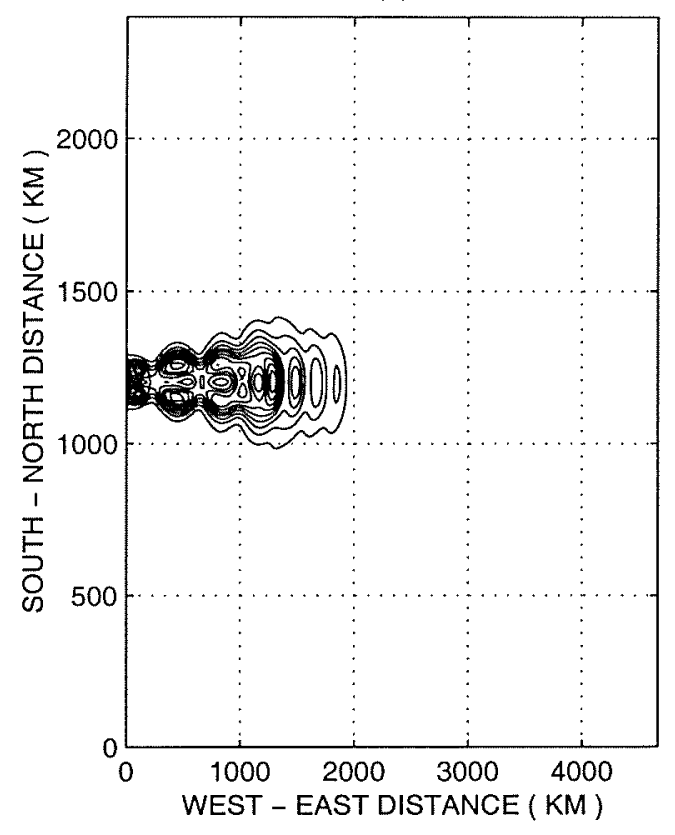

(c)

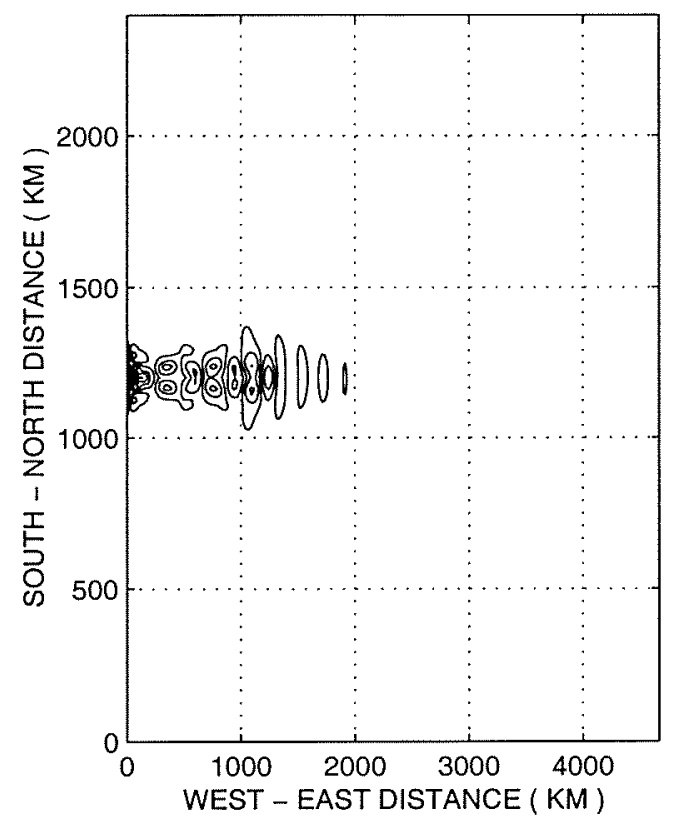

(b)

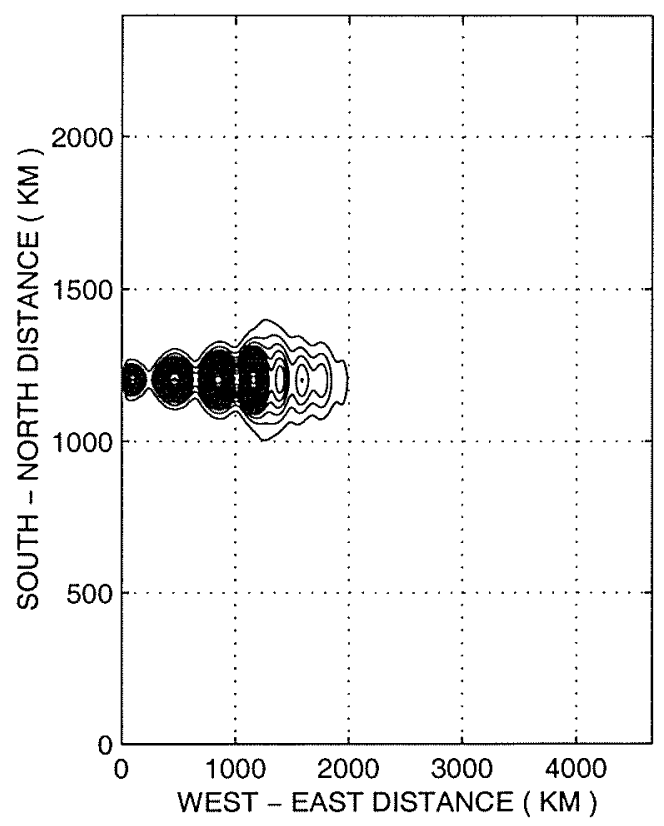

(d)

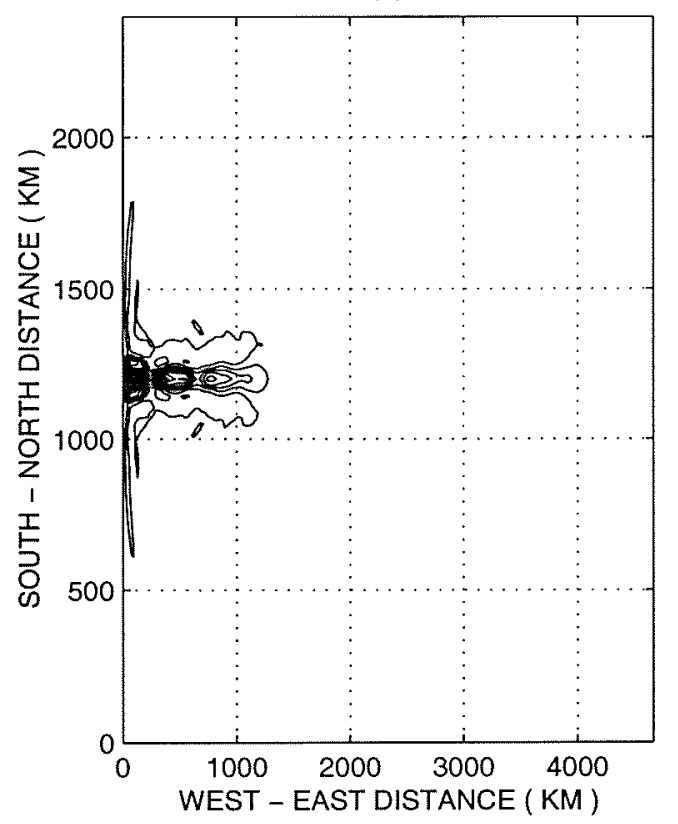

Figure 4-12: Time mean perturbation kinetic energy density (a), perturbation potential energy density (b), barotropic conversion energy density (c) and perturbation energy dissipation (d). Parameters: $T_{i n}=16 \mathrm{~Sv}, \mathcal{A}_{2}=5.0 \cdot 10^{-8} \mathrm{sec}^{-1}, \mathcal{A}_{6}=8 \cdot 10^{10}$ $\mathrm{m}^{4} \mathrm{sec}^{-1}$. 
the total energy. The eddy field is dominated by eastward and westward propagating meanders. Perturbation kinetic and potential energy densities (Figure 4-12) have spatial structures resembling the structure of the linearly unstable mode. Apparently, the dynamics is dominated by the linear eddy-mean flow interactions and weaklynonlinear eddy-eddy interactions. The amplitude of the perturbation fields near the separation point shows that the latter has weak meridional excursions due to meander movement. The dissipation of the perturbation energy occurs in the area of western boundary current and on the axis of the jet west of $1000 \mathrm{~km}$. The barotropic conversion energy density is aligned with the jet axis and is maximum west of 1500 $\mathrm{km}$. The spatial structure of the perturbation fields indicates that rings formed east of the recirculations are so weak that they are quickly dissipated during their westward propagation in the recirculation regions. Therefore, they mostly provide weak potential vorticity mixing across the front in the region east of the recirculations.

\section{State $D$}

In state $D$, the model exhibits low frequency variability associated with changes in the zonal extent of the recirculating gyres. The time mean streamfunction and potential vorticity fields (Figure 4-13) have $1200 \mathrm{~km}$ long recirculations. The energy level of the time mean streamfunction is $14 \%$ smaller than the energy level of the antisymmetric steady solution. During the flow evolution, the recirculation size fluctuates between 700 and almost $1800 \mathrm{~km}$. Since most of the potential energy is contained in the recirculation areas, and because the total energy is dominated by the potential energy, the time series of total energy shows periods of high and low values (Figure 4-14).

For high values of total energy, the model trajectory is in the vicinity of an antisymmetric steady solution. The recirculation gyres are about $1800 \mathrm{~km}$ long, and the eddy field is very weak. The flow however, is linearly unstable, and therefore growing disturbances draw energy from the mean flow. The amplitude of the eddy field increases with time, until nonlinear interactions start dominating. At this moment, the 
(a)

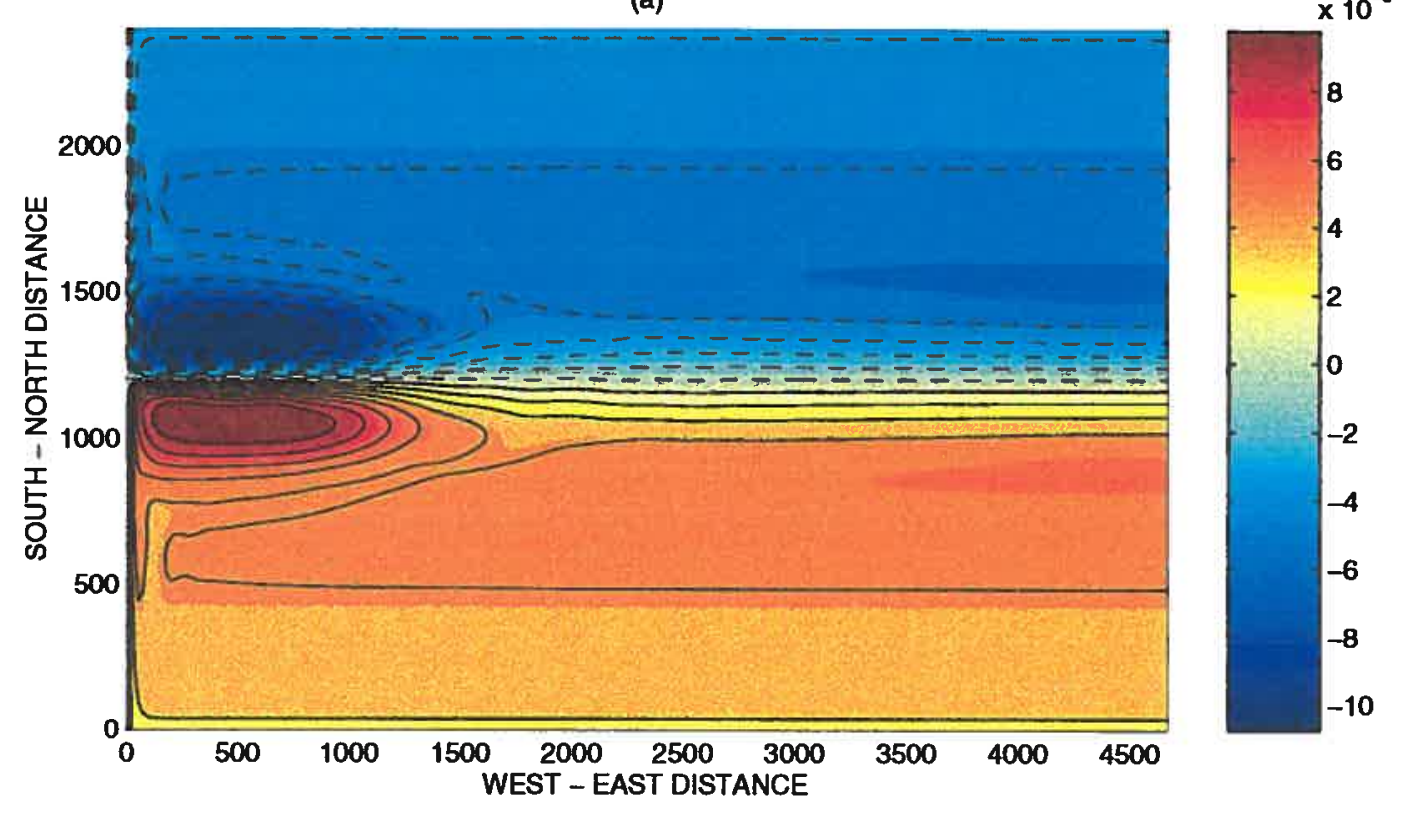

(b)

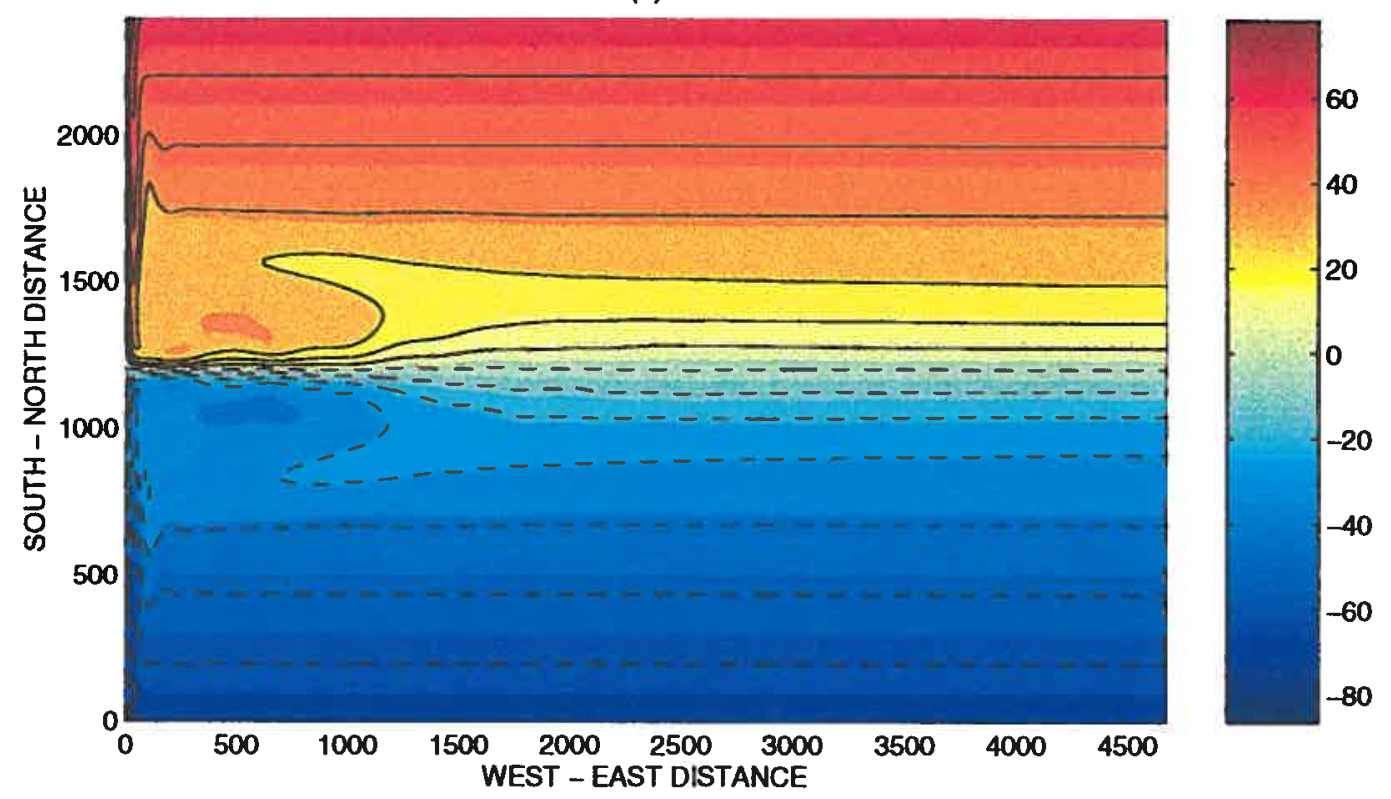

Figure 4-13: Contours of time mean streamfunction (a) and potential vorticity (b) for $\mathcal{A}_{2}=3.5 \cdot 10^{-8} \mathrm{sec}^{-1}$, initial inflow transport of $16 \mathrm{~Sv}$ and $\mathcal{A}_{6}=8 \cdot 10^{10} \mathrm{~m}^{4} \mathrm{sec}^{-1}$. Solid line is used for positive values, dashed line is used for negative values. 


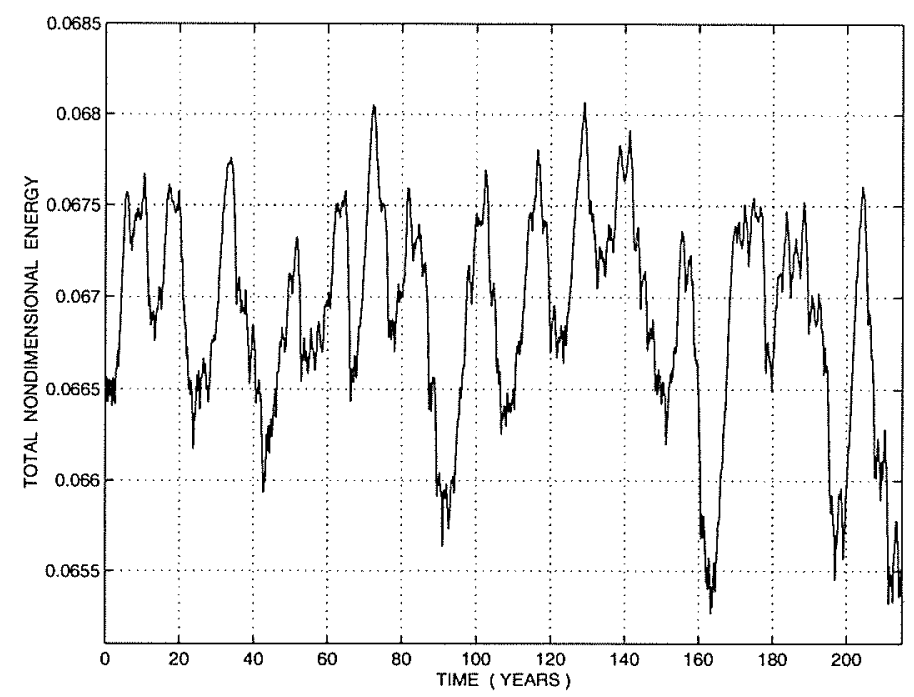

Figure 4-14: Time series of total nondimensional energy for $\mathcal{A}_{2}=3.5 \cdot 10^{-8} \mathrm{sec}^{-1}$, initial inflow transport of $16 \mathrm{~Sv}$ and $\mathcal{A}_{6}=8 \cdot 10^{10} \mathrm{~m}^{4} \mathrm{sec}^{-1}$.

meanders reach large amplitudes, which leads to the formation of stronger rings east of the recirculations. As the rings travel westward in the recirculation region, they mix opposite sign vorticity into the surrounding fluid and homogenize the surrounding, thus decreasing the recirculation strength. Upon reaching the western wall, they are advected by the western boundary current and entrained into the eastward jet. The formation and propagation of strong meanders and rings leads to a decrease in the size of the recirculating gyres and to a subsequent drop in the total energy level. Once the recirculations shrink, the flow becomes less turbulent, and perturbations decay. At this point, the advection of potential vorticity by the western boundary current becomes dominant and restores large recirculations, which causes the total energy to increase.

The conical shape of perturbation kinetic and potential density fields (Figure 4-15) is connected to the existence of meanders and rings not only on the axis of the jet, but also in the recirculation regions and along the western wall. The barotropic conversion density and the dissipation of perturbation energy are maximum on the axis of the jet west of $500 \mathrm{~km}$ and along the western wall. 
(a)

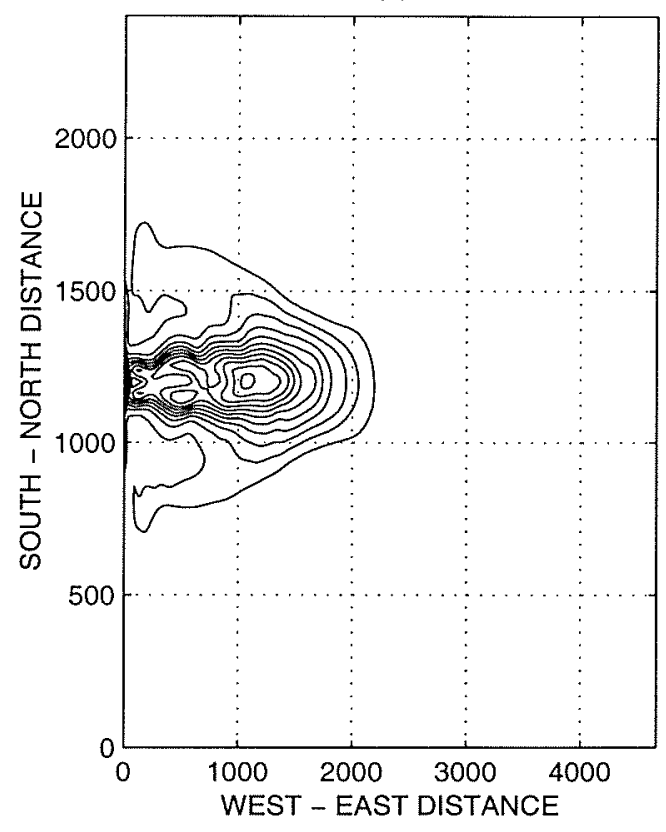

(c)

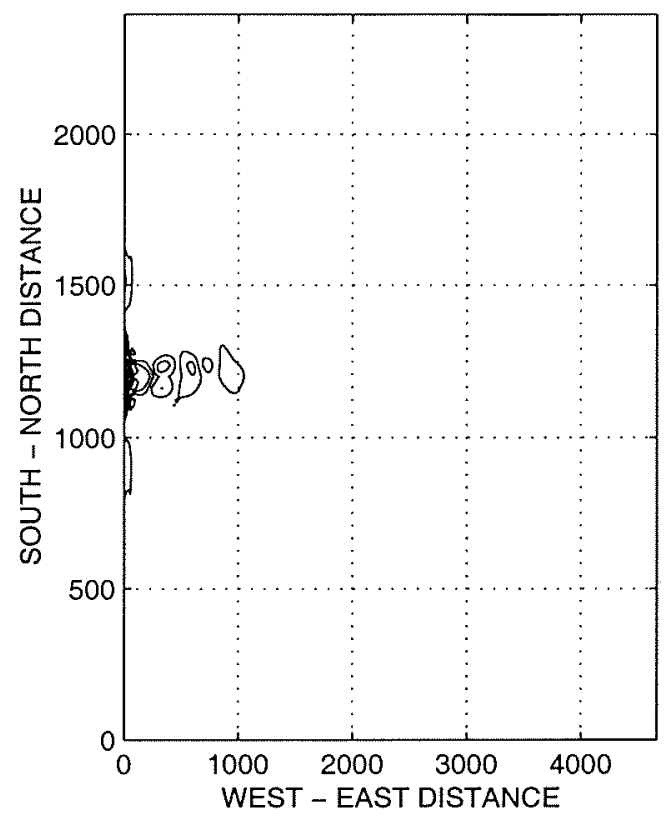

(b)

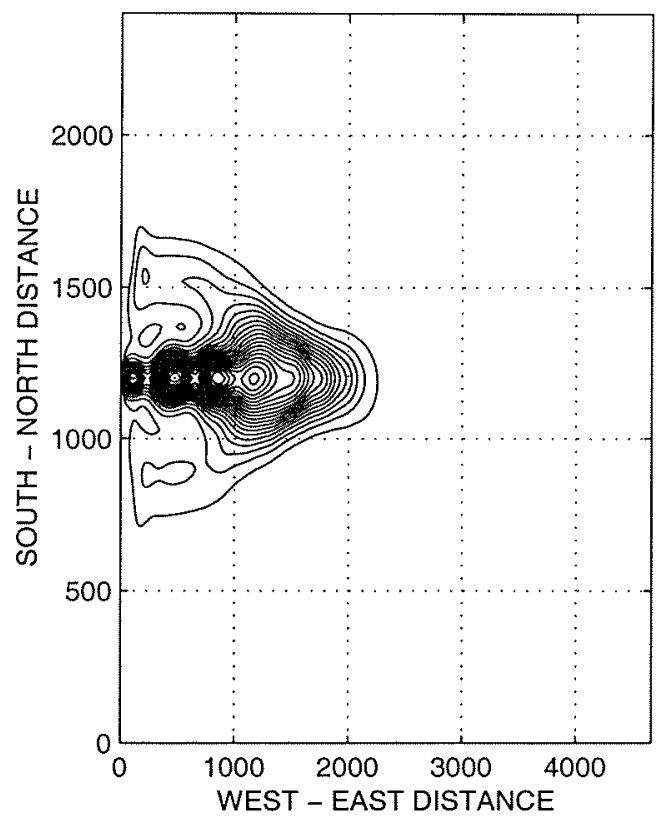

(d)

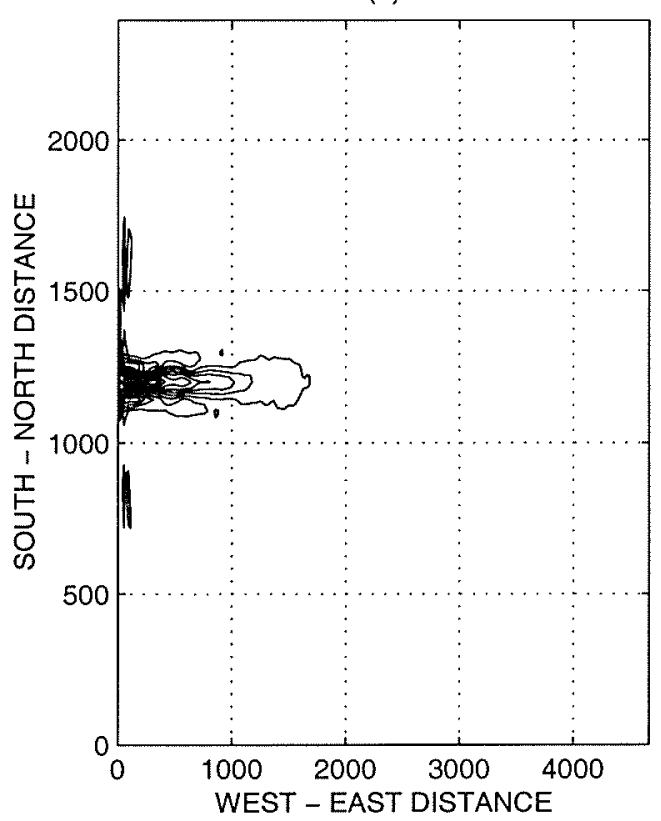

Figure 4-15: Time mean perturbation kinetic energy density (a), perturbation potential energy density (b), barotropic conversion energy density (c) and perturbation energy dissipation (d). Parameters: $T_{i n}=16 \mathrm{~Sv}, \mathcal{A}_{2}=3.5 \cdot 10^{-8} \mathrm{sec}^{-1}, \mathcal{A}_{6}=8 \cdot 10^{10}$ $\mathrm{m}^{4} \mathrm{sec}^{-1}$. 


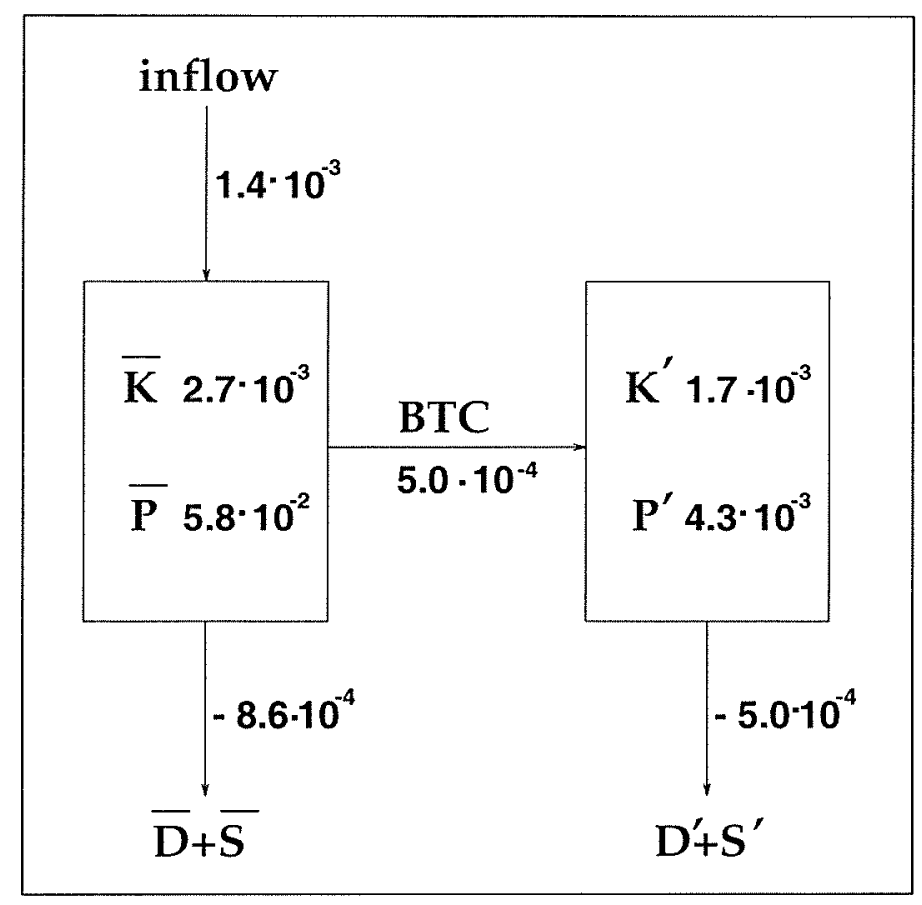

Figure 4-16: Energy diagram for $\mathcal{A}_{2}=3.5 \cdot 10^{-8} \mathrm{sec}^{-1}$, initial inflow transport of 16 $\mathrm{Sv}$ and $\mathcal{A}_{6}=8 \cdot 10^{10} \mathrm{~m}^{4} \mathrm{sec}^{-1}$.

The energy diagram (Figure 4-16) summarizes the domain-integrated energy transfers for state $D$. Compared to state $C$, the energy associated with the mean field did not change much. However, the perturbation kinetic energy was an order of magnitude larger, and the perturbation potential energy grew more than three times. The barotropic conversion was almost twice as large. Since the interfacial friction was weaker, both mean and perturbation energy dissipation terms were smaller than in state $C$. The partition of perturbation energy reached $9 \%$ compared to only $3 \%$ in state $C$.

\section{State $E$}

In state $E$, the model exhibits strong low frequency variability associated with high energy periods, when the flow structure is as in state $D$, and with low energy periods, when the flow structure is as in state $F$. The details of the dynamics for state $E$ are presented in section 4.4 . 


\section{State $F$}

For small values of the interfacial friction coefficient, the flow is strongly unstable. After perturbing an antisymmetric steady solution (see section 4.2.1), the eddy field grows rapidly, drastically changing the structure of the mean flow and causing the recirculations to disappear. The separation point of the eastward jet suffers large meridional excursions. Since northward and southward overshoots are equally possible, the western boundary currents appear to be separating early from the wall and flowing eastward, thus creating a region of zero streamfunction and "homogenized"

potential vorticity (Figure 4-17). The time mean fields are therefore misleading and can not be interpreted as structures persisting in time. The recirculations are not present in the instantaneous fields. Instead, there are individual vortices, trapped between meander's highs and lows, which provide weak mixing on the flanks of the jet.

The strong eddy field is dominated by large amplitude, westward propagating meanders, which do not allow any ring formation in the interior of the model domain. However, the interaction of these meanders with the western wall creates large excursions in the separation point and strong rings that, due to a no-normal-flow boundary condition, move along the western boundary against the advective force of the western boundary current. Boundary ring formation provides strong potential vorticity mixing across the front and tends to decrease the asymmetry in the inflow conditions near the collision point. However, for a given value of the interfacial friction coefficient, the meander amplitudes are large enough to guarantee strong excursions in the separation point, which keeps the model trajectory in a low energy state.

In contrast with states $C$ and $D$, the spatial structure of perturbation energy, its dissipation and barotropic conversion (Figure 4-18) have maxima along the western boundary. Their penetration into the model interior is less than $500 \mathrm{~km}$. The energy diagram (Figure 4-19) summarizes domain-integrated energy transfers. 
(a)

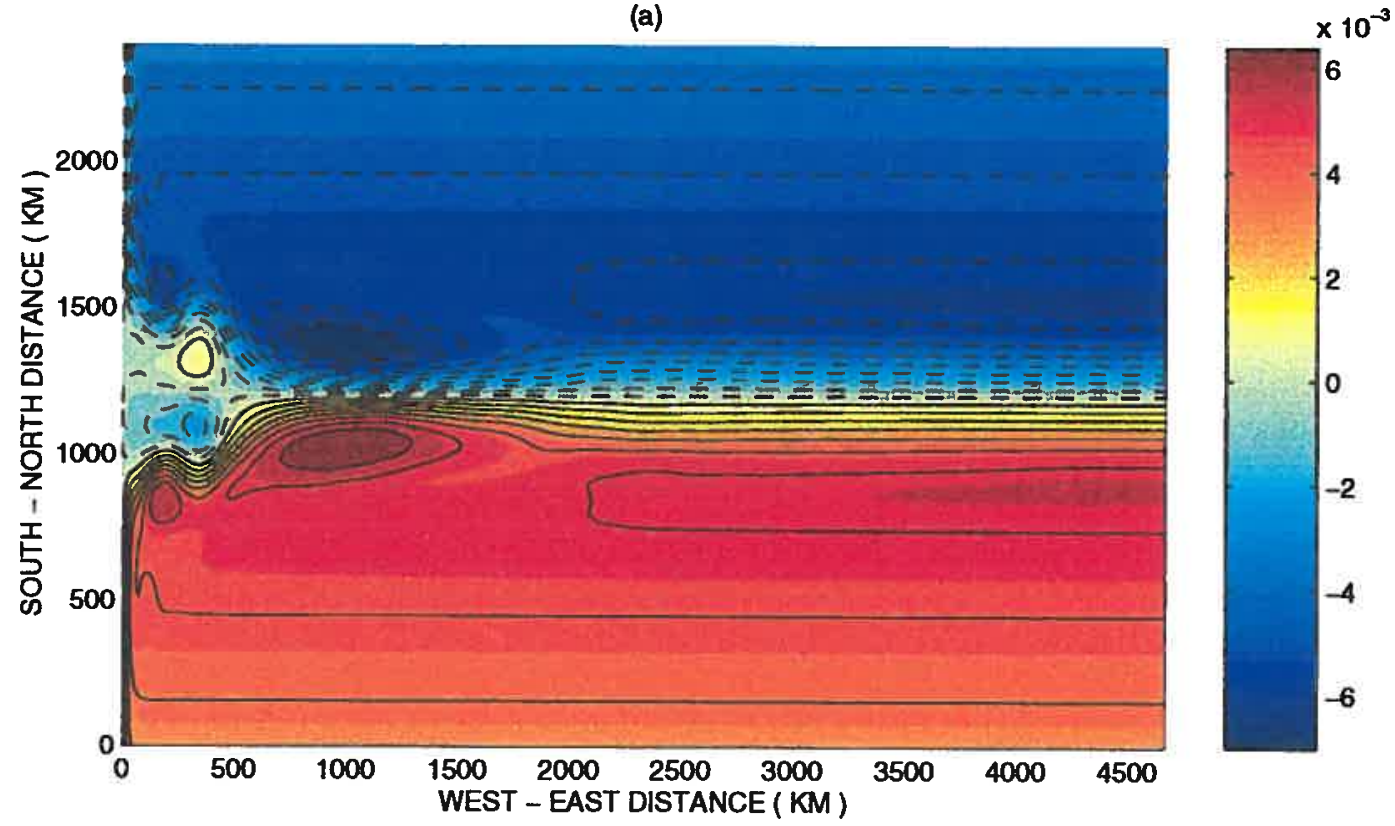

(b)

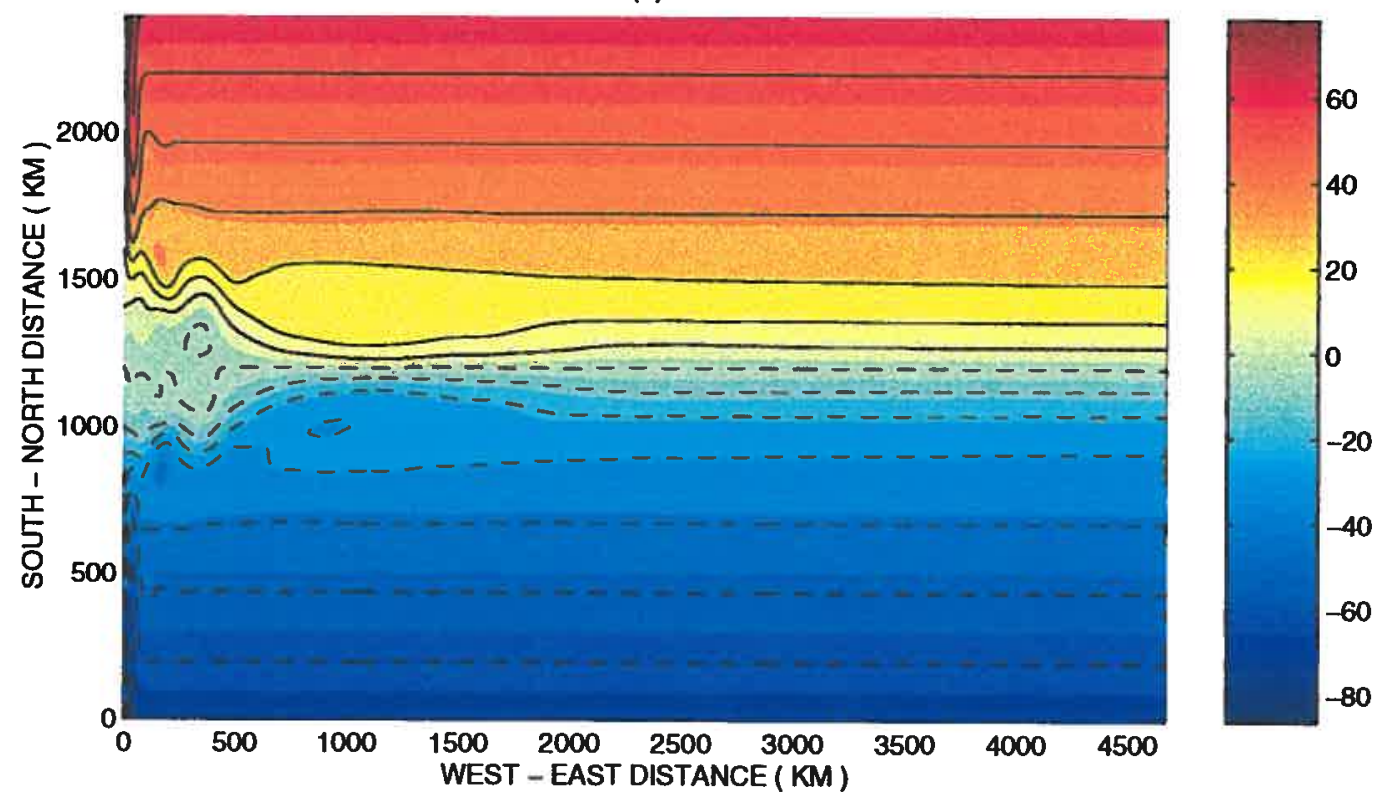

Figure 4-17: Contours of time mean streamfunction (a) and potential vorticity (b) for $\mathcal{A}_{2}=2.0 \cdot 10^{-8} \mathrm{sec}^{-1}$, initial inflow transport of $16 \mathrm{~Sv}$ and $\mathcal{A}_{6}=8 \cdot 10^{10} \mathrm{~m}^{4} \mathrm{sec}^{-1}$. Solid line is used for positive values, dashed line is used for negative values. 
(a)

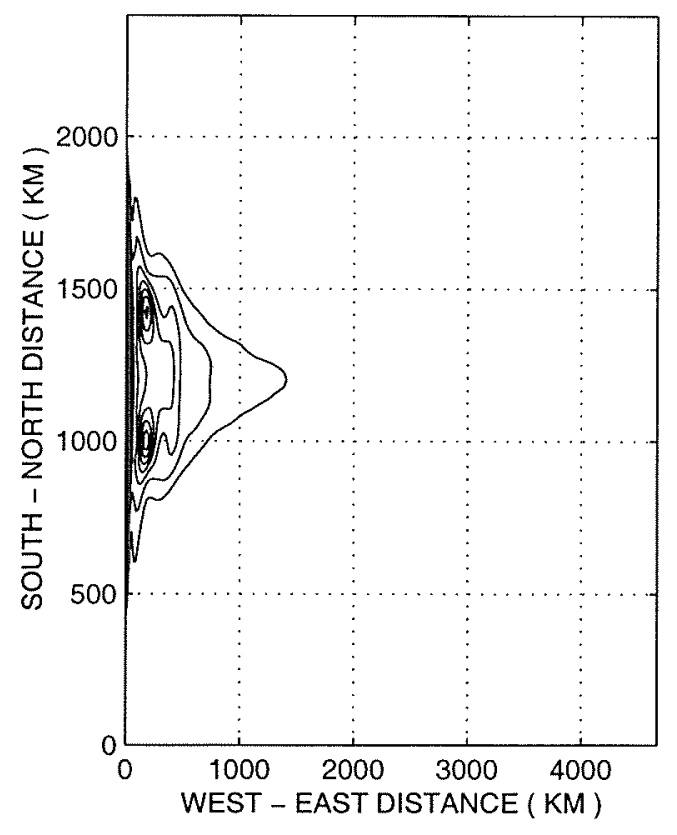

(c)

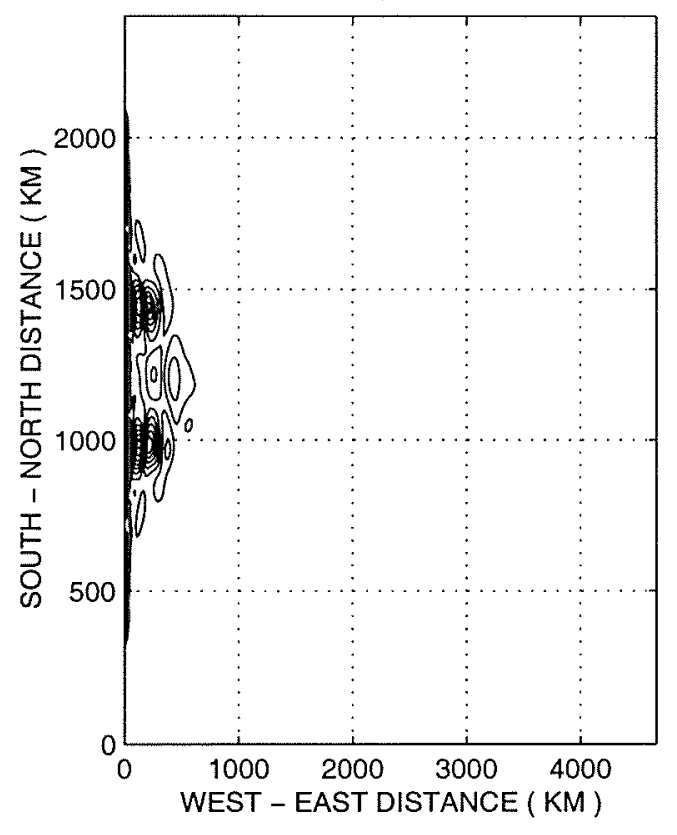

(b)

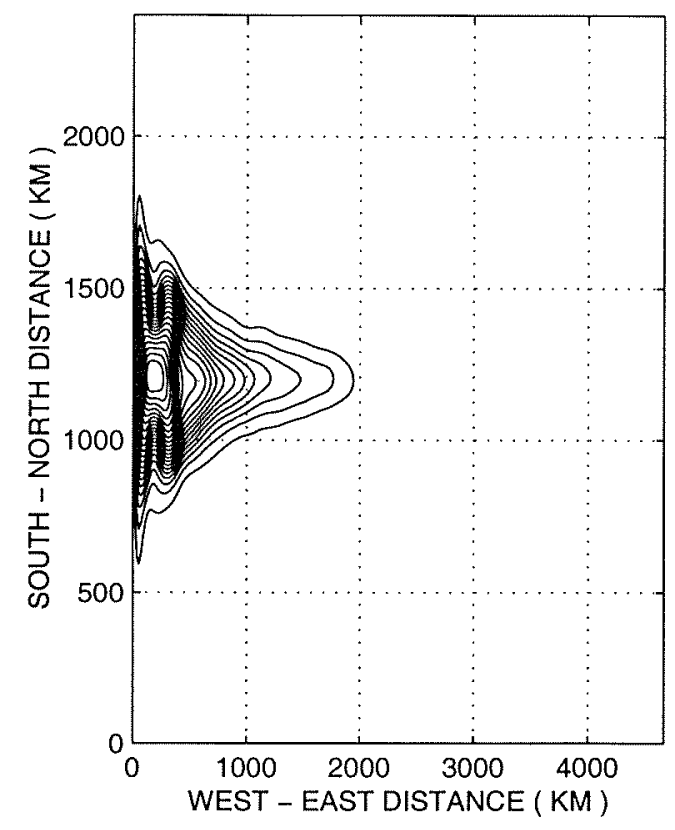

(d)

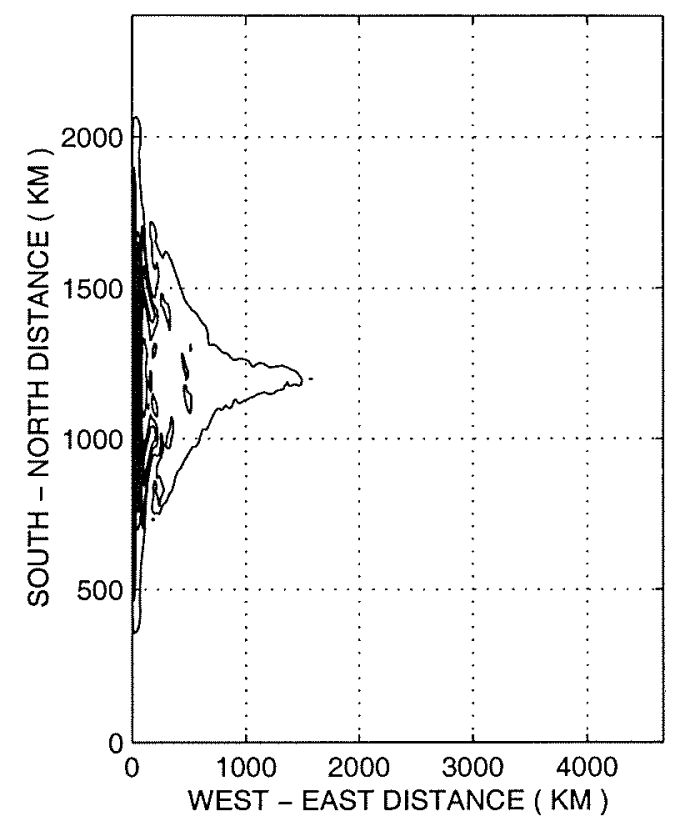

Figure 4-18: Time mean perturbation kinetic energy density (a), perturbation potential energy density (b), barotropic conversion energy density (c) and perturbation energy dissipation (d). Parameters: $T_{i n}=16 \mathrm{~Sv}, \mathcal{A}_{2}=2.0 \cdot 10^{-8} \mathrm{sec}^{-1}, \mathcal{A}_{6}=8 \cdot 10^{10}$ $\mathrm{m}^{4} \mathrm{sec}^{-1}$. 


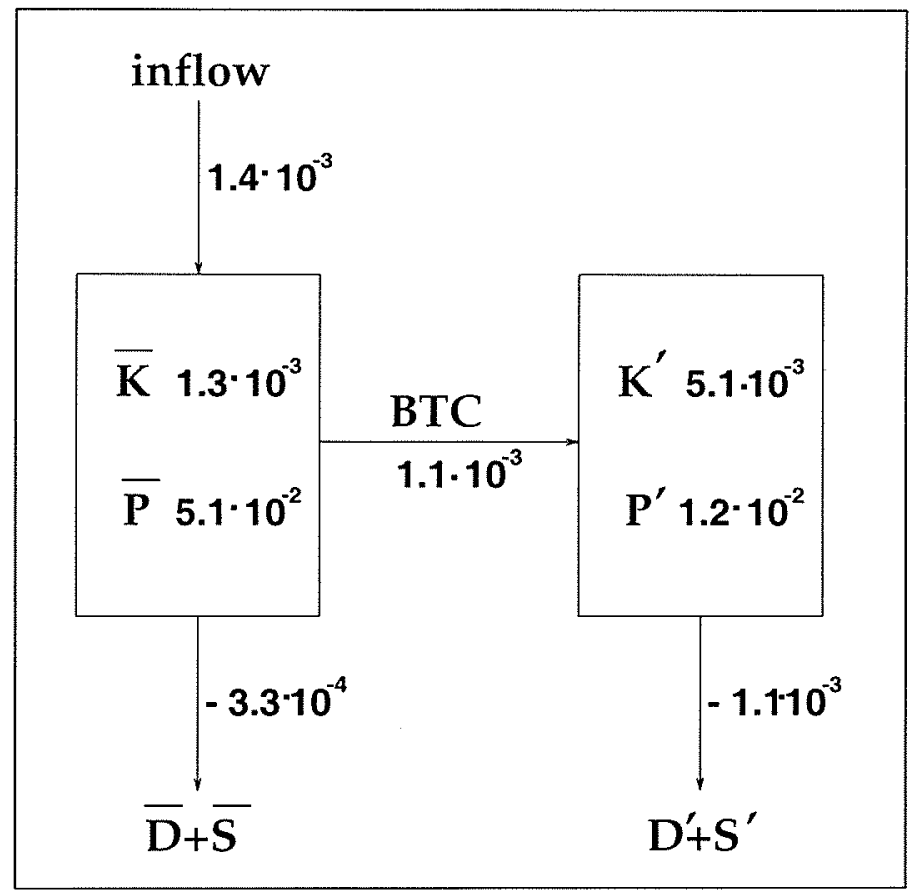

Figure 4-19: Energy diagram for $\mathcal{A}_{2}=2.0 \cdot 10^{-8} \mathrm{sec}^{-1}$, initial inflow transport of 16 Sv and $\mathcal{A}_{6}=8 \cdot 10^{10} \mathrm{~m}^{4} \mathrm{sec}^{-1}$.

\subsection{Reference Run: State $E$}

\subsubsection{Dimensional Parameters}

As a reference case, we chose a run that displayed well-developed multiple dynamical regimes. The dimensional scales and parameters were chosen as follows:

$$
\begin{gathered}
L=2400 \mathrm{~km}, \\
L_{x}=4667 \mathrm{~km}, \\
L_{d e f}=47.64 \mathrm{~km}, \\
\mathcal{A}_{6}^{d i m}=8 \cdot 10^{10} \mathrm{~m}^{4} \mathrm{sec}^{-1}, \\
\mathcal{A}_{2}^{d i m}=3 \cdot 10^{-8} \mathrm{sec}^{-1}, \\
D_{1}=600 \mathrm{~m},
\end{gathered}
$$




$$
U=1 \mathrm{~m} \mathrm{sec}^{-1}
$$

Note that all the dimensional parameters are as in McCalpin and Haidvogel [25], except for the value of the interfacial friction coefficient, where they used $\mathcal{A}_{2}^{\text {dim }}=$ $10^{-7} \mathrm{sec}^{-1}$. With the above values of dimensional parameters, the strength of the total inflow into the model domain is estimated at $T_{i n}=16 \mathrm{~Sv}$. After the initial spin up, the model was integrated for 970 years to obtain all the necessary statistics. The dimensional time step was 140 minutes, and the streamfunction fields were recorded every 4.7 days.

\subsubsection{Energy Time Series and Their Spectra}

Figure 4-20 shows the time series of kinetic, potential and total energy which are characterized by chaotic fluctuations. On average, total energy is partitioned between $92 \%$ potential and $8 \%$ kinetic energy. Therefore, total and potential energy time series are well correlated, and we shall concentrate on the total energy behavior in the next sections.

The kinetic energy power density spectrum (Figure 4-21a) is red and has different slopes for periods less than 4 months and periods between 1 and 7 years. There are peaks in the spectrum at the time scales of 5.1 months and 7.5 months. To ensure their significance, we narrowed confidence intervals by computing power density spectrum for periods less than 5 years (Figure 4-23b). The energy associated with the 5-month period is at least twice as high as the energy of the 7-month period (Figure 4-23d). The first peak is associated with the interaction of large amplitude, westward propagating meanders with the western boundary during the low energy period, which leads to large meridional excursions in the separation point and to formation of rings on the wall. Figure 4-21b presents power density spectrum multiplied by the frequency which reveals that most of kinetic energy lies in the band between 1 


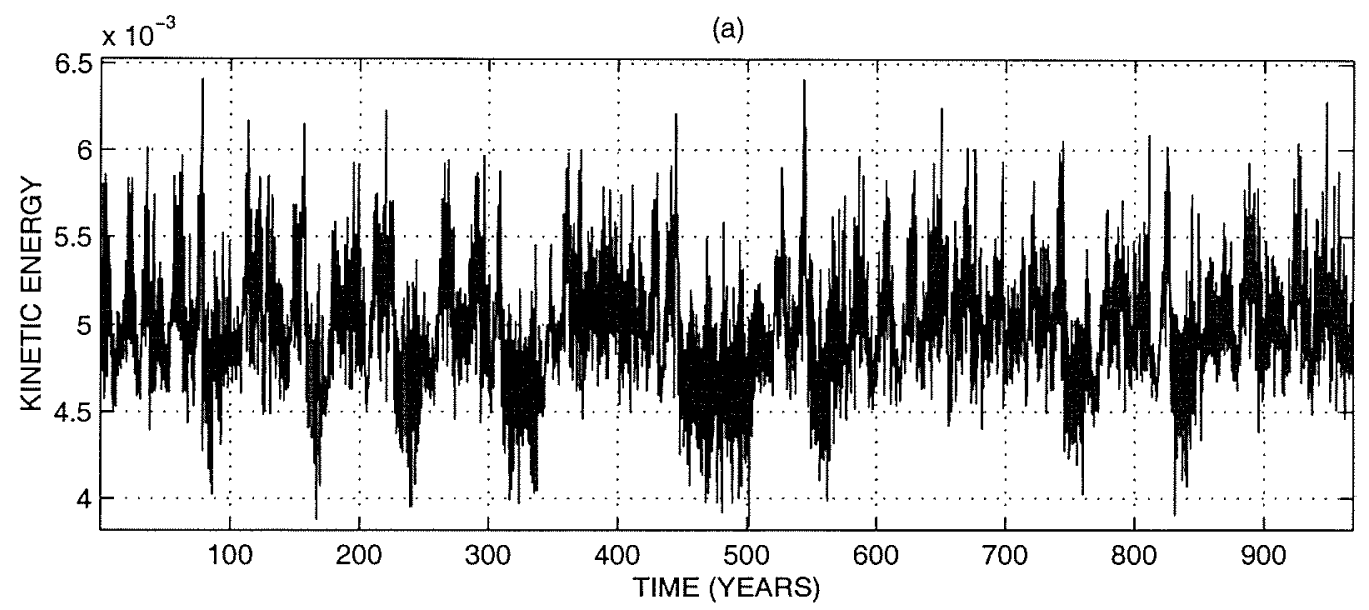

(b)

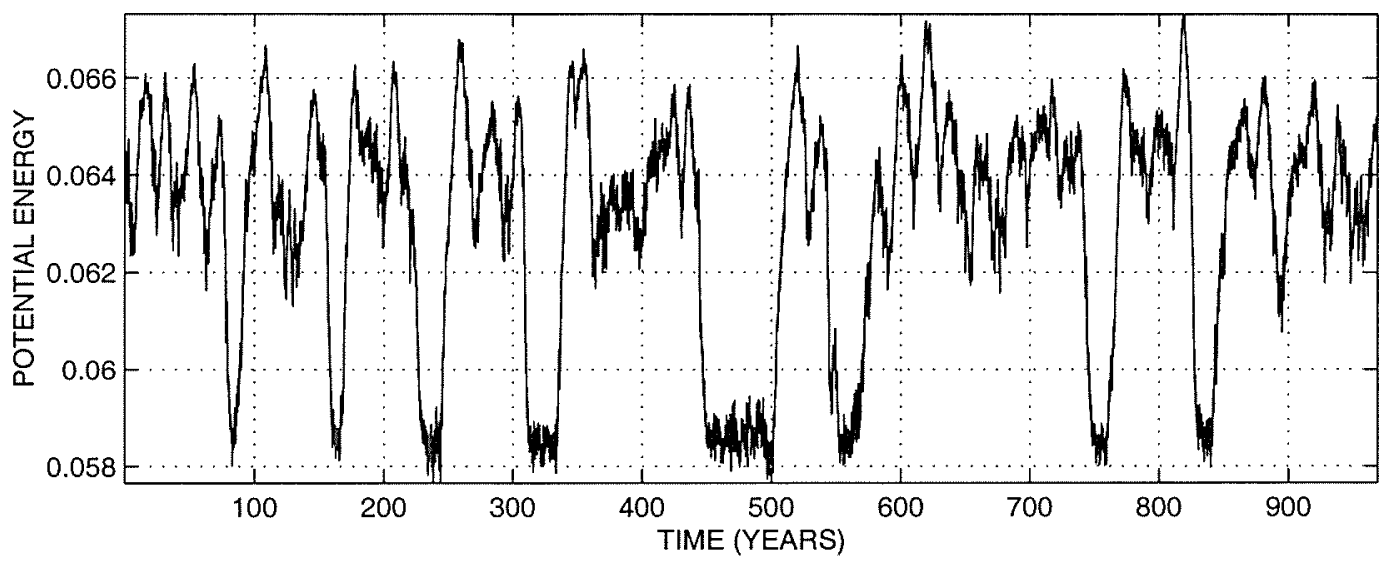

(c)

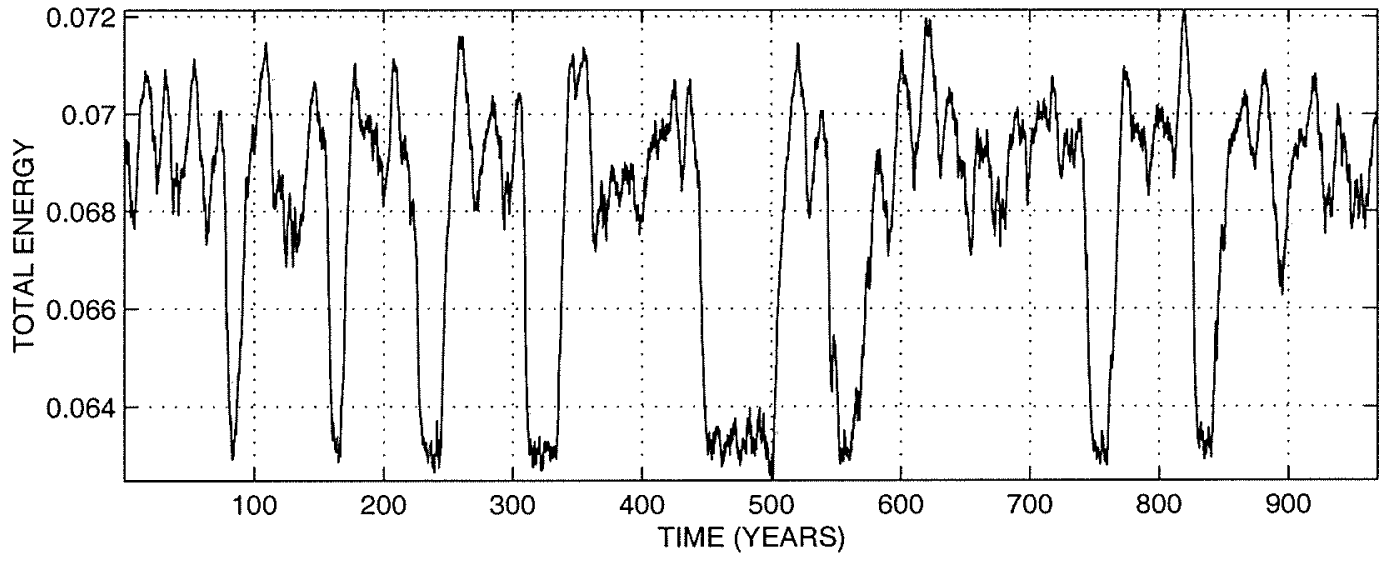

Figure 4-20: Time series of nondimensional kinetic (a), potential (b) and total (c) energy. Parameters: $T_{\text {in }}=16 \mathrm{~Sv}, \mathcal{A}_{6}^{d i m}=8 \cdot 10^{10} \mathrm{~m}^{4} \mathrm{sec}^{-1}$ and $\mathcal{A}_{2}^{d i m}=3.0 \cdot 10^{-8} \mathrm{sec}^{-1}$. 
(a)

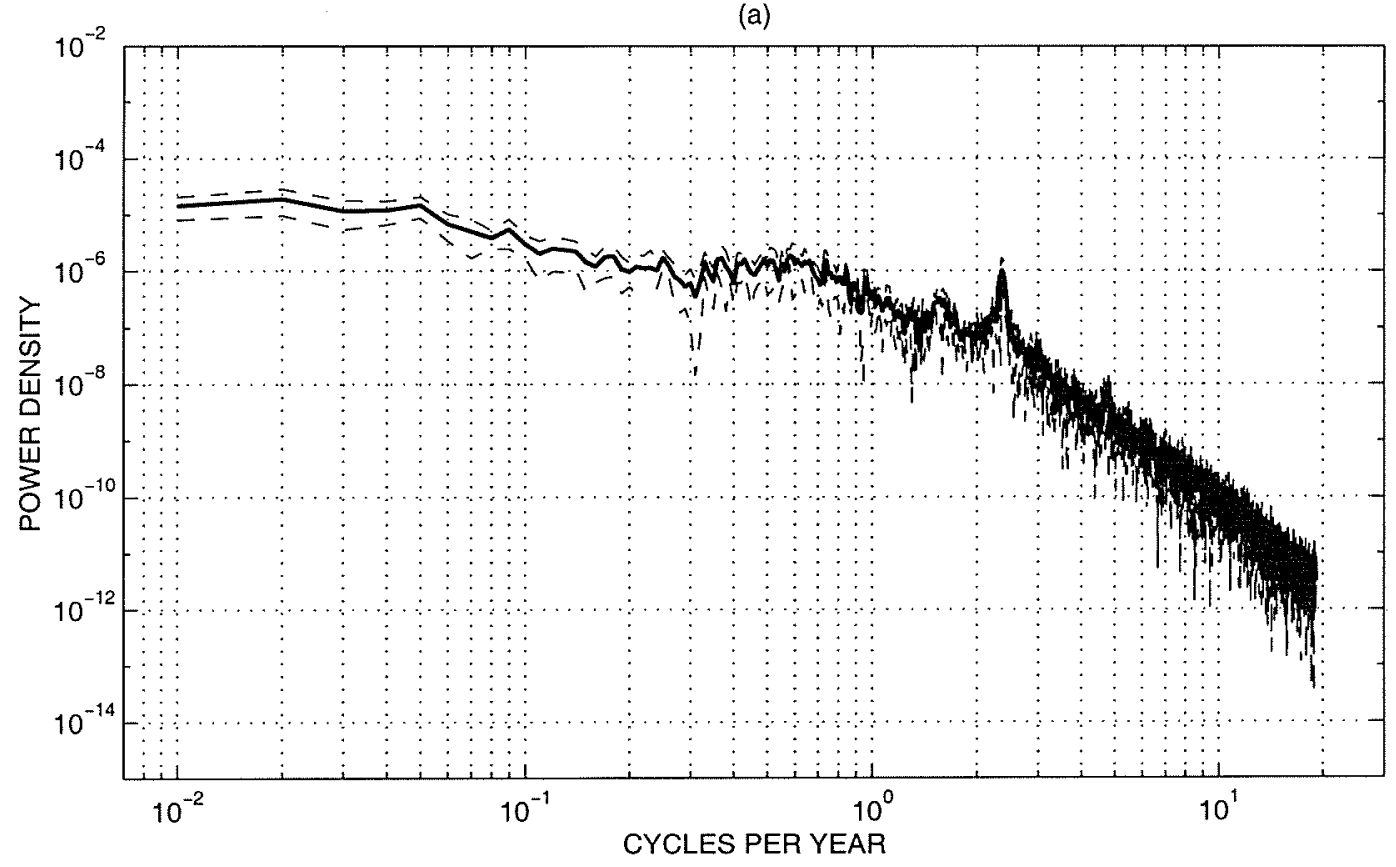

(b)

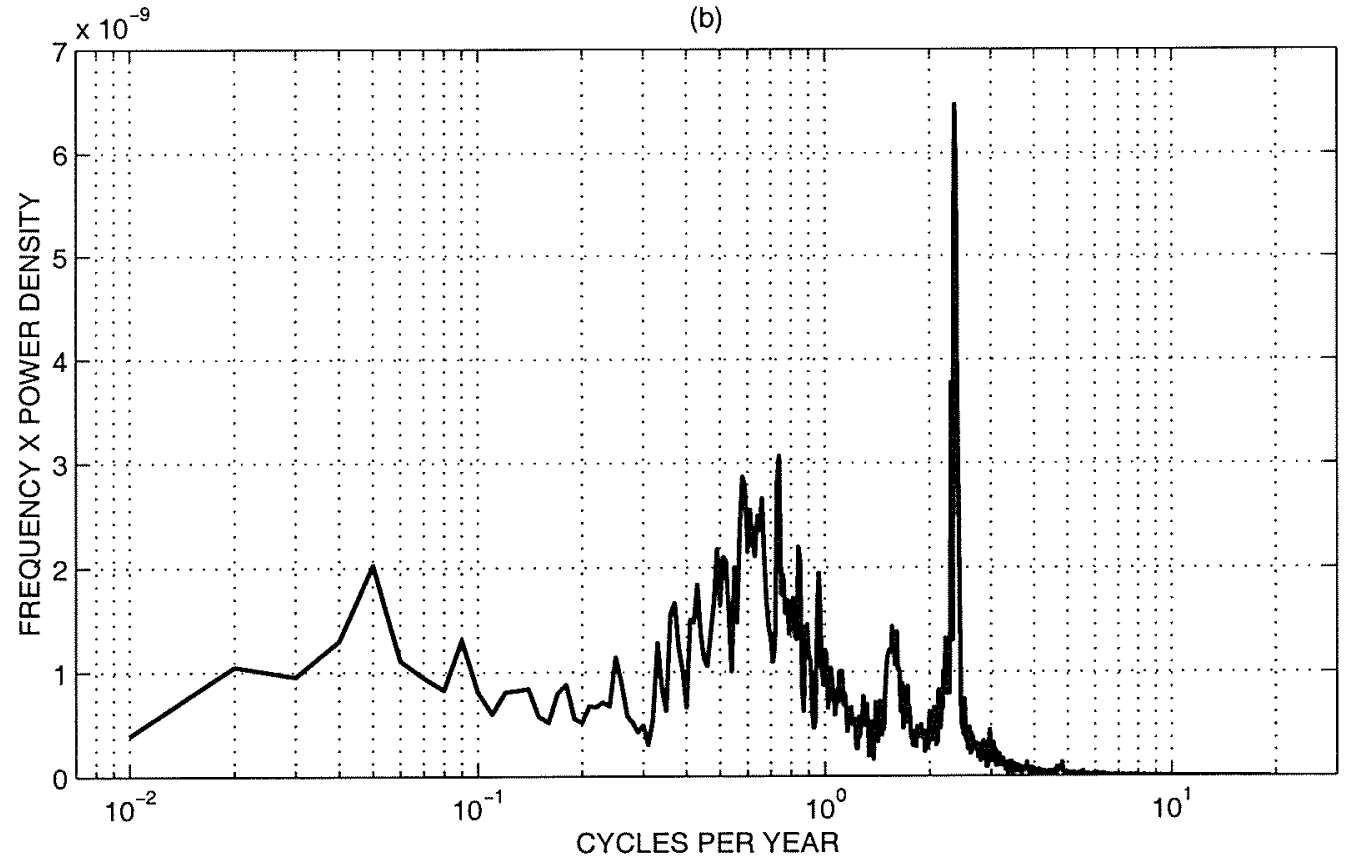

Figure 4-21: (a): Kinetic energy power density spectrum. Dashed line denotes confidence interval at $95 \%$. (b): Frequency times power density spectrum for kinetic energy. Parameters: $T_{i n}=16 \mathrm{~Sv}, \mathcal{A}_{6}^{\text {dim }}=8 \cdot 10^{10} \mathrm{~m}^{4} \mathrm{sec}^{-1}$ and $\mathcal{A}_{2}^{\text {dim }}=3.0 \cdot 10^{-8} \mathrm{sec}^{-1}$. 
(a)
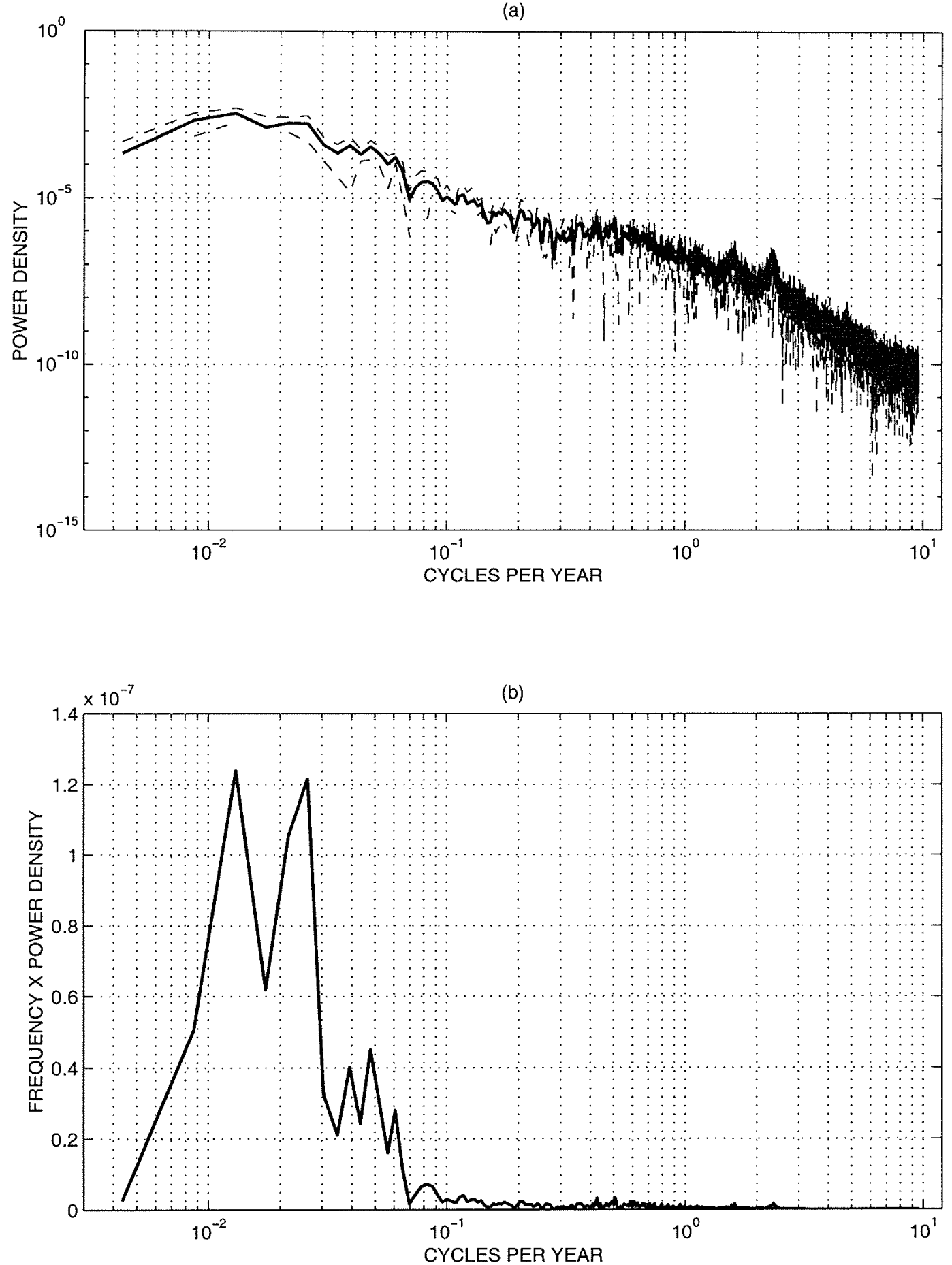

Figure 4-22: (a): Potential energy power density spectrum. Dashed line denotes confidence interval at $95 \%$. (b): Frequency times power density spectrum for potential energy. Parameters: $T_{\text {in }}=16 \mathrm{~Sv}, \mathcal{A}_{6}^{\text {dim }}=8 \cdot 10^{10} \mathrm{~m}^{4} \mathrm{sec}^{-1}$ and $\mathcal{A}_{2}^{\text {dim }}=3.0 \cdot 10^{-8} \mathrm{sec}^{-1}$. 

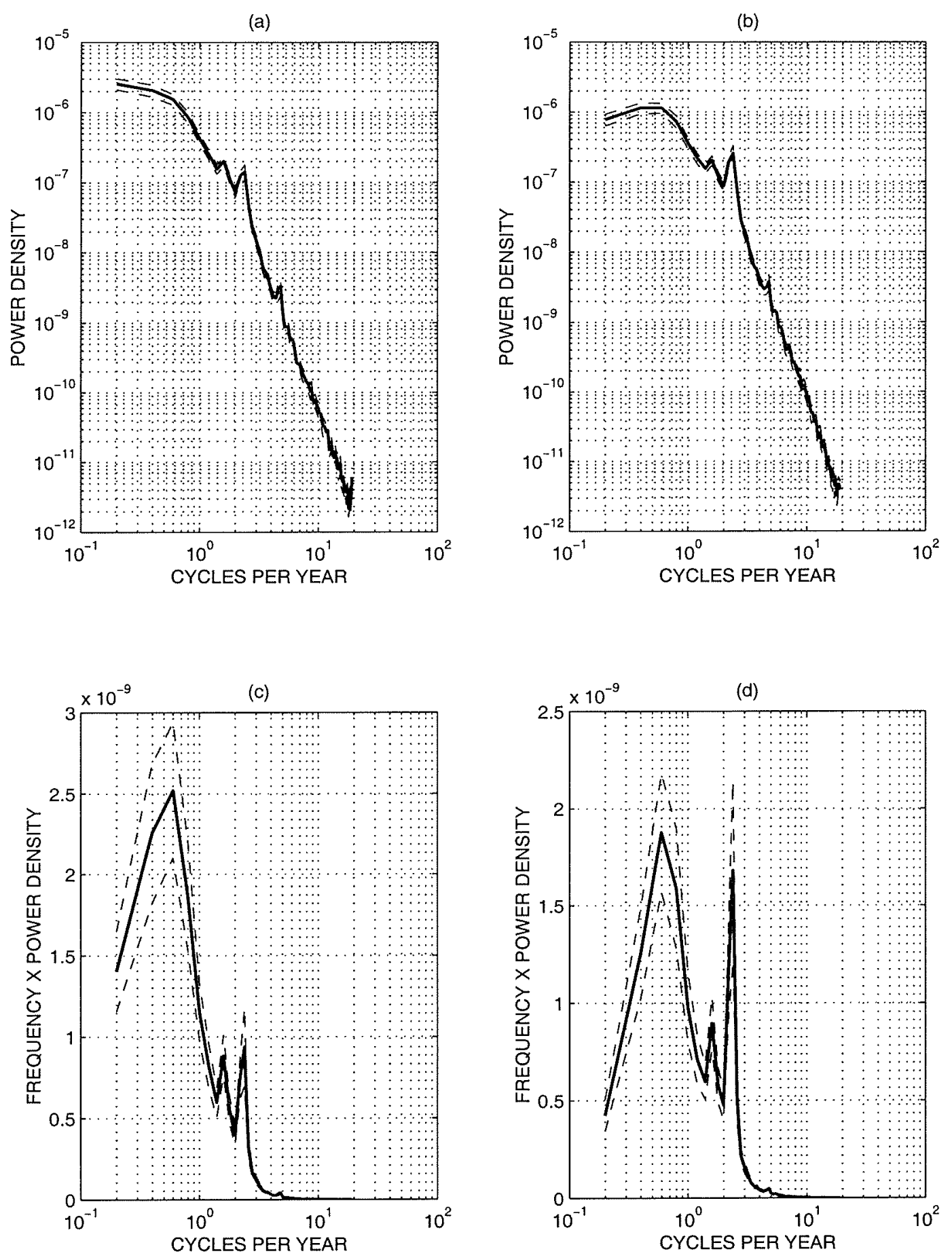

Figure 4-23: Potential (a) and kinetic (b) energy power density spectrum for periods less than 5 years. Corresponding frequency times power density spectrum for potential (c) and kinetic (d) energy. Dashed line denotes confidence interval at $95 \%$. Parameters: $T_{\text {in }}=16 \mathrm{~Sv}, \mathcal{A}_{6}^{\text {dim }}=8 \cdot 10^{10} \mathrm{~m}^{4} \mathrm{sec}^{-1}$ and $\mathcal{A}_{2}^{\text {dim }}=3.0 \cdot 10^{-8} \mathrm{sec}^{-1}$. 
and 10 years. For periods higher than 7 years, the kinetic energy spectrum is roughly flat.

Figure 4-22 shows that most of the potential energy is on the decadal time scales, with a significant level on interannual scales. Similar to the kinetic energy spectrum, it has peaks at 5.1 and 7.5 months (Figure 4-23a), however, the energy associated with them is quite low (Figure 4-23c), which might be the signature of the ring life cycle. For comparison, a peak in the potential energy spectrum (not in the kinetic energy) at periods between 7 and 8 months was also observed in the winddriven reduced-gravity model of McCalpin and Haidvogel [25] and was attributed to the preferred time scale of eddy-mean flow interaction.

\subsubsection{Energy Histograms}

The histogram of nondimensional total energy, TE, (Figure 4-24) has a bimodal distribution which reveals two preferred energy regimes of the model: state $\mathbf{L}$ with

$0.062 \leq T E \leq 0.064$ and state $\mathbf{H}$ with $0.066 \leq T E \leq 0.072$. The transition states, HL (high to low) and LH (low to high), are characterized by the energy level $0.064 \leq T E \leq 0.066$. As we show in the following section, HL and $\mathbf{L H}$ transitions are dynamically different. Based on the histogram, the probability of the model staying in a particular energy regime is estimated as 0.79 for state $\mathbf{H}, 0.16$ for state $\mathbf{L}$ and 0.05 for the transitions.

For comparison, in the wind-driven reduced-gravity model of McCalpin and Haidvogel [25], the total energy histogram did not have a bimodal distribution. Instead, it displayed three energy peaks associated with three different dynamical regimes. There was significant overlap between the different states, which indicated that the model was spending as much time in transition as in any given state.

The distribution of kinetic energy is not normal. MATLAB QQPLOT routine applied to the kinetic energy time series shows the quantiles of the normalized 
(a)

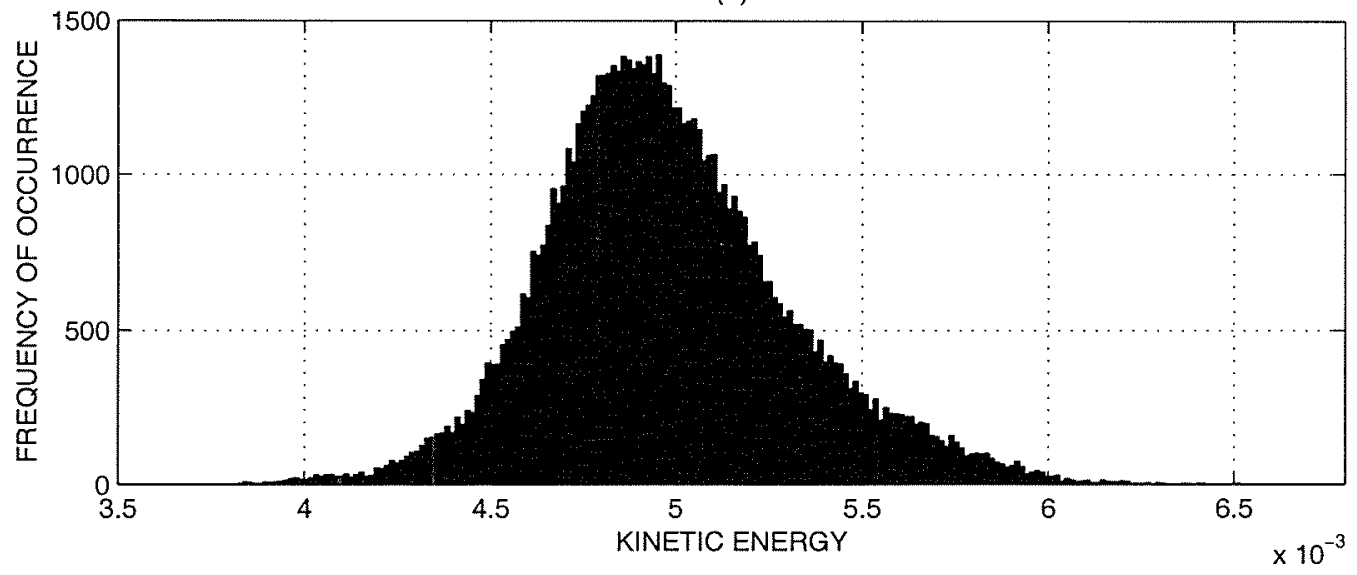

(b)

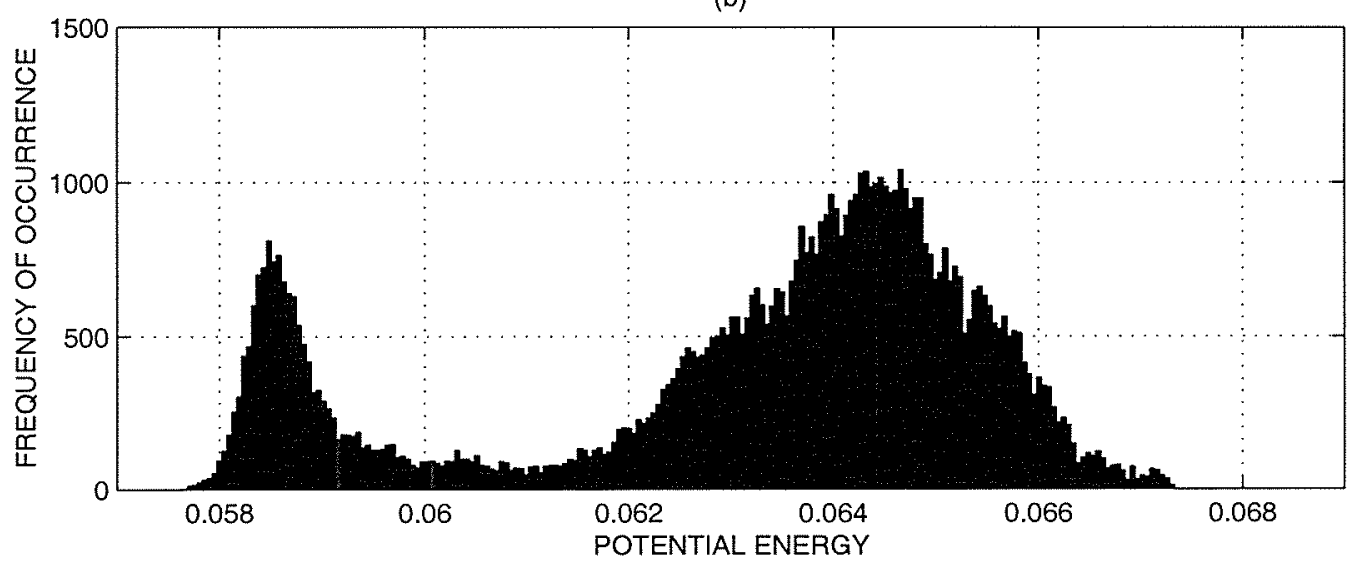

(c)

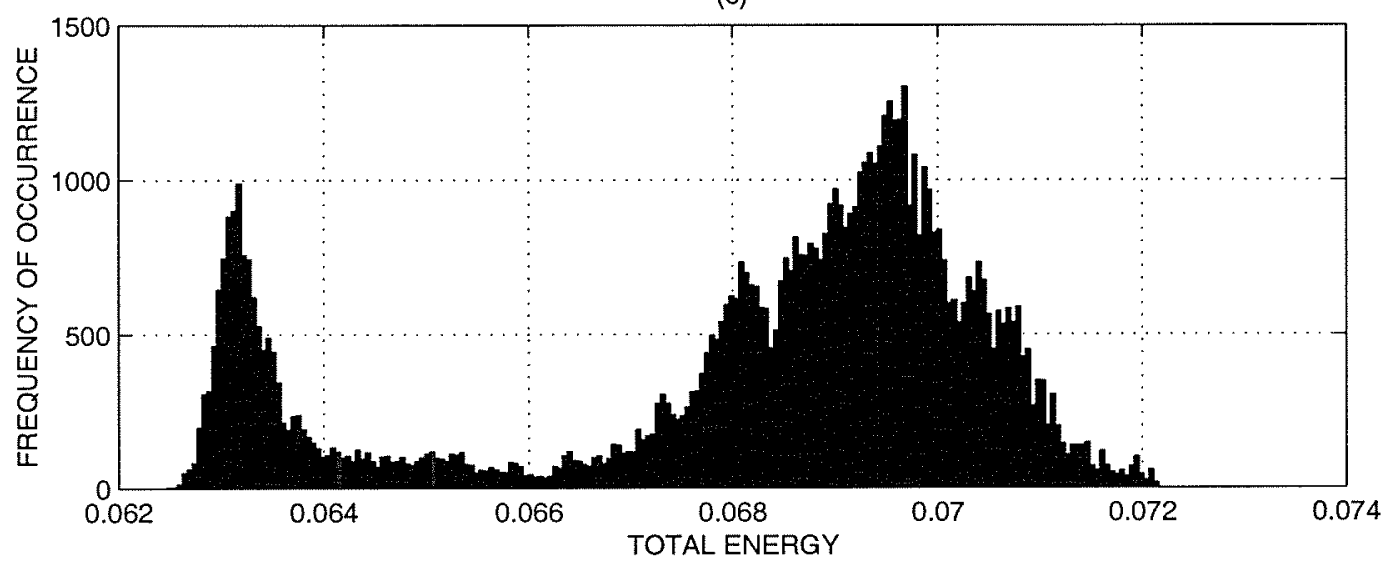

Figure 4-24: Histograms of kinetic (a), potential (b) and total (c) energy for the reference run. Parameters: $T_{i n}=16 \mathrm{~Sv}, \mathcal{A}_{6}=8 \cdot 10^{10} \mathrm{~m}^{4} \mathrm{sec}^{-1}$ and $\mathcal{A}_{2}=3.0$. $10^{-8} \mathrm{sec}^{-1}$. 


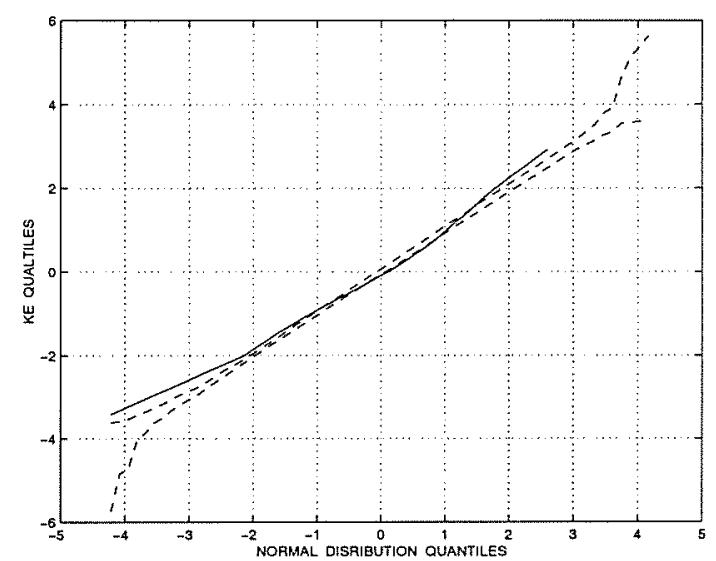

Figure 4-25: QQPLOT of total kinetic energy. Dashed line is error bars for normal distribution. Solid line denotes quantiles of the normalized kinetic energy time series vs quantiles of the generated time series with normal distribution. Parameters: $T_{i n}=$ $16 \mathrm{~Sv}, \mathcal{A}_{6}=8 \cdot 10^{10} \mathrm{~m}^{4} \mathrm{sec}^{-1}$ and $\mathcal{A}_{2}=3.0 \cdot 10^{-8} \mathrm{sec}^{-1}$.

kinetic energy time series, $\left\{K_{j=1}^{N}\right\}$, versus the quantiles of the generated random time series with normal distribution. Here, $K_{j}=\left(K E_{j}-\overline{K E}\right) / \operatorname{std}(K E)$, where $\overline{K E}=\frac{1}{N} \sum_{i=1}^{N} K E_{j}$ and $\operatorname{std}(K E)$ are the mean and the standard deviation of the kinetic energy time series, $\left\{K E_{j=1}^{N}\right\}$. QQPLOT allows to determine whether the time series come from the same distribution. Figure 4-25 indicates that the tails of the kinetic energy time series do not fit within the error bars calculated for normal distribution. Therefore, kinetic energy time series is not normal due to the existence of two different dynamical regimes. However, because of a significant overlap, the distribution does not appear to be bimodal.

As we describe later, in the present model, energy state $\mathbf{L}$ is related to a dynamical regime of an eastward flowing jet with strong meridional fluctuations in the position of the separation point and violent large-amplitude, westward propagating meanders. The state does not have recirculating gyres, and its eddy field is characterized by strong rings generated only on the western wall and not in the model interior. State $\mathbf{H}$, on the other hand, possesses well-developed recirculations. However, the longitudinal size and the symmetry of the recirculations suffer significant changes in time, thus giving rise to strong low frequency variability within energy 
state $\mathbf{H}$. The eddy field is characterized by periods of strong and weak ring formation in the region east of the recirculation gyres. An attempt to determine clear energetic boundaries between solutions with large, antisymmetric recirculations and small, asymmetric ones failed. Therefore, the energy histogram provides energetic boundaries only between a state with no recirculations and a state with spatially varying recirculations.

\subsubsection{Time Mean Flow}

Although the total energy has a bimodal distribution, the time averaged streamfunction and potential vorticity fields are still useful in describing the system. Averaged over the whole time series, the time mean streamfunction field (Figure 4-26a) is characterized by two antisymmetric recirculating gyres pressed against the eastward jet and the western boundary currents. The meridional and longitudinal sizes of the gyres are 200 and $1000 \mathrm{~km}$ respectively. East of the recirculations, the jet widens up to $400 \mathrm{~km}$.

The corresponding potential vorticity field (Figure 4-26b) shows two tongues of anomalously high and low potential vorticity advected by the western boundary current towards the middle of the domain from the north and south. The recirculation regions are marked by closed potential vorticity contours. The value of homogenized potential vorticity in the northern recirculation indicates that water is brought from the north, entrained into the jet and then mixed north of the jet. The opposite is true of the southern recirculation. The potential vorticity front associated with the eastward jet is sharp near the separation point and becomes wider east of $500 \mathrm{~km}$, which might be the sign of enhanced cross-front mixing in this area. 
(a)

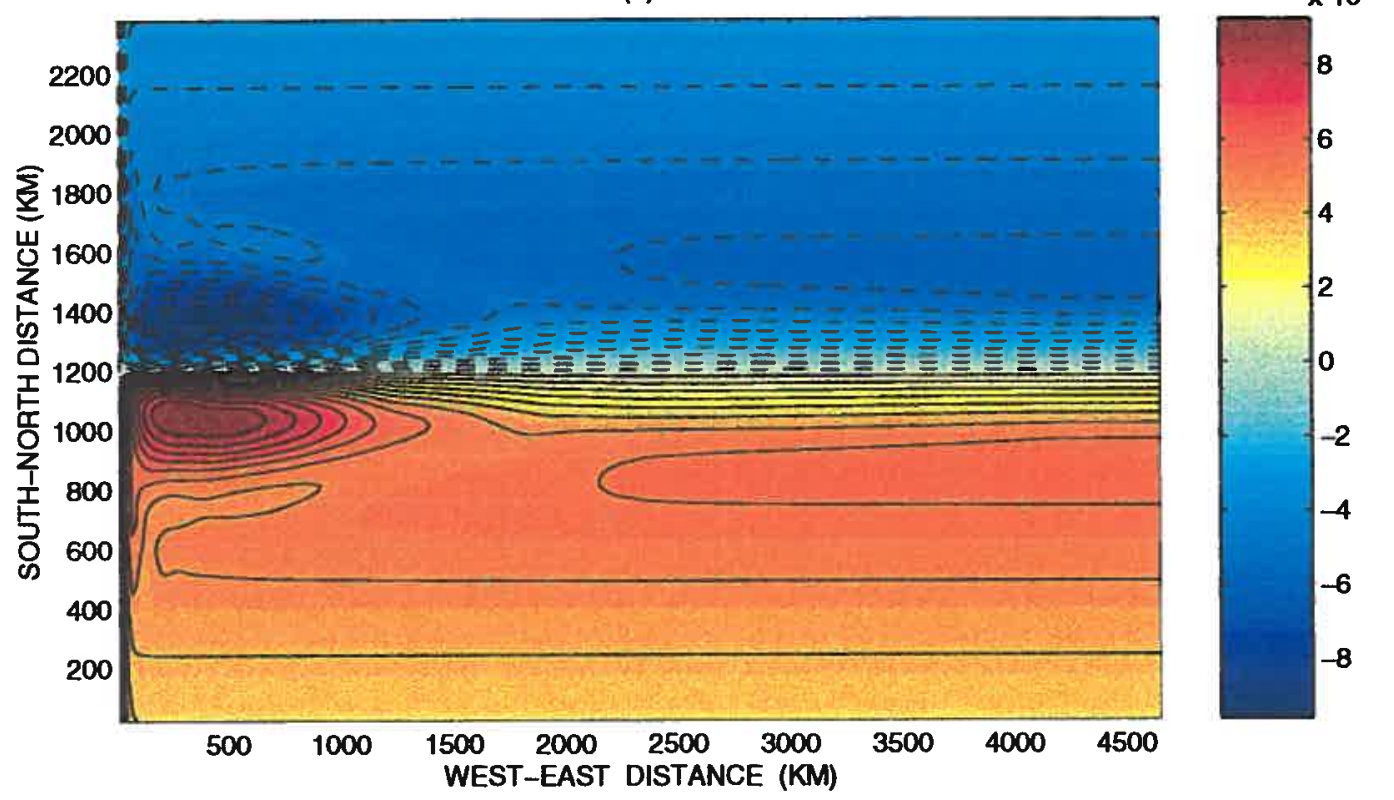

(b)

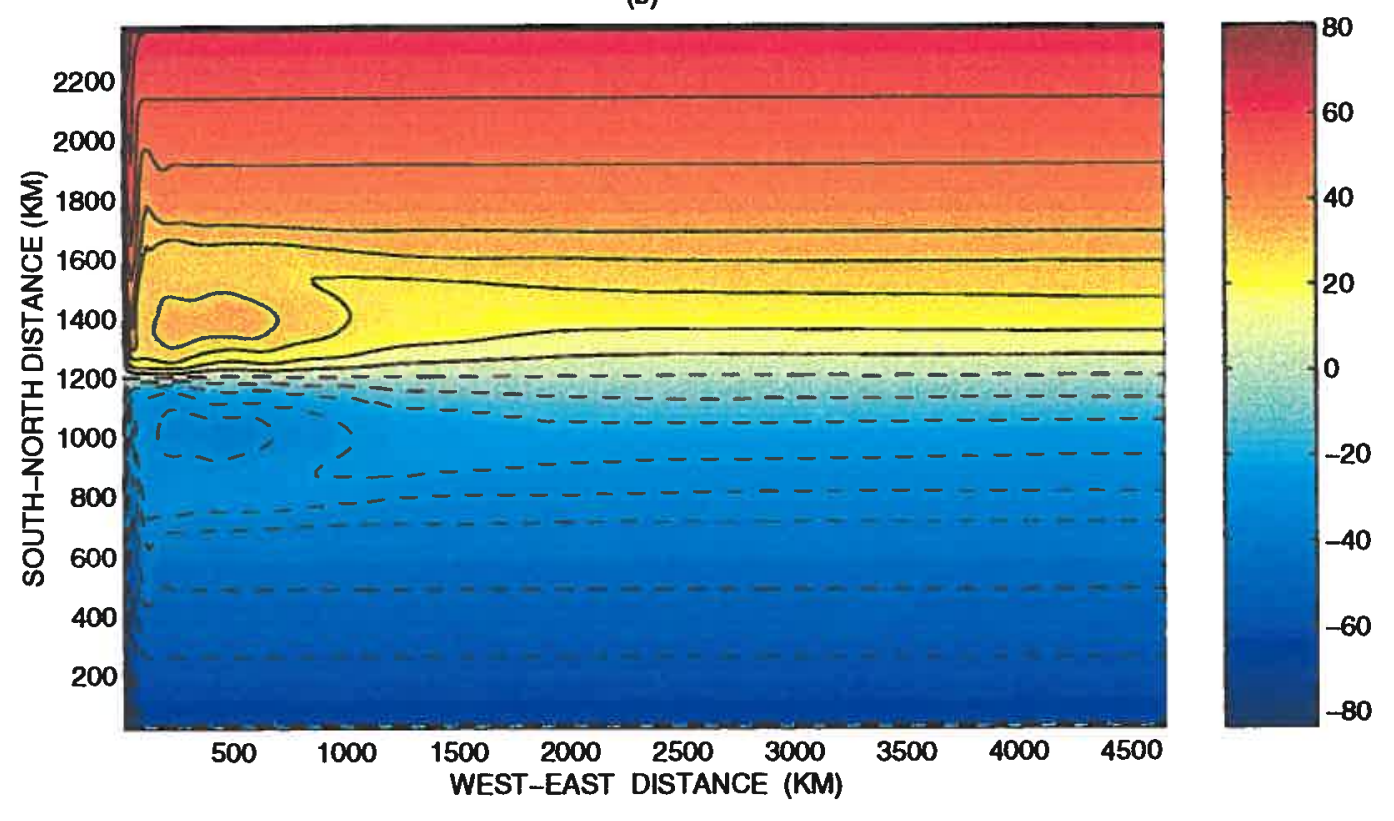

Figure 4-26: Contours of the time mean streamfunction field (a) and potential vorticity field (b). Dashed line denotes negative values, solid line denotes positive values. Parameters: $T_{\text {in }}=16 \mathrm{~Sv}, \mathcal{A}_{6}^{\text {dim }}=8 \cdot 10^{10} \mathrm{~m}^{4} \mathrm{sec}^{-1}$ and $\mathcal{A}_{2}^{\text {dim }}=3.0 \cdot 10^{-8} \mathrm{sec}^{-1}$. 
(a)

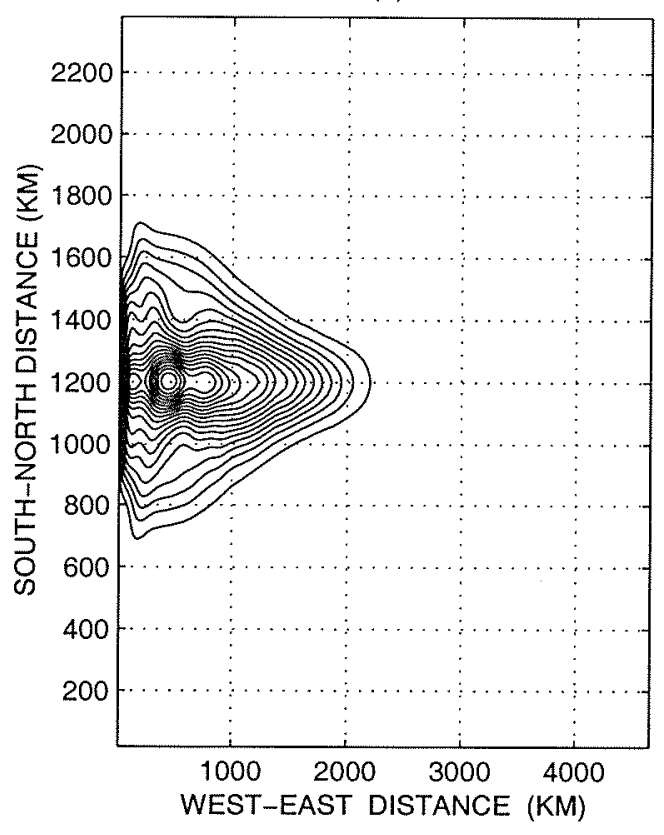

(c)

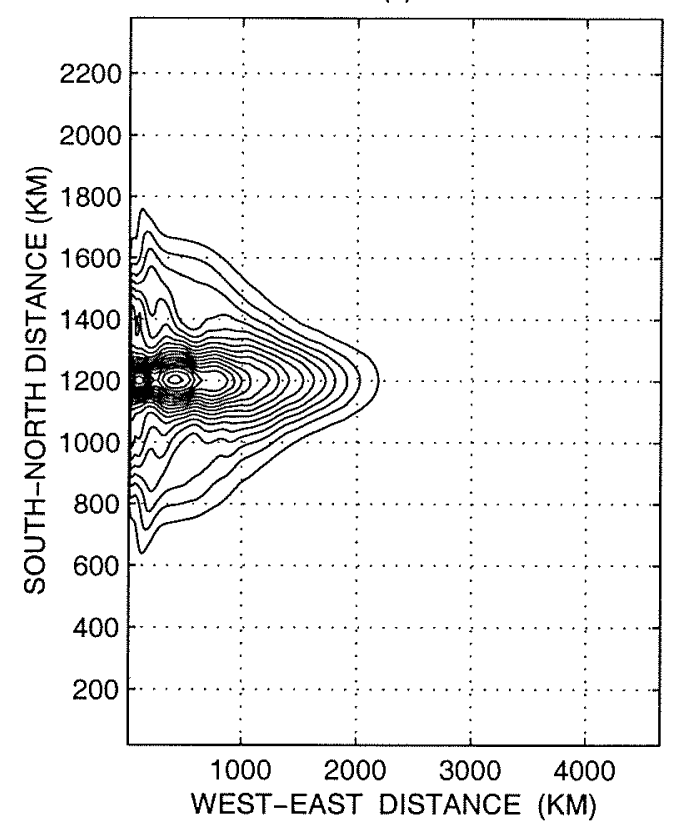

(b)

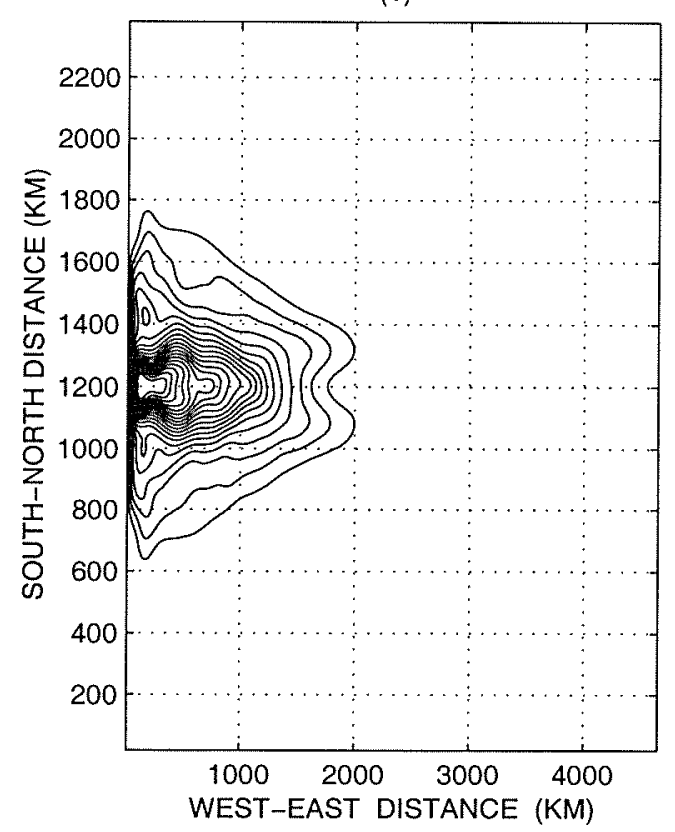

(d)

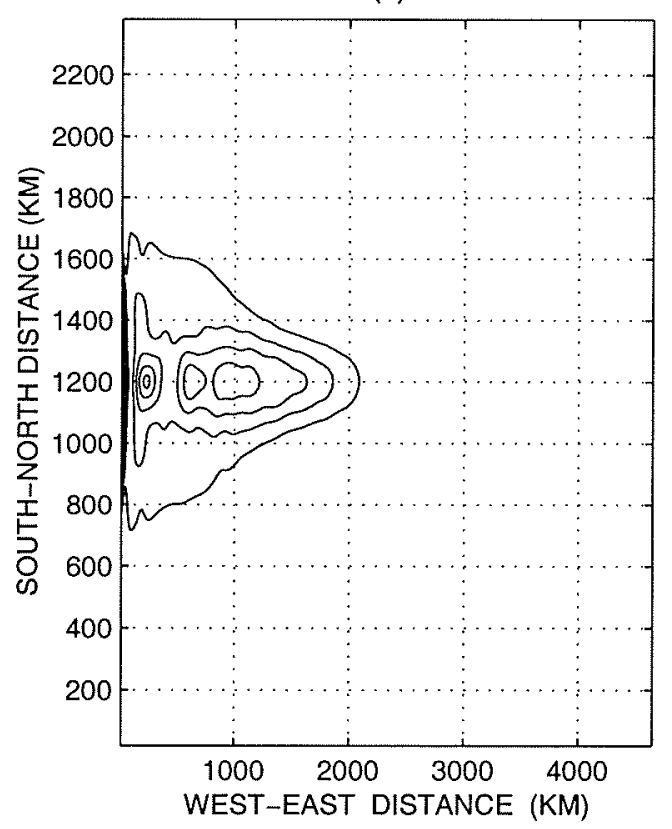

Figure 4-27: Time mean eddy fields of $\overline{\psi^{\prime}(x, y)^{2}}(\mathrm{a}), \overline{q^{\prime}(x, y)^{2}}(\mathrm{~b}), \overline{u^{\prime}(x, y)^{2}}(\mathrm{c}), \overline{v^{\prime}(x, y)^{2}}$ (d). Parameters: $T_{\text {in }}=16 \mathrm{~Sv}, \mathcal{A}_{6}^{\text {dim }}=8 \cdot 10^{10} \mathrm{~m}^{4} \mathrm{sec}^{-1}$ and $\mathcal{A}_{2}^{d i m}=3.0 \cdot 10^{-8} \mathrm{sec}^{-1}$. 


\subsubsection{Time Mean Eddy Variability}

The spatial structure of the time mean eddy variances (Figures 4-27a-d) indicates that the variability is concentrated in the area of the eastward jet and its recirculations and in the western boundary region close to the separation point. Within the eastward jet, the variability reaches its maximum around $800-1000 \mathrm{~km}$ and decays further eastward leveling to zero beyond $2000 \mathrm{~km}$. Another maximum is aligned with the western boundary current between 900 and $1500 \mathrm{~km}$. As will be discussed later, the conical shape of the variability region is associated with the barotropic instabilities in the form of waves, rings and meanders and their interactions with the western boundary current, changes in the spatial structure and the size of the recirculation gyres and meridional shifts in the position of the separation point of the eastward jet.

\subsubsection{Statistical Differences in the Eddy and Time Mean Flow Energy}

Figures $4-28 \mathrm{a}, \mathrm{b}$ present a segment of the total energy and the corresponding eddy energy time series. Visually, the low total energy periods are correlated with the periods of maximum eddy energy. To quantify it, we computed a two-dimensional histogram (Figure 4-29) showing the number of times the model had a particular total energy/eddy energy pair. When interpreting the results, one must remember that total energy is the sum of mean flow energy, eddy energy and temporary positive or negative correlations between the mean and fluctuating fields. The histogram shows two separate clusters of the eddy energy values. The first one is associated with the high total energy state, $\mathbf{H}$, and has the following mean and standard deviation: $\left(m_{H}, \sigma_{H}\right)=(0.0105,0.037)$. The second cluster corresponds to L-state and has $\left(m_{L}, \sigma_{L}\right)=(0.0163,0.041)$ as mean and standard deviation. The above analysis suggests that the high (low) total energy regimes are associated with the low (high) eddy energy. The small overlap between the two eddy states indicates that in addition to 
(a)

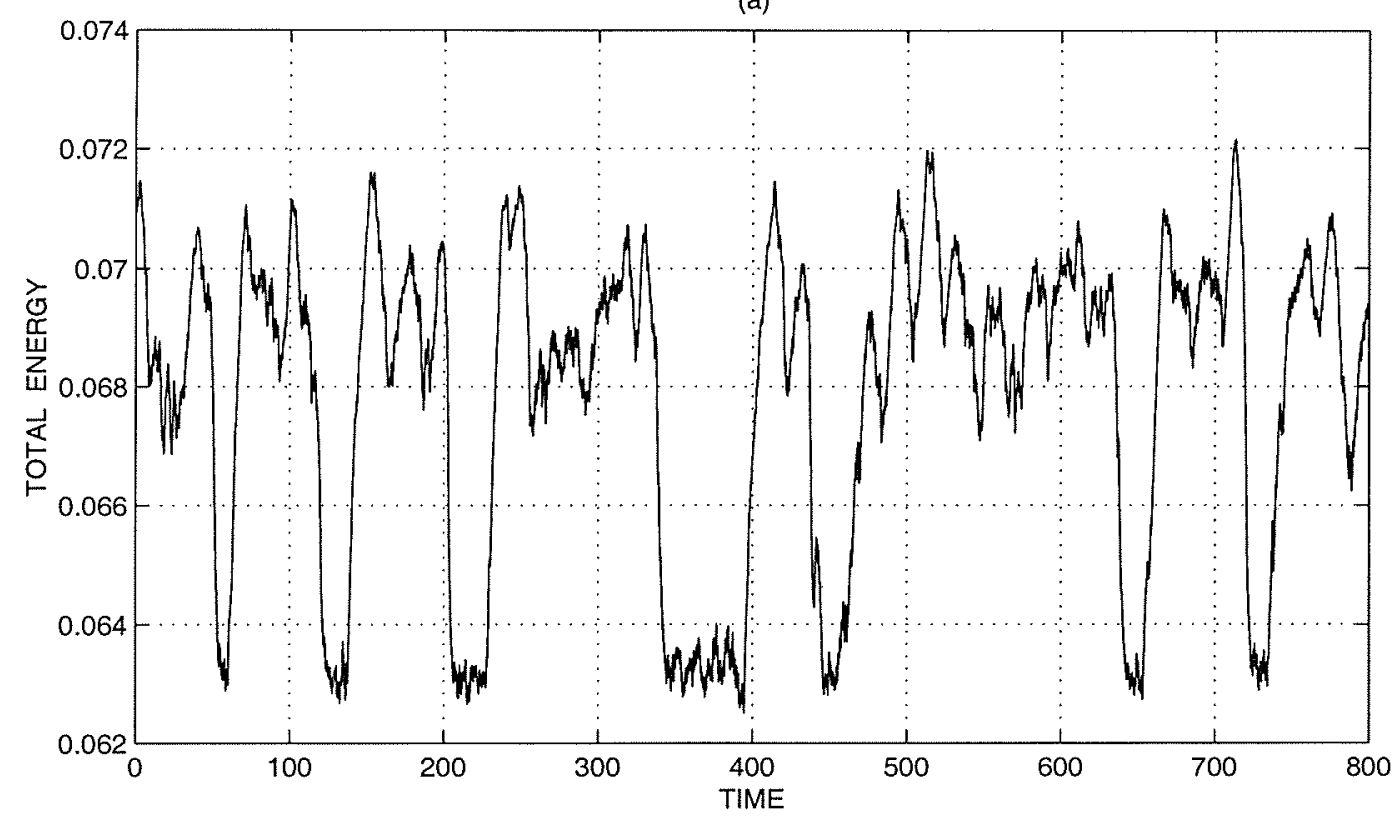

(b)

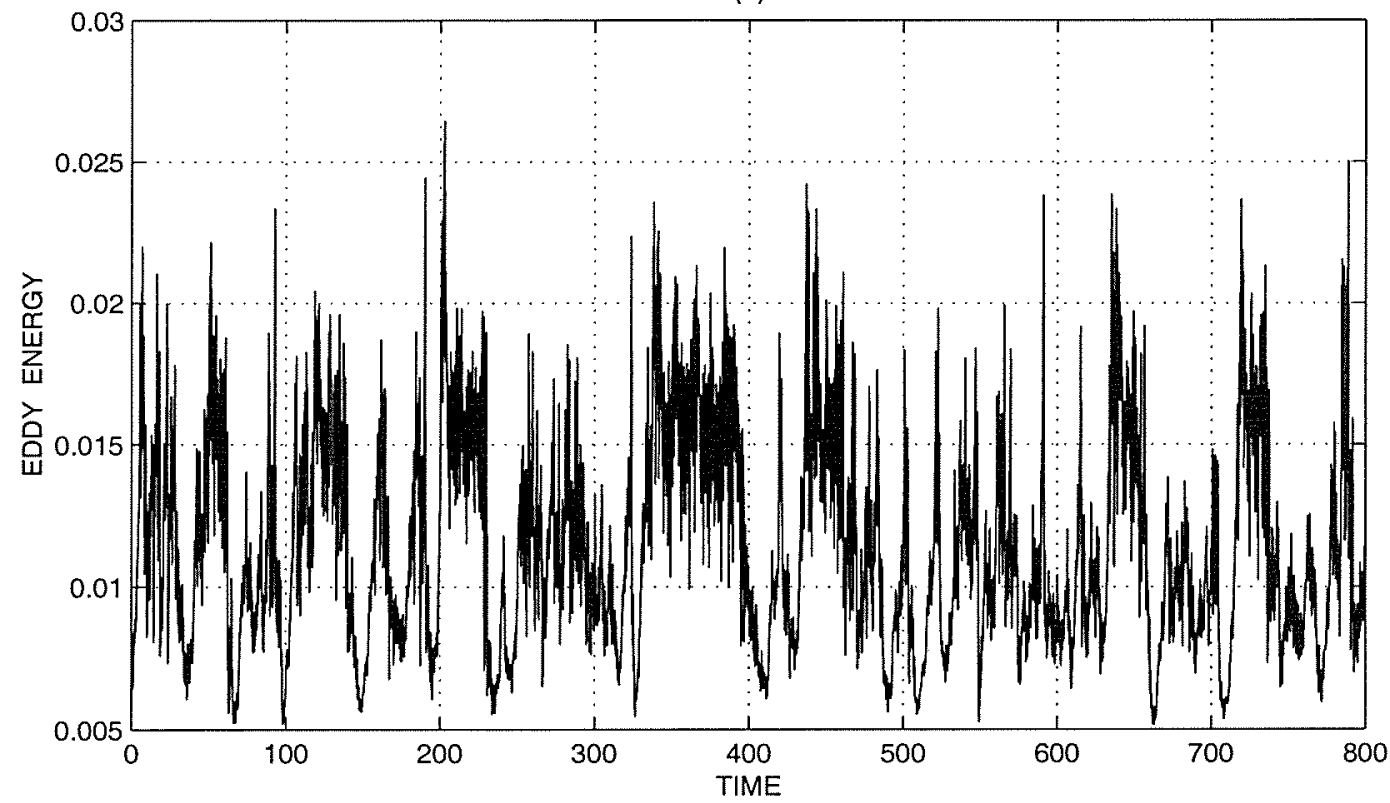

Figure 4-28: A segment of the time series of nondimensional total energy (a) and of corresponding nondimensional total eddy energy (b). Parameters: $T_{i n}=16 \mathrm{~Sv}$, $\mathcal{A}_{6}^{\text {dim }}=8 \cdot 10^{10} \mathrm{~m}^{4} \mathrm{sec}^{-1}$ and $\mathcal{A}_{2}^{\text {dim }}=3.0 \cdot 10^{-8} \mathrm{sec}^{-1}$. 


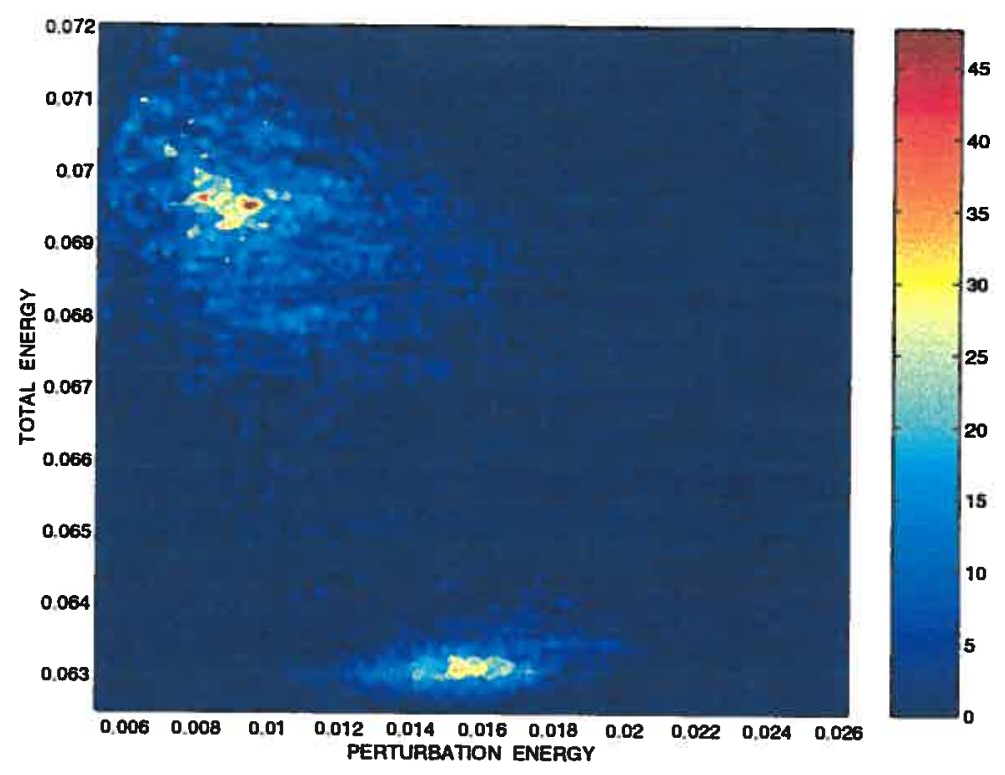

Figure 4-29: Two-dimensional histogram of a segment of the total energy and the total eddy energy from Figure 4-28. Parameters: $T_{i n}=16 \mathrm{~Sv}, \mathcal{A}_{6}^{\text {dim }}=8 \cdot 10^{10} \mathrm{~m}^{4}$ $\sec ^{-1}$ and $\mathcal{A}_{2}^{\text {dim }}=3.0 \cdot 10^{-8} \mathrm{sec}^{-1}$.

changes in the eddy intensity, there might be some structural differences in the eddy field.

\subsubsection{Enstrophy}

Figure 4-30a presents the histogram of total nondimensional enstrophy for the reference run. Clearly, similar to total energy, it has a bimodal distribution with wellseparated peaks associated with two dynamical regimes. Hence, in addition to total energy, total enstrophy could be another indicator of different dynamical regimes. The distribution of perturbation enstrophy (Figure 4-30b), although also bimodal, has a significant overlap.

Total enstrophy length scale can be defined as:

$$
L_{\text {enst }}=L \sqrt{\frac{E}{\epsilon}}
$$

where $L=2400 \mathrm{~km}$ is model's horizontal length scale, $E$ is domain-integrated total nondimensional energy and $\epsilon=\int_{y_{s}}^{y_{n}} \int_{x_{w}}^{x_{e}} \frac{q^{2}}{2} d y d x$ is total nondimensional enstrophy. 

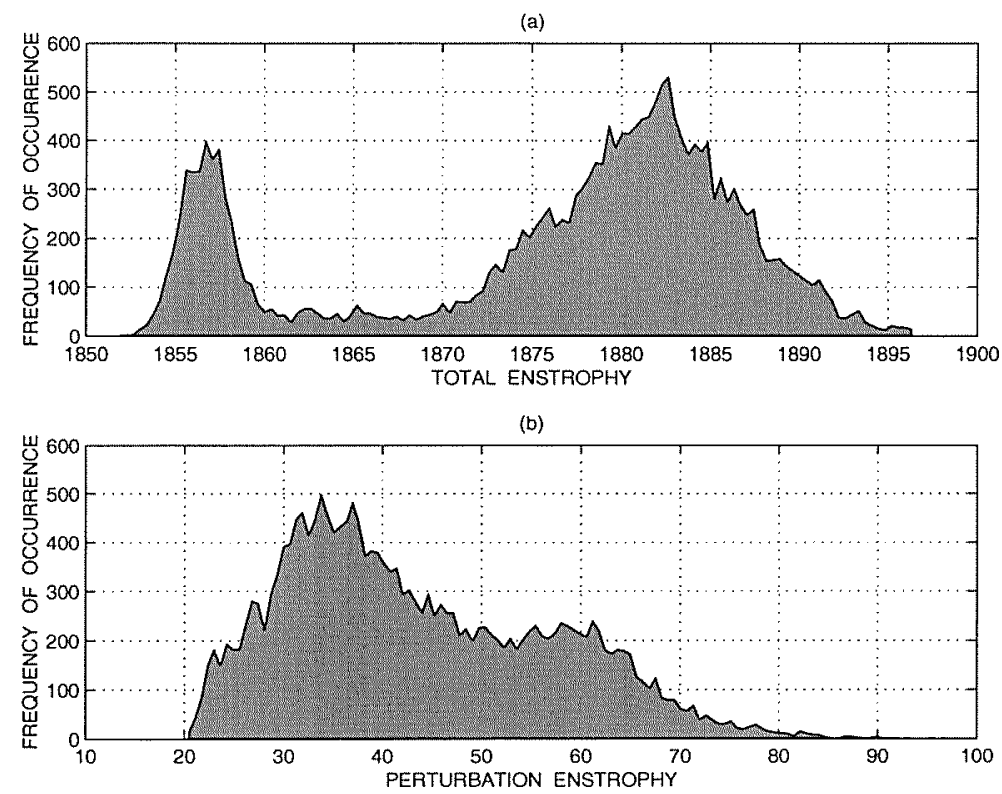

Figure 4-30: (a): Histogram of total nondimensional enstrophy. (b) Histogram of perturbation nondimensional enstrophy. Parameters: $T_{i n}=16 \mathrm{~Sv}, \mathcal{A}_{6}^{\text {dim }}=8 \cdot 10^{10} \mathrm{~m}^{4}$ $\sec ^{-1}$ and $\mathcal{A}_{2}^{\text {dim }}=3.0 \cdot 10^{-8} \mathrm{sec}^{-1}$.

Similarly, eddy enstrophy length scale, $L_{\text {enst }}^{\prime}$, is defined in (4.11), where $E$ is now the domain-integrated perturbation energy and $\epsilon=\int_{y_{s}}^{y_{n}} \int_{x_{w}}^{x_{e}} \frac{q^{\prime 2}}{2} d y d x$ is eddy enstrophy.

Figure 4-31 shows two-dimensional histograms of total energy vs $L_{\text {enst }}$ and of eddy energy vs $L_{\text {enst }}^{\prime}$. Clearly, there is no significant change in the enstrophy length scale between the high and low energy regimes. Therefore, regime variability is not connected to scale variability.

\subsubsection{Mean Fields Associated with Different Energy States}

The averaging of the fields within several energy bands shows qualitative differences in the structure of the flow and helps to understand the nature of the multiple dynamical states of the model. Figures 4-32 and 4-33 present ensemble averages of the streamfunction and potential vorticity over four energy bands: low energy: $\mathrm{L}=[0.062$ 0.064], transition and a low range of the high energy state: $\mathrm{HL}=[0.064,0.0685]$, a 
(a)
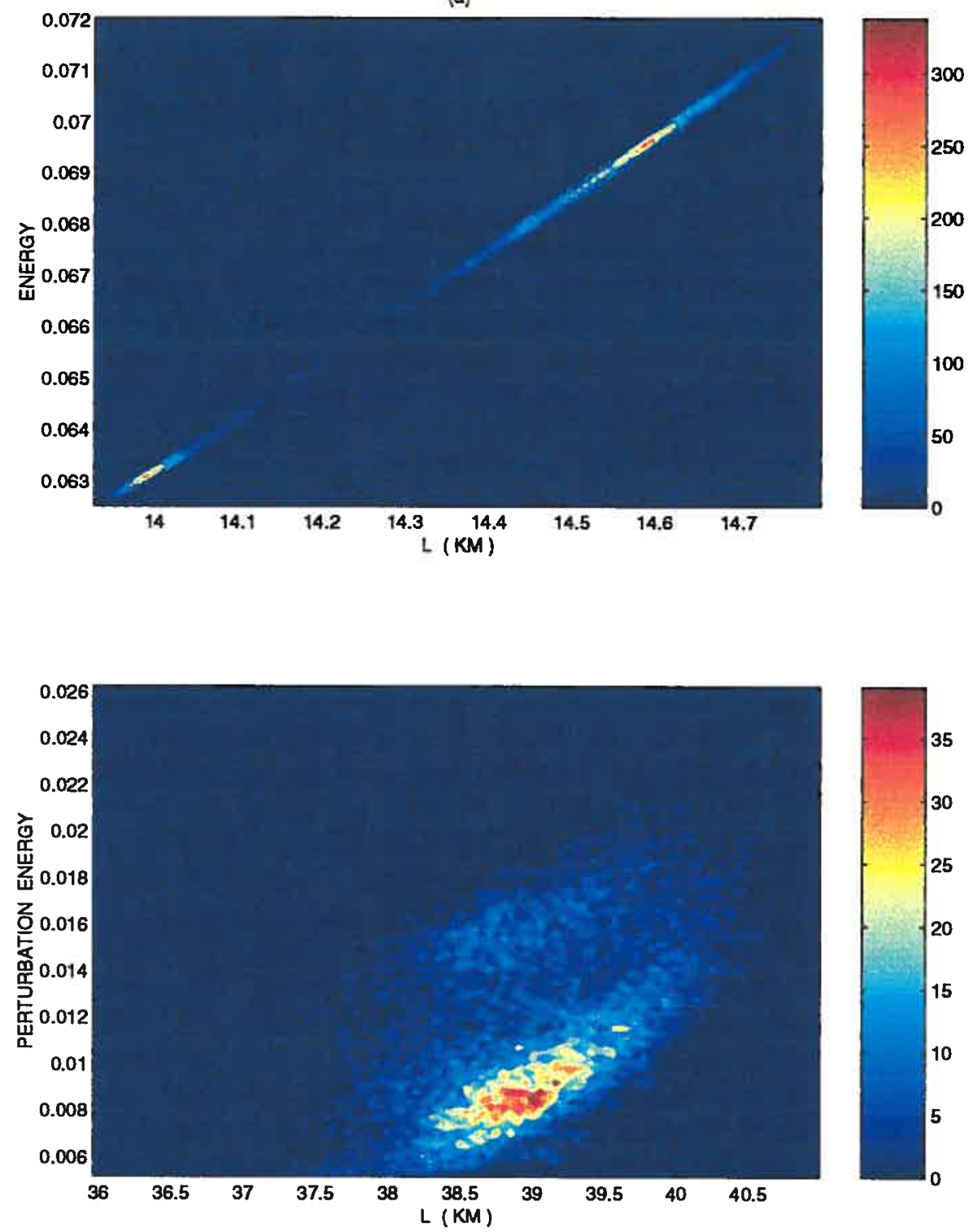

Figure 4-31: (a): Two-dimensional histogram of nondimensional total energy, $E$, vs dimensional total enstrophy scale, $L_{\text {enst }}=L \sqrt{\frac{E}{0.5 q^{2}}}$, where $q$ is potential vorticity. (b): Two-dimensional histogram of nondimensional perturbation energy, $E$, vs dimensional perturbation enstrophy scale, $L_{\text {enst }}=L \sqrt{\frac{E}{0.5 q^{2}}}$, where $q^{\prime}$ is perturbation potential vorticity. Parameters: $T_{\text {in }}=16 \mathrm{~Sv}, \mathcal{A}_{6}^{\text {dim }}=8 \cdot 10^{10} \mathrm{~m}^{4} \mathrm{sec}^{-1}$ and $\mathcal{A}_{2}^{\text {dim }}=3.0 \cdot 10^{-8} \mathrm{sec}^{-1}, L=2400 \mathrm{~km}$. 
(a)

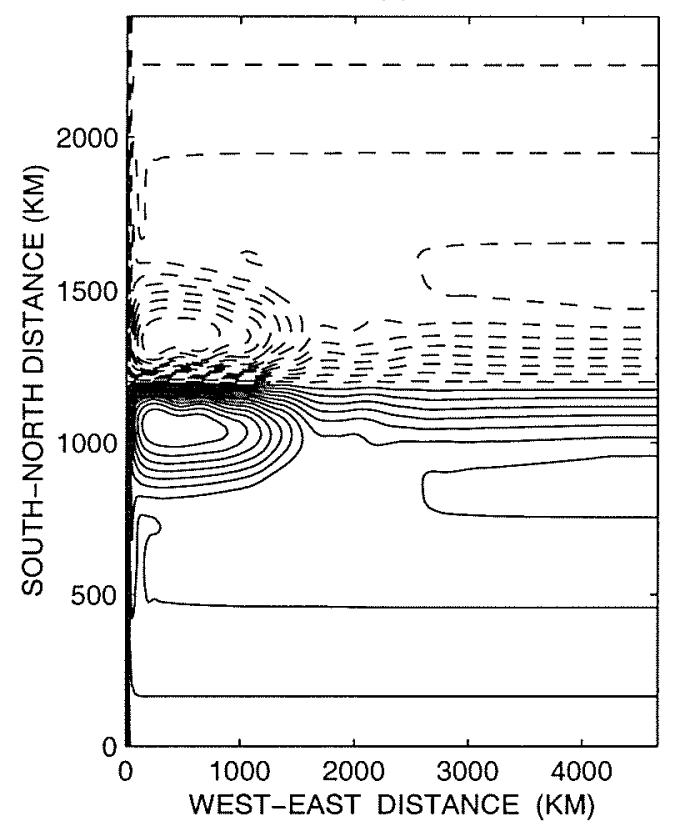

(c)

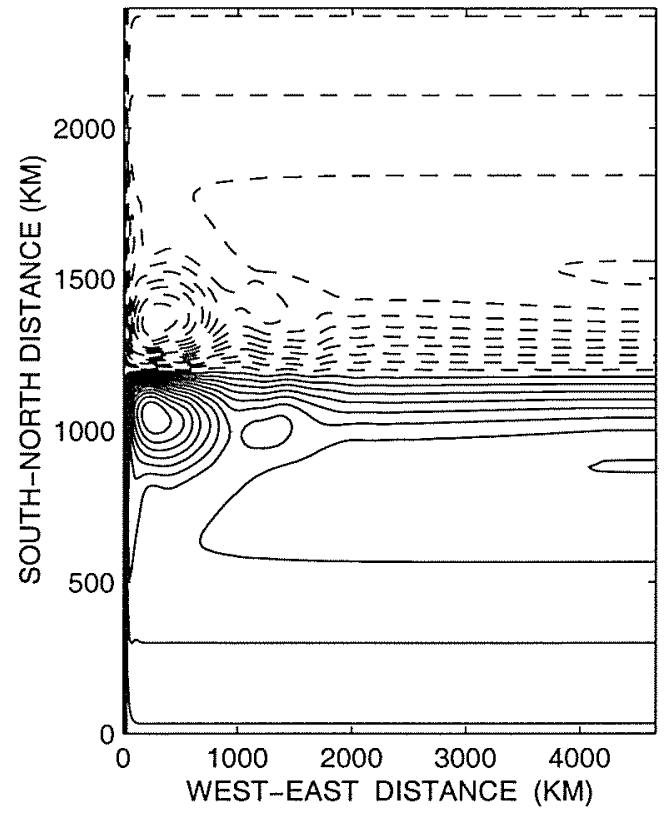

(b)

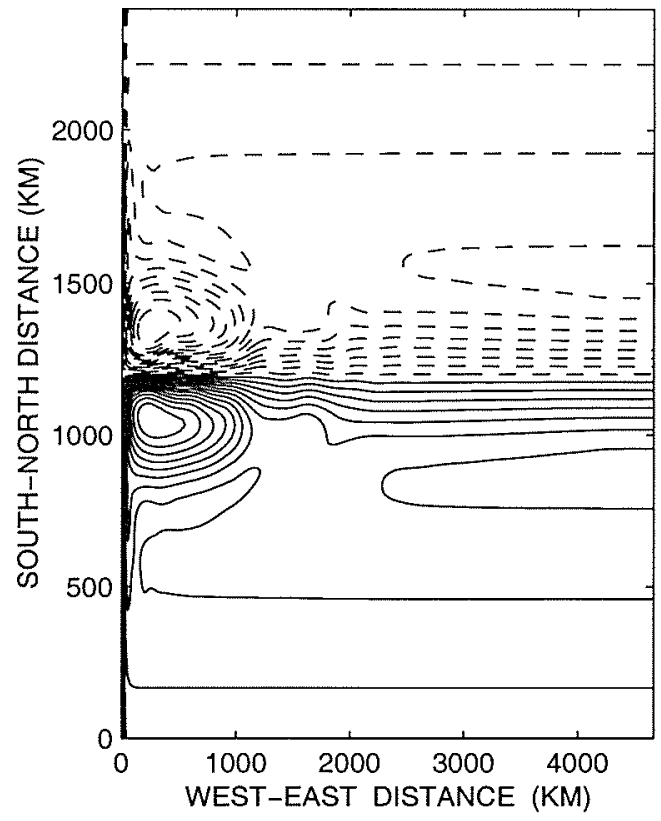

(d)

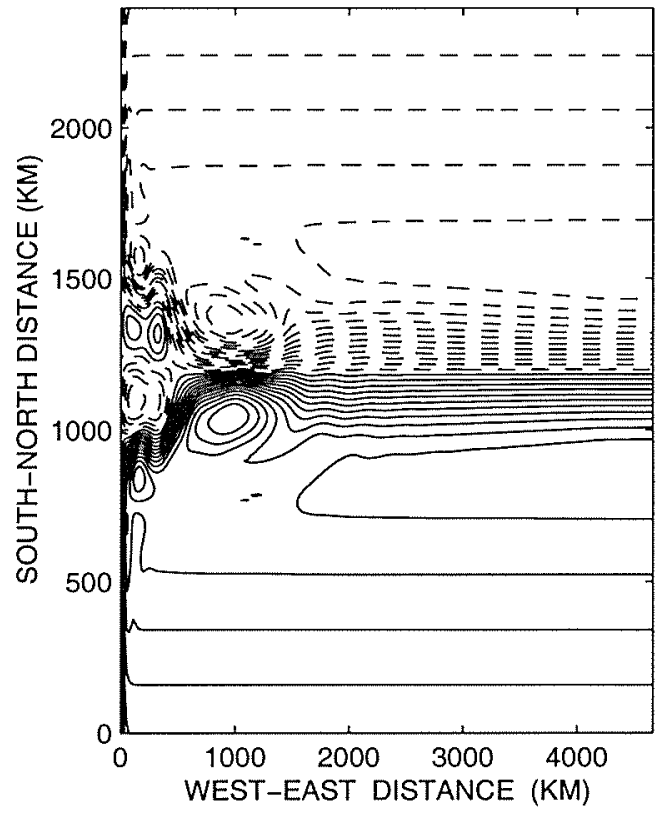

Figure 4-32: Ensemble average of the streamfunction fields with the total energy levels for HH-state $(T E>0.0705)$ (a), HM-state $(0.0685<T E<0.0705)$ (b), HL-state $(0.064<T E<0.0685)(\mathrm{c})$ and L-state $(T E<0.064)$ (d). Parameters: $T_{\text {in }}=16 \mathrm{~Sv}$, $\mathcal{A}_{6}^{\text {dim }}=8 \cdot 10^{10} \mathrm{~m}^{4} \mathrm{sec}^{-1}$ and $\mathcal{A}_{2}^{\text {dim }}=3.0 \cdot 10^{-8} \mathrm{sec}^{-1}$. 
(a)

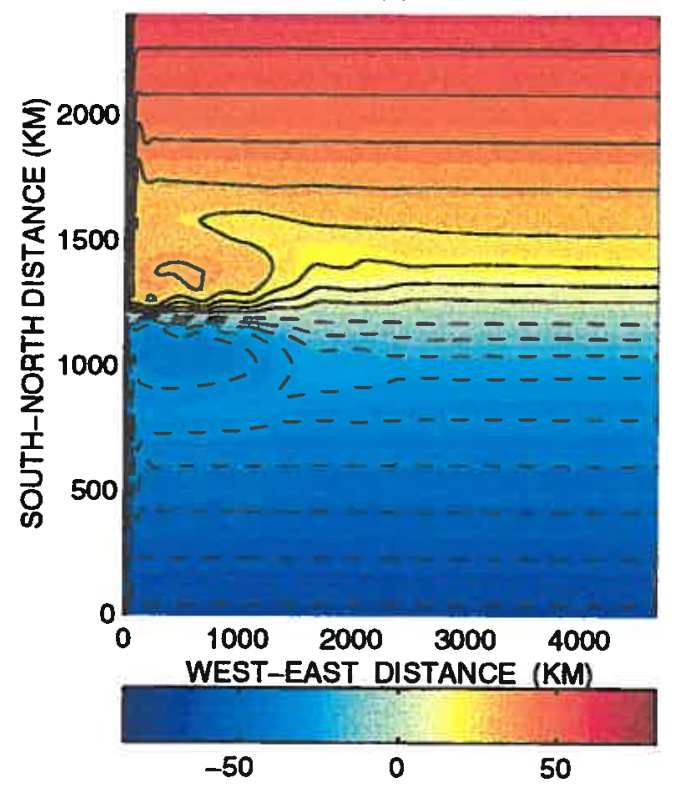

(c)

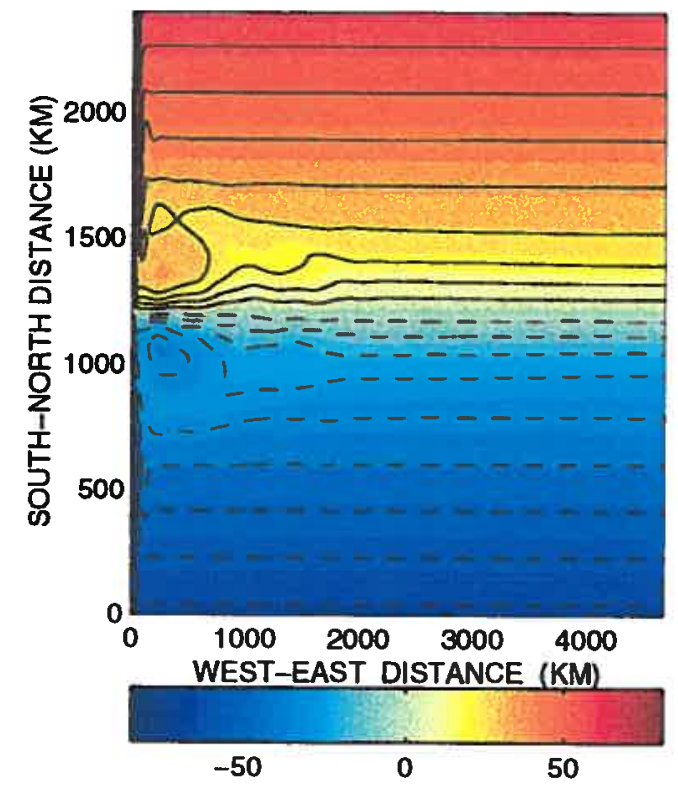

(b)

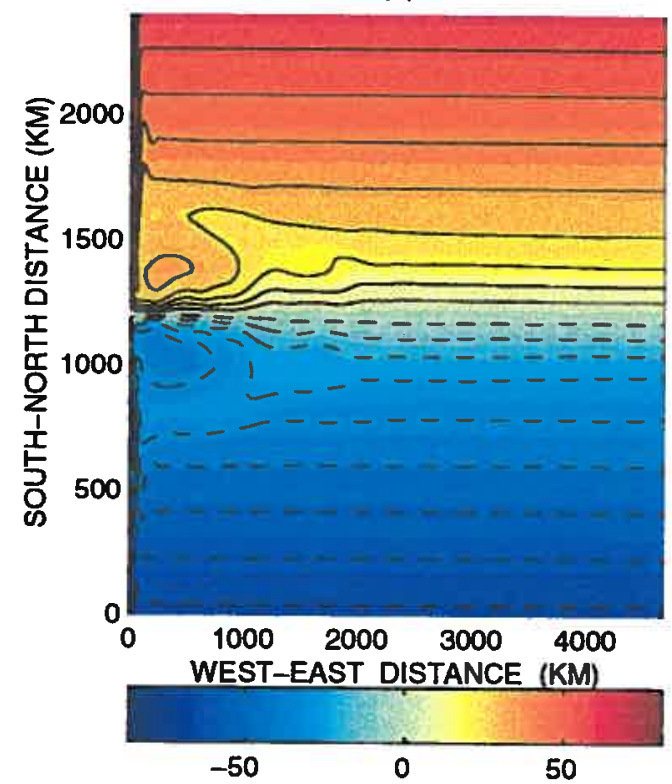

(d)

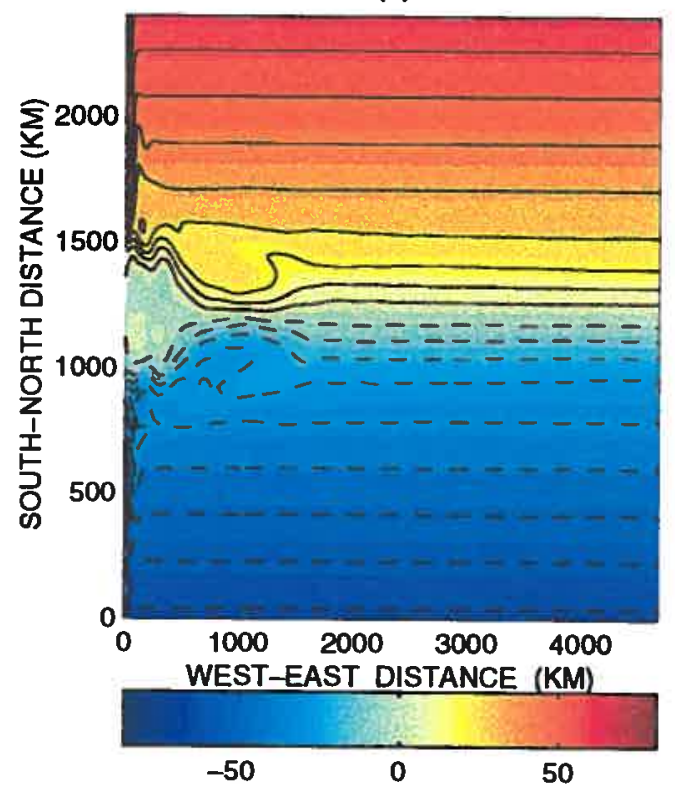

Figure 4-33: Ensemble average of potential vorticity fields with the total energy levels for HH-state $(T E>0.0705)$ (a), HM-state $(0.0685<T E<0.0705)$ (b), HL-state $(0.064<T E<0.0685)$ (c) and L-state $(T E<0.064)(\mathrm{d})$. Parameters: $T_{\text {in }}=16 \mathrm{~Sv}$, $\mathcal{A}_{6}^{d i m}=8 \cdot 10^{10} \mathrm{~m}^{4} \mathrm{sec}^{-1}$ and $\mathcal{A}_{2}^{d i m}=3.0 \cdot 10^{-8} \mathrm{sec}^{-1}$. 
medium range of the high energy state: $H M=[0.0685,0.0705]$ and a high range of the high energy state: $\mathbf{H H}=[0.0705,0.072]$.

The structures of the streamfunction fields are similar for the last three energy ranges: all of them are characterized by the presence of symmetric recirculations. However, the longitudinal size of the gyres decreases from $1500 \mathrm{~km}$ for HH-state to $800 \mathrm{~km}$ for HL-state. In HM- and HL- states, the width of the eastward jet increases from $200 \mathrm{~km}$ just east of the recirculations to $400 \mathrm{~km}$ east of $2000 \mathrm{~km}$. The sudden change in the jet's width is associated with the presence of larger meanders and the formation of rings east of the recirculation gyres. The corresponding potential vorticity fields differ not only by the shape of the recirculation regions, but also by the values of the mixed $q: \mathbf{H H}$ has the highest values of anomalous potential vorticity. Another obvious difference is the detachment of the mixed $q$-region from higher and lower latitudes in HL-state, which allows for larger excursion of potential vorticity contours indicating a more unstable flow structure.

In the L-energy range, the time mean field is a combination of equally possible antisymmetric solutions. One is characterized by the overshooting southward-flowing western boundary current separating from the wall approximately $300 \mathrm{~km}$ south of the middle of the domain. The other one has a northward-flowing western boundary current separating $300 \mathrm{~km}$ north of the middle of the domain. Such solutions explain the presence in the time mean of an anticyclonic/cyclonic vortex north/south of the middle of the domain. In the time mean, the recirculations appear to be pushed further eastward, and their zonal extent is only $500 \mathrm{~km}$. They exist mostly in the time mean sense; the instantaneous fields have individual vortices confined between large amplitude, westward propagating meanders. The corresponding time mean potential vorticity field appears to have a $500 \mathrm{~km}$ by $500 \mathrm{~km}$ region of "homogenized" potential vorticity in the western part of the domain. This is misleading, since the time mean field is constructed by averaging of two opposite sign potential vorticity fields due to overshooting events. Nevertheless, this is a region of vigorous mixing 
across the front, since large amplitude, westward propagating meanders interacting with the western wall form strong rings, that propagate away from the middle of the domain.

\subsubsection{Typical Energy Cycle}

\section{Eddy Energy - Total Energy Partition}

To understand better the nature of multiple dynamical regimes, we choose a typical cycle (Figure 4-34a) when the total energy rapidly decays (HL-transition) and, after fluctuating around minimum values for 10-50 years, quickly builds up again reaching its maximum (LH-transition). Typical transitions take between 5 and 10 years and are associated with significant changes in the spatial structure of the mean and eddy fields. Such changes are dynamically asymmetric for HL- and LH-transitions. The ratio of eddy to total energy (Figure 4-34b) indicates that in the former case, the eddy field part increases from $10 \%$ to $30 \%$. During the $\mathbf{L H}$-transition, the eddy energy level reaches its minimum of $7 \%$ and averages at $15 \%$ in $\mathbf{H}$-state. The amplitude of the fluctuations in the eddy-mean flow partition is the highest during $\mathbf{L}$-state.

\section{Flow Evolution During a Typical Energy Cycle}

In this section, we describe the evolution of the streamfunction and potential vorticity fields during a typical energy cycle depicted in Figure 4-34a. At $t=1.3$ years (denoted by number 1 in Figure 4-34a), when the total energy is in $\mathrm{H}$-state, the flow (Figures 4-35a,b) has well-developed recirculations with a longitudinal extent of $1500 \mathrm{~km}$. Small fluctuations in the position of the separation point are due to weak eastward propagating meanders. East of the recirculation region, where the jet is wider and therefore more stable, meanders are characterized by westward propagation and larger amplitudes. The collision of eastward and westward propagating meanders leads to formation of rings in the region east of the recirculations. The 
(a)

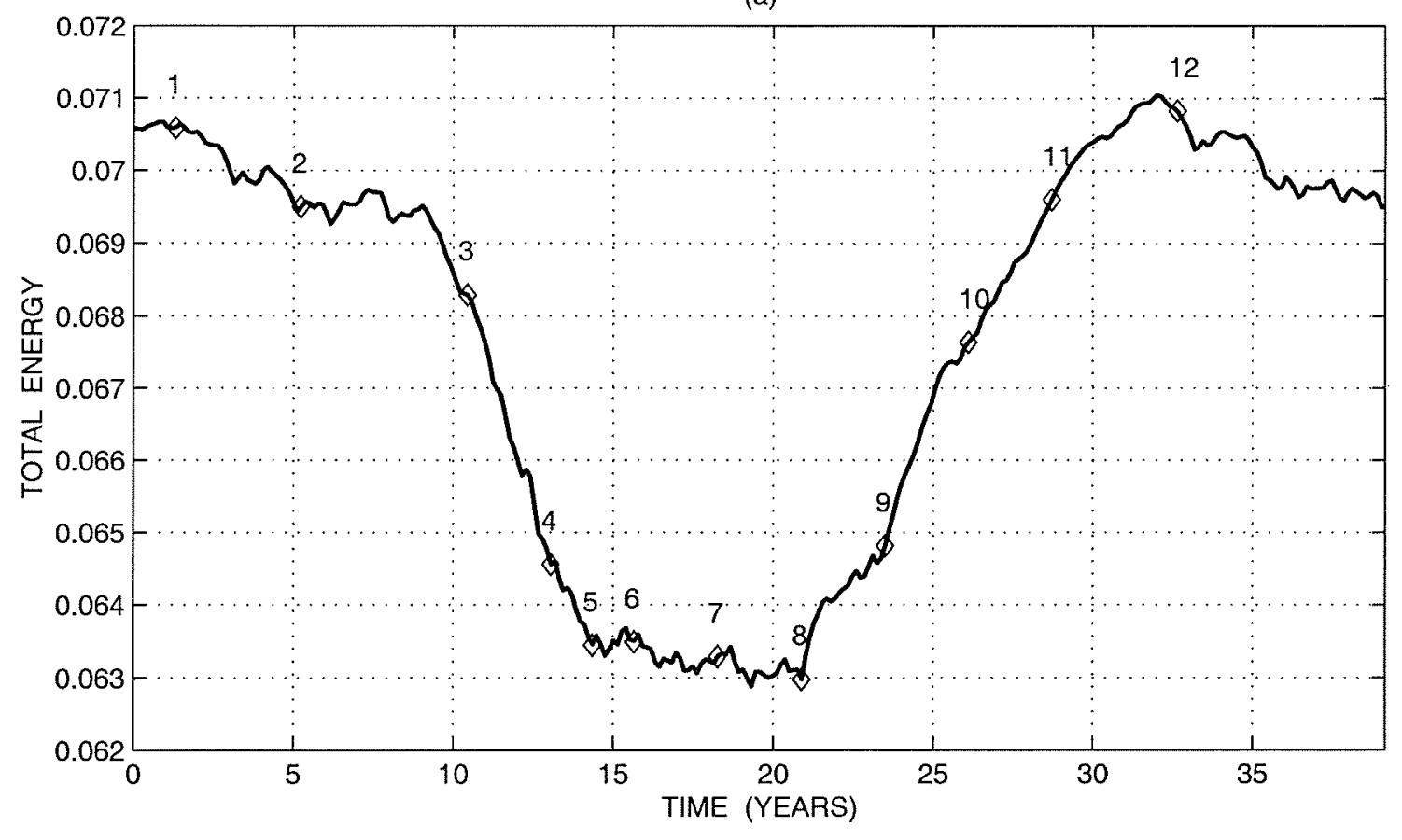

(b)

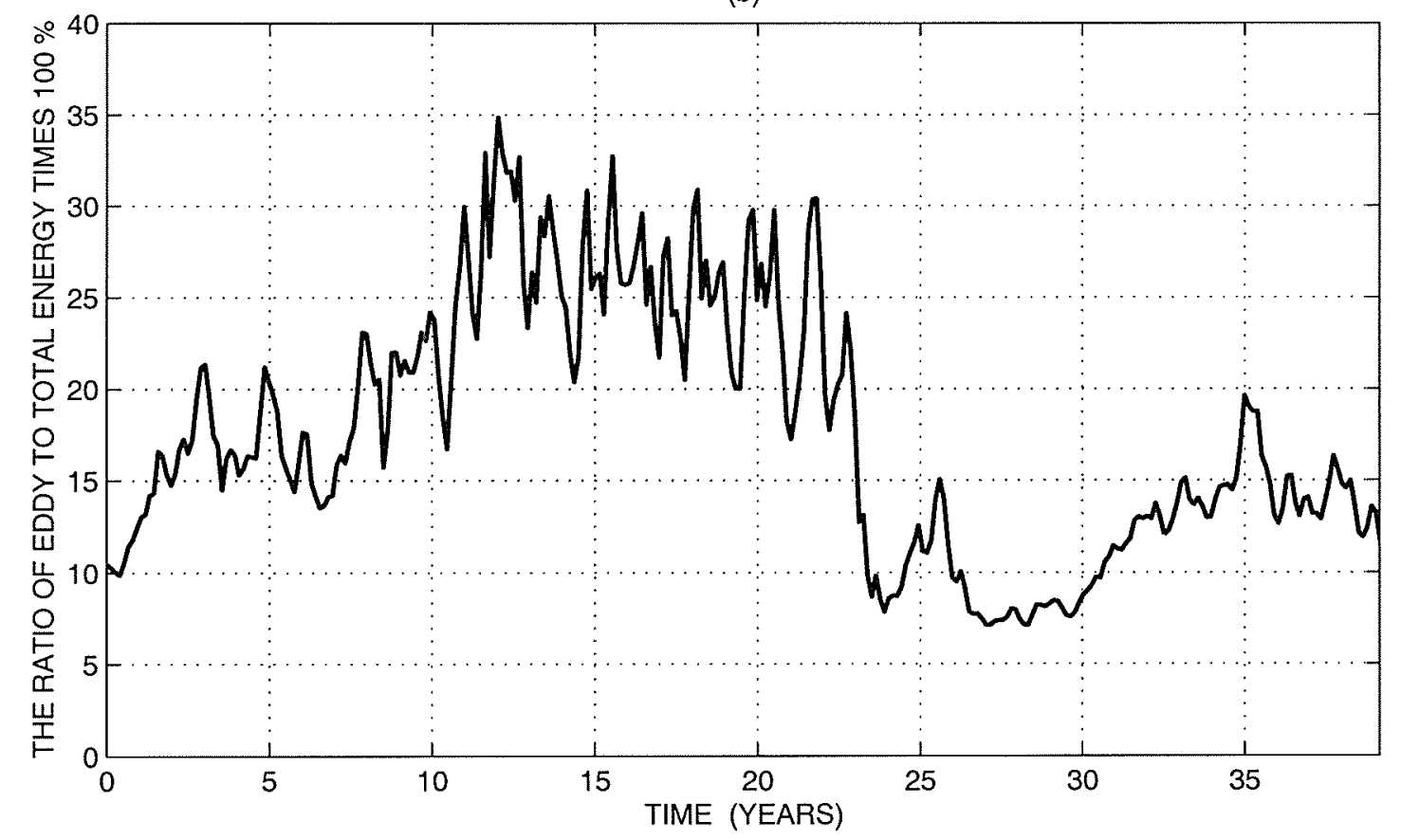

Figure 4-34: (a): Segment of the total energy time series corresponding to a typical low frequency cycle. (b): The corresponding ratio of eddy to total energy times $100 \%$. Parameters: $T_{\text {in }}=16 \mathrm{~Sv}, \mathcal{A}_{6}=8 \cdot 10^{10} \mathrm{~m}^{4} \mathrm{sec}^{-1}$ and $\mathcal{A}_{2}=3.0 \cdot 10^{-8} \mathrm{sec}^{-1}$. 
meander amplitudes define the strength and the size of created vortices and thus the degree of potential vorticity mixing across the front. Once the ring detaches from the front, it travels westward in the recirculating gyres mixing the opposite sign vorticity into the surroundings and homogenizing the surrounding gradient. Upon reaching the western wall, at this stage of the flow development, rings are not strong enough to overcome the advective influence of the western boundary current. Thus, they join the eastward jet and further perturb it.

At $t=5.2$ years (denoted by number 2 in Figure $4-34 \mathrm{a}$ ), the total energy level is still high, and the eddy energy partition increases from $10 \%$ to $20 \%$. The growth of jet instabilities and subsequent ring formation lead to a decrease to $800 \mathrm{~km}$ in the longitudinal extent of the recirculations (Figures 4-35c,d). Although the jet front is still symmetric in the recirculation region, the gyres lose their symmetry. One of them starts wrapping around another one, thus giving birth to stronger and larger rings and therefore providing enhanced potential vorticity mixing across the front. Rings in turn tend to further decrease the size and the strength of the recirculations.

Time $t=10.4$ years (denoted by number 3 in Figure 4-34a) marks the beginning of a sharp decline in the total energy level and corresponds to a further increase in the eddy energy partition. Although the jet still separates in the middle of the domain, its front exhibits strong northward and southward oscillations east of 500 $\mathrm{km}$. Figures 4-35e,f show one of the states, with a smaller northern recirculation and a northward front excursion. The latter results in a production of stronger and larger rings on the south and weaker and smaller rings on the north. Therefore, upon reaching the western wall, the rings have different trajectories; strong cyclonic rings are propelled southward, thereby decreasing the inflow from the south, whereas weak anticyclonic rings join the eastward jet without affecting the inflow from the north to any great extent. These processes create conditions favorable for the southward shift in the position of the separation point. The opposite is true when the front has a southward excursion. 
Figure 4-36 presents instantaneous streamfunction and potential vorticity fields at times $t=13,14.3$ and 15.6 years (denoted by numbers 4, 5 and 6 in Figure 4-34a) which correspond to a $30 \%$ maxima in the eddy energy partition. A sharp decay in the total energy level is due to the potential energy decline associated with an almost complete disappearance of the recirculations. Instead of the well-developed gyres, there are individual vortices corresponding to meander peaks and troughs east of 500 $\mathrm{km}$. Such structures provide efficient mixing on the flanks of an eastward jet. The separation point shifts $300 \mathrm{~km}$ south and north of the middle of the domain and, along with large amplitude meanders provides stronger fluctuations in the domainintegrated potential vorticity field.

During this stage of the flow development, there is no ring formation in the interior of the domain. Instead, strong rings are constantly formed on the western boundary in the following manner. Large amplitude meanders propagate westward similar to long Rossby waves. If a negative potential vorticity anomaly associated with a meander peak reaches the western wall, it starts moving northward due to the influence of an image anomaly arising from a no-normal-flow boundary condition. The separation point is therefore shifting northward. Meanwhile, an upstream positive potential vorticity anomaly corresponding to a meander trough continues to propagate westward. Upon hitting the western wall, it starts moving southward. Two anomalies propelling in the opposite directions cause the disruption in the potential vorticity front. As a result, a strong, northward propagating anticyclonic ring is formed from the negative potential vorticity anomaly, and the separation point jumps southward.

The process is repeated for the positive potential vorticity anomaly, which leads to further southward excursion of the separation point and to the formation of a strong, southward propagating cyclonic ring. The dynamics of such strong boundaryformed rings tends to restore the symmetry of the inflow conditions. However, the interaction of the large amplitude, westward propagating meanders leads to the persistence of a low energy state. 
The model stays in this dynamical regime until the instabilities start decaying, perhaps due to frictional effects, which results in the decrease of meander amplitudes and in the amplitude of the separation point excursions. The strength of the boundary formed rings also decreases, which helps to restore the inflow symmetry. At times $t=18.2$ and 20.8 years (Figures 4-37a-d), which corresponds to the middle and to the end of state $\mathbf{L}$ (denoted by numbers 7 and 8 in Figure 4-34a), the shifts of the separation point become so small that weak recirculations develop near the western boundary. At this point, rings form just east of the recirculations, and their detachment tends to flatten the front.

The transition to the high energy state is illustrated by Figures 4-37e-f and 438a-d, which are snapshots at $t=21,23.5,26.1$ and 28.7 years (denoted by numbers 9-12 in Figure 4-34a). The eddy energy partition drops below $10 \%$ during this period. The size of the recirculations increases from 500 to almost $2000 \mathrm{~km}$, and the ring formation process slows down and, at some point, halts. Thus, the model reaches the dynamical state associated with large recirculation gyres and very weak eddy activity. The spatial structure resembles the antisymmetric steady solution described in section 4.2.1.

However, the energy does not stay in its maxima for a long time. Instead, the model trajectory reaches the vicinity of an antisymmetric steady solution and then quickly escapes back to the regime where nonlinear eddy-eddy interactions are important. This might happen because the model has no mechanism that would restore the symmetry of the inflow conditions near the separation point and therefore guide the model trajectory towards the fixed point. In the wind-driven models with nearly antisymmetric wind-stress curl, the correlation between the wind-stress curl and the antisymmetric steady solution might be important in helping the model trajectory to reach a closer neighborhood of the fixed point. In agreement with the linear stability analysis of Primeau [29], it takes more than 20 years for the instabilities to grow and to move the wind-driven model from a high to a medium energy state. 
(a)

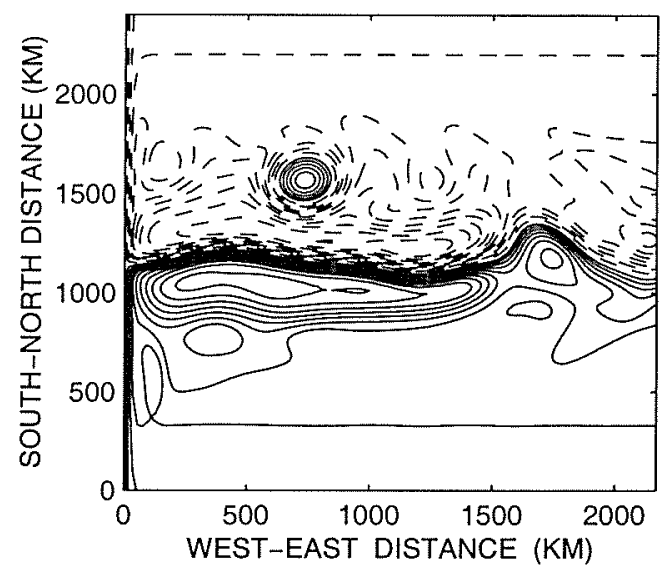

(c)

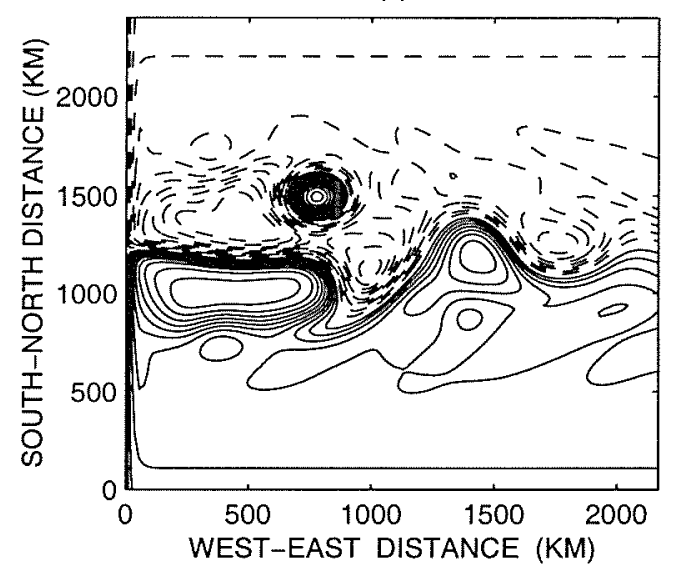

(e)

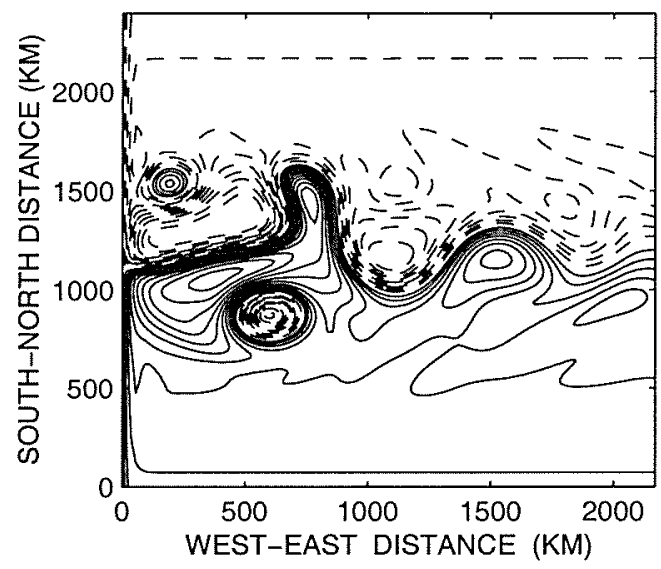

(b)

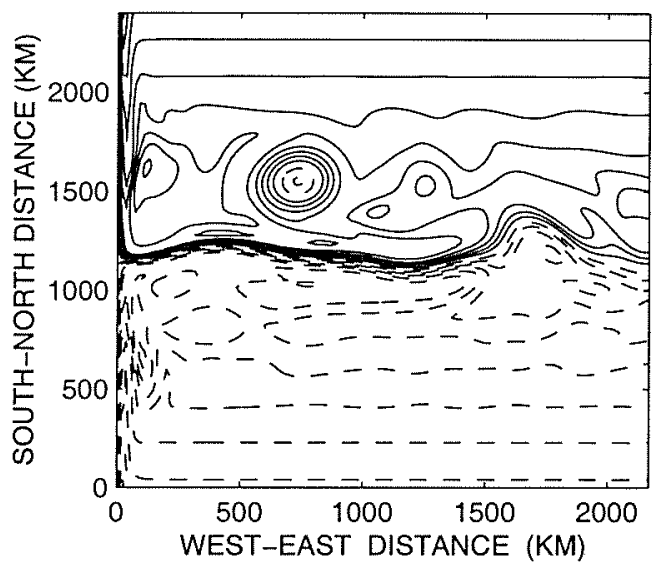

(d)

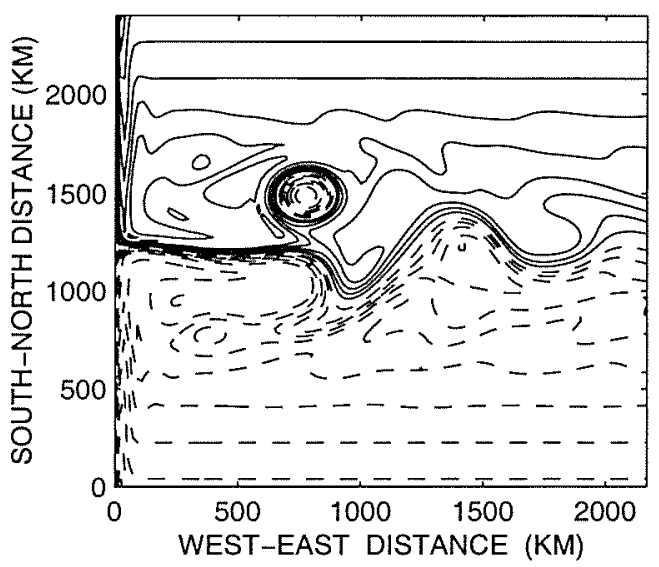

(f)

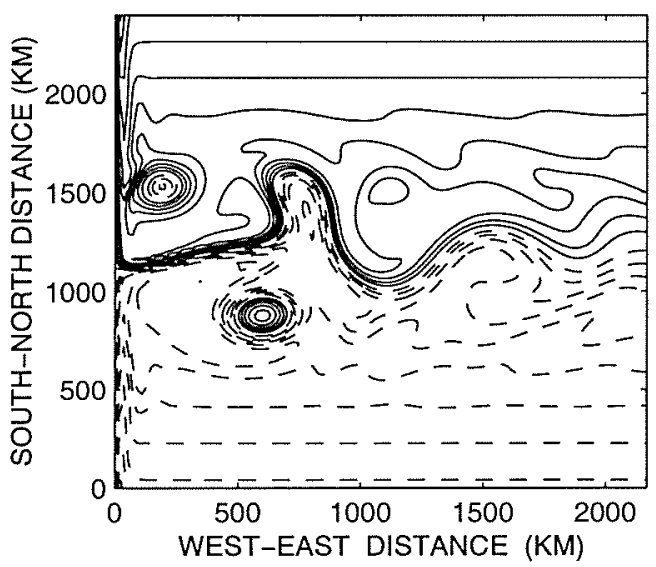

Figure 4-35: Contours of instantaneous streamfunction field and potential vorticity at time $t=1.3$ years $(\mathrm{a}, \mathrm{b}), t=5.2$ years $(\mathrm{c}, \mathrm{d})$ and $t=10.4$ years $(\mathrm{e}, \mathrm{f})$. Time, $t$, is as in Figure 4-34. Parameters: $T_{i n}=16 \mathrm{~Sv}, \mathcal{A}_{6}^{d i m}=8 \cdot 10^{10} \mathrm{~m}^{4} \mathrm{sec}^{-1}$ and $\mathcal{A}_{2}^{d i m}=3.0 \cdot 10^{-8} \mathrm{sec}^{-1}$. The snapshots are presented for the western part of the model domain. 
(a)

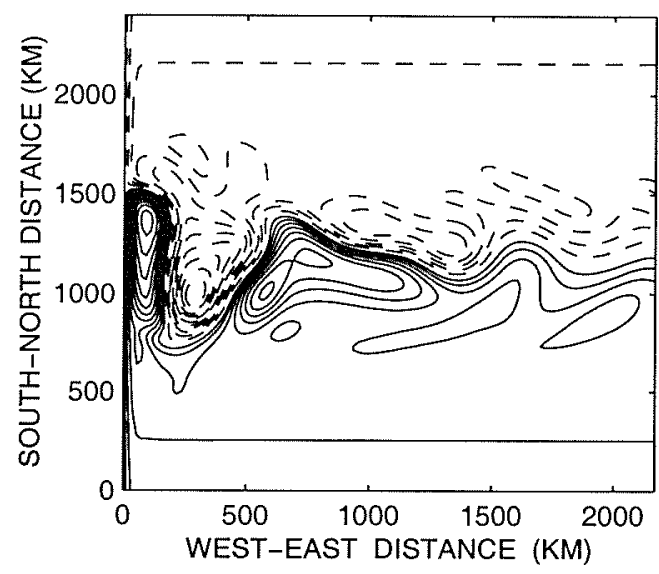

(c)

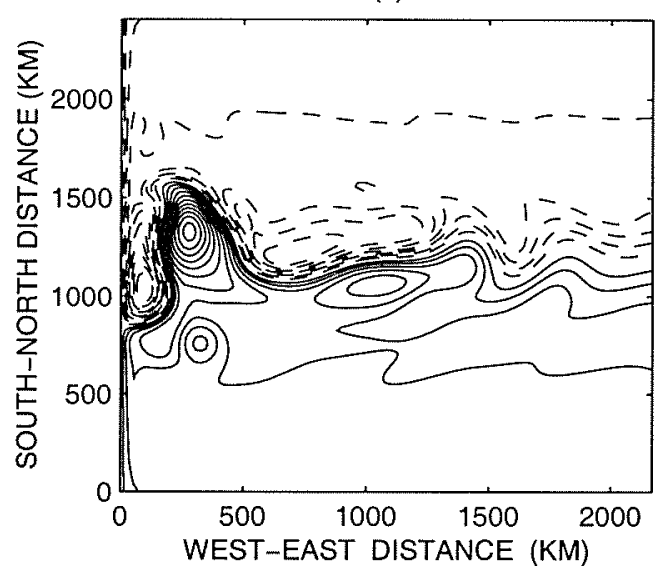

(e)

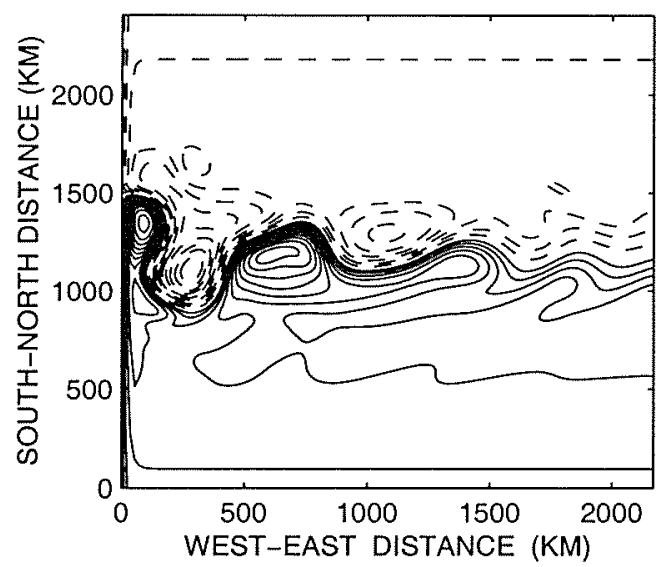

(b)

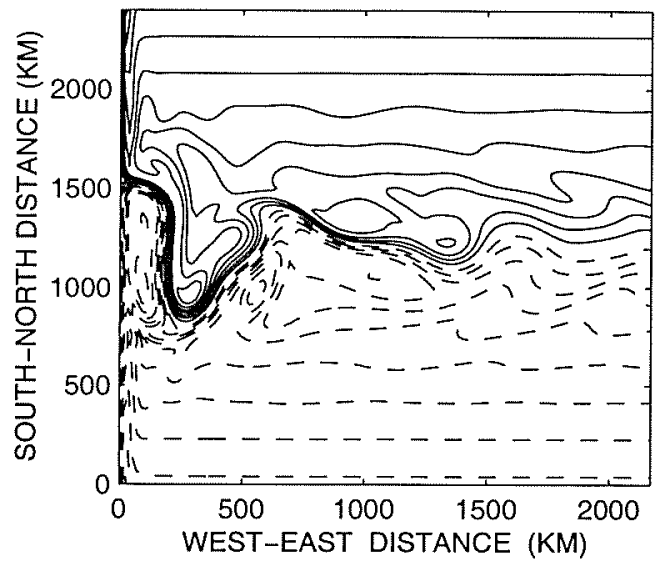

(d)

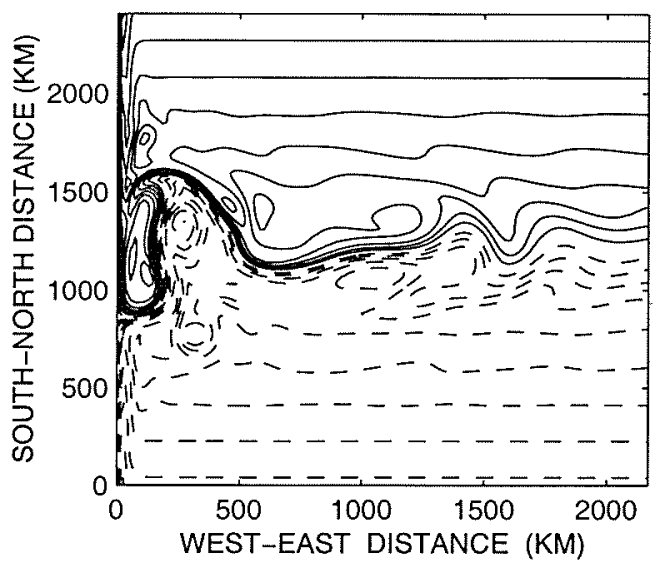

(f)

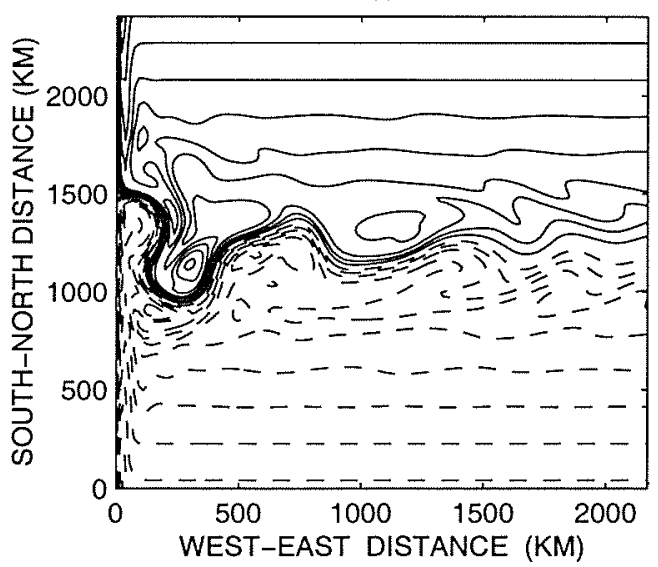

Figure 4-36: Contours of instantaneous streamfunction field and potential vorticity at time $t=13.05$ years $(\mathrm{a}, \mathrm{b}), t=14.35$ years $(\mathrm{c}, \mathrm{d})$ and $t=15.6$ years $(\mathrm{e}, \mathrm{f})$. Time, $t$, is as in Figure 4-34. Parameters: $T_{i n}=16 \mathrm{~Sv}, \mathcal{A}_{6}^{\text {dim }}=8 \cdot 10^{10} \mathrm{~m}^{4} \mathrm{sec}^{-1}$ and $\mathcal{A}_{2}^{\text {dim }}=3.0 \cdot 10^{-8} \mathrm{sec}^{-1}$. The snapshots are presented for the western part of the model domain. 
(a)

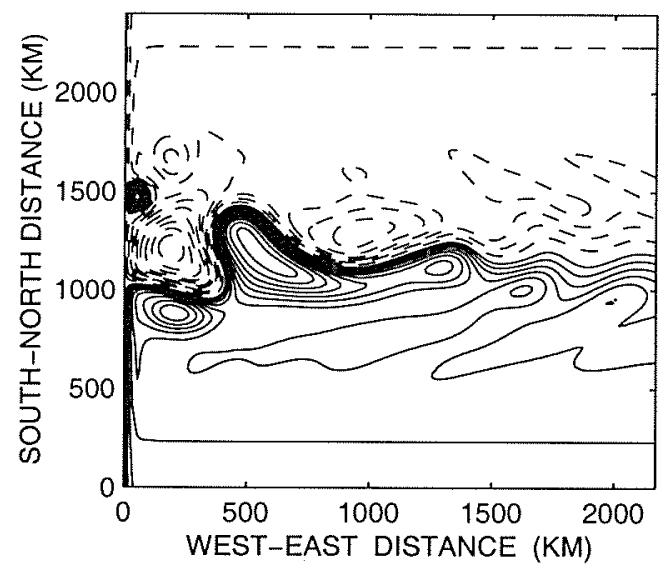

(c)

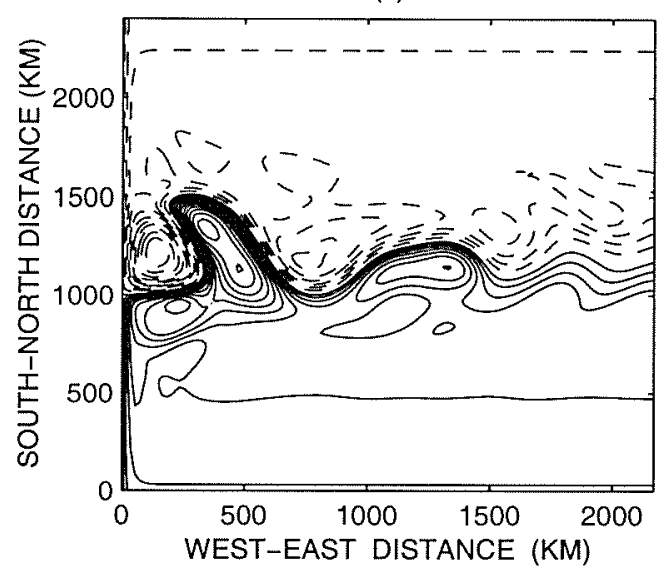

(e)

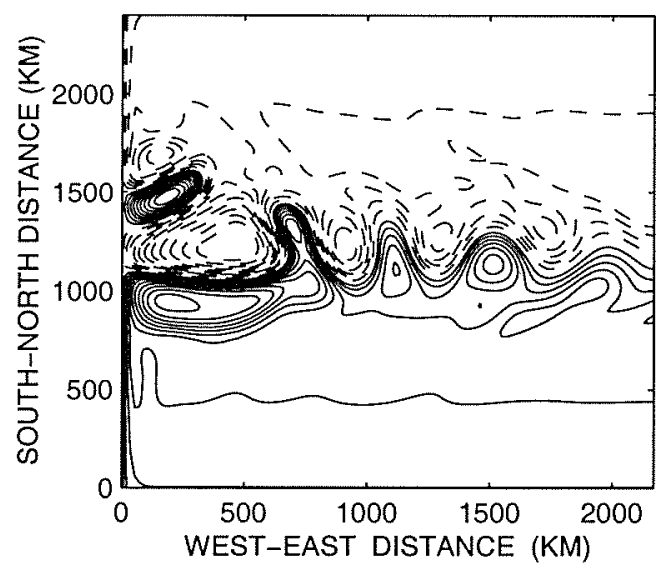

(b)

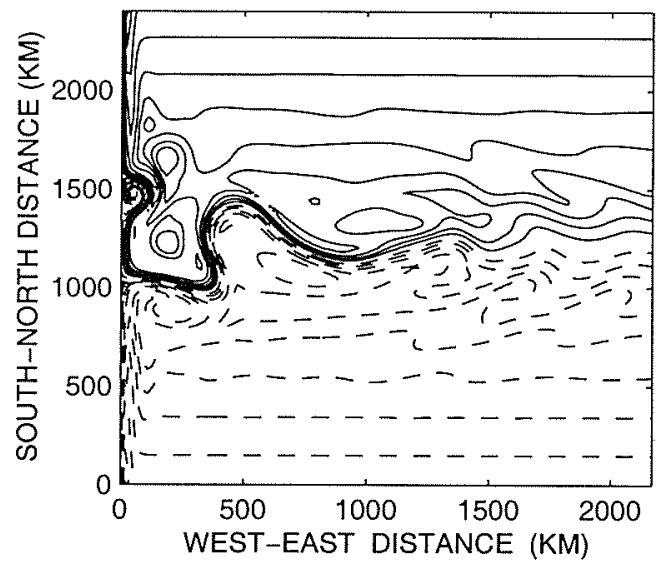

(d)

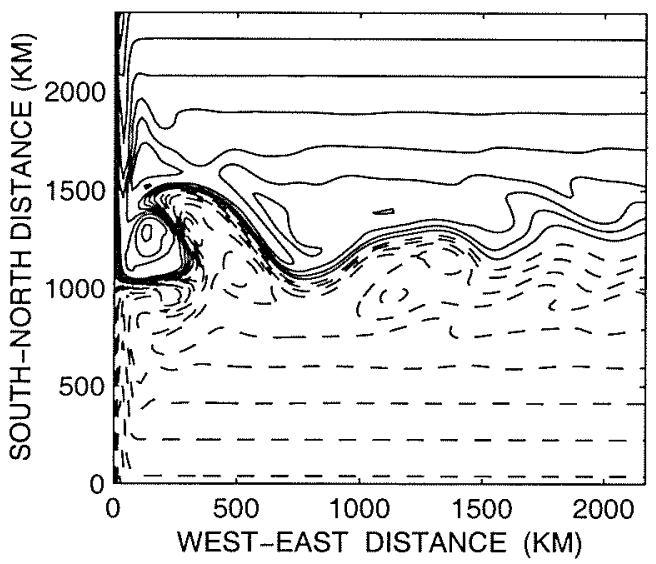

(f)

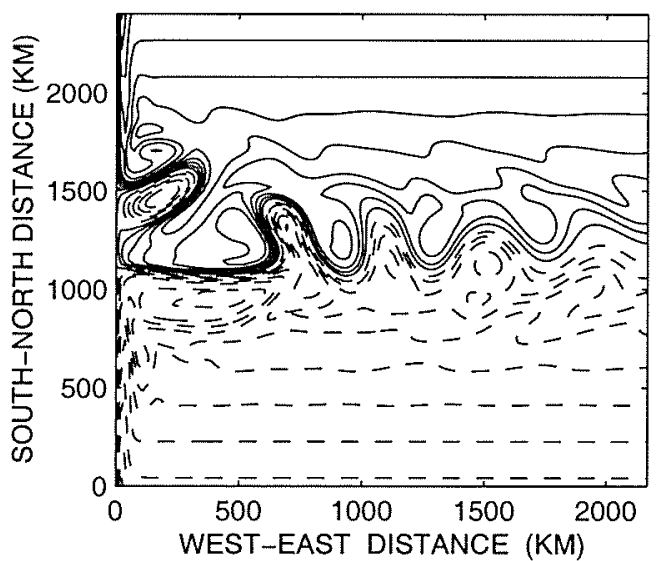

Figure 4-37: Contours of instantaneous streamfunction field and potential vorticity at time $t=18.2$ years $(\mathrm{a}, \mathrm{b}), t=20.8$ years $(\mathrm{c}, \mathrm{d})$ and $t=23.5$ years $(\mathrm{e}, \mathrm{f})$. Time, $t$, is as in Figure 4-34. Parameters: $T_{i n}=16 \mathrm{~Sv}, \mathcal{A}_{6}^{\text {dim }}=8 \cdot 10^{10} \mathrm{~m}^{4} \mathrm{sec}^{-1}$ and $\mathcal{A}_{2}^{\text {dim }}=3.0 \cdot 10^{-8} \mathrm{sec}^{-1}$. The snapshots are presented for the western part of the model domain. 
(a)

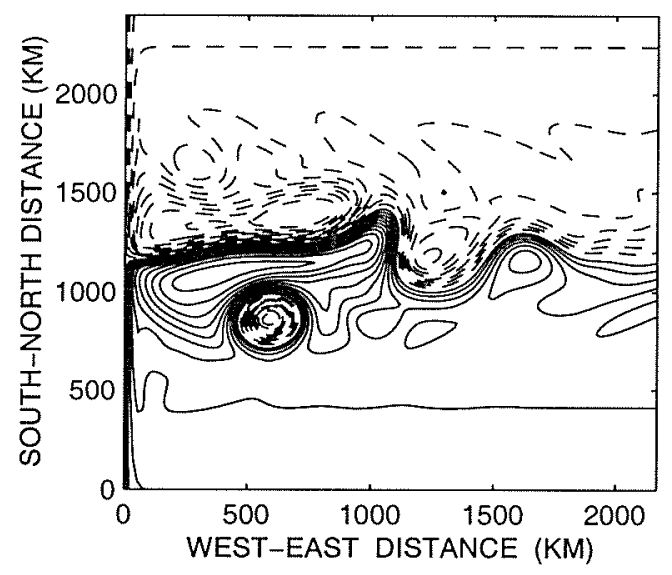

(c)

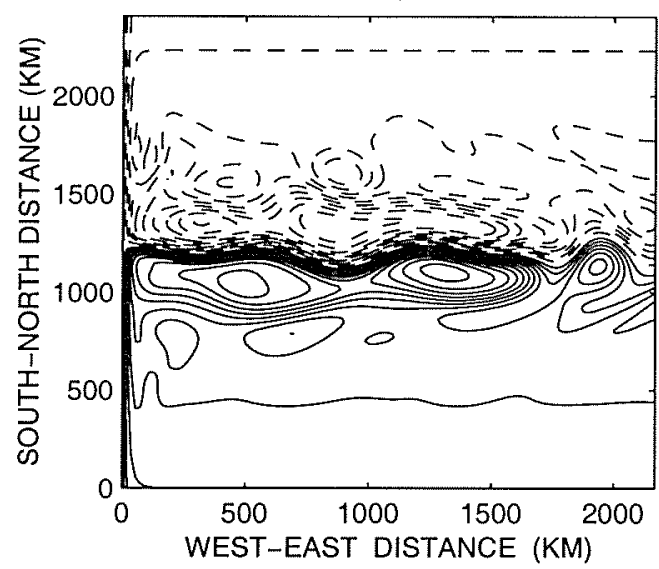

(e)

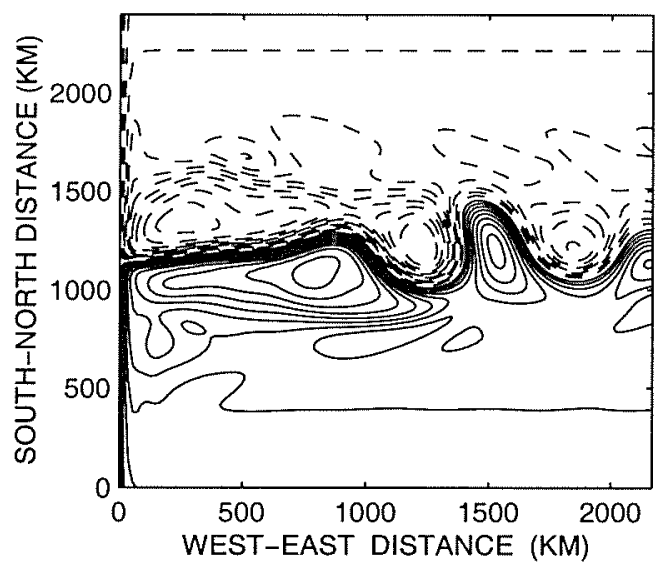

(b)

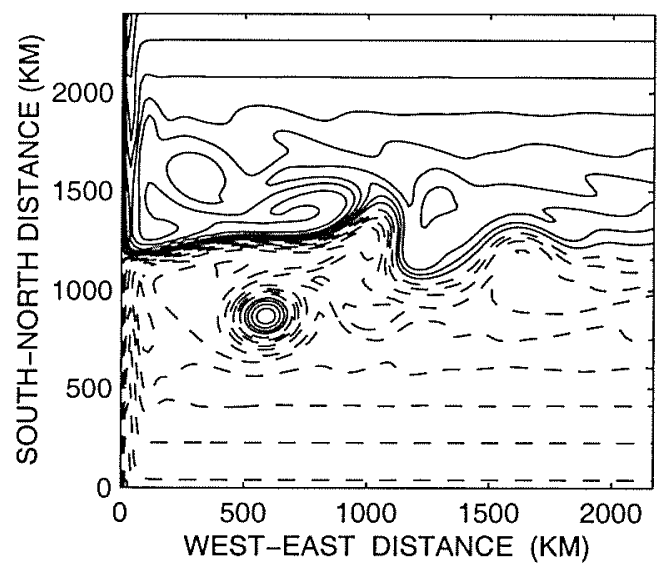

(d)

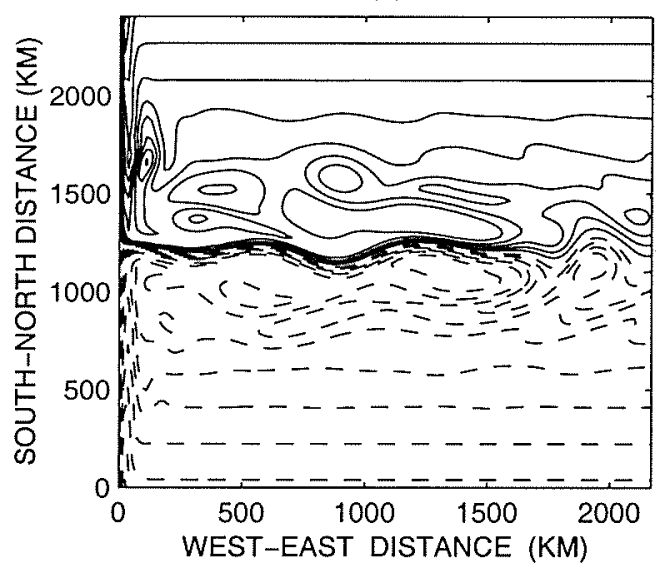

(f)

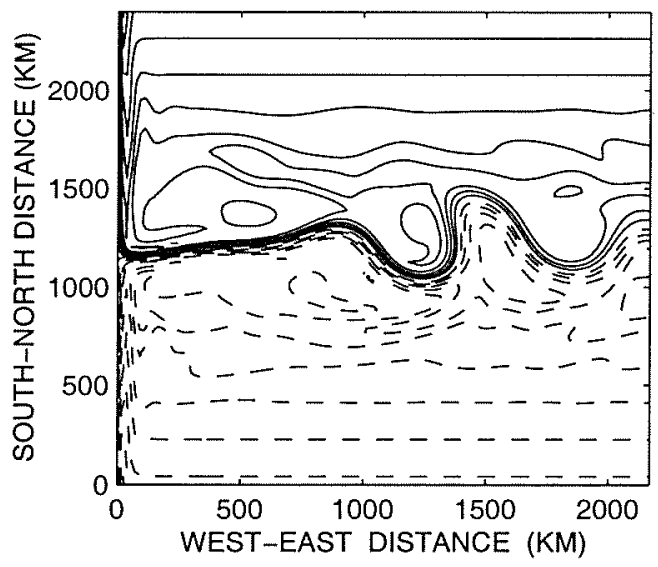

Figure 4-38: Contours of instantaneous streamfunction field and potential vorticity at time $t=26.1$ years $(\mathrm{a}, \mathrm{b}), t=28.7$ years $(\mathrm{c}, \mathrm{d})$ and $t=32.6$ years $(\mathrm{e}, \mathrm{f})$. Time, $t$, is as in Figure 4-34. Parameters: $T_{i n}=16 \mathrm{~Sv}, \mathcal{A}_{6}^{\text {dim }}=8 \cdot 10^{10} \mathrm{~m}^{4} \mathrm{sec}^{-1}$ and $\mathcal{A}_{2}^{\text {dim }}=3.0 \cdot 10^{-8} \mathrm{sec}^{-1}$. The snapshots are presented for the western part of the model domain. 

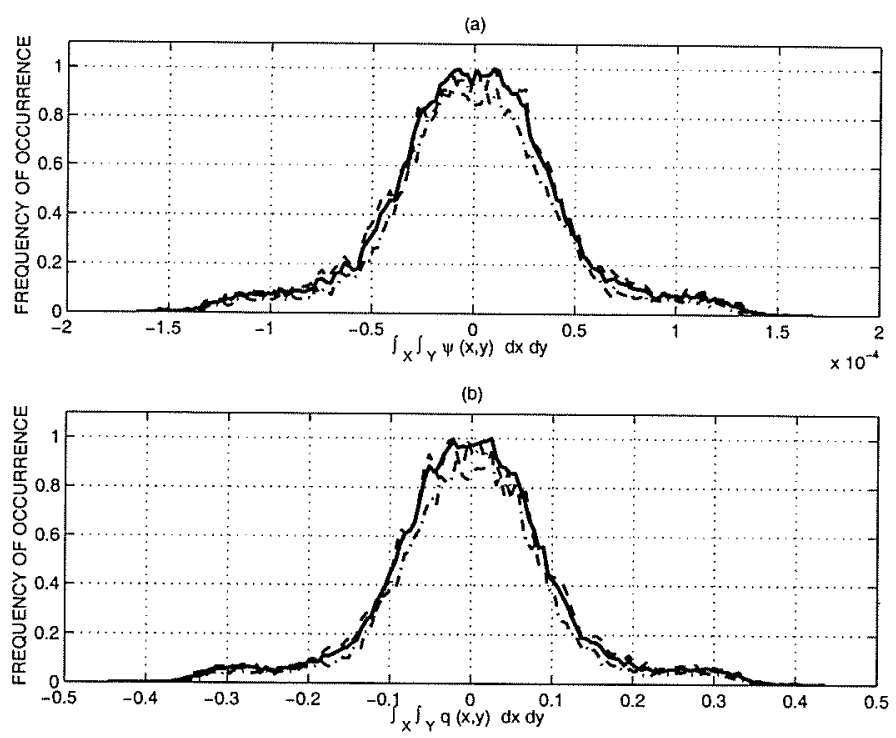

Figure 4-39: Normalized histograms of domain-integrated streamfunction (a, solid) and potential vorticity ( $b$, solid) for the reference run. Dashed line denotes histograms for the first half of the record, dashed-dotted line denotes histograms for the second half of the record. Parameters: $T_{i n}=16 \mathrm{~Sv}, \mathcal{A}_{6}^{\text {dim }}=8 \cdot 10^{10} \mathrm{~m}^{4} \mathrm{sec}^{-1}$ and $\mathcal{A}_{2}^{\text {dim }}=$ $3.0 \cdot 10^{-8} \mathrm{sec}^{-1}$.

\subsubsection{Mass and Potential Vorticity Balances}

Before we describe the mass and potential vorticity balances, let us recall that due to a hydrostatic balance and quasi-geostrophic assumptions, mass is given by the domainintegrated streamfunction field. The present model does not have special numerical procedures which would guarantee conservation of mass and potential vorticity at each time step. However, fixed boundary conditions, providing the balance of geostrophic inflows and outflows, and the quasi-geostrophic equations of motion, allowing only for an order of Rossby number disturbances in the pressure surfaces, permit only small deviations in the domain-integrated streamfunction from its original zero value.

Figure 4-39 shows normalized histograms of the domain-integrated streamfunction and potential vorticity fields. Dashed and dashed-dotted lines present similar histograms based on the first and the second halves of the time series and indicate that the shape of the histograms does not change qualitatively. Therefore, the length 
of the time series is pretty robust. A straight line fitted to the time series gives slope coefficients of $6.1 \mathrm{e}-10$ and 1.1e-6 for the integrals of the streamfunction and potential vorticity fields respectively. Although the realization is finite, the small values of the slope coefficients indicate that integrated mass and potential vorticity do not drift in time, even though the model has no explicit conservation scheme for any of those fields.

QQPLOTs based on the time series of domain-integrated streamfunction and potential vorticity are presented in Figures 4-40a,c. Clearly, there are large deviations from a linear function, which indicates that the corresponding distributions are not normal. The latter is due to the presence of the low energy state. Figure 4-41 shows histograms based on the segments of the original time series of $\int_{x} \int_{y} \psi d x d y$ and $\int_{x} \int_{y} q d x d y$ that were constructed when total energy was either larger than 0.067 (a high energy state, solid line) or smaller than 0.064 (a low energy state, dashed line). The histograms of $\psi$ - and $q$-integrals have normal distributions during the high energy period, since the corresponding QQPLOTs are linear (Figures 4-40b,d). As we saw earlier, during the high energy state, the flow structure is characterized by the well-developed recirculations, and the time mean streamfunction and potential vorticity fields are well defined.

However, during the low energy state, the model displays strong fluctuations associated with large meridional excursions in the separation point. Clearly, when there is significant northward (southward) excursion, the integrated potential vorticity field must be negative (positive). The distributions of $\psi$ - and $q$-integrals during the low energy period are not normal, since there is no well defined time mean and since there is no preferred flow structure. Therefore, the presence of the low energy state is responsible non-normal distribution of the mass and integrated potential vorticity time series. 

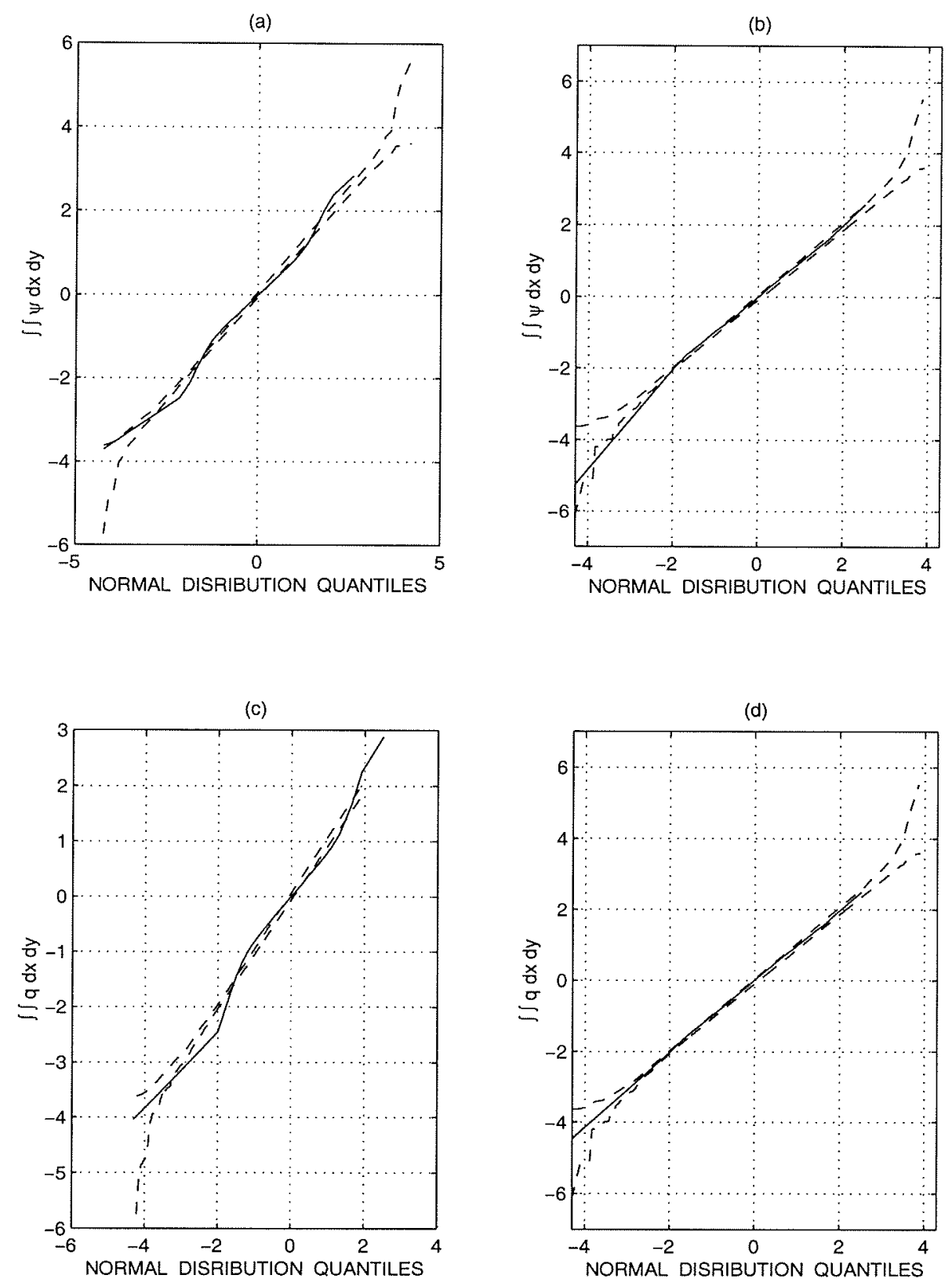

Figure 4-40: (a): QQPLOT of the quantiles of the time series of $\int_{x} \int_{y} \psi d x d y$ vs the quantiles of the randomly generated time series with normal distribution. (b): QQPLOT of the time series of $\int_{x} \int_{y} \psi d x d y$ with the corresponding total energy level greater than 0.067. (c): QQPLOT of the total time series of the domain-integrated potential vorticity. (d): QQPLOT of the domain-integrated potential vorticity time series with the corresponding total energy level greater than 0.067. Dashed line denotes error bars for normal distribution. Parameters: $T_{i n}=16 \mathrm{~Sv}, \mathcal{A}_{6}^{\text {dim }}=8 \cdot 10^{10}$ $\mathrm{m}^{4} \mathrm{sec}^{-1}$ and $\mathcal{A}_{2}^{\mathrm{dim}}=3.0 \cdot 10^{-8} \mathrm{sec}^{-1}$. 

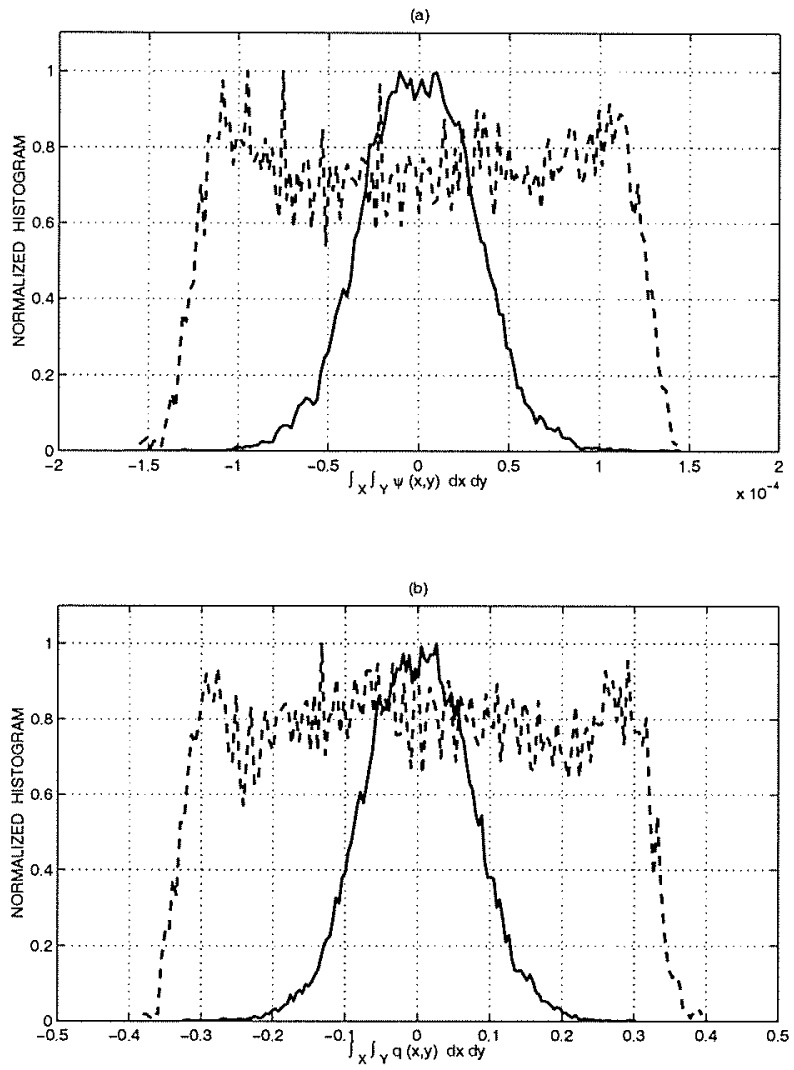

Figure 4-41: Normalized histograms of domain-integrated streamfunction (a) and potential vorticity (b) for the reference run. Dashed line denotes histograms based on the segment of the total time series when total energy level was larger than 0.067 . Dashed line corresponds to the segments when the total energy level was smaller than 0.064. Parameters: $T_{i n}=16 \mathrm{~Sv}, \mathcal{A}_{6}^{\text {dim }}=8 \cdot 10^{10} \mathrm{~m}^{4} \mathrm{sec}^{-1}$ and $\mathcal{A}_{2}^{\text {dim }}=3.0 \cdot 10^{-8} \mathrm{sec}^{-1}$. 


\subsubsection{Transitions Between High and Low Energy States}

To understand the dynamics of transitions between the high and low energy states, the "ensemble average", or composite, time series of the streamfunction field was constructed in the following way. First, we identified all the HL (LH) events. Second, we chose subjectively a "mean" energy level for all transitions as that at the middle of the transition. Third, we extracted 2.5 years of $\psi$-field before and after the model reached this energy value. The latter permitted time series of equal lengths for $\mathbf{H L}$ (LH) events:

$$
\left\{\psi^{k}\left(t_{1}\right), \psi^{k}\left(t_{2}\right), \cdots, \psi^{k}\left(t_{m}\right)\right\}
$$

where $m$ is the length of each time series and $k=1, \cdots, K$ is the index of an individual event. Third, a "mean" HL ( $\mathbf{L H})$ transition time series was constructed as

$$
\left\{\psi\left(t_{1}\right), \psi\left(t_{2}\right), \cdots \psi\left(t_{m}\right)\right\}
$$

where $\psi\left(t_{i}\right)=\frac{1}{K} \sum_{k=1}^{K} \psi^{k}\left(t_{i}\right)$.

Figures 4-42a-b show the "time mean" streamfunction and potential vorticity fields calculated from the time-series defined by (4.12) for the HL-transitions. One should remember that since transitions are fundamentally non-steady, the term "time mean" should be used with caution. The spatial structures of the fields are similar to those of the low energy periods; the western boundary currents appear separating 300 $\mathrm{km}$ north and south of the middle of the domain, and weak recirculations are shifted eastward. The above mean state does not reflect the true structure of the flow, when the interaction of large-amplitude, westward propagating meanders with the western wall produces large meridional excursions in the separation point. The structure of the time mean perturbation fields (Figure 4-43) reveals that dominant eddy processes and conversions occur along the western boundary.

During the LH-transitions, the symmetry of the inflow near the collision point is restored, and the time mean streamfunction and potential vorticity (Figures 4$42 \mathrm{c}-\mathrm{d}$ ) are characterized by the $1000-\mathrm{km}$ long recirculating gyres. The size of the 

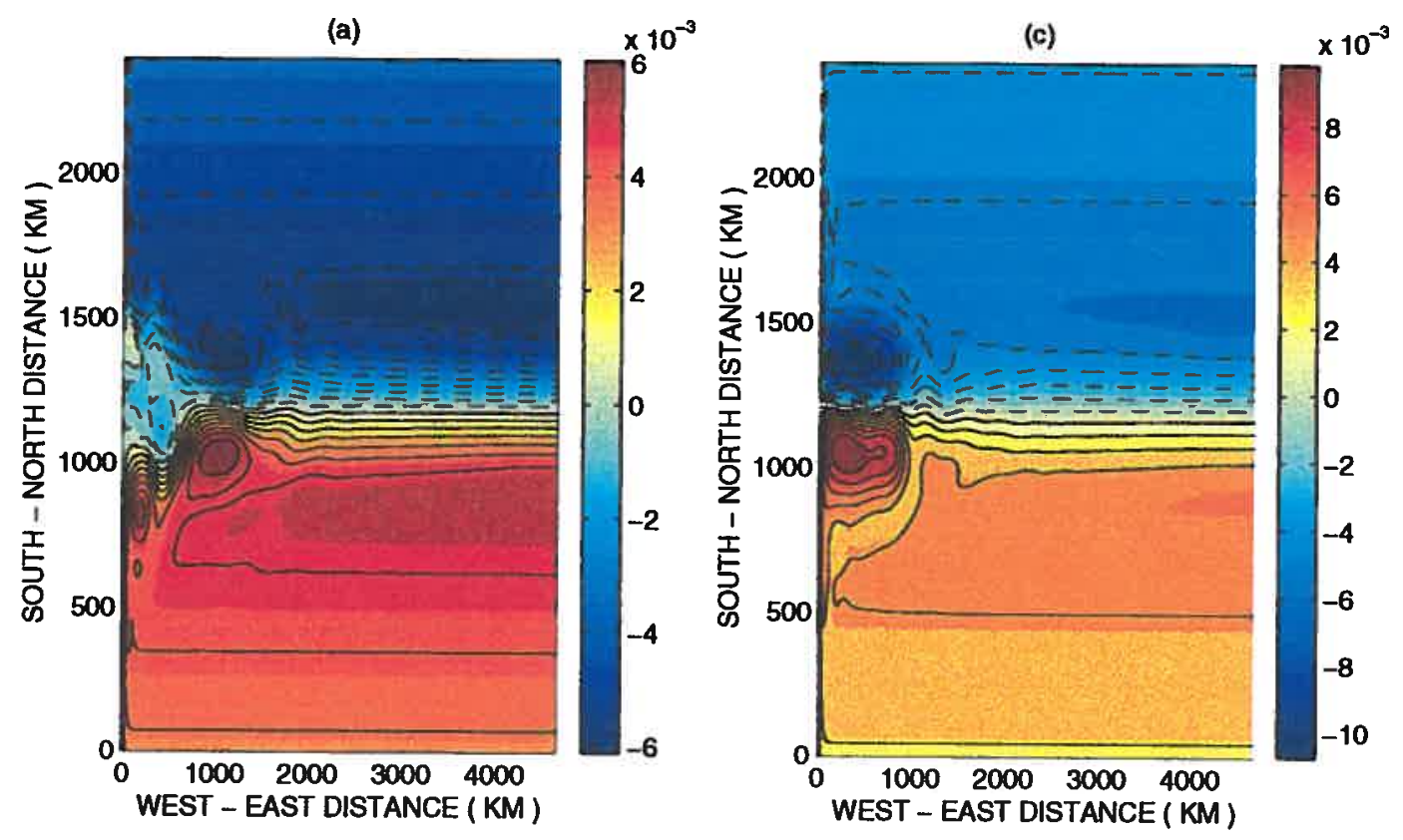

(b)
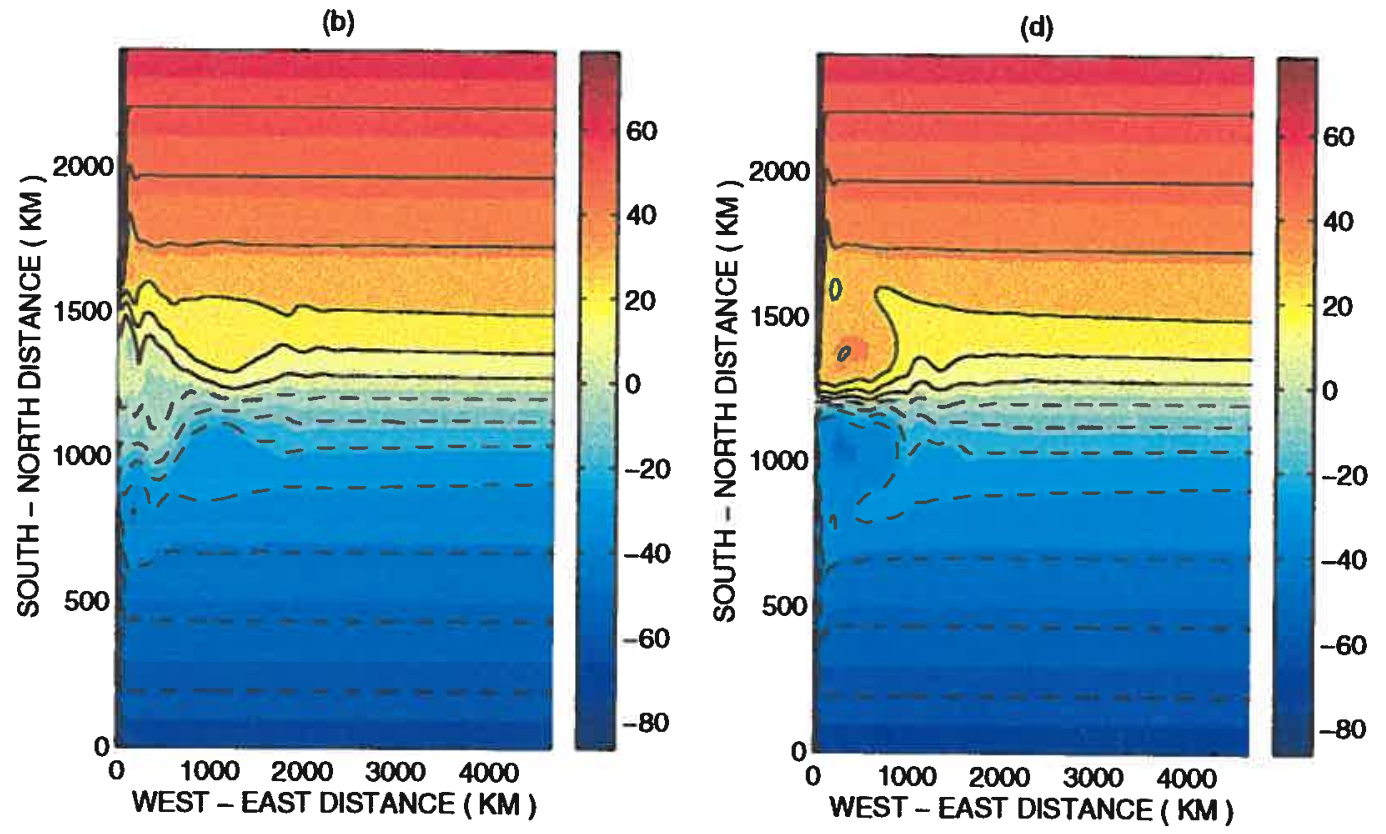

Figure 4-42: Contours of mean streamfunction and potential vorticity for HLtransition (a,b) and LH-transition (c-d). Parameters: $T_{i n}=16 \mathrm{~Sv}, \mathcal{A}_{6}^{\text {dim }}=8 \cdot 10^{10}$ $\mathrm{m}^{4} \mathrm{sec}^{-1}$ and $\mathcal{A}_{2}^{\text {dim }}=3.0 \cdot 10^{-8} \mathrm{sec}^{-1}$. 
(a)

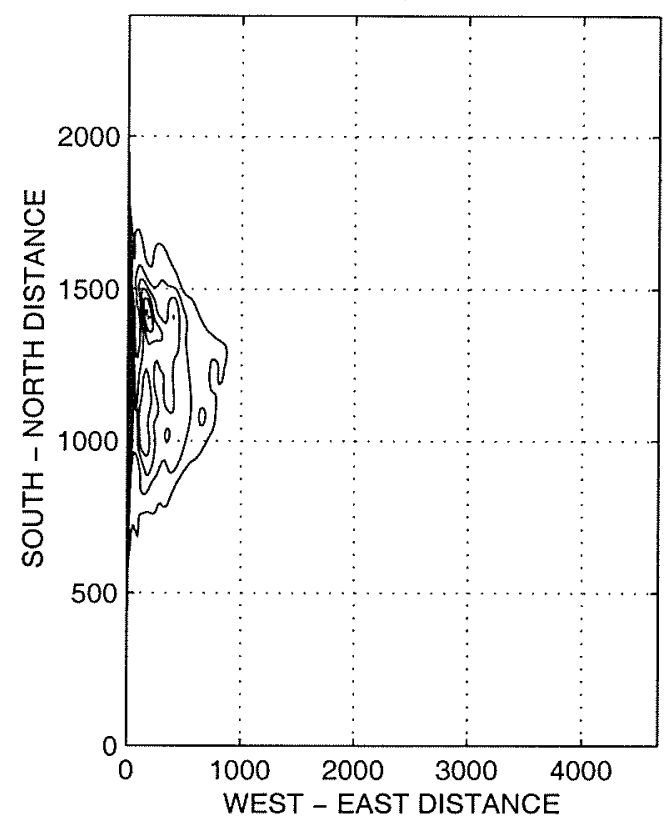

(c)

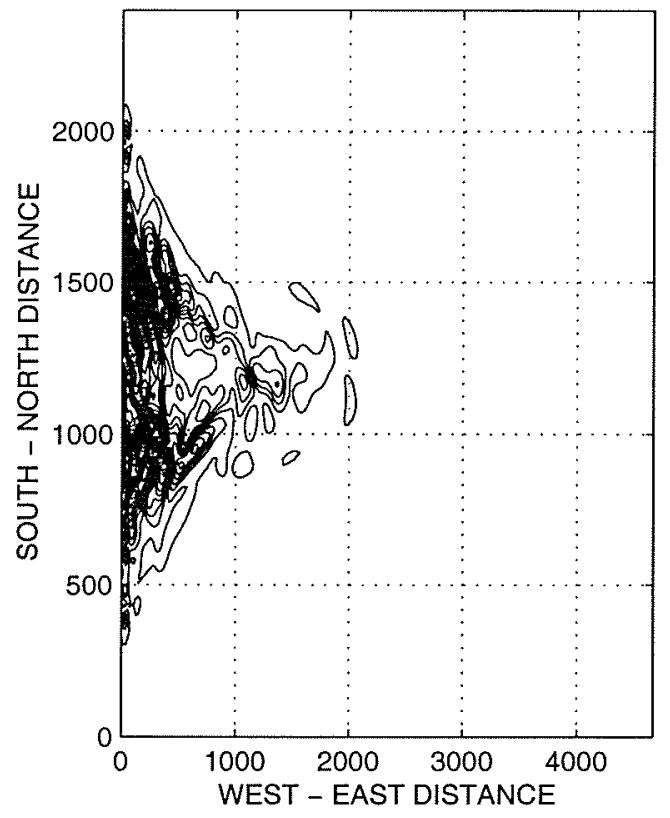

(b)

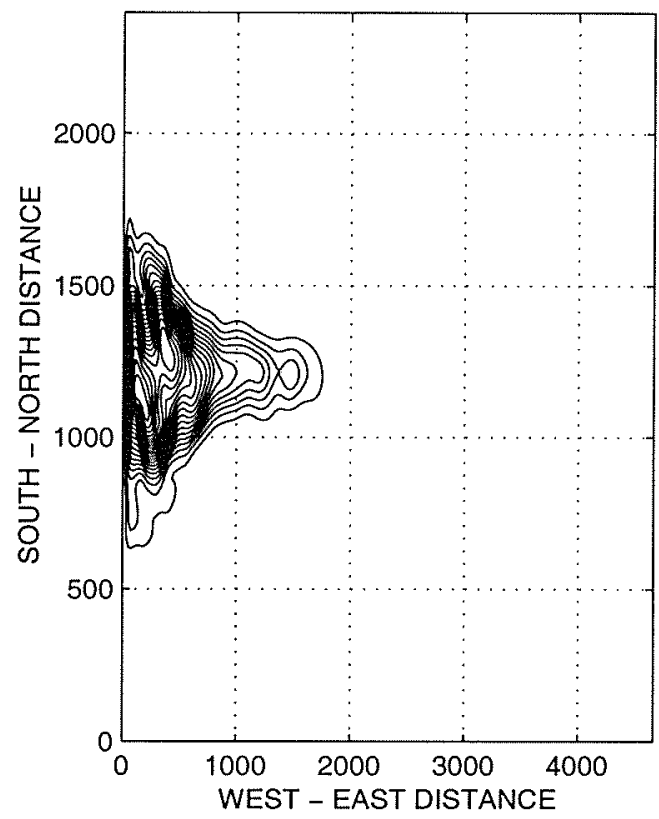

(d)

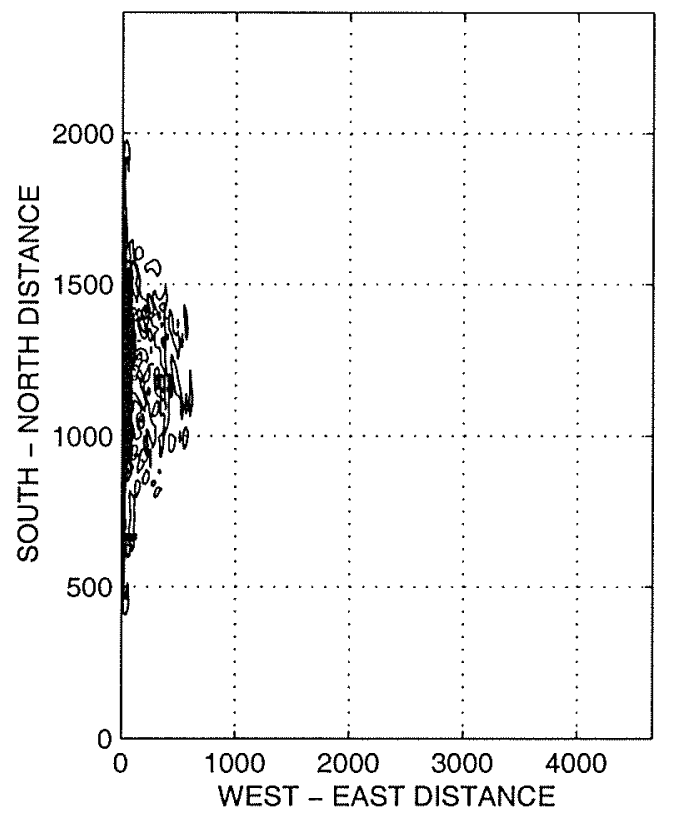

Figure 4-43: Time mean perturbation kinetic energy density (a), perturbation potential energy density (b), barotropic conversion energy density (c) and perturbation energy dissipation (d) during the HL-transition. Parameters: $T_{\text {in }}=16 \mathrm{~Sv}$, $\mathcal{A}_{6}^{\operatorname{dim}}=8 \cdot 10^{10} \mathrm{~m}^{4} \mathrm{sec}^{-1}$ and $\mathcal{A}_{2}^{\operatorname{dim}}=3.0 \cdot 10^{-8} \mathrm{sec}^{-1}$. 
(a)

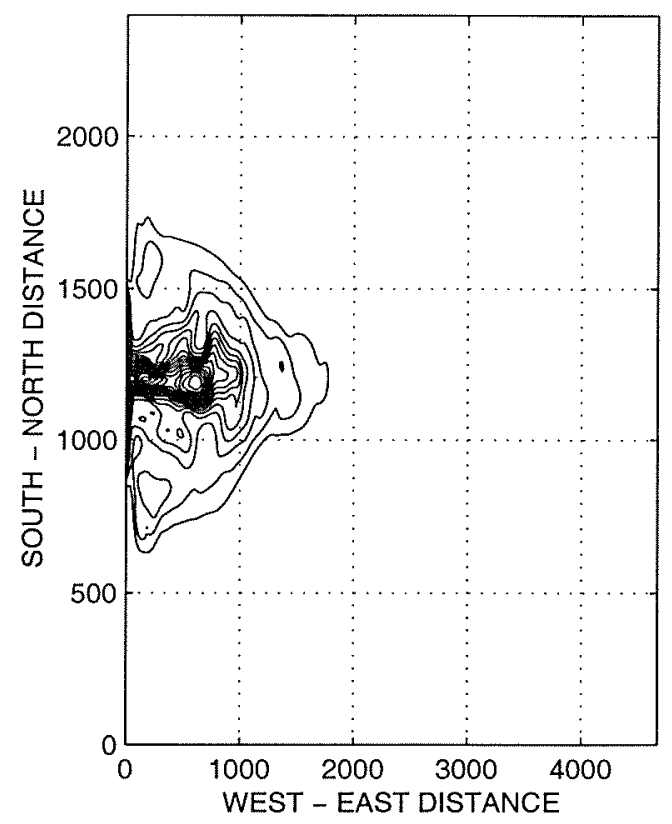

(c)

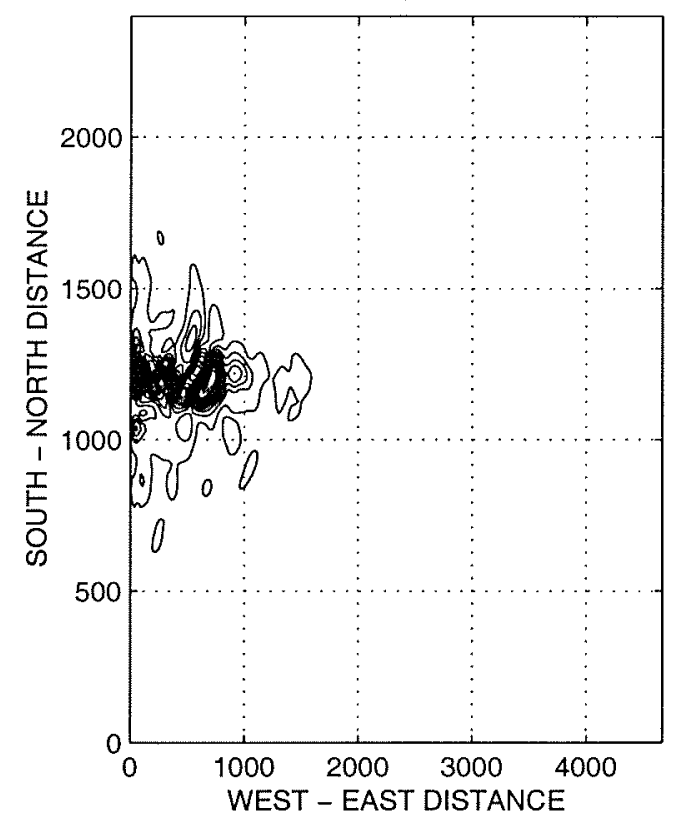

(b)

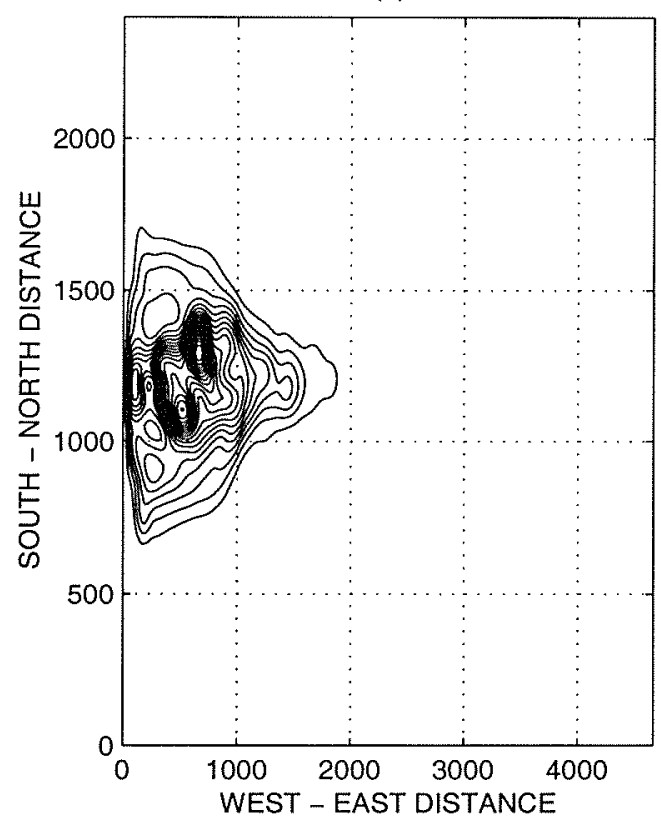

(d)

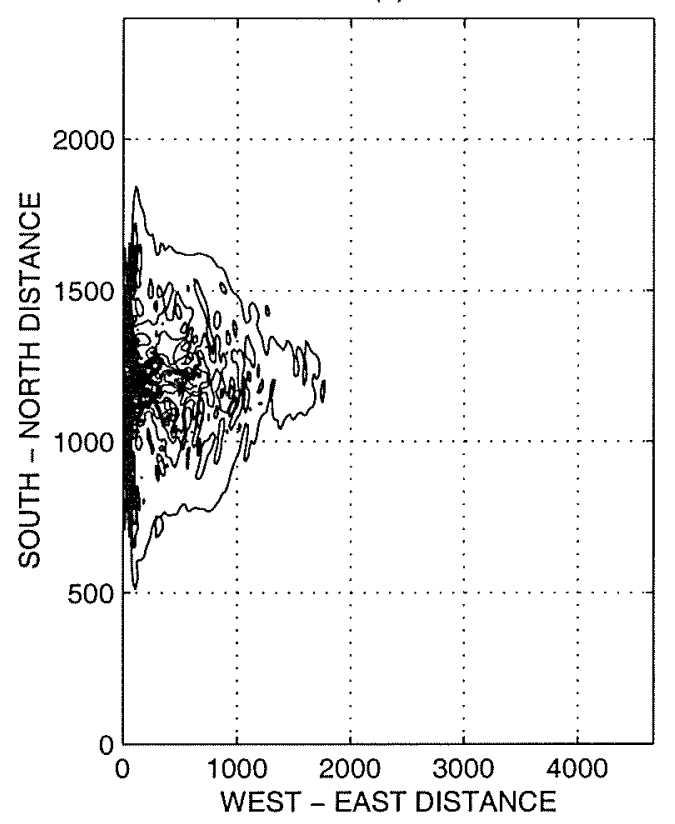

Figure 4-44: Time mean perturbation kinetic energy density (a), perturbation potential energy density (b), barotropic conversion energy density (c) and perturbation energy dissipation (d) during the LH-transition. Parameters: $T_{\text {in }}=16 \mathrm{~Sv}$, $\mathcal{A}_{6}^{\text {dim }}=8 \cdot 10^{10} \mathrm{~m}^{4} \mathrm{sec}^{-1}$ and $\mathcal{A}_{2}^{\text {dim }}=3.0 \cdot 10^{-8} \mathrm{sec}^{-1}$. 
recirculations increases from 500 to $1800 \mathrm{~km}$ during the transition events. The maxima in the eddy density fields (Figure 4-44) are aligned with the axis of the jet, and the conical shape of the perturbation energy density indicates the importance of rings in flattening of the potential vorticity front. The model does not quite reach the steady antisymmetric solution, since the amplitude of the eddy field starts increasing when the recirculations become large.

Therefore, the dynamics of transitions from the high to low energy states are drastically different from those of the low to high energy transitions. In the first case, the eddy field becomes very strong, giving rise to the large amplitude, westward propagating meanders, which produce large meridional excursion in the separation

point and strong northward and southward propagating rings. In the second case, the eddy field weakens, and the advection of potential vorticity by the western boundary currents restores the flow symmetry by quickly inducing large symmetric recirculations. Figures 4-45 and 4-46 summarize the energy conversion during the transition events. When reading the diagrams, one should remember that the flow is not in a steady state during the transition events, and therefore the energy terms do not balance each other. Both eddy kinetic and eddy potential energies are of the same order of magnitude as the "time mean" flow kinetic energy during the HL-transition. The positive sign of the domain-integrated barotropic conversion rate indicates that eddy energy grows at the expense of the mean flow energy. During the LH-transition, the eddies release energy back to the mean flow, which is associated with the negative barotropic conversion integral.

\subsection{Discussion of Nonsymmetric Inflow Conditions}

In this study, we used symmetric inflow and outflow conditions, which were constructed to simulate a boundary with the Sverdrup interior forced by symmetric wind forcing. It would be interesting to see whether low frequency variability and mul- 


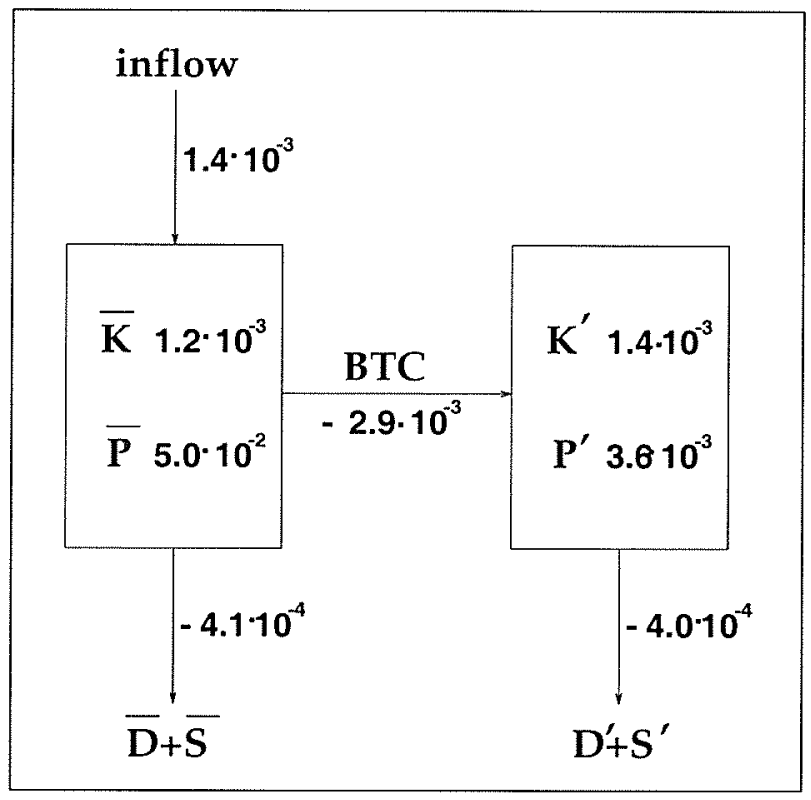

Figure 4-45: Energy diagram for synthetic HL-transition. Parameters: $T_{\text {in }}=16 \mathrm{~Sv}$, $\mathcal{A}_{6}^{\text {dim }}=8 \cdot 10^{10} \mathrm{~m}^{4} \mathrm{sec}^{-1}$ and $\mathcal{A}_{2}^{\text {dim }}=3.0 \cdot 10^{-8} \mathrm{sec}^{-1}$.

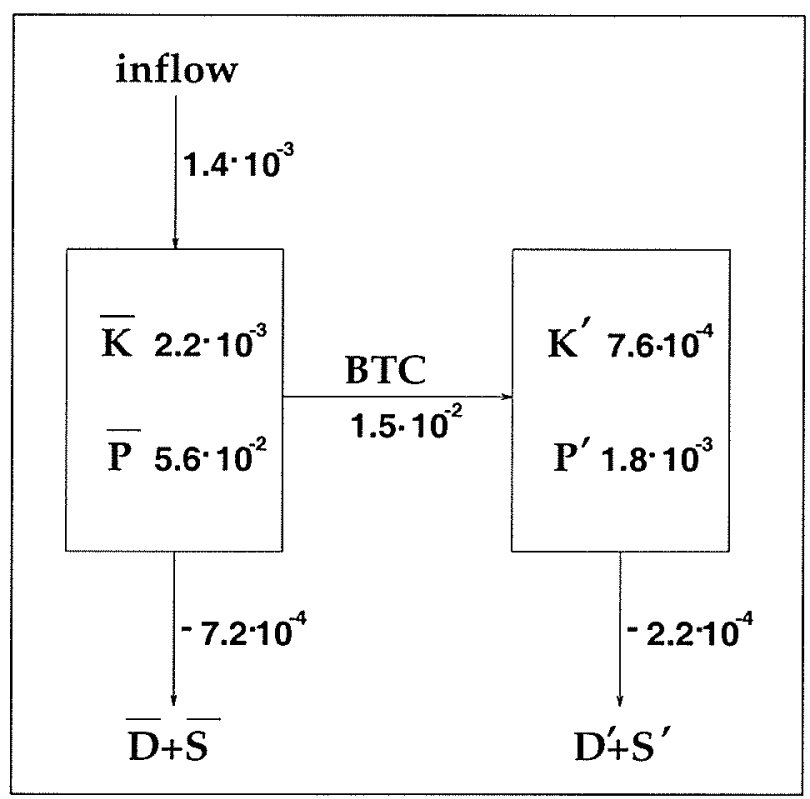

Figure 4-46: Energy diagram for synthetic LH-transition. Parameters: $T_{i n}=16 \mathrm{~Sv}$, $\mathcal{A}_{6}^{\text {dim }}=8 \cdot 10^{10} \mathrm{~m}^{4} \mathrm{sec}^{-1}$ and $\mathcal{A}_{2}^{\text {dim }}=3.0 \cdot 10^{-8} \mathrm{sec}^{-1}$. 
tiple dynamical regimes are still possible if the strength of the inflows through the meridional boundaries were not equal. In this case, the model solution might depend strongly on the streamfunction and potential vorticity distributions on the eastern boundary, as explained below.

One way to illustrate possible dependence of the model solution on the eastern boundary outflow when the meridional inflows are of different strengths is to calculate the total potential vorticity input through the model domain. Assume that the flow through the southern part of the domain is weaker than that through the northern part of the domain, i.e. $\psi_{s}=-\alpha \psi_{n}$, where $0<\alpha<1, \psi_{s}=\psi\left(x, y_{s}\right)$ and $\psi_{n}=\psi\left(x, y_{n}\right)$. The potential vorticity distribution on the southern boundary, $q_{s}=q\left(x, y_{s}\right)$, can be then expressed in terms of the potential vorticity distribution on the northern boundary, $q_{n}=q\left(x, y_{n}\right)$, as

$$
q_{s}=\beta y_{s}+\frac{\partial^{2} \psi_{s}}{\partial x^{2}}-\gamma^{2} \psi_{s}=(\alpha-1) \beta y_{n}-\alpha q_{n}
$$

The total potential vorticity input into the model domain is given by

$$
\begin{aligned}
\oint_{\bar{\Omega}} q \mathbf{u} \cdot \mathbf{n} d \bar{\Omega}= & \int_{x_{w}}^{x_{e}}\left[\alpha(\alpha-1) \beta y_{n} \frac{\partial \psi_{n}}{\partial x}+\left(1-\alpha^{2}\right) q_{n} \frac{\partial \psi_{n}}{\partial x}\right] d x- \\
& \int_{y_{s}}^{y_{n}} q_{e} \frac{\partial \psi_{e}}{\partial y} d y
\end{aligned}
$$

where $q_{e}=q\left(x_{e}, y\right)$ and $\psi_{e}=\psi\left(x_{e}, y\right)$ are potential vorticity and streamfunction on the eastern boundary, $\mathbf{u}=(u, v)^{T}$ is a geostrophic velocity vector on the model boundary, $\bar{\Omega}$, and $\mathbf{n}$ is a vector normal to the boundary. In the case of symmetric inflow conditions, the coefficient $\alpha$ is equal to one, and therefore the first integral of the right hand side is zero. If $\psi_{e}$ is an odd function, the second integral on the right hand side is also zero. Therefore, total potential vorticity input is zero if the inflow and outflow conditions are symmetric. This case was of our primary interest.

For a nonzero $\alpha$, the value of the first integral is negative. Hence, in order for the total potential vorticity flux to vanish, the outflow on the eastern wall has to balance potential vorticity input through the meridional boundaries. If the outflow is 
such that the total $q$ - input is non zero, it may lead to enhanced potential vorticity transfer across the middle of the domain, and therefore it might significantly affect the interior solution.

Another reason, why the model might display different behavior, is that steady state solutions might change significantly when the inflows are chosen to be nonsymmetric. In the present study, we did not attempt to find such solutions. However, as we showed for the symmetric inflows, steady state solutions are important for the existence of multiple dynamical regimes. Therefore, one might expect that the change in the structure of steady solutions might lead to the change in the character and the occurrence of multiple states.

For comparison, McCalpin and Haidvogel [25] showed that for strongly asymmetric wind, the reduced-gravity model was not able to reach the high energy state and instead stayed in the low energy regime. The absence of the high energy state may have been due to "correlation" phenomena (Scott and Straub [34]) between the global structures of the wind forcing and the interior solution. In our boundary-forced model, such correlations are not possible. Hence, compared to the wind-driven model, the colliding jets model might display stronger low frequency variability in the case of nonsymmetric inflow conditions, if the steady states are close to each other in the phase space.

\subsection{Summary and Discussion}

In this Chapter, we found steady antisymmetric solutions of the reduced-gravity, colliding jets model. For a wide range of interfacial friction coefficients, the steady flow consisted of an eastward jet flanked by two recirculating gyres. For a set of chosen

parameters, the zonal extent of recirculations and the total energy level increased with a decrease in the interfacial friction coefficient. 
The initial value technique was used to calculate the exponentially growing solutions of the antisymmetric states. The amplitude of the unstable mode closely resembled that of the unstable solution of the wind-driven reduced-gravity model of Primeau [29] and consisted of four strong cells sitting on the axis of the jet between the recirculating gyres and weaker cells in the recirculation regions. The e-folding time varied between 4.8 years and 417 days. With the growth in the instability amplitude, the linear growth halted due to nonlinear interactions of the cells. That interaction led to formation of rings in the area where the jet widens.

Fully nonlinear calculations showed that with an increase in the value of the interfacial friction coefficient, the model became weakly unstable and displayed a unique dynamical regime corresponding to an eastward jet with two recirculating gyres. For near critical values of the friction coefficient, the eddy field was dominated by the eastward and westward propagating meanders of small amplitudes. Rings were weak and therefore dissipated during their propagation through the recirculation regions. The energy level and the flow structure were close to those of the steady antisymmetric solution.

For smaller values of the interfacial friction coefficient, the increased nonlinearity led to low frequency variability associated with changes in the zonal extent of the recirculating gyres. Formation of strong rings in the area east of the recirculations and their subsequent westward propagation through the gyres provided intense potential vorticity mixing across the front and in the area of the recirculations. Rings were strong enough not to be completely dissipated in the recirculations; upon reaching a western boundary, they rejoined the eastward jet. Therefore, the model exhibited periods of large recirculations and weak eddy activity, when the model trajectory was in the vicinity of the antisymmetric fixed point, and periods of small recirculations and strong meander and ring activity.

With further decrease in the interfacial friction, the model displayed two distinct energy states. A high energy state had strong variability associated with well- 
developed recirculations of various zonal extents described in the above paragraph. The low energy state corresponded to a flow regime where the separation point of the eastward jet exhibited large meridional excursions. Weak recirculations existed west of $1000 \mathrm{~km}$ only in the time mean sense. The strong eddy field was dominated by large amplitude, westward propagating meanders whose interaction with the western boundary current produced strong meridionally propagating rings. In comparison, McCalpin and Haidvogel [25] identified three preferred dynamical regimes each with a distinct energy level. Although the high and the medium regime resembled the variability associated with the high energy state of the regional model, the low energy state was quite different from the one of the regional model. This was partly because the outflow conditions of the colliding jets model forced the eastward jet to stay coherent, and therefore the model never reached the point when the jet disappears almost right after separation, as in a low energy state of the wind-driven model.

For low values of the interfacial friction coefficient, the flow stayed in the dynamical regime described as a low energy state in the above paragraph. The amplitude of the eddy field was very strong, and small and weak recirculations existed only in the time mean sense.

In a regional colliding jets model, the role of wind forcing was reduced to prescribing a particular distribution of streamfunction and potential vorticity on the open boundaries. The local nature of such forcing allowed isolating the role of internal barotropic dynamics of the jet and its interaction with the western boundary current as mechanisms responsible for the existence of low frequency variability and multiple dynamical regimes. The numerical experiments with the regional model showed that low frequency variability was an inherent part of the dynamics where nonlinearities were not too weak or too strong. The presence of the western wall was essential, since no low frequency variability or multiple dynamical regimes were observed in a barotropic, zonally asymmetric model of Jayne et al [18], which was forced by an 
unstable zonal jet on the open western boundary and a stable zonal jet on the open eastern boundary.

One of the most important properties of the regional model was a constant energy input, which was given by the boundary integrated energy flux vector. In comparison, in the wind-driven models, the forcing had global character, and the energy input strongly depended on the solution form. Scott and Straub [34] discussed a correlation mechanism between symmetric wind-forcing and a geostrophic current which led to ability of steady antisymmetric solutions to extract more energy than nonsymmetric solutions. The experiments with various wind asymmetry parameter in the wind-driven model of McCalpin and Haidvogel [25] showed that for highly asymmetric wind forcing, the model was not able to reach high energy states. Since the regional model did not have any mechanism that would help to restore the symmetry of the solution, it was harder for the model trajectory to reach and to stay in the vicinity of the antisymmetric steady solution.

The reduced-gravity model had only the mechanism of barotropic instability, and therefore the question remains whether the presence of baroclinic instability could significantly affect low frequency variability. The next Chapter addresses this question in a framework of a two layer, colliding jets model.

In the present study, we used a free slip boundary condition on the western boundary. As Berloff and McWilliams [3] showed, the presence of a no-slip boundary condition tends to destabilize western boundary currents. Eddies produced by such currents can be advected towards the middle of the domain and may interact with rings that propagate towards the western wall from the model's interior. Hence, the nature of low frequency variability and of multiple dynamical regimes might change with the use of no-slip boundary condition due to more complicated dynamics of the western boundary current. This problem, however, was not addressed in the present thesis. 


\section{Chapter 5}

\section{Results for a Two Layer Colliding Jets Model}

\subsection{Introduction}

In this Chapter, we present the results of the numerical experiments with a two-layer, colliding jets model, which includes the mechanisms of both barotropic and baroclinic instabilities. The question is how the latter affects the existence and the character of low frequency variability and whether multiple dynamical regimes are still possible.

As we showed in Chapter 2, baroclinic instability is more efficient than barotropic instability in inducing westward flows during the spin down of a quasi-geostrophic, two-layer, zonally symmetric jet. In strongly baroclinic flows, it was eddy heat fluxes that drove the lower layer recirculations through the residual meridional circulation. For strongly barotropic flows, the mechanisms were nonlinear eddy-eddy and eddymean flow interactions that produced weak westward flows. As we showed in the previous Chapter, the dynamics of rings was also crucial for the existence of multiple dynamical regimes in the reduced-gravity, colliding jets model. The mechanism of baroclinic instability associated with the energy transfer from the vertical shear to the eddy field might be considered as an additional "degree of freedom" for the 
two-layer colliding jets model and therefore may substantially affect the character of the low frequency variability and the existence of multiple dynamical regimes.

The formulation of the model and its governing equations are presented in section 3.2.1. The dissipation terms are given by $\mathcal{D}_{1}=-\mathcal{A}_{6} \nabla^{6} \psi_{1}$ and $\mathcal{D}_{2}=-\mathcal{A}_{2} \nabla^{2} \psi_{2}-$ $\mathcal{A}_{6} \nabla^{6} \psi_{2}$, where $\mathcal{A}_{6}$ is a biharmonic diffusion coefficient and $\mathcal{A}_{2}$ is a bottom friction coefficient.

\subsection{Comments on Strong Bottom Friction}

As we shall see, bottom friction in the two-layer model physically plays an opposite role to that of interfacial friction in the reduced-gravity model; as bottom friction increases, the upper layer flow behaves as that of the reduced-gravity model with weak interfacial friction. The physics can be illustrated by the following scaling argument. Suppose that bottom friction is strong in the two-layer model, and therefore the amplitude of the lower layer streamfunction, $\psi_{2}$, is small. Assume also that the lower layer depth is large, so that the lower layer Froude number is small. With the above assumptions, the dominant balance in the lower layer potential vorticity equation is between the time changes in the upper layer streamfunction and the bottom friction operator applied to the lower layer streamfunction:

$$
F_{2} \frac{\partial \psi_{1}}{\partial t} \sim-\mathcal{A}_{2} \nabla^{2} \psi_{2}
$$

Rewriting the upper layer potential vorticity field as $\Pi_{1} \sim \beta y+\nabla^{2} \psi_{1}-F_{1} \psi_{1}$, the governing equation for the upper layer flow becomes:

$$
\frac{\partial \Pi_{1}}{\partial t}+J\left(\psi_{1}, \Pi_{1}\right) \sim-\mathcal{A}_{6} \nabla^{6} \psi_{1}+\mathcal{S}_{1}+\mathcal{A}_{2}^{-1}\left\{F_{1} F_{2} \nabla^{-2} \frac{\partial^{2}}{\partial t^{2}}\right\} \psi_{1}
$$

The last term in the above equation appears as frictional forcing exerted by the lower layer flow on the upper layer flow. The bottom friction coefficient enters with the power of -1 , which indicates that with an increase in $\mathcal{A}_{2}$, the upper layer flow dynamics becomes similar to that of a reduced-gravity model with nonzero interfacial 
friction. This explains why for large values of the bottom friction coefficient, the dynamical regimes of a time-dependent two-layer model, described in the following section, closely resemble those of a reduced-gravity model with weak interfacial friction.

\subsection{Sensitivity Study vs Bottom Friction Coeffi- cient}

Case: $\alpha=0$

The behavior of the two-layer model was investigated with a set of various bottom friction parameters. Table 5.1 classifies the resulting state vs $\mathcal{A}_{2}$ for the case when the lower layer inflow velocity was chosen zero, so that the model was forced only through the upper layer inflow and outflow conditions. In all the cases, with an increase in the values of the bottom friction coefficient, the model showed the following progression of states:

- State $A$. The model has a unique dynamical regime when the flow consists of the large upper layer recirculations and smaller, eddy-driven lower layer recirculations. The total energy varies by only $3 \%$, which indicates weak variability associated with changes in the meridional position of the front and in the longitudinal extent of both the upper and the lower layer recirculating gyres. The example of the mean streamfunction field is given in Figure 5-5a,c. The detailed discussion of the state is given in section 5.4.

- State $B$. The upper layer flow structure consists of an eastward jet and two recirculating gyres. The flow in the lower layer is weak because of stronger bottom friction. The longitudinal size of the recirculations changes with time thus giving rise to strong low frequency variability. The latter displays itself in up to $16 \%$ deviations of the total energy from its mean value. The amplitude and the 
spatial structure of the eddy field are strongly time-dependent. The upper layer eddy field is characterized by the eastward and westward propagating meanders. Their interactions give birth to rings in the area east of the recirculation gyres in the upper layer. The example of a typical time mean streamfunction field is given in Figure 5-1, when $\mathcal{A}_{2}=5 \cdot 10^{-6} \mathrm{sec}^{-1}$.

- State $C$. The flow structure displays well-developed low frequency variability associated with two dynamical regimes: high energy state $B$ or low energy state $D$.

- State $D$. The upper layer flow is characterized by an eastward jet with large meridional excursions in the separation point. The corresponding eddy field is dominated by large-amplitude, westward propagating meanders and strong rings forming only on the western boundary. The recirculations are absent in instantaneous fields. The lower layer flow is characterized by two counterrecirculations that are very weak and are strongly frictional. The example of a typical time mean streamfunction field is given in Figure 5-1, when $\mathcal{A}_{2}=5 \cdot 10^{-5}$ $\sec ^{-1}$.

The next section presents the detailed analysis of a reference run with State $A$, when $\mathcal{A}_{2}=10^{-8} \mathrm{sec}^{-1}$. In states $B, C$ and $D$, the lower layer flow is strongly frictional, and hence these cases are of not much interest. In terms of the upper layer dynamics, States $B, C$ and $D$ of the two-layer model correspond to States $D, E, F$ of the reduced-gravity model. Figure 5-3 shows instantaneous streamfunction fields for state $C$, when $\mathcal{A}_{2}=7.5 \cdot 10^{-6} \mathrm{sec}^{-1}$. Clearly, the upper layer flow structure suffers significant changes with time. When the energy is high, the flow is characterized by large upper layer recirculations. As the recirculations decrease in size, the strength of the eddy field increases, giving rise to larger amplitude meanders and rings. When the energy is low, the flow is dominated by large amplitude, westward propagating meanders and strong rings forming on the western boundary. 


\begin{tabular}{|c|c|}
\hline \hline $\mathcal{A}_{2}^{\text {dim }}\left(\mathrm{sec}^{-1}\right)$ & STATE \\
\hline $1.0 \cdot 10^{-8}$ & $A$ \\
$2.0 \cdot 10^{-8}$ & $A$ \\
$3.0 \cdot 10^{-8}$ & $A$ \\
$5.0 \cdot 10^{-8}$ & $A$ \\
$1.0 \cdot 10^{-7}$ & $A$ \\
$8.0 \cdot 10^{-7}$ & $B$ \\
$1.0 \cdot 10^{-6}$ & $B$ \\
$3.0 \cdot 10^{-6}$ & $B$ \\
$5.0 \cdot 10^{-6}$ & $B$ \\
$7.5 \cdot 10^{-6}$ & $C$ \\
$1.0 \cdot 10^{-5}$ & $C$ \\
$3.0 \cdot 10^{-5}$ & $D$ \\
$5.0 \cdot 10^{-5}$ & $D$ \\
\hline
\end{tabular}

Table 5.1: Flow character for various dimensional bottom friction coefficients, when the lower layer inflow is zero, i.e. $\alpha=0$.

Case: $\alpha=0.1$

Table 5.2 classifies the flow regimes for the case when the lower layer inflow streamfunction was 10 times weaker than the corresponding upper layer inflow streamfunction, i.e. $\alpha=0.1$. Figure 5-4 shows time mean streamfunction fields for both layers. For small values of bottom friction parameter, the flow was characterized by large recirculations in the upper layer and small recirculations in the lower layer either pressed against the western boundary currents or located north and south of the eastward jet. There was not much low frequency variability in this case. For larger values of the bottom friction coefficient, the western boundary current separated earlier from the wall, giving rise to a wide, frictional eastward flowing jet. The upper layer flow with large recirculations showed strong low frequency variability associated with two dynamical regimes as described earlier. 


\begin{tabular}{|c|c|}
\hline \hline $\mathcal{A}_{2}^{\text {dim }}\left(\mathrm{sec}^{-1}\right)$ & STATE \\
\hline $1.0 \cdot 10^{-8}$ & $A$ \\
$1.0 \cdot 10^{-7}$ & $A$ \\
$5.0 \cdot 10^{-7}$ & $C$ \\
$1.0 \cdot 10^{-6}$ & $C$ \\
$5.0 \cdot 10^{-6}$ & $C$ \\
\hline
\end{tabular}

Table 5.2: Flow character for various dimensional bottom friction coefficients, when the amplitude of the lower layer inflow streamfunction is $10 \%$ of that of the upper layer inflow streamfunction, i.e. $\alpha=0.1$.

\section{Steady Solutions}

An attempt to find the antisymmetric steady solutions of the two-layer model by using a numerical code with enforced antisymmetry failed. The resulting statistically steady solutions were characterized by recirculating gyres that reached the sponge layer near the eastern wall. The total energy never attained a steady state due to the presence of a nonzero antisymmetric eddy field. In the original time-dependent model without artificial antisymmetry, the zonal extent of the recirculating gyres varied between 500 $\mathrm{km}$ and $2500 \mathrm{~km}$ depending on the value of the bottom friction coefficient.

The numerical experiments with the reduced-gravity, colliding jets model in Chapter 4 showed that biharmonic diffusion was not effective in dissipating energy, and therefore interfacial drag was required to reach a statistically steady solution. In the present two-layer model, the upper layer has only biharmonic diffusion, which may explain the absence of a steady antisymmetric solution with a reasonable zonal extent of the recirculating gyres. Therefore, the presence of bottom friction in the lower layer and the time-dependence of the upper layer potential vorticity field are required for the existence and maintenance of the observed statistically steady state. Perhaps, the model could have had steady antisymmetric solutions if a different frictional operator, such as lateral diffusion, were applied. 
It is unclear whether the model has other, asymmetric, steady states; this problem is beyond the scope of the present thesis. One may argue that, if they exist, the model might display multiple dynamical regimes. However, when both barotropic and baroclinic instabilities are present, the model dynamics becomes more complicated, and the steady states might not be useful in describing the behavior of system. State $A$ can be considered as a strange attractor of a quasi-geostrophic, turbulent, two-layer model with weak bottom friction. As we show in the next section, in this parameter regime, the model does not have multiple dynamical states, but instead it is characterized by only one dynamical regime with weak low frequency variability.

\subsection{Reference Run}

\subsubsection{Dimensional Parameters}

The reference run for the two layer model has the following dimensional parameters:

$$
\begin{gathered}
L=2400 \mathrm{~km}, \\
L_{x}=4667 \mathrm{~km}, \\
L_{d e f}=47.64 \mathrm{~km} \\
\mathcal{A}_{6}^{d i m}=8 \cdot 10^{10} \mathrm{~m}^{4} \mathrm{sec}^{-1} \\
\mathcal{A}_{2}^{d i m}=1 \cdot 10^{-8} \mathrm{sec}^{-1} \\
D_{1}=600 \mathrm{~m} \\
D_{2}=4000 \mathrm{~m} \\
U=1 \mathrm{~m} \mathrm{sec}^{-1} .
\end{gathered}
$$

The initialization procedure discussed in section 3.2 .9 was used to prescribe the boundary distributions (3.4) and (3.5) of the streamfunction field and its derivatives. 
(a)

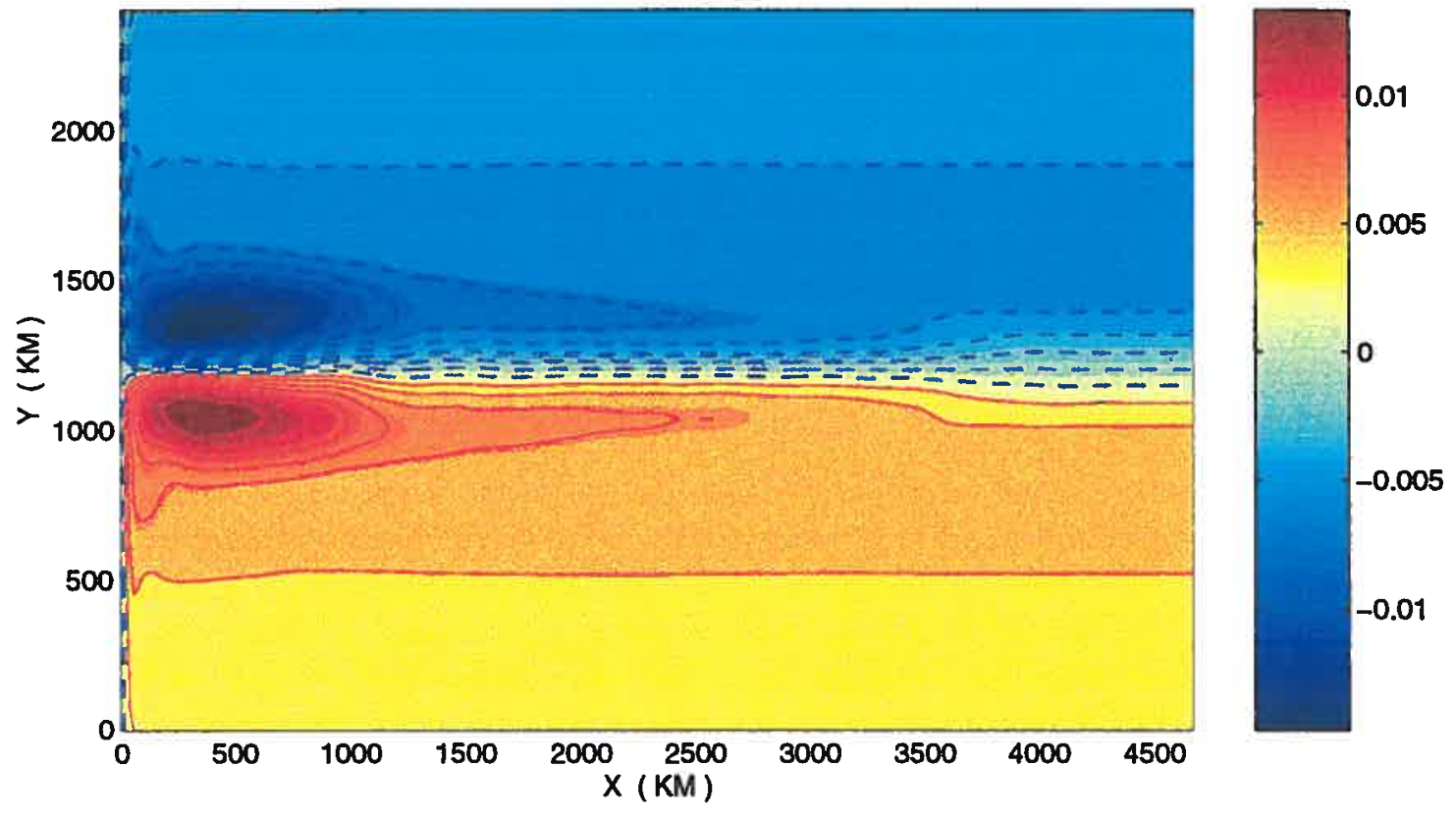

(b)

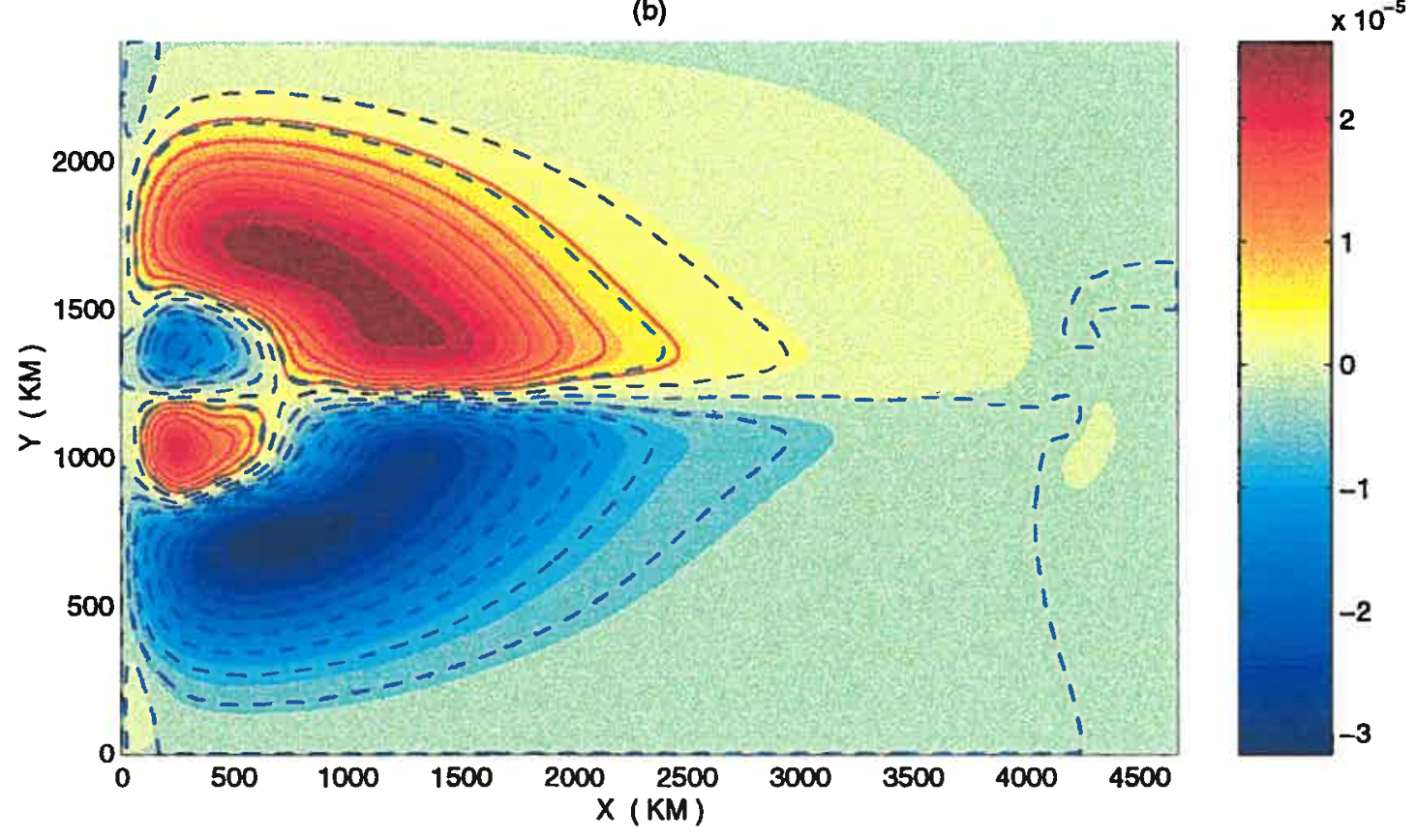

Figure 5-1: Upper layer (a) and lower layer (b) time mean streamfunction field, when $\alpha=0$ and $\mathcal{A}_{2}=5 \cdot 10^{-6} \mathrm{sec}^{-1}$. 
(a)

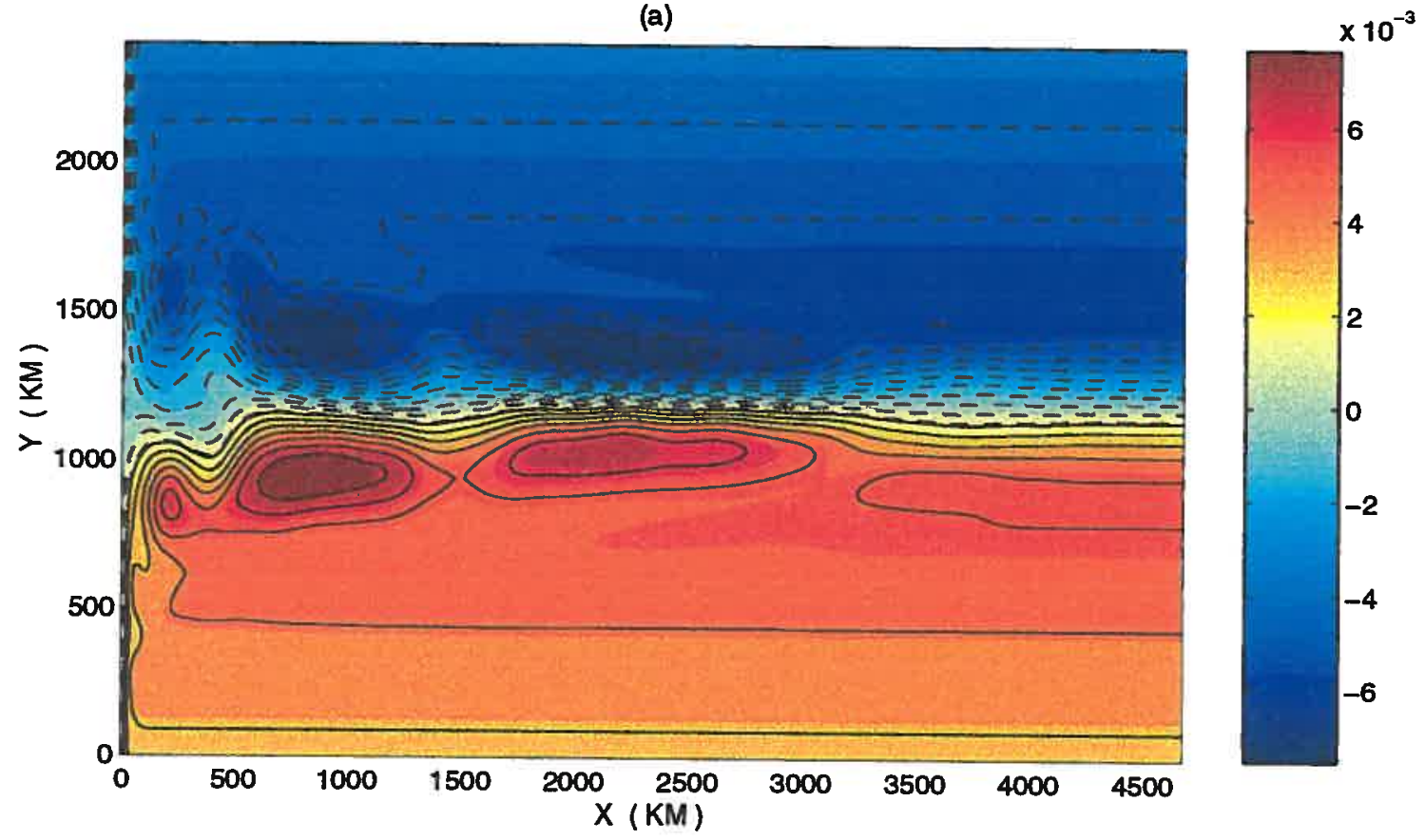

(b)

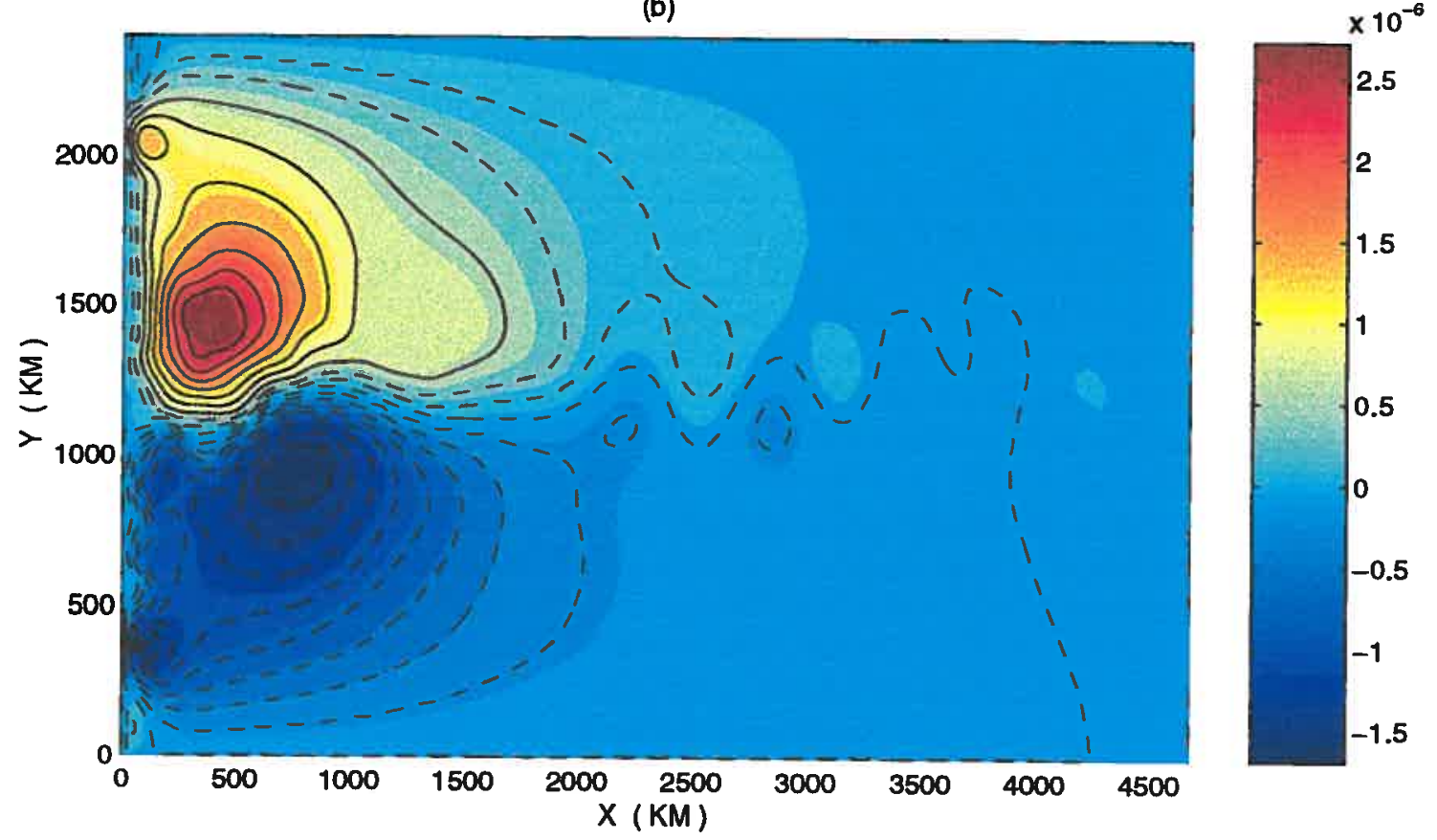

Figure 5-2: Upper layer (a) and lower layer (b) time mean streamfunction field, when $\alpha=0$ and $\mathcal{A}_{2}=5 \cdot 10^{-5} \mathrm{sec}^{-1}$. 
(a)

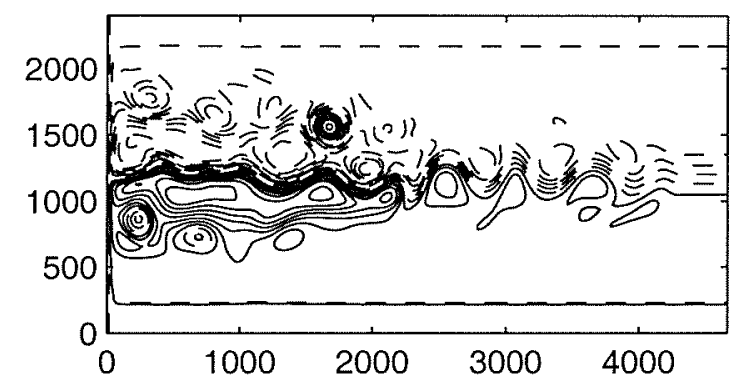

(c)

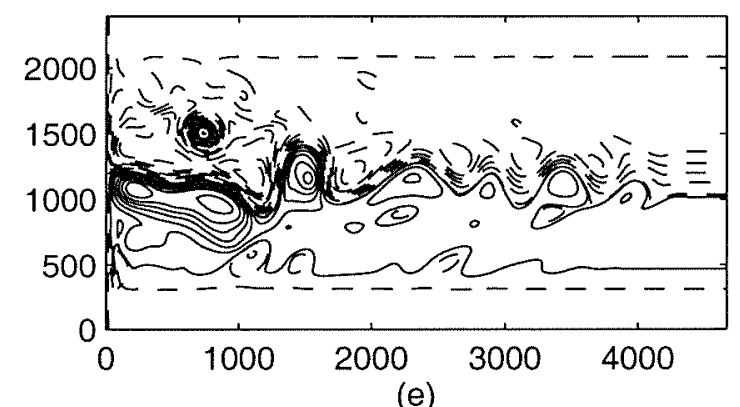

(e)

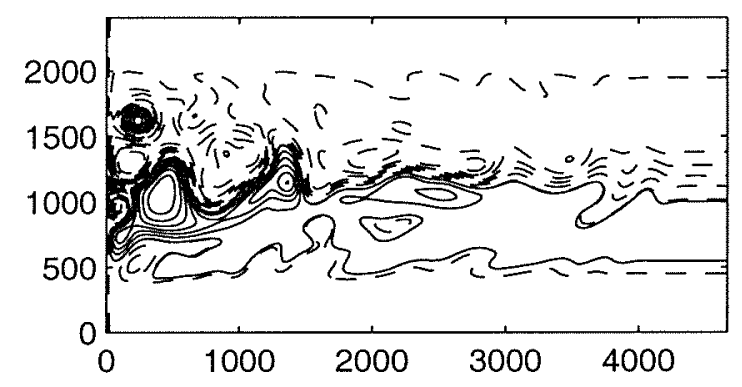

(b)

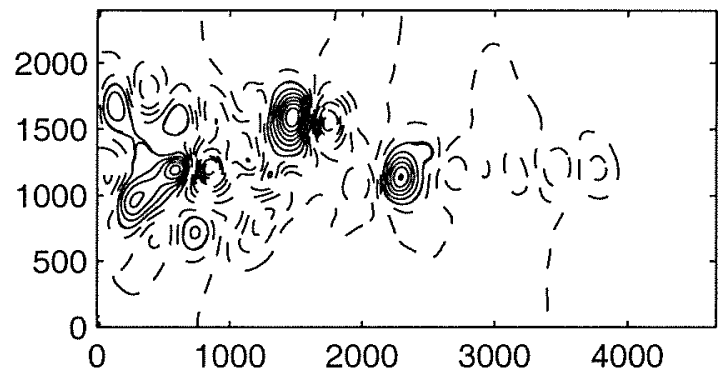

(d)
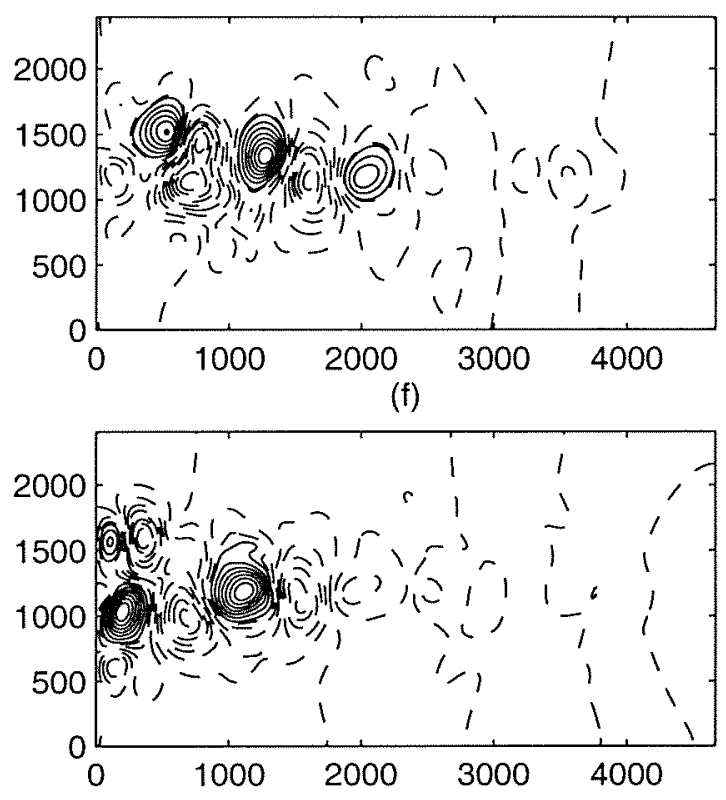

Figure 5-3: Instantaneous upper layer streamfunction (left column) and corresponding lower layer streamfunction (right column), when $\alpha=0$ and $\mathcal{A}_{2}=5 \cdot 10^{-5} \mathrm{sec}^{-1}$. The structure of the flow changes between a state with well developed recirculations in the upper layer and a weakly-meandering jet to a state with no upper layer recirculations and large amplitude, westward propagating meanders. 
(a)

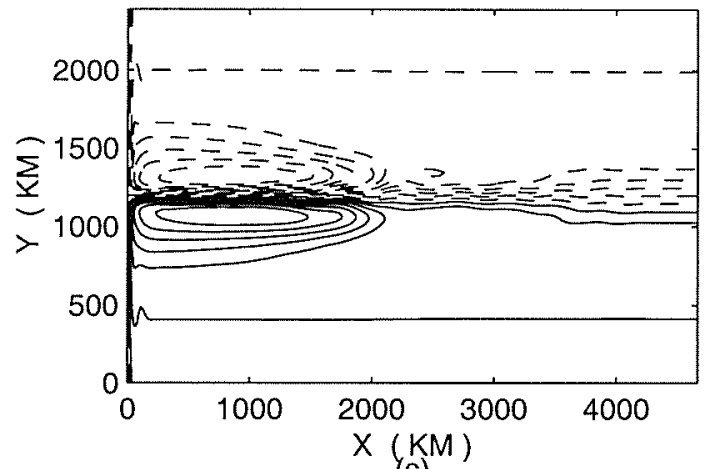

(c)
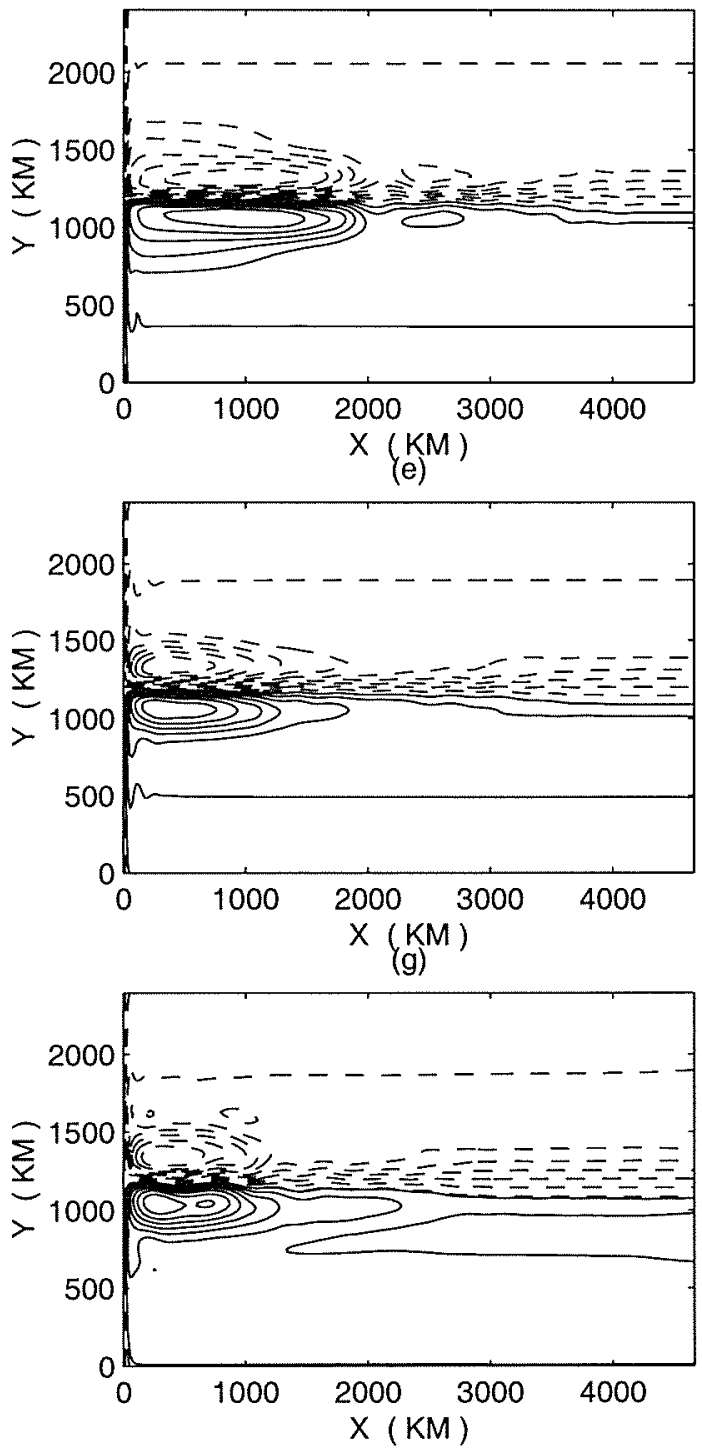

(b)

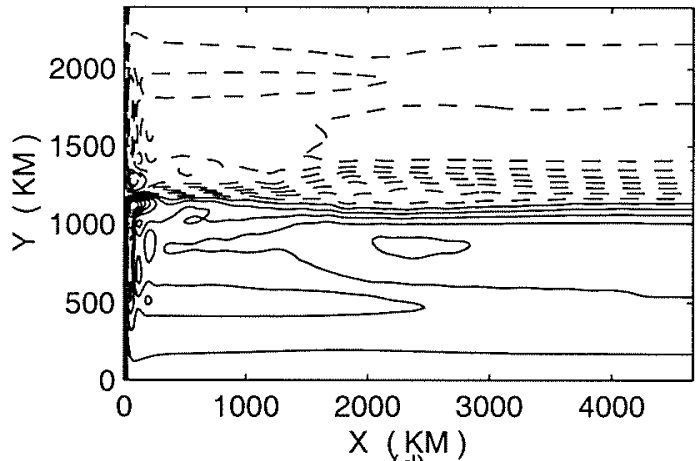

(d)

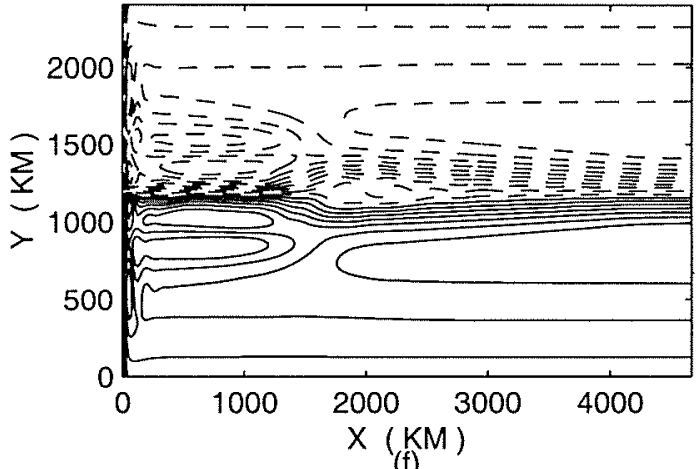

$(f)$

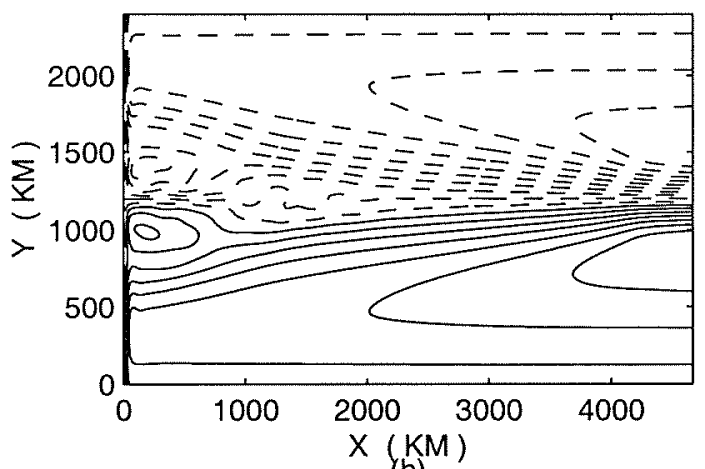

$($ h)

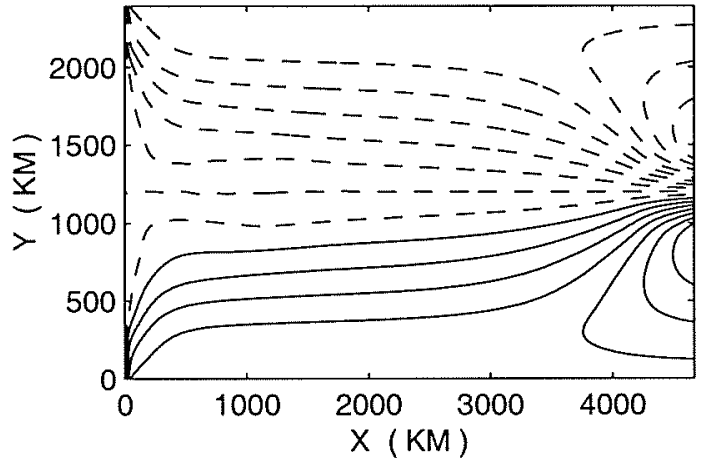

Figure 5-4: Upper layer (a,c,e,g) and lower layer (b,d,f,h) time mean streamfunction field, when $\mathcal{A}_{2}=10^{-8} \mathrm{sec}^{-1}, \mathcal{A}_{2}=10^{-7} \mathrm{sec}^{-1}, \mathcal{A}_{2}=5 \cdot 10^{-7} \mathrm{sec}^{-1}$, and $\mathcal{A}_{2}=5 \cdot 10^{-6}$ $\sec ^{-1}$ respectively. Lower layer inflow in nonzero: $\alpha=0.1$. 
The nondimensional amplitude of the inflow was chosen as $R_{1}=1$ for the upper layer and $R_{2}=0$ for the lower layer. Hence, the energy input into the model domain was only due to the upper layer inflow, and the main dissipation mechanism was provided by bottom friction. Since the interfacial drag was zero, the circulation in the lower layer was induced due to horizontal transfer of heat by the eddy fluxes.

The model was spun up for 30 years, until a statistically steady state was reached. An additional 424 years of integration allowed collection of the necessary statistics.

\subsubsection{Time Mean Flow}

As shown later, the model has one preferred energy state associated with a unique dynamical regime. Figure 5-5 shows the time mean fields of streamfunction and potential vorticity. The upper layer flow is characterized by two symmetric recirculating gyres, which are $2000 \mathrm{~km}$ long and $400 \mathrm{~km}$ wide. The time mean potential vorticity field reveals two regions of anomalous potential vorticity indicating waters brought by the western boundary currents from the north and south into the recirculation area. The upper layer recirculations are also well-defined in the instantaneous fields.

The lower layer flow has a more complicated spatial structure. There are two tight recirculations, $2000 \mathrm{~km}$ long and $200 \mathrm{~km}$ wide, in the middle of the domain. Two weak counter-rotating gyres are located to the north and south of the recirculations. The time-mean flows in the lower layer are driven by the eddy heat fluxes, and therefore they exist only in the time mean sense; the instantaneous fields are dominated by strong eddies. Outside the recirculation regions, the lower layer potential vorticity field is dominated by its planetary component, whereas in the area of the recirculations it is homogenized. Therefore, near the edges of the eastward jet, the time mean recirculations have the same sense of rotation in the upper and lower layers, whereas further south and north the time mean lower layer gyres rotate in the opposite direction to the upper layer flows, creating a region of strong baroclinic 
(a)

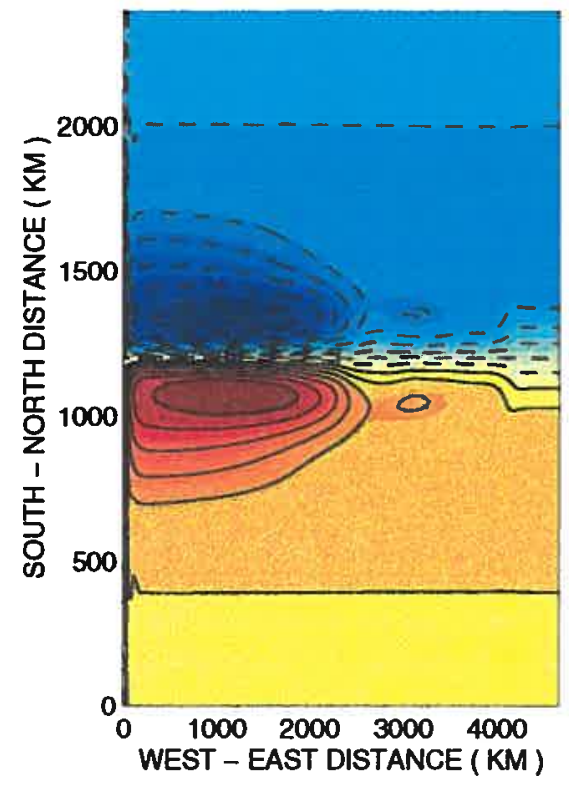

(b)
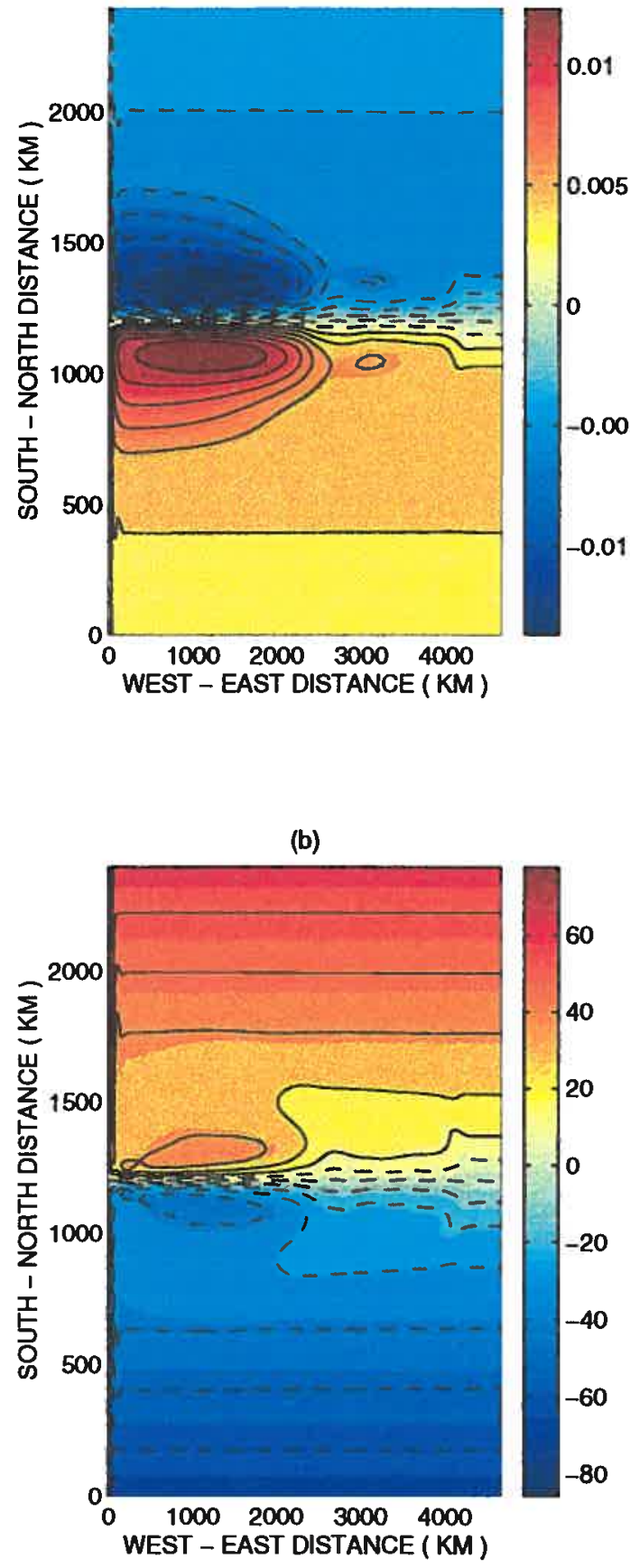

(c)

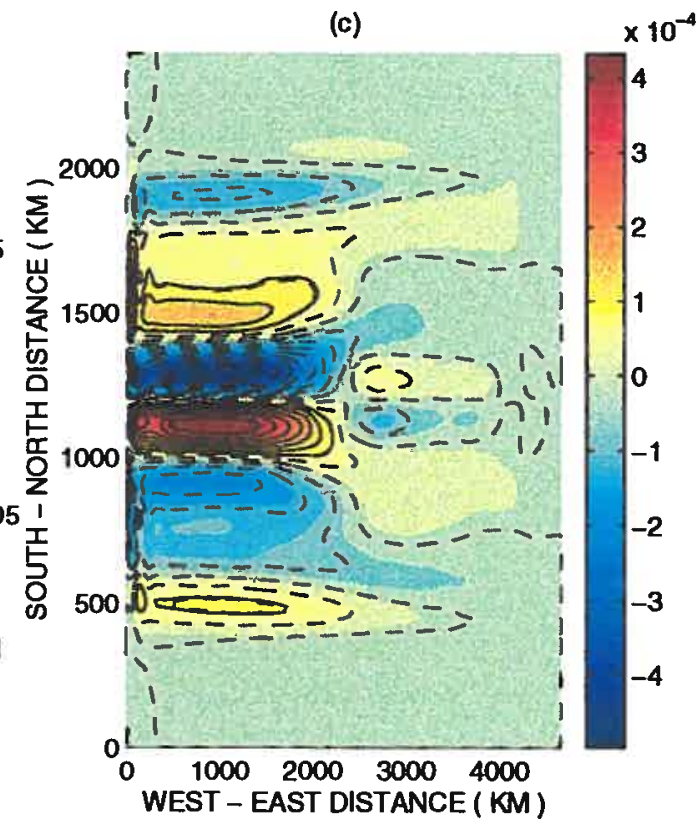

(d)

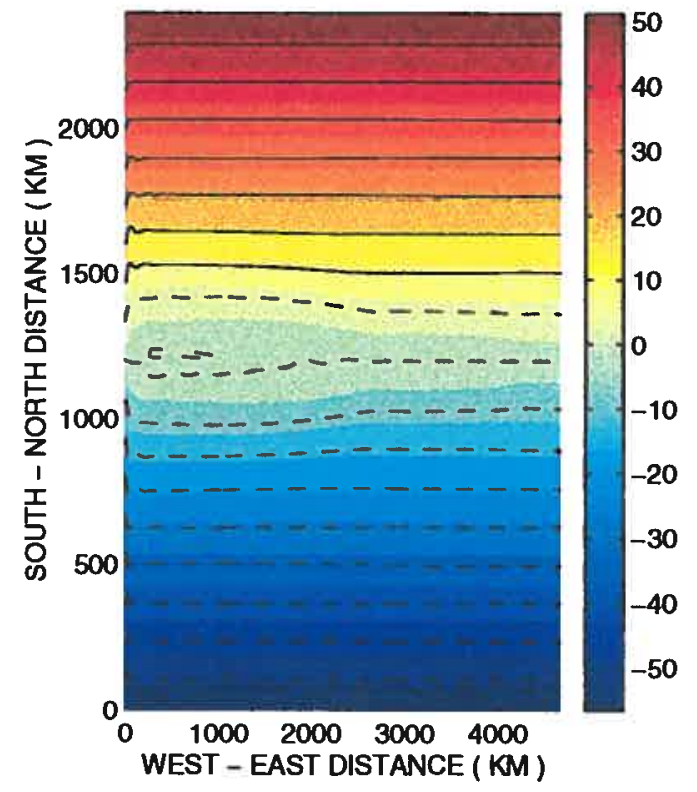

Figure 5-5: Contours of upper $(a, b)$ and lower $(c, d)$ layer streamfunction and potential vorticity fields. Parameters are defined in section 5.4.1. 
flow. The average total transport by both recirculations is about $40 \mathrm{~Sv}$, partitioned between $16 \mathrm{~Sv}$ in the lower layer and $24 \mathrm{~Sv}$ in the upper layer.

\subsubsection{Energetics}

The histograms (Figure 5-6) based on the time series of kinetic, potential and total energy indicate that the model has a unique energy state. To see whether the distributions are normal, a MATLAB QQPLOT routine was used to plot the quantities of a given time series versus the quantiles of the generated time series with normal distribution (Figure 5-7). The upper layer kinetic energy and potential energy time series are close to normal distribution. The tails of the lower layer kinetic energy time series do not fall within the error bars and therefore indicate significant deviation from normal distribution. The latter may be the result of the presence of strong vortices in the flow structure.

On average, total energy is partitioned between $87 \%$ potential, $9 \%$ upper layer kinetic and $4 \%$ lower layer kinetic energy. Therefore, the time series of total and potential energy are well correlated. Computed by using a MATLAB SPECTRUM routine with a hanning window of 20 and 100 years, the potential energy power density spectrum (Figure 5-8) is red with a distinct change in the slope at approximately 2 months. There is a distinct peak at approximately 1.4 years. Another peak is associated with a time scales larger than 10 years. Hence, similar to the reducedgravity model, the two-layer model exhibits low frequency variability. However, there is only one dynamical regime in the model, and it is associated with large recirculating gyres in the upper layer and with smaller eddy-driven gyres in the lower layer.

Peaks in the upper and lower layer kinetic energy spectrum (Figure 5-9) are located on the time scale of 1.4 years. The lower layer kinetic energy has a constant slope at time scales less than 1.4 years, whereas the upper layer kinetic energy spectrum changes the slope at 2 months. The latter must be related to the internal instability time scale. 

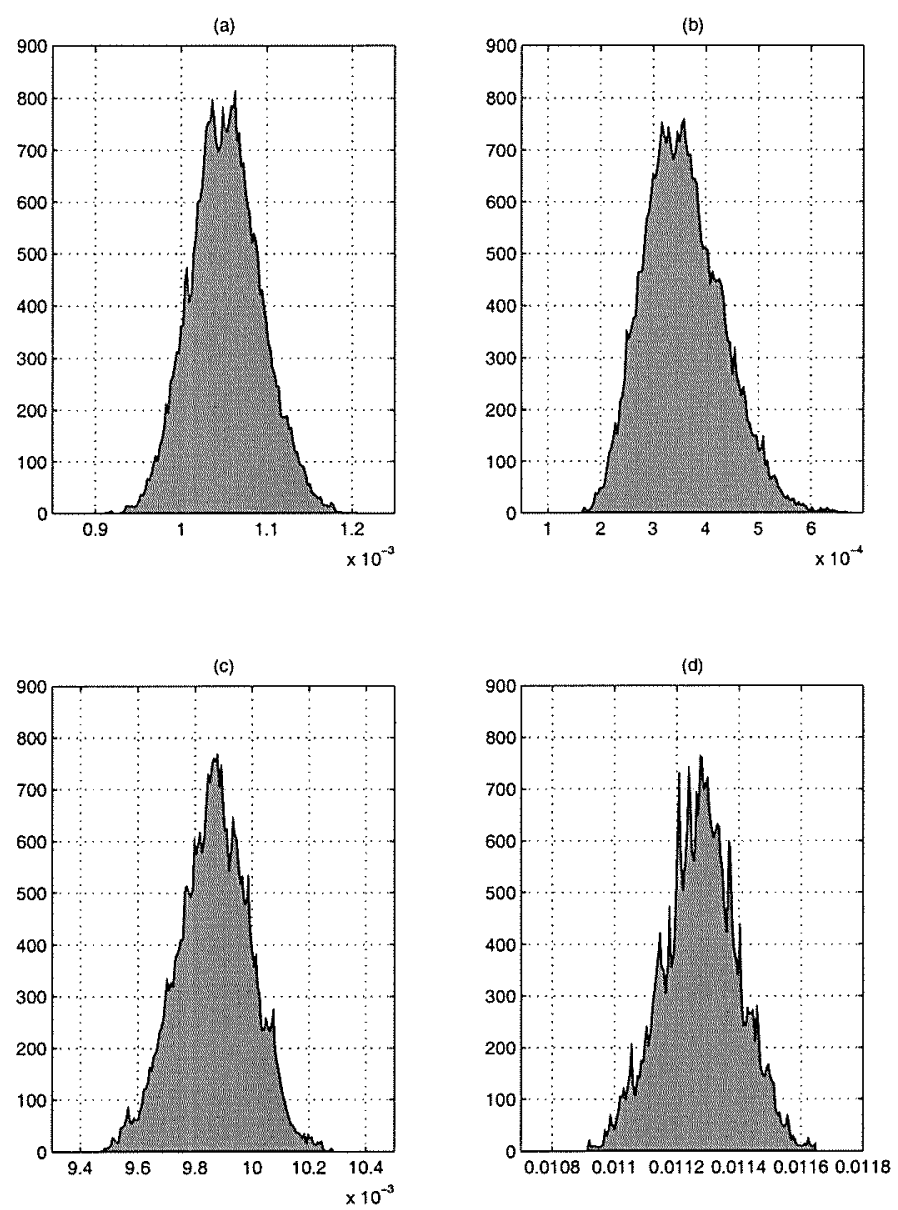

Figure 5-6: Histograms of upper layer kinetic (a), lower layer kinetic (b), potential (c) and total energy (d). Parameters are defined in section 5.4.1. 

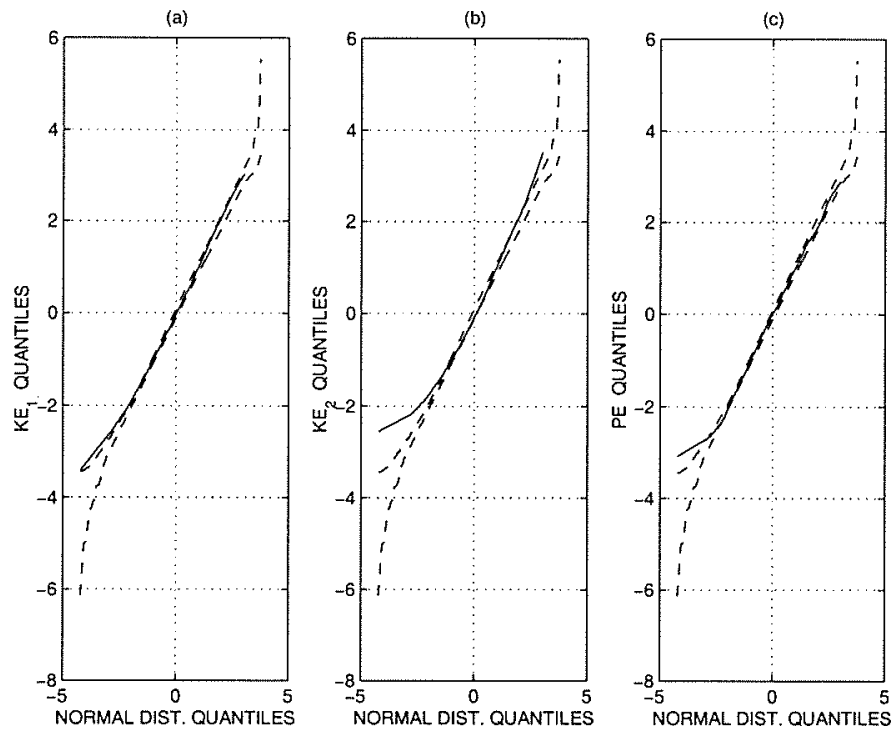

Figure 5-7: The quantile-quantile plot for various time series (solid lines) vs normal distribution. (a): upper layer kinetic energy, (b): lower layer kinetic energy, (c): potential energy. Dashed line denotes error bars. Parameters are defined in section 5.4.1.

Figure 5-10 summarizes the domain-integrated time mean energy conversion in the model. The energy input into the model domain is due to the upper layer inflows. The dissipation is provided through biharmonic diffusion and sponge absorption in both layers and through bottom friction. $57 \%$ of energy is dissipated through perturbations and $42 \%$ is dissipated by the mean flow. Total perturbation energy is almost equally partitioned between $K_{1}^{\prime}, K_{2}^{\prime}$ and $P^{\prime}$. The mean flow energy is partitioned between $91 \%$ of potential energy and $8 \%$ and $1 \%$ of upper and lower layer kinetic energy. In the domain-integrated sense, the barotropic conversion term is two orders of magnitude larger than the baroclinic conversion term. The bulk of barotropic conversion occurs in the upper layer, since the integral of $B T C$ in the lower layer is less than $1 \%$ of the total value. Berloff and McWilliams [4], in their two-layer wind-driven model with symmetric forcing and lateral diffusion, also observed strong barotropic dominance, when the flow stayed in the regime of strong jet penetration and large antisymmetric recirculations. In the wind-driven, double-gyre model of 

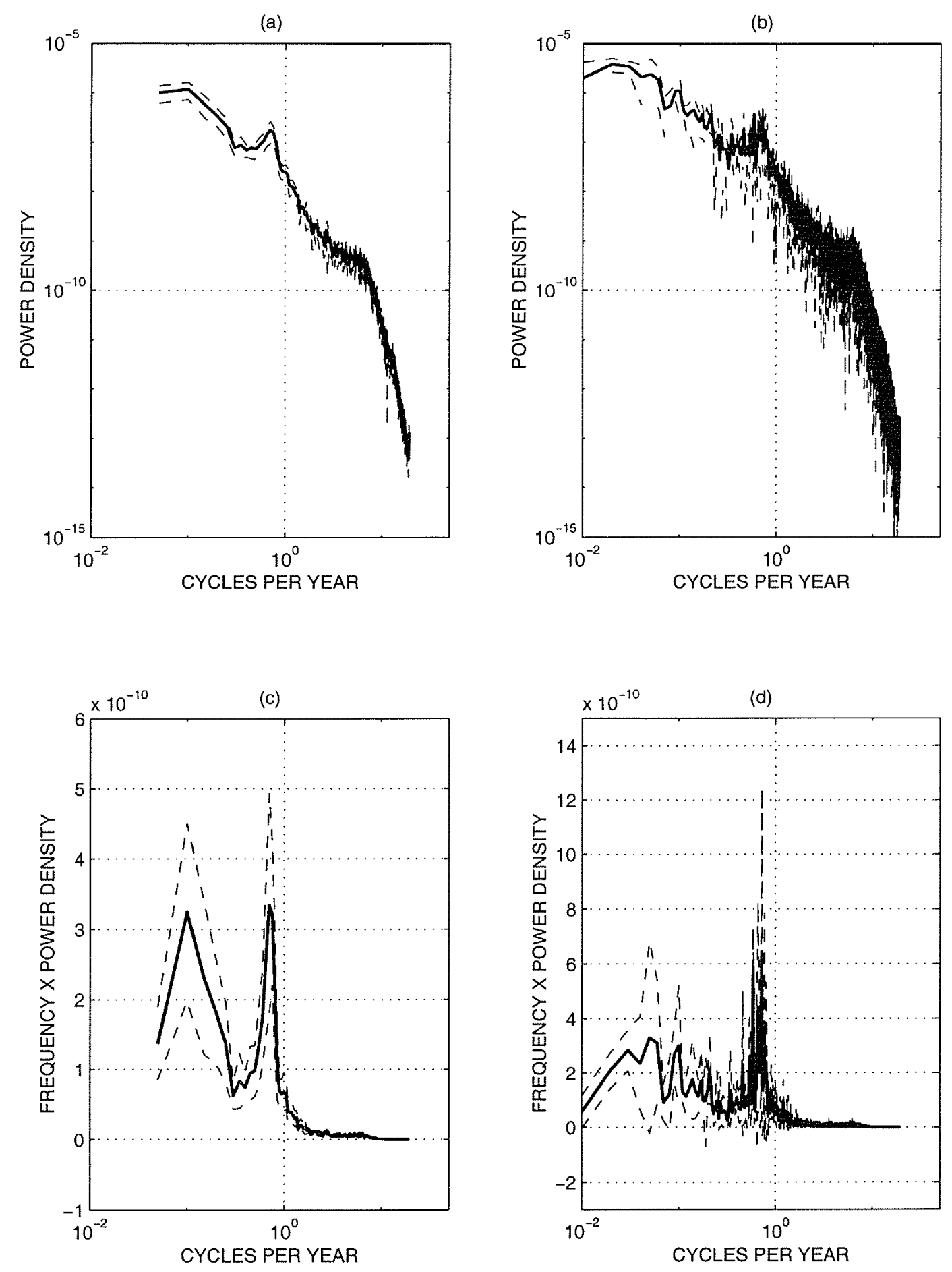

Figure 5-8: Potential energy power density spectrum (a) and frequency times potential power density spectrum (c) for window of 20 years. Dashed line denotes confidence interval at $95 \%$. (b) and (d): same for a 100-year long window. Parameters are defined in section 5.4.1. 

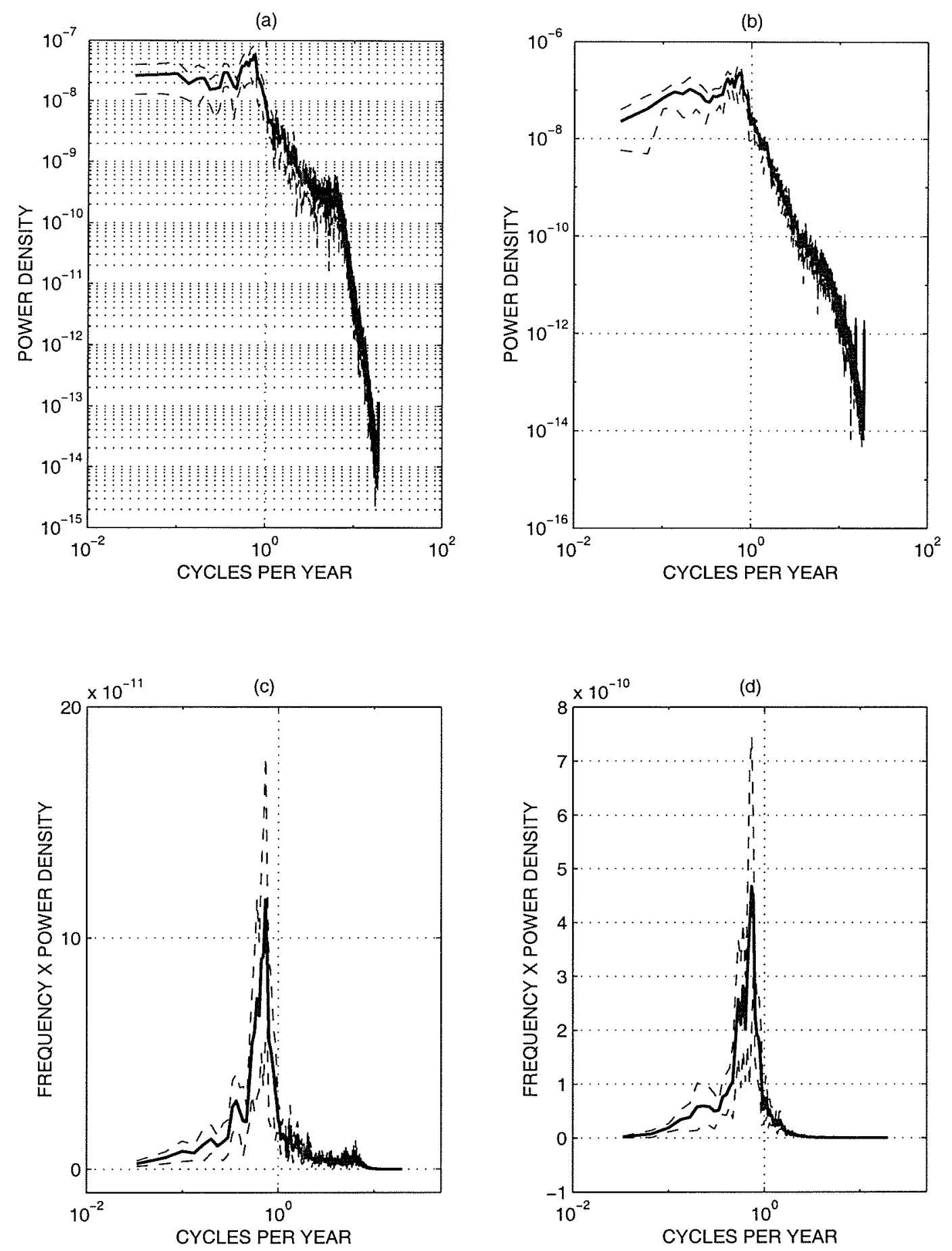

Figure 5-9: Upper layer kinetic energy power density spectrum (a) and frequency times upper layer kinetic power density spectrum (c) for window of 30 years. Dashed line denotes confidence interval at $95 \%$. (b) and (d): same the lower layer. Parameters are defined in section 5.4.1. 


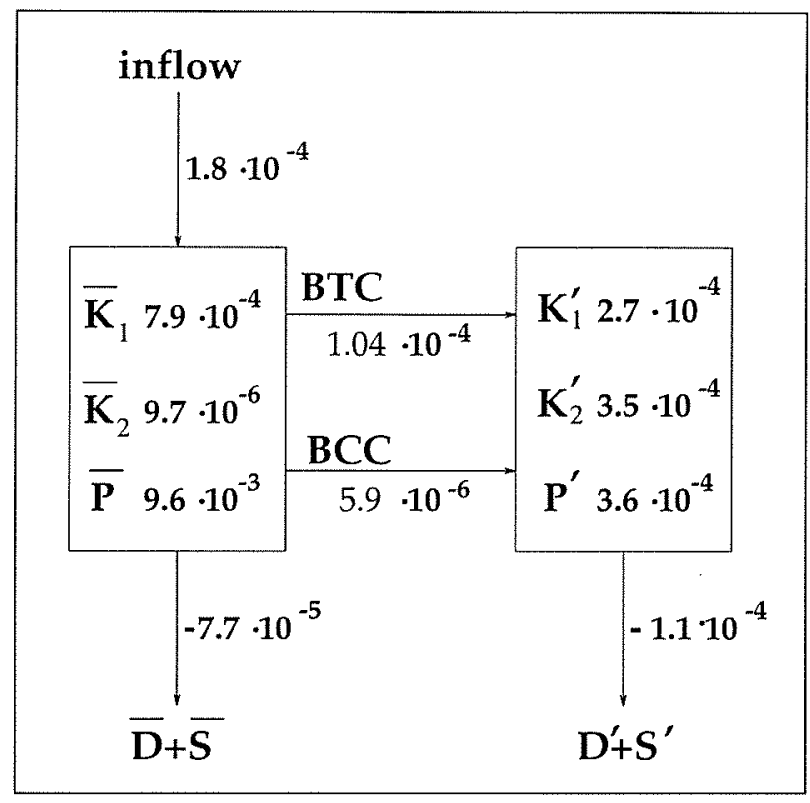

Figure 5-10: Energy conversion diagram. Parameters are defined in section 5.4.1

Holland [15], the baroclinic conversion term was also two orders of magnitude smaller than the barotropic conversion one in the experiment with small lateral diffusion.

\subsubsection{Time Mean Eddy Fields}

Figure 5-11 presents the energy density of the mean and eddy fields. The maxima in the upper layer mean kinetic energy are aligned with the eastward jet west of 2500 $\mathrm{km}$ and with the colliding western boundary currents. The lower layer mean kinetic energy has three maxima: one on the axis of the jet east of $2000 \mathrm{~km}$ and the other two in the region of the recirculating gyres. Two pools of maximum mean potential energy are associated with the recirculating gyres. Both perturbation potential energy and upper layer perturbation kinetic energy have maxima on the axis of the jet, which reflects the importance of meanders in the dynamics of the flow. The lower layer perturbation kinetic energy has two maxima in the western part of the recirculating gyres and along the western wall. 
a

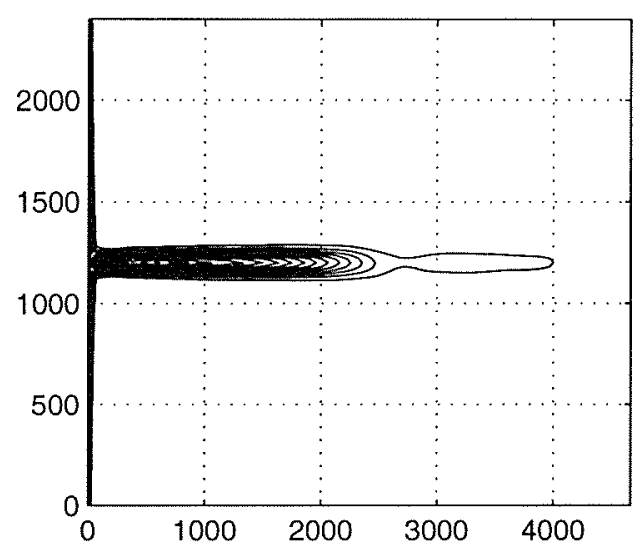

C

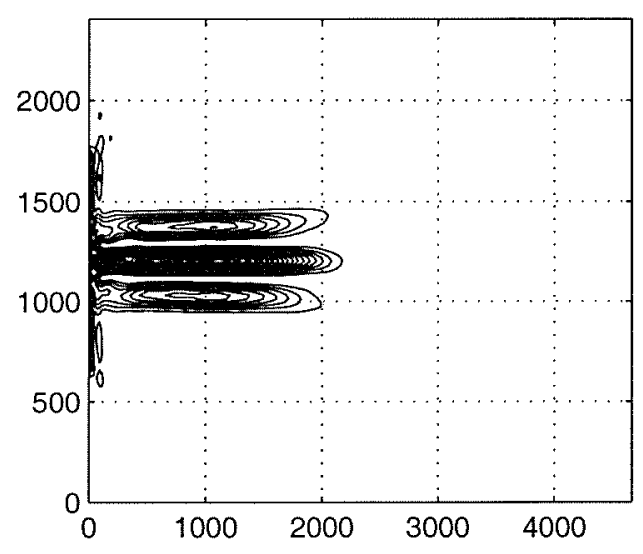

e

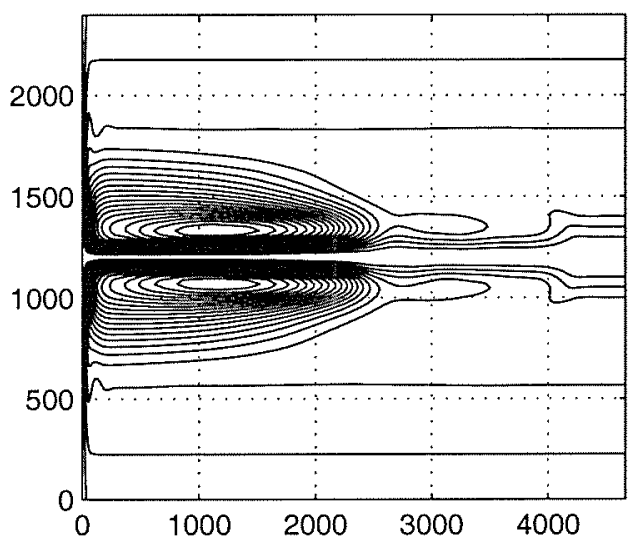

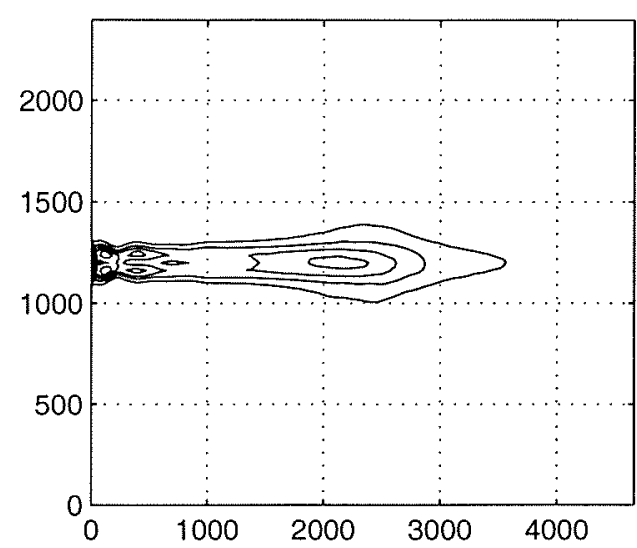

d
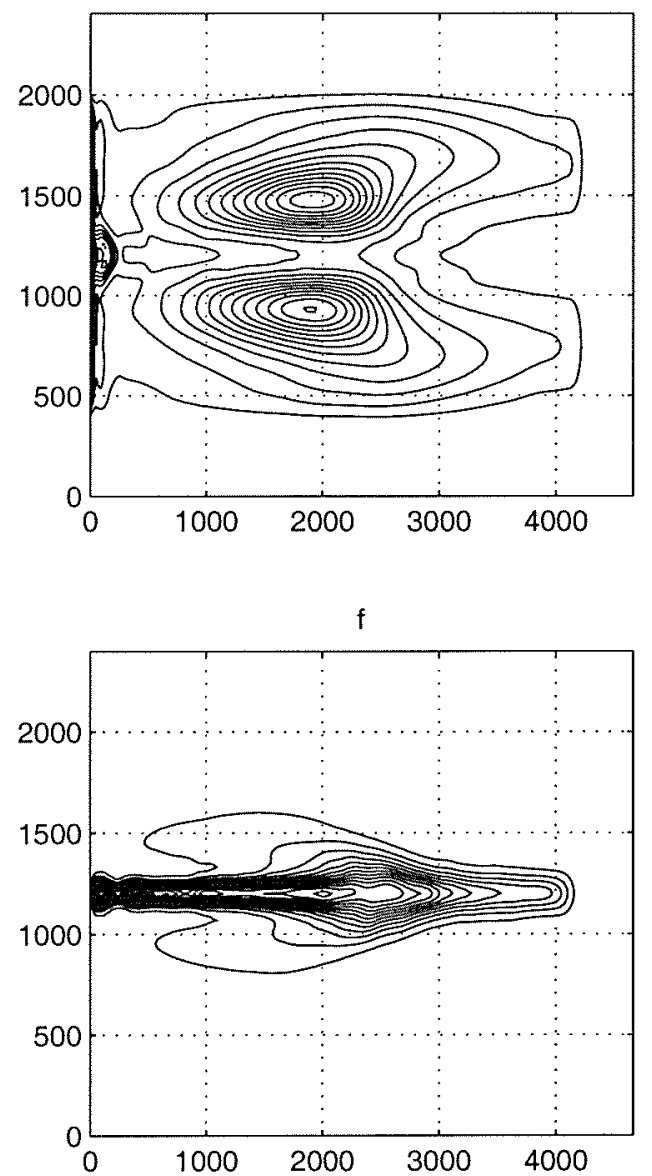

Figure 5-11: Energy densities: $\overline{K_{1}}(\mathrm{a}), \overline{K_{2}}(\mathrm{c}), \bar{P}(\mathrm{e}), K_{1}^{\prime}(\mathrm{b}), K_{2}^{\prime}(\mathrm{d}), P^{\prime}(\mathrm{f})$. 


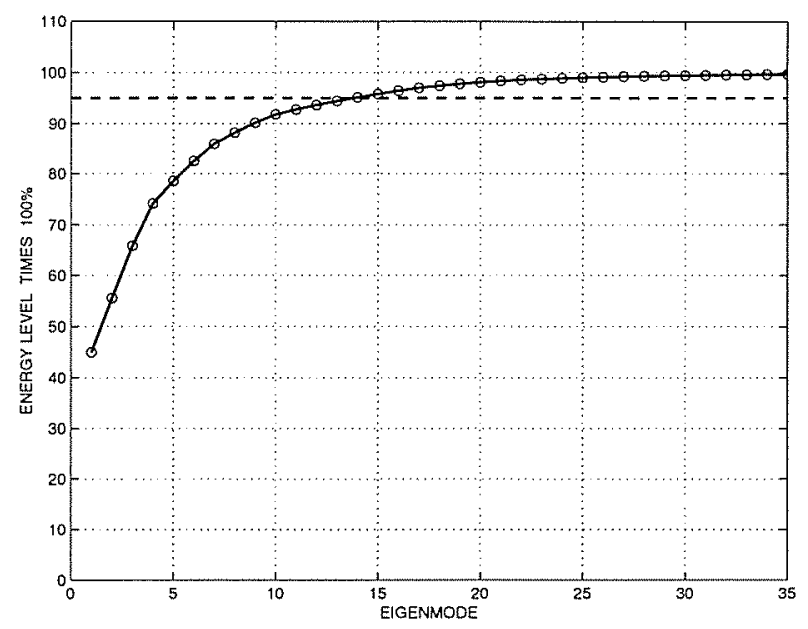

Figure 5-12: Energy level (in \%) for different eigenmodes resulted from an extended EOF analysis for a streamfunction fields, where time scales smaller than 10 years were filtered out. Dashed line denotes a $95 \%$ energy level.

\subsubsection{EOF Analysis of Low Frequency Variability}

To understand the nature of low frequency variability appearing as a wide peak on the time scales greater than 10 years in the potential energy spectra, an extended spatial Empirical Orthogonal Function (EOF) decomposition was performed for a sub-domain where the variability was maximum: $2917 \mathrm{~km} \times 1312 \mathrm{~km}$ centered near $y=0$. The streamfunction fields were first interpolated on to a regular grid of $32 \times 32$ points corresponding to the above sub-domain (the interpolation grid was different from the model grid). Second, a finite impulse response filter was applied to the time series to exclude time scales smaller than 10 years. Fourteen spatial EOFs were required to reach a 95\% energy level (Figure 5-12).

The first EOF is symmetric in both layers with a variance accounting for almost $45 \%$ of the total energy level. The corresponding spatial structure of the mode and its amplitude are shown in Figures 5-13a-c. The mode structure indicates variability associated with either northward or southward shifts in the position of the upper layer jet axis in the area between the recirculating gyres. The position of the separation point is fixed in the middle of the domain, which leads to the jet flowing either in the 
north-eastward or in the south-eastward directions after separation from the western boundary. In the lower layer, the mode tends to perturb the front near the separation point and to strengthen either the southern or northern recirculations. The spectrum of the time-dependent amplitude indicates a wide maximum on the time scales larger than 10 years with a slow decay towards smaller frequencies. Longer time series is required to isolate the variability on the scale close to 100 years.

The other $50 \%$ of the $95 \%$-energy level are partitioned between thirteen EOFs, with the second and the third one accounting for $10.6 \%$ and $10.3 \%$ respectively (Figures $5-13 \mathrm{~d}-\mathrm{i}$ ). The spatial structure of the second mode indicates variability in the area where the jet splits; it is associated with either southward and northward excursion of the front and with "wrapping" of the recirculations around each other. In the lower layer, the second mode tends to increase the longitudinal extent of one of the recirculations and decrease that of the other one. Also, it perturbs the front near the separation point.

When the amplitude of the third EOF-mode is positive, the upper layer recirculations tend to increase in their longitudinal extent, and the front has a weak north-west to south-east slope. In the lower layer, the symmetry of the flow breaks, since western part of the northern recirculation and the eastern part of the southern one tend to weaken. When the amplitude is negative, the upper layer recirculations shrink in zonal direction, and the slope of the front is from south-west to north-east. The lower layer recirculations appears weakened in the eastern part for the northern one and in the western part for the southern part.

A similar EOF decomposition based on a streamfunction time-series with variability larger than 20 years filtered out require ten EOFs for the $95 \%$ total energy level, with the most energetic mode having a $58 \%$ energy level. The spatial structure and the amplitude of the most important EOFs compare well with those based on a 10-year low-pass filter. 
(a)

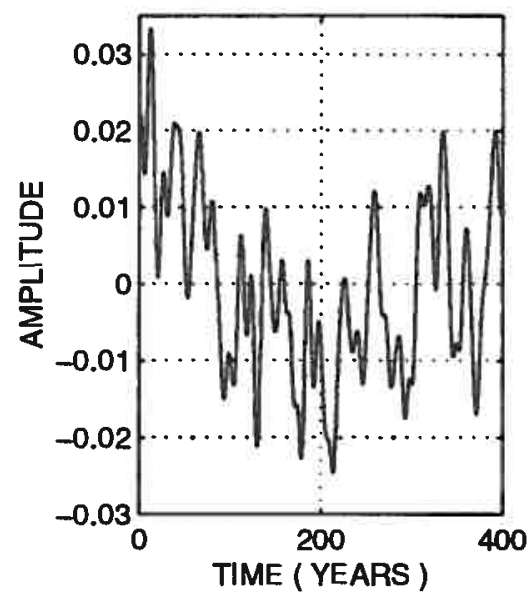

(d)

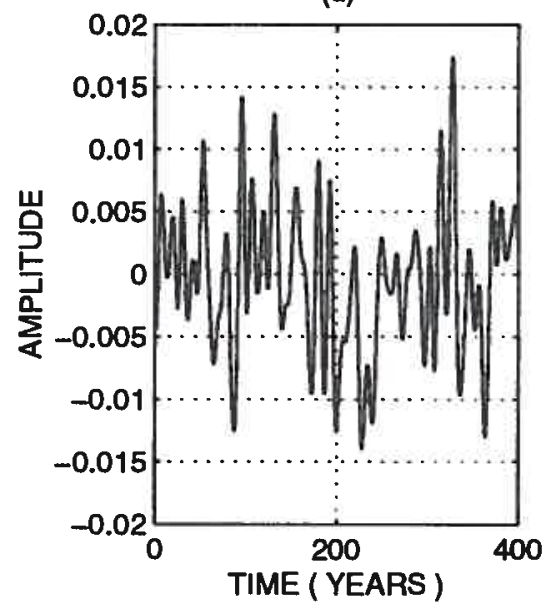

(g)

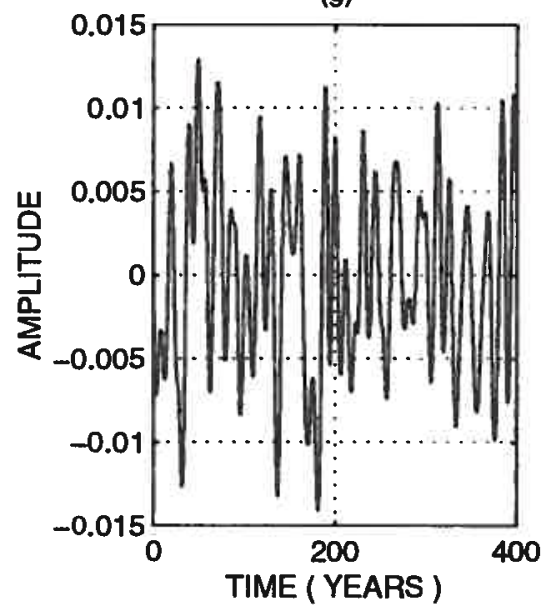

(b)

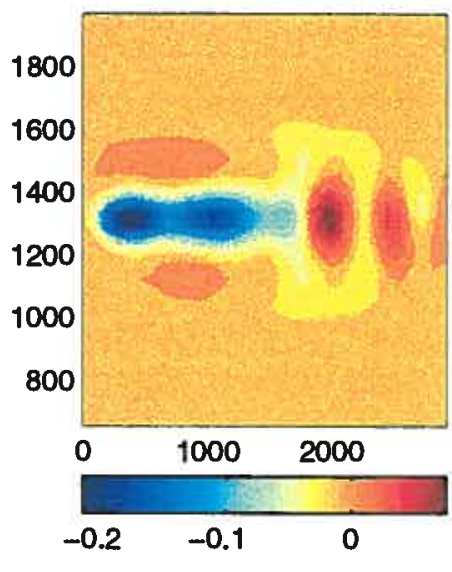

(e)

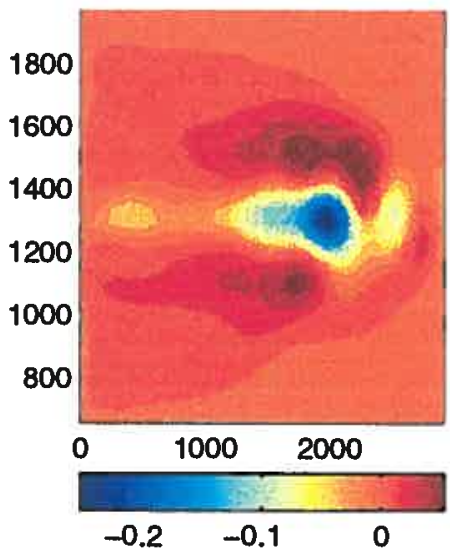

(h)

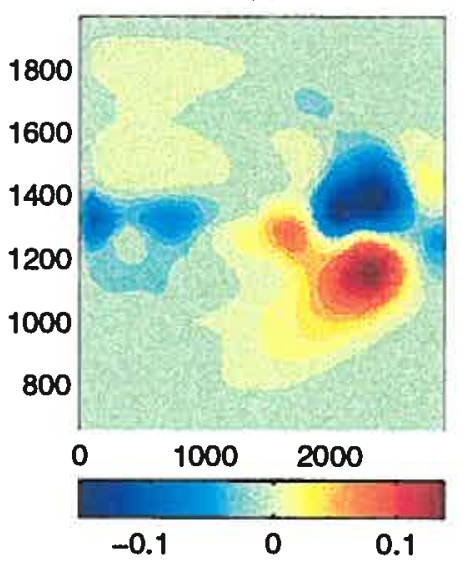

(c)

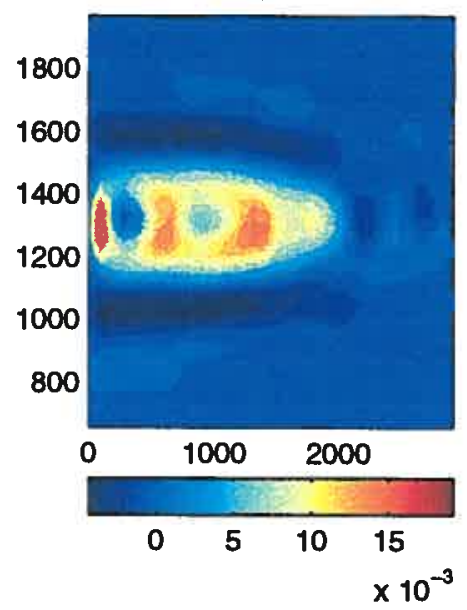

(f)

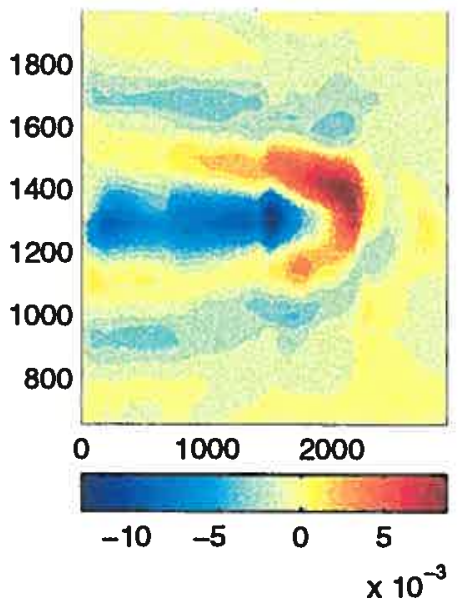

(i)

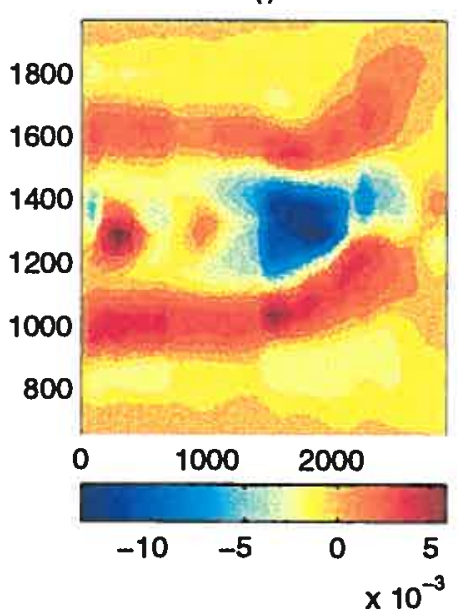

Figure 5-13: The time-dependent amplitude (a) and the upper (b) and the lower layer (c) spatial structures of the first EOF. Same for the second (d-f) and third (g-i) EOFs. The EOF analysis is based on the streamfunction field with variability larger than 10 years filtered out. 


\subsubsection{EOF Analysis of High Frequency Variability}

\section{Inter-annual Variability}

To study the inter-annual variability of the model, we performed an EOF analysis for the following two streamfunction time series. One was band-passed filtered for the variability between 1 and 10 years and the other one for the variability between 1 and 2 years. In both cases, the most important EOFs were remarkably similar, and the number of modes required for the $95 \%$ energy level was very close: 28 and 25 respectively. Therefore, most of the inter-annual variability is on the scales between 1 and 2 years, which is supported by the energy spectra plots of section 5.4.3. Thus, here we present the results of only the second EOF decomposition.

Figure 5-14 shows variance associated with 25 EOFs required for the $95 \%$ energy level with the first three functions having 17\%, 14\% and $11 \%$ energy levels respectively (for comparison: for the time series filtered between 1 and 10 years, the first three EOFs had 11\%, 10\% and $8 \%$ energy levels). The spectra of the timedependent amplitude and the corresponding spatial structure are presented in Figure 5-15. All modes are clearly associated with the meanders of approximately $1000 \mathrm{~km}$ length scale. The peak in amplitude spectra is around 1.3 years.

\section{Intra-annual Variability}

To study the inter-annual variability of the model, we performed an EOF analysis for the following two streamfunction time series. One was band-passed filtered for the variability between 1 and 12 months and the other one for the variability between 5 and 12 months. In both cases, the most important EOFs were remarkably similar. Here we present the results of only the second EOF decomposition.

Total of 35 modes required to reach the $95 \%$ energy level. The first three EOFs accounted for $24 \%, 18 \%$ and $9 \%$ of the total energy level. Their amplitude and spatial structures are presented in Figure 5-17. The modes are symmetric wave-like 


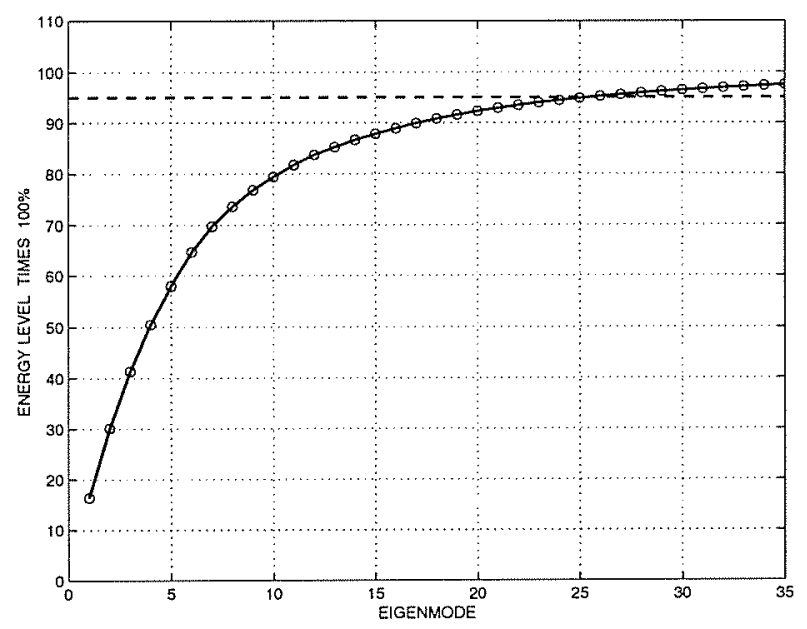

Figure 5-14: Energy level (in \%) for different eigenmodes resulted from an extended EOF analysis for a streamfunction with variability band-passed between 1 and 2 years. Dashed line denotes a $95 \%$ energy level.

structures sitting on the axis of the upper and the lower layer jet. The length scale of the modes, decaying west of $2000 \mathrm{~km}$, is approximately $1000 \mathrm{~km}$. The frequency multiplied by the power density spectrum of the time-dependent amplitude shows a peak at 8-9 months for all three modes. Therefore, the first three modes account for $51 \%$ of energy and are responsible for the variability on the time scales around 9 months. The other modes are each less energetic, but together they account for the remaining $44 \%$ of the total energy level.

\subsection{The Role of Baroclinic Instability in Low Fre- quency Variability}

\subsubsection{Method}

To study the role of baroclinic instability in low frequency variability, we employed a statistical method based on the approach of Davis and Emanuel [7]. Applied to the time series of the streamfunction fields, the method allows to define zones of baroclinic 

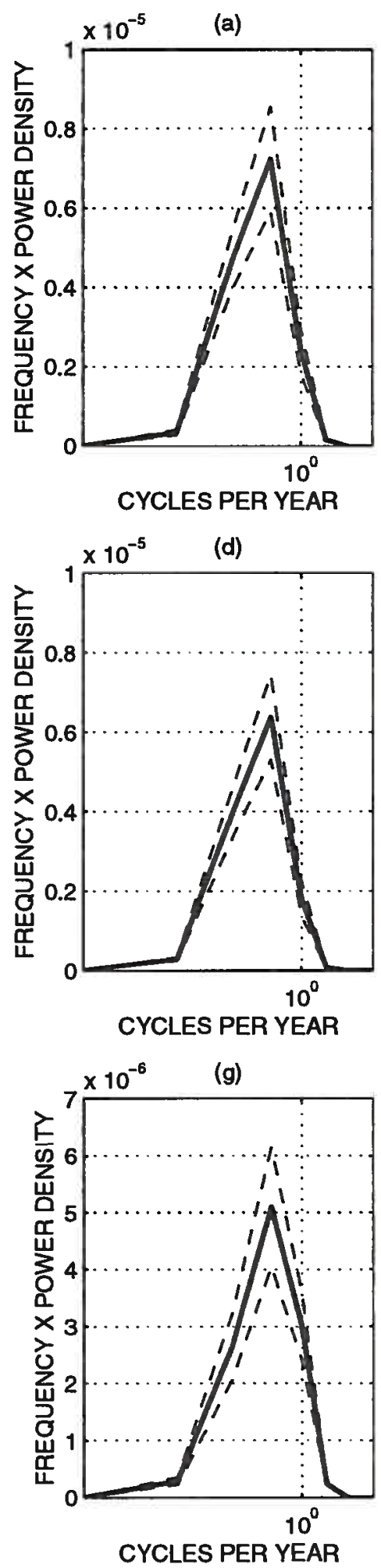

(b)

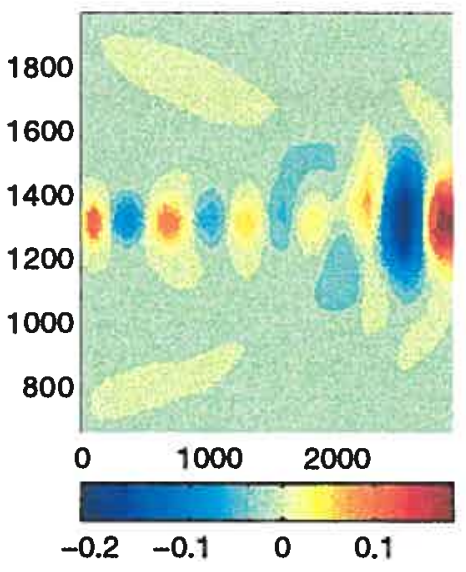

(e)

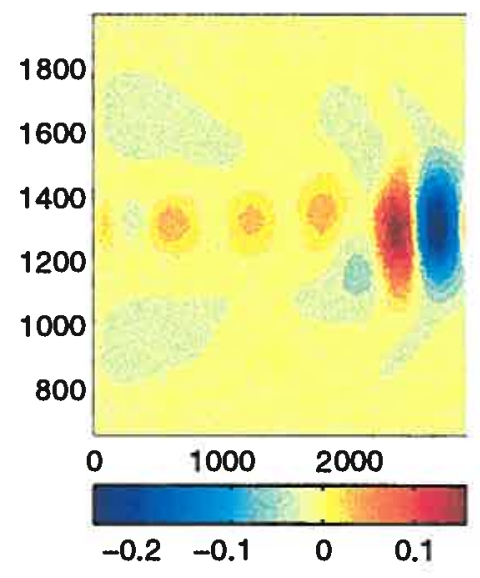

(h)

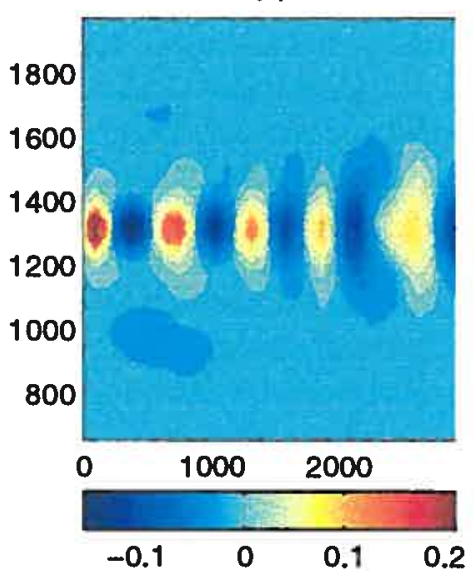

(c)

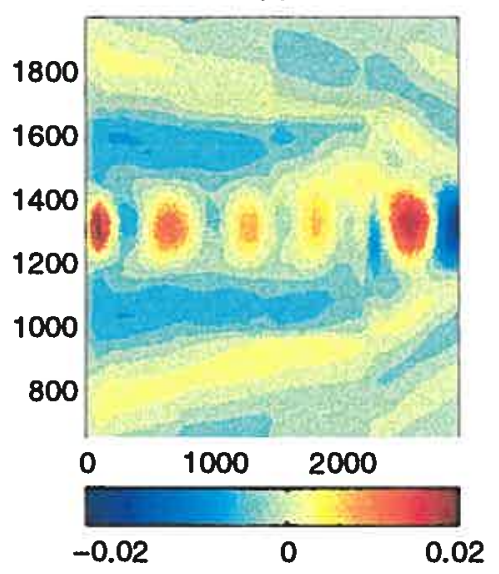

(f)

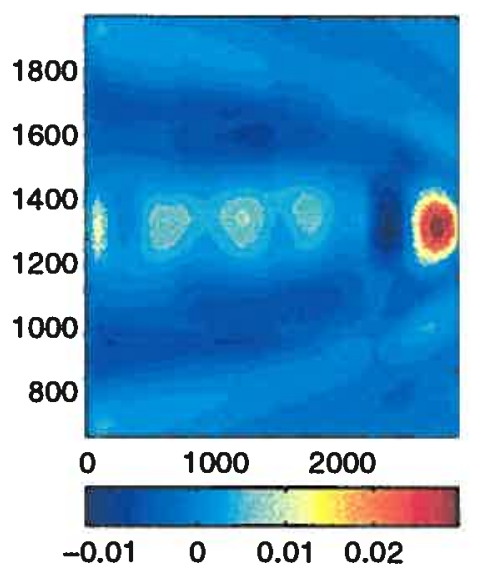

(i)

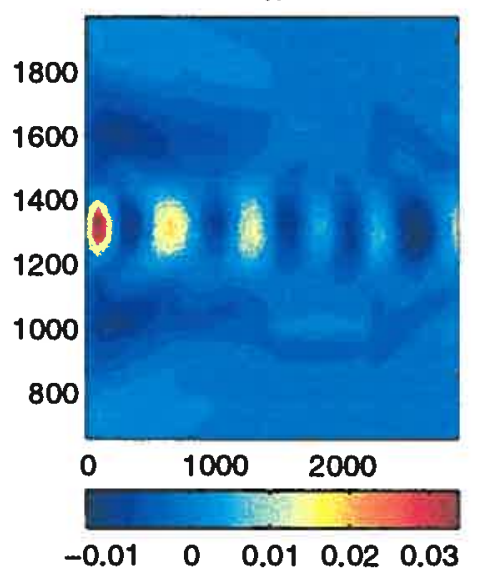

Figure 5-15: The time-dependent amplitude (a) and the upper (b) and the lower layer (c) spatial structures of the first EOF. Same for the second (d-f) and third (g-i) EOFs. The EOF analysis is based on the streamfunction field with variability between 1 and 2 years. 


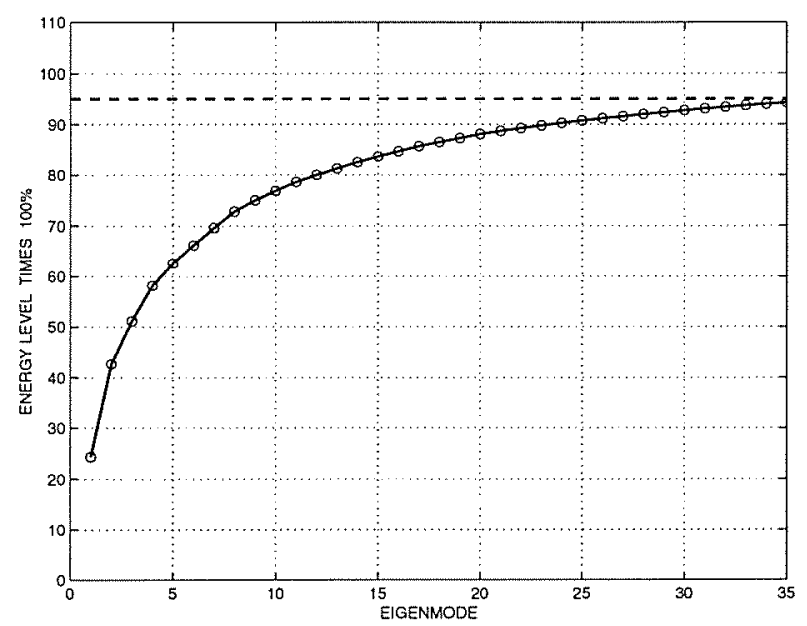

Figure 5-16: Energy level (in \%) for different eigenmodes resulted from an extended EOF analysis for a streamfunction with variability between 5 and 12 months. Dashed line denotes a $95 \%$ energy level.

growth in the time average sense. At each time step, the streamfunction and potential vorticity fields are decomposed into a time mean flow and perturbations:

$$
\psi_{i}(x, y, t)=\bar{\psi}_{i}(x, y)+\psi_{i}^{\prime}(x, y, t)
$$

and

$$
q_{i}(x, y, t)=\bar{q}_{i}(x, y)+q_{i}^{\prime}(x, y, t), i=1,2 .
$$

The perturbation streamfunction is further rewritten as a sum of a field associated with the upper layer potential vorticity anomaly and a field associated with the lower layer potential vorticity anomaly, i.e.

$$
\psi_{i}^{\prime}=\psi_{i}^{(1)}+\psi_{i}^{(2)}, i=1,2
$$

where $\psi_{i}^{(1)}$ is the solution of the problem:

$$
\left\{\begin{array}{l}
\left(\nabla^{2}-F_{1}\right) \psi_{1}^{(1)}+F_{1} \psi_{2}^{(1)}=q_{1}^{\prime}, \\
\left(\nabla^{2}-F_{2}\right) \psi_{2}^{(1)}+F_{2} \psi_{1}^{(1)}=0,
\end{array}\right.
$$

and $\psi_{i}^{(2)}$ is the solution of the problem:

$$
\left\{\begin{array}{l}
\left(\nabla^{2}-F_{1}\right) \psi_{1}^{(2)}+F_{1} \psi_{2}^{(2)}=0, \\
\left(\nabla^{2}-F_{2}\right) \psi_{2}^{(2)}+F_{2} \psi_{1}^{(2)}=q_{2}^{\prime} .
\end{array}\right.
$$



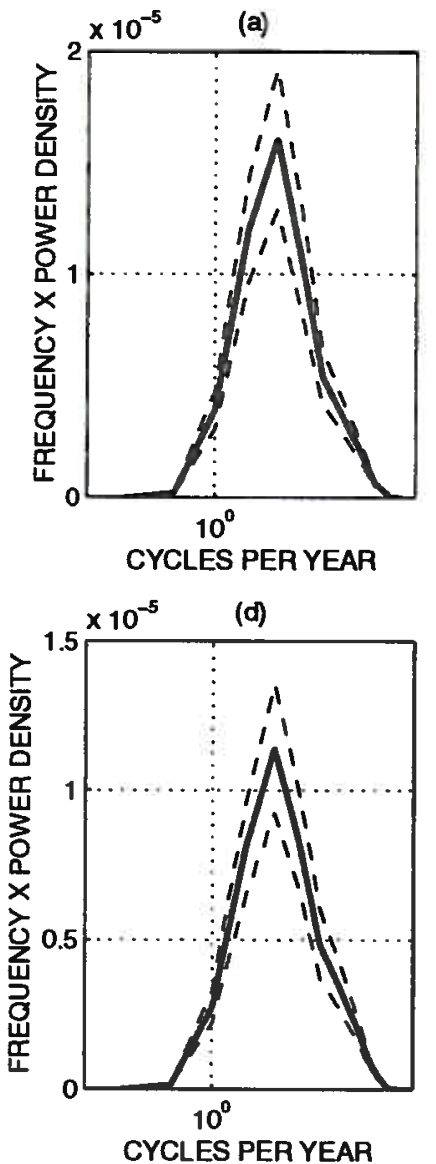

CYCLES PER YEAR

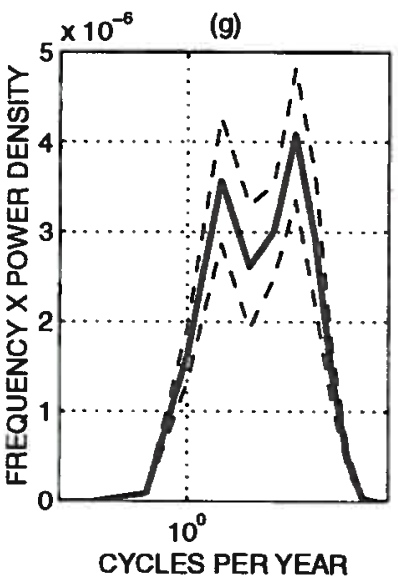

(b)

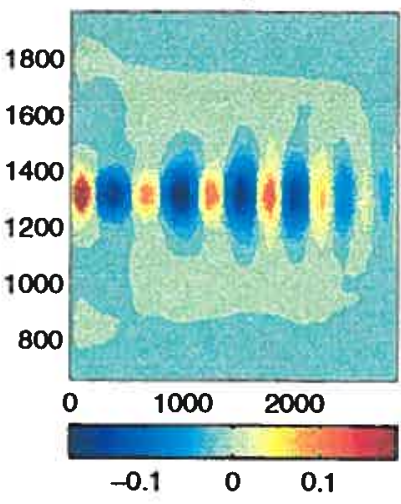

(e)

(c)

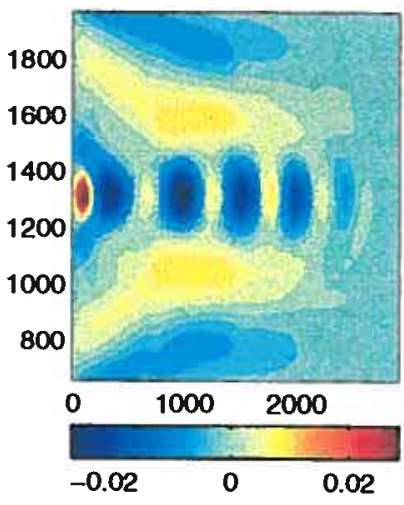

(f)

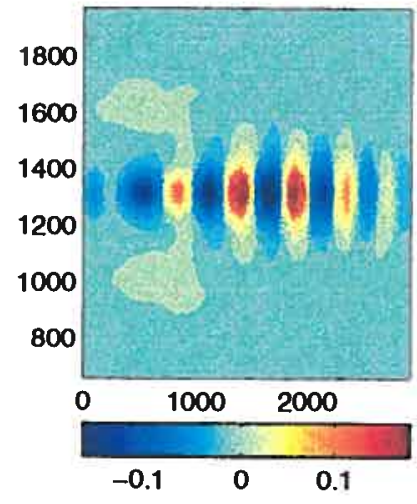

(h)
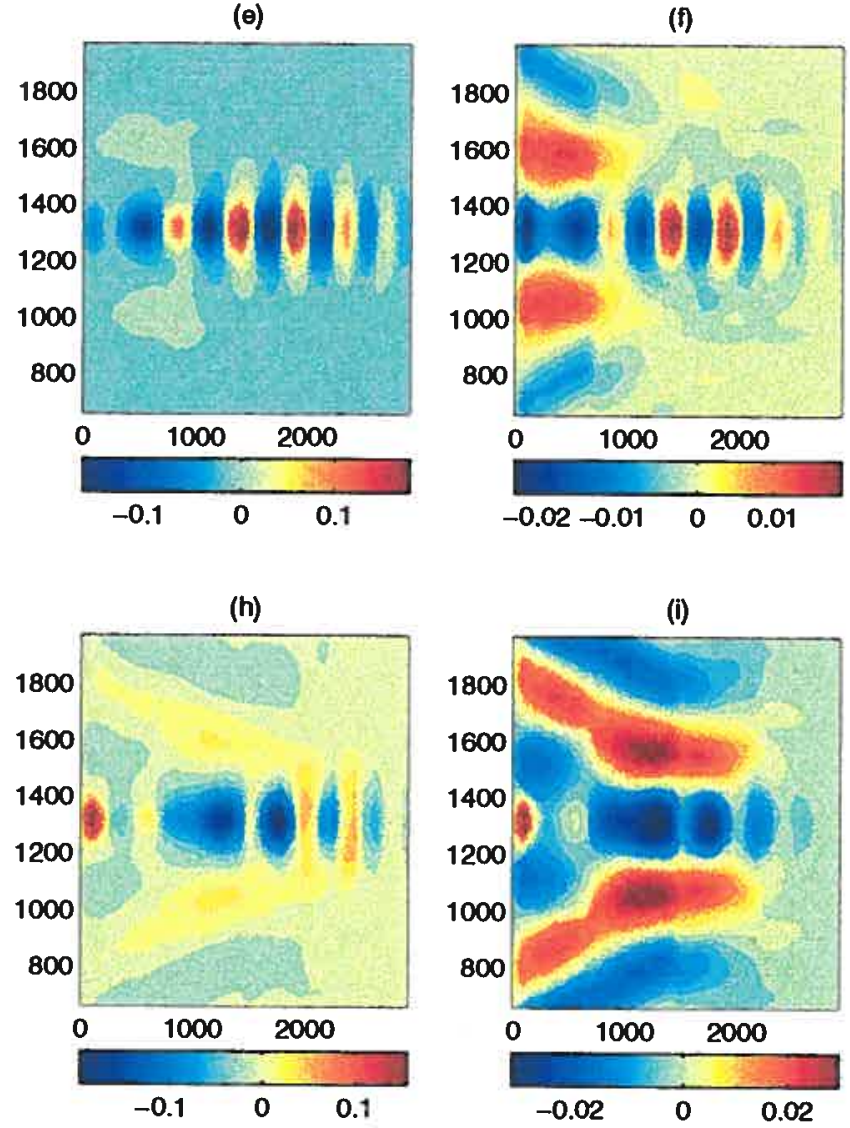

Figure 5-17: The frequency multiplied by the power density spectrum of the square of the time-dependent amplitude (a) and the contours of the upper (b) and the lower layer spatial structures (c) of the first EOF. Same for the second (d-f) and third (g-i) EOFs. The EOF analysis is based on the streamfunction time series with variability between 5 and 12 months. The spatial structure shown for the sub-domain, units are in $\mathrm{km}$. 
The linearized perturbation enstrophy equations then can be rewritten as:

$$
\begin{aligned}
& \frac{\partial}{\partial t} \frac{q_{1}^{\prime 2}}{2}+q_{1}^{\prime} \overline{\mathbf{u}}_{1} \cdot \nabla q_{1}^{\prime}+q_{1}^{\prime} \mathbf{u}_{1}^{(1)} \cdot \nabla \bar{q}_{1}+q_{1}^{\prime} \mathbf{u}_{1}^{(2)} \cdot \nabla \bar{q}_{1}=q_{1}^{\prime} D_{1}^{\prime}, \\
& \frac{\partial}{\partial t} \frac{q_{2}^{\prime 2}}{2}+q_{2}^{\prime} \overline{\mathbf{u}}_{2} \cdot \nabla q_{2}^{\prime}+q_{2}^{\prime} \mathbf{u}_{2}^{(1)} \cdot \nabla \bar{q}_{2}+q_{2}^{\prime} \mathbf{u}_{2}^{(2)} \cdot \nabla \bar{q}_{2}=q_{2}^{\prime} D_{2}^{\prime},
\end{aligned}
$$

where $\mathbf{u}_{i}^{(j)}=\left(-\frac{\partial \psi_{j}^{(j)}}{\partial y}, \frac{\partial \psi_{j}^{(j)}}{\partial x}\right)^{T}$ is a geostrophic velocity field. Zones of baroclinic growth are defined by $(x, y)$ where both of the following constraints are satisfied:

$$
\left\{\begin{array}{c}
\left\langle q_{1}^{\prime} \mathbf{u}_{1}^{(2)} \cdot \nabla \bar{q}_{1}\right\rangle<0, \\
\left\langle q_{2}^{\prime} \mathbf{u}_{2}^{(1)} \cdot \nabla \bar{q}_{2}\right\rangle<0,
\end{array}\right.
$$

where $\langle\cdot\rangle$ denotes time average. These inequalities can be interpreted as conditions for an increase in perturbation enstrophy due to a down-gradient eddy potential vorticity flux produced by the potential vorticity anomaly in the other layer.

\subsubsection{Applications}

The above method was applied for two different values of the bottom friction coefficient: $A_{2}=10^{-8} \mathrm{sec}^{-1}$ and $A_{2}=3 \cdot 10^{-6} \mathrm{sec}^{-1}$. Figure 5-18a shows that in the first case, there are two regions of baroclinic growth located in the area of westward recirculations accompanied by other two small zones north and south of where the jet splits. As was shown in the previous sections, for a given value of bottom friction coefficient, the total energy varies by less than $3 \%$ from its mean value, and the low frequency variability, although present, is weak.

For $A_{2}=3 \cdot 10^{-6} \mathrm{sec}^{-1}$, small zones of baroclinic growth are located on the most western and eastern edges of the recirculations, where the flow becomes predominantly meridional. The total energy level varies by $16 \%$, and the low frequency variability is strong.

Therefore, for small values of the bottom friction parameter, the presence of baroclinic instability in the recirculation area reduces the strength of low frequency 
variability. In these experiments, multiple dynamical regimes do not occur when baroclinic instability is present in the recirculation region.

\subsection{Summary and Discussion}

In Chapter 5, we described the results of the numerical experiments with a two-layer, colliding jets model which showed strong dependence of the flow structure on the bottom friction parameter. For small bottom friction, which implied a physically relevant lower layer flow, the model stayed in a unique dynamical regime associated with the well-developed recirculations in both layers. To understand the nature of low and high frequency variability observed in the model, an extended spatial EOF analysis was performed for filtered streamfunction fields. The low frequency spatial EOFs were found to explain decadal and longer variability in the position of the eastward jet axis, as well as weak wrapping and zonal pulsations of the recirculation gyres. For quasi-annual and mesoscale variability, the EOF analysis showed the importance of wave-like structures sitting on the axis of the jet between the recirculating gyres with the length scale of $1000 \mathrm{~km}$ and the time scale of 8 to 9 months.

For large values of the bottom friction coefficient, the lower layer flow became

strongly frictional, and the recirculations disappeared. However, the dynamics of the upper layer flow became more complicated displaying both multiple dynamical regimes and low frequency variability which closely resembled those of the reducedgravity model with weak interfacial friction. Such similarity between the reducedgravity and the two-layer models was possible because of the forcing provided by the lower layer flow on the upper layer flow and associated with strong bottom friction.

The results of the two-layer calculations showed that for physically feasible values of the bottom friction coefficients, the presence of baroclinic instability in the recirculation area prohibited the existence of multiple dynamical regimes. However, weak low frequency variability associated with changes in the structure and the 
(a)

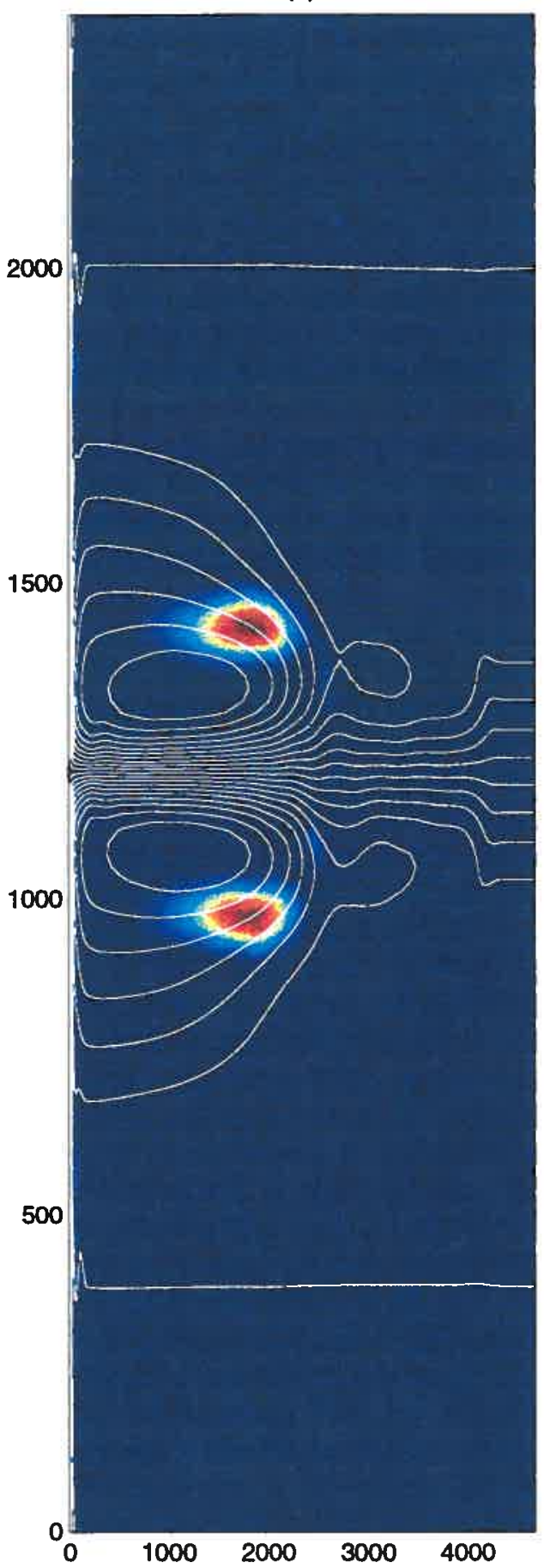

(b)

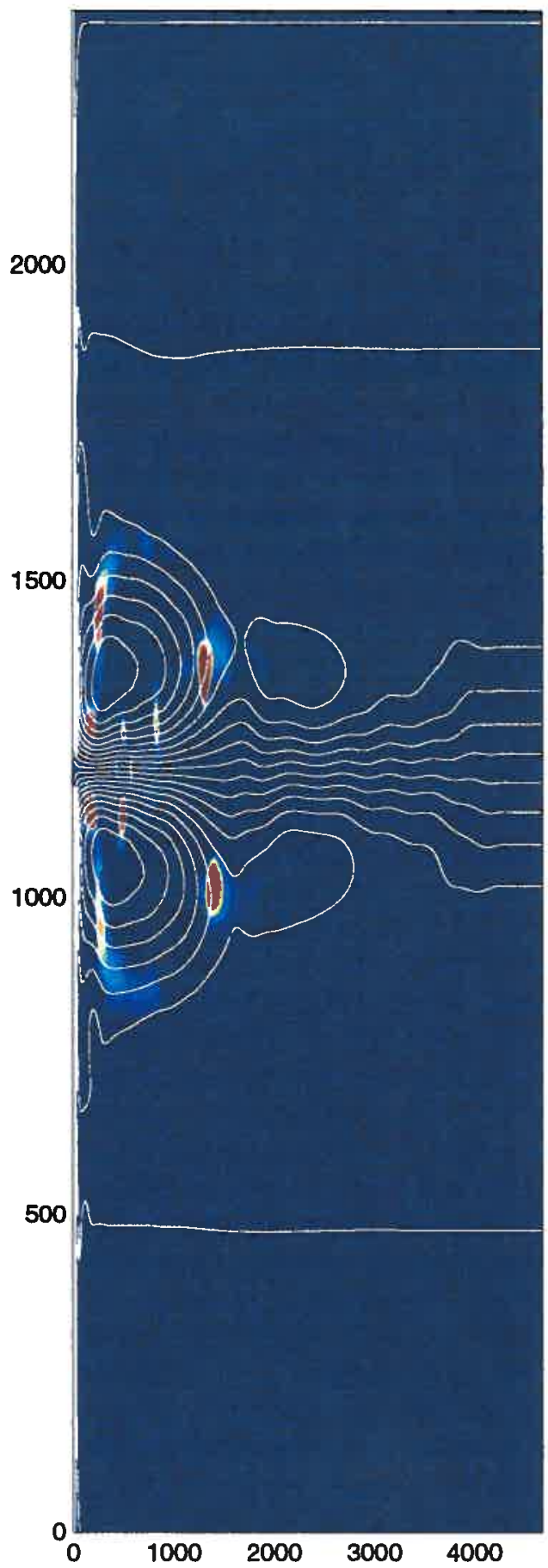

Figure 5-18: Zones of baroclinic growth for $A_{2}=10^{-8} \sec ^{-1}$ (a) and $A_{2}=3 \cdot 10^{-6}$ $\sec ^{-1}$ (b). White contours show upper layer time mean streamfunction field. 
strength of the recirculating gyres was still possible. In contrast with the reducedgravity case, where steady antisymmetric solutions helped to understand the existence and the structure of a high energy state and the associated dynamical regime, an attempt to find steady antisymmetric solutions by employing a two-layer model with enforced antisymmetry failed. In disagreement with the original time dependent model, where the zonal extent of the recirculations varied between $500 \mathrm{~km}$ and 2500 $\mathrm{km}$, the recirculations reached all the way to the eastern sponge layer, and the flow was characterized by a statistically steady nonzero antisymmetric eddy field. Therefore, the total energy level never reached a steady state. One of the reasons why the antisymmetric steady state did not exist may be that the upper layer dissipation mechanism was presented only by biharmonic diffusion, which is not efficient at dissipating energy. Therefore, the balance between the nonlinear terms and dissipation was not possible, and time variability was essential. The use of lateral diffusion might have resulted in existence of steady antisymmetric solutions. However, the question still remains whether the corresponding steady states would have been relevant to the behavior of the time-dependent model. As the system becomes progressively more complex, as in the case with both barotropic and baroclinic instability at work, the model might become purely turbulent, and the resulting dynamical state may be a strange attractor for a given parameter regime.

In contrast, in the two-layer, wind-driven, quasi-geostrophic model by Berloff and McWilliams [4], rare transitions between three distinct dynamical states were possible. Compared with a corresponding reduced-gravity, wind-driven model, the presence of baroclinic instability weakened and regularized low frequency variability. 


\section{Chapter 6}

\section{Conclusions}

This thesis studies the problems of generation and maintenance of recirculations by the Gulf Stream instabilities using two approaches. The first examines the development of recirculations during the spin down of a zonally symmetric, unstable, quasigeostrophic jet. The second studies the problem of existence and maintenance of low frequency variability and of multiple dynamical regimes due to the mechanisms of barotropic and baroclinic instabilities.

Chapter 2 addresses the first problem in a framework of the reduced-gravity and the two-layer doubly-periodic, quasi-geostrophic models. The recirculations are robust features of a two-layer model, which includes the mechanisms of both baroclinic and barotropic instabilities. The relative strength of the recirculations, defined as the ratio of the total westward to the total eastward transport, is a function of the nondimensional parameters of the model. It decreases as the value of nondimensional $\beta$ increases. This decrease in the recirculation strengths is due to a decrease in the zone of homogenized potential vorticity, which in turn is due to (approximate) enstrophy conservation.

When $\beta, \gamma$ and total energy level are fixed, the strength of the recirculations is maximum for strongly baroclinic flows and minimum for intermediate values of a baroclinic parameter, $\frac{u_{1}-u_{2}}{F_{1} u_{2}+F_{2} u_{1}}$, where $u_{i}$ is $i$-th layer nondimensional velocity amplitude 
and $F_{i}$ is $i$-th layer Froude number. In strongly baroclinic cases, eddies marginally equilibrate the flow and create a wide zone of homogenized potential vorticity in the lower layer. This result is in agreement with the study of a weakly-nonlinear equilibration of Phillips' model with weak dissipation (Pedlosky [28]). In the upper layer, a positive potential vorticity gradient on the axis of the jet decreases by less than $10 \%$, thus appearing as an efficient barrier to mixing. Most vigorous mixing occurs on the edges of the jet in the upper layer and under the axis of the jet in the lower layer.

As the initial basic jet becomes more barotropic, a positive potential vorticity gradient develops on the axis of the jet in the lower layer, reflecting a more stable flow structure. In strongly barotropic jets, there is vigorous mixing both across the positive potential vorticity gradient and on the edges of the jet, leading to a decrease by more than $45 \%$ in the value of the upper layer potential vorticity gradient on the axis of the jet.

The linear stability analysis applied to the two-layer jets shows that in strongly baroclinic cases, the lower layer recirculations are driven by the eddy heat fluxes through the residual meridional circulation. In the upper layer, the divergence of eddy potential vorticity fluxes tends to simply "diffuse" the jet during the stage of linear growth. Nonlinear eddy-eddy and eddy-mean flow interactions are further important in inducing the upper layer recirculations. The linear analysis shows that the strength of the residual meridional circulations increases with an increase in the width of basic flow. That is why a "mean flow" model (Flierl, personal communication) successfully reproduces recirculations when applied to strongly baroclinic basic flows.

For strongly barotropic two-layer basic flows, which are strongly supercritical, the linear eddy potential vorticity fluxes tend to diffuse the jet in both layers without any tendency for the formation of westward flows on the flanks. As the basic flow becomes wider, it stabilizes. Therefore, strongly nonlinear regimes, which allow for both eddy-mean flow and eddy-eddy interactions, are required for the emergence of recirculations for strongly barotropic basic flows. 
Thus, although the recirculations develop in a two-layer model for both barotropic and baroclinic flows, the processes leading to their generation are different. In baroclinic case, eddy-mean flow interaction is powerful enough to drive the recirculations. In barotropic cases, strongly nonlinear eddy-mean flow and eddy-eddy interactions are required for the development of recirculations. The existence of two different mechanisms is responsible for a non-monotonic dependence of the recirculation strength on the baroclinic parameter in the nonlinear experiments.

In the reduced-gravity model, which has the mechanism of barotropic instability only, recirculations form as a result of a nonlinear spin down of an unstable basic flow only for the case of an infinite dimensional deformation radius. For deformation radii smaller than $65 \mathrm{~km}$, the total westward transport is less than $5 \%$ of the total eastward transport.

The results of the linear stability analysis are misleading since they imply a weak tendency for the formation of the recirculations for small dimensional deformation radii and the absence of the recirculations for an infinite dimensional deformation radius. The linear stability analysis is not helpful since the basic flow is only slightly supercritical for large $\gamma$, implying a weak eddy field.

The second part of the thesis examines the problem of existence and maintenance of low frequency variability and of multiple dynamical regimes as the result of barotropic and baroclinic instabilities.

Chapter 3 presents the formulation of a quasi-geostrophic, colliding jets model which is forced by prescribed inflow and outflow through the open boundaries. The geometry and the amplitude of such boundary forcing is important for the resulting structure of a time-dependent solution. The inflow consists of two thin jets entering the domain from the north and the south and flowing along the closed western boundary. After colliding in the middle of the domain, the western boundary currents give rise to an eastward flowing jet that leaves the domain through the eastern boundary. A free slip boundary condition is applied on the western boundary, which 
tends to suppress local instabilities of the western boundary currents. The presence of the western wall and western boundary currents is crucial for the the existence of multiple dynamical regimes.

The model has two types of friction: biharmonic diffusion and interfacial drag in a reduced-gravity formulation and biharmonic diffusion and bottom drag in a twolayer formulation. In addition, the sponge layers located along the open boundaries allow the absorption of the outgoing radiation and provide a smooth transition between the interior solution and the boundary conditions.

Although negligible in the local potential vorticity balance of the recirculation regions, the wind is nevertheless important for the existence and maintenance of multiple dynamical regimes in the wind-driven models. First, the model energy input, defined as the domain-integrated streamfunction multiplied by the wind-stress curl, is time-dependent. When the nonlinear interactions are at work, the correlation between the spatial structures of the wind-stress curl and the solution can help the model trajectory to reach a linear neighborhood of an antisymmetric fixed point and therefore to lead the model towards a high energy state. Second, the potential vorticity input into various water masses, such as the recirculations, depends strongly on the structure of the solution. For nonsymmetric solutions, where one of the recirculations is wrapping around the other one and is crossing the line of zero wind-stress curl, the potential vorticity forcing integrated over a closed streamline decreases in absolute values compared to that of the antisymmetric solutions. Thus, in the wind-driven models, there are two possible sources of low frequency variability: wind forcing and instability mechanisms.

The colliding jets model is highly idealized, however, it isolates the important mechanisms of instability: barotropic in a reduced-gravity case and both barotropic and baroclinic in a two-layer case. The model differs from the wind-driven ones in that the energy input due to inflow through the open boundaries is time-independent. The boundary forcing does not act directly in the recirculation region and has no mecha- 
nisms that would locally restore the symmetry of the solution structure. Therefore, the observed high energy states are more transient than those in the wind-driven cases with symmetric wind.

Chapter 4 reports the results of the numerical experiments with the reducedgravity, colliding jets model. For a wide range of interfacial friction coefficients, steady antisymmetric solutions of the reduced-gravity, colliding jets model consist of an eastward jet flanked by two recirculating gyres. For a set of chosen parameters, the zonal extent of recirculations and the total energy level increase with a decrease in the interfacial friction coefficient.

The initial value technique was used to calculate the exponentially growing solutions of the antisymmetric states. The amplitude of the unstable mode closely resembles that of the unstable solution of the wind-driven reduced-gravity model of Primeau [29] and consists of four strong cells sitting on the axis of the jet between the recirculating gyres and weaker cells in the recirculation regions. The e-folding time varies between 4.8 years and 417 days. The linear growth in the perturbation amplitude halts when the nonlinear interactions of the cells become strong. These interactions lead to formation of rings in the area where the jet widens.

Fully nonlinear calculations show that with an increase in the value of the interfacial friction coefficient, the model becomes weakly unstable and displays a unique dynamical regime corresponding to an eastward jet with two recirculating gyres. For near critical values of the friction coefficient, the eddy field is dominated by eastward and westward propagating meanders of small amplitudes. Rings are weak and therefore dissipate during their propagation through the recirculation regions. The energy level and the flow structure are close to those of the steady antisymmetric solution.

For smaller values of the interfacial friction coefficient, the increased nonlinearity leads to low frequency variability associated with changes in the zonal extent of the recirculating gyres. Formation of strong rings in the area east of the recircula- 
tions and their subsequent westward propagation through the gyres provides intense potential vorticity mixing across the front and in the area of the recirculations. Rings are strong enough not to be completely dissipated in the recirculations; upon reaching a western boundary, they rejoin the eastward jet. Therefore, the model exhibits periods of large recirculations and weak eddy activity, when the model trajectory is in the vicinity of the antisymmetric fixed point, and periods of small recirculations and strong meander and ring activity.

With further decrease in the interfacial friction, the model displays two distinct energy states. The high energy state has strong variability associated with welldeveloped recirculations of various zonal extents, described in the above paragraph. The low energy state corresponds to a flow regime where the separation point of the eastward jet exhibits large meridional excursions. Weak recirculations exist west of $1000 \mathrm{~km}$ only in the time mean sense. The strong eddy field is dominated by large amplitude, westward propagating meanders whose interaction with the western boundary current produces strong meridionally propagating rings. In comparison, McCalpin and Haidvogel [25] identified three preferred dynamical regimes each with a distinct energy level. Although the high and the medium regimes resemble the variability associated with the high energy state of the regional model, the low energy state is quite different from the one of the regional model. This is partly because the outflow conditions of the colliding jets model force the eastward jet to stay coherent, and therefore the model never reaches the point when the jet disappears almost right after separation, as in a low energy state of the wind-driven model.

For low values of the interfacial friction coefficient, the flow stays in the dynamical regime described as a low energy state in the above paragraph. The amplitude of the eddy field is very strong, and small and weak recirculations exist only in the time mean sense.

In a regional colliding jets model, the role of wind forcing is reduced to prescribing a particular distribution of streamfunction and potential vorticity on the 
open boundaries. The local nature of such forcing allows isolating the role of internal barotropic dynamics of the jet and its interaction with the western boundary current as mechanisms responsible for the existence of low frequency variability and multiple dynamical regimes. The numerical experiments with the regional model show that low frequency variability is an inherent part of the barotropic dynamics where nonlinearities are not too weak or too strong. The presence of the western wall is essential, since no low frequency variability or multiple dynamical regimes were observed in a barotropic, zonally asymmetric model of Jayne et al. [18], which was forced by an unstable zonal jet on the open western boundary and a stable zonal jet on the open eastern boundary.

One of the most important properties of the regional model is a constant energy input, given by the boundary integrated energy flux vector. In comparison, in the wind-driven models, the forcing has global character, and the energy input strongly depends on the solution form. Scott and Straub [34] discussed a correlation mechanism between symmetric wind-forcing and a geostrophic current which led to ability of steady antisymmetric solutions to extract more energy than nonsymmetric solutions. The experiments with various wind asymmetry parameter in the model of McCalpin and Haidvogel [25] showed that for highly asymmetric wind forcing, the model was not able to reach high energy states. Since the regional model does not have any mechanism that restores the symmetry of the solution, it is harder for the model trajectory to reach and to stay in the vicinity of the antisymmetric steady solution.

In the present study, we use a free slip boundary condition on the western boundary. As Berloff and McWilliams [3] showed, the presence of a no-slip boundary condition tends to destabilize western boundary currents. Eddies produced by such currents can be advected towards the middle of the domain and may interact with rings that propagate towards the western wall from the model's interior. Hence, the nature of low frequency variability and of multiple dynamical regimes might change 
with the use of no-slip boundary condition due to more complicated dynamics of the western boundary current. This problem, however, is not addressed in the present thesis.

In Chapter 5, we describe the results of the numerical experiments with a twolayer, colliding jets model which shows strong dependence of the flow structure on the bottom friction parameter. For small bottom friction, which implies a physically relevant lower layer flow, the model stays in a unique dynamical regime associated with the well-developed recirculations in both layers. To understand the nature of low and high frequency variability observed in the model, an extended spatial EOF analysis was performed for filtered streamfunction fields. The low frequency spatial EOFs are found to explain decadal and longer variability in the position of the eastward jet axis, as well as weak wrapping and zonal pulsations of the recirculation gyres. For quasi-annual and mesoscale variability, the EOF analysis shows the importance of wave-like structures sitting on the axis of the jet between the recirculating gyres with the length scale of $1000 \mathrm{~km}$ and the time scale of 8 to 9 months. The results of the EOF analysis for the regional model differ from the stability calculations of the zonally-symmetric jet, where the time scales are much shorter: typically, between 2 and 4 months.

For large values of the bottom friction coefficient, the lower layer flow becomes strongly frictional, and the recirculations disappear. However, the dynamics of the upper layer flow becomes more complicated displaying both multiple dynamical regimes and low frequency variability which closely resemble those of the reduced-gravity model with weak interfacial friction. Such similarity between the reduced-gravity and the two-layer models is possible due to forcing provided by the lower layer flow on the upper layer flow, associated with strong bottom friction.

The results of the two-layer calculations show that for physically feasible values of the bottom friction coefficients, the presence of baroclinic instability in the recirculation area prohibits the existence of multiple dynamical regimes. However, weak 
low frequency variability associated with changes in the structure and the strength of the recirculating gyres is still possible. In contrast with the reduced-gravity case, where steady antisymmetric solutions are useful for understanding the existence and the structure of a high energy state and the associated dynamical regime, an attempt to find steady antisymmetric solutions by employing a two-layer model with enforced antisymmetry failed. In disagreement with the original time dependent model, where the zonal extent of the recirculations varies between $500 \mathrm{~km}$ and $2500 \mathrm{~km}$, the recirculations reach all the way to the eastern sponge layer, and the flow is characterized by a statistically steady nonzero antisymmetric eddy field. Therefore, the total energy level never reaches a steady state. One of the reasons why the antisymmetric steady state does not exist may be that the upper layer dissipation mechanism is presented only by biharmonic diffusion, which is not efficient at dissipating energy. Therefore, the balance between the nonlinear terms and dissipation is not possible, and time variability is essential. The question still remains whether the corresponding steady states are relevant to the behavior of the time-dependent model. As the system becomes progressively more complex, as in the case with both barotropic and baroclinic instability at work, the model might become purely turbulent, and the resulting dynamical state may be a strange attractor for a given parameter regime.

In contrast, in the two-layer, wind-driven, quasi-geostrophic model by Berloff and McWilliams [4], rare transitions between three distinct dynamical states were possible. Compared with a corresponding reduced-gravity, wind-driven model, the presence of baroclinic instability weakened and regularized low frequency variability. When interpreting the results of the reduced-gravity and the two-layer colliding jets models, one must remember that they are highly idealized. One of the basic simplifications used in the present study is the quasi-geostrophic nature of the flow, which overemphasizes the importance of horizontal structure of the ocean and which allows for only small perturbation of pressure and density surfaces from their original position. And although the quasi-geostrophic approximation proved to be a 
useful tool in studying the mid-latitude ocean dynamics, a primitive equation model might halt low frequency variability. Even within the quasi-geostrophic approximation, consideration of continuously stratified fluid might further reduce low frequency variability.

Low-frequency variability is well documented in observations of the world oceans. Twenty two years of hydrographic data along $137^{\circ} \mathrm{E}$ show strong inter-annual bimodal path variations in the Kuroshio ( Qiu and Joyce [30]). The net transport of the Kuroshio system which includes the jet and its recirculations increases by $30 \%$ during the meander-path years. The increase is primarily due to the decrease in the recirculation strength. Thus, recirculations are strongest during the straight-path years. Observations of East Australian current (Roemmich and Cornuelle [33]) and of Brazil and Malvinas currents (Olson et al. [26]) also display strong inter-annual variability. Observations of sea surface height in the Gulf Stream area by Kelly et al. [19] show a trend over two years from a state with a weakly-meandering jet and strong recirculations to a state with a strongly meandering jet and small recirculations. Sea surface temperature observations by Lee and Cornillon [21] indicate annual and inter-annual oscillations in the position of the Gulf Stream path.

Although the colliding jets model isolating the internal dynamics of the jet as sources of low frequency variability is highly idealized, it produces similar variability for a wide range of dissipation parameters: namely, pulsations in the zonal extent of the recirculations, weak wrapping of the recirculations around each other and meridional shifts in the position of the jet. Another interesting feature of the model is the importance of wave-like structures with time scales between 9 months and 1.3 years. Lee and Cornillon [21] indicated the importance of a 9-month cycle in the meandering intensity of the jet. Clearly, the zonally symmetric model was not able to reproduce this feature, since most energetic wave had much shorter time scales.

Perhaps, in the real ocean, there are several mechanisms that could be responsible for structural changes in the jet and in the recirculation area, such as the 
presence of time varying winds, complicated topography, the instabilities of the jet and the inherent dynamics of the steady wind-driven circulation. The colliding jets model isolates the importance of one of them, the internal dynamics of the jet, and shows the differences between baroclinic and barotropic instabilities as sources of low frequency variability. Further studies are necessary for understanding of the role of time-varying winds and topography in low-frequency variability. 


\section{Bibliography}

[1] Andrews, D. G. and McIntire, M. E., 1976: Planetary waves in horizontal and vertical shear, the generalized Ellissen-Palm relation and the mean zonal acceleration. J. Atmos. Sci., 33, 2031-2048.

[2] Arnold, V. I., 1965: Conditions for nonlinear stability of stationary plane curvilinear flows of an ideal fluid. Dokl. Akad. Nauk SSSR, 162, 975-978.

[3] Berloff, P. S. and J.C. McWilliams, 1998: Quasi-geostrophic dynamics of the Western Boundary Current. submitted

[4] Berloff, P. S. and J.C. McWilliams, 1998: Large-Scale, Low Frequency Variability in Wind-Driven Ocean Gyres. submitted

[5] Cessi, P., G. R. Ierley and W. R. Young, 1987: A model of the inertial recirculation driven by potential vorticity anomalies. J. Phys. Oceanogr., 17, 1640-1652.

[6] Cessi, P., 1988: A stratified model of the inertial recirculation. J. Phys. Oceanogr., 18, 662-682.

[7] Davis C. A. and K. A. Emanuel, 1991: Potential vorticity diagnostics of cyclogenesis. Mon. Weather. Rev., 119(8), 1929-1953.

[8] Fofonoff, N. P., 1962: Dynamics of ocean currents. The Sea, Vol. 1: Physical Oceanography., M. N. Hill, Ed., Wiley-Interscience. 
[9] Greatbatch, R. J., 1987: A model for the inertial recirculation of a gyre. J. Mat. Res., 45, 601-634.

[10] Hogg, N. G., 1983: A note on the deep circulation of the western North Atlantic: its nature and causes. Deep Sea Res., 30, 945-961.

[11] Hogg, N. G., 1988: Stochastic wave radiation by the Gulf Stream, J. Phys. Oceanogr., 18, 1687-1701.

[12] Hogg, N. G., 1992: On the transport of the Gulf Stream between Cape Hatteras and the Grand Banks. Deep Sea Res., 39, 1231-1246.

[13] Hogg, N. G., 1993: Toward parameterization of the eddy field near the Gulf Stream. Deep Sea Res., 40, 2359-2376.

[14] Hogg, N. G., R. S. Pickart, R. M. Hendry and W. Smethie, 1986: The Northern Recirculation Gyre of the Gulf Stream. Deep Sea Res., 33(9A), 1139-1165.

[15] Holland, W. R., 1978: The Role of Mesoscale Eddies in the General Circulation of the Ocean - Numerical Experiments Using a Wind-Driven Quasi-Geostrophic Model. J. Phys. Oceanogr., 8, 363-392.

[16] Holland, W. R. and P. B. Rhines, 1980: An example of eddy-induced ocean circulation. J. Phys. Oceanogr., 10, 1010-1031.

[17] Holland, W. R., D. E. Harrison and A. J. Semtner, 1983: Eddy-resolving numerical models of large-scale ocean circulation. In Eddies in Marine Science, ed. A.R. Robinson, 379-403.

[18] Jayne, S. R., Hogg, N. G., Malanotte-Rizzoli, P., 1996: Recirculation gyres forced by a beta-plane jet. J. Phys. Ocean., 26, 492-504. 
[19] Kelly, K. A., Caruso, M. J., Singh, S., and Bo Qui, 1996: Observations of atmosphere-ocean coupling in midlatitude western boundary currents. J. Geophys. Res., 101, 6295-6312.

[20] Lee T., Cornillon P., 1996: Propagation and growth of Gulf Stream meanders between 75 degrees and 45 degrees W. J. Phys. Ocean., 26, 225-241.

[21] Lee T., Cornillon P., 1996: Temporal variation of meandering intensity and domain-wide lateral oscillations of the Gulf Stream. J. Geophys. Res., 100, 13603-13613.

[22] Malanotte-Rizzoli, P., D. B. Haidvogel and R. E. Young, 1987: Numerical simulation of transient boundary-forced radiation. Part I: the linear regime. J. Phys. Oceanogr., 17, 1439-1457.

[23] Malanotte-Rizzoli, P., N. G. Hogg and R. E. Young, 1994: Stochastic wave radiation by the Gulf Stream: numerical experiments. Deep Sea Res., 42, 389423.

[24] Marshall, J. C. and G. Nurser, 1986: Steady, free circulation in a quasigeostrophic ocean. J. Phys. Oceanogr., 16, 1799-1813.

[25] McCalpin, J. D., and D. B. Haidvogel, 1996: Phenomenology of the low frequency variability in a reduced-gravity, quasi-geostrophic double-gyre model. J. Phys. Oceanogr., 26, 739-752.

[26] Olson, D. B., G. P. Podesta, R. H. Evans and O. B. Brown, 1988: Temporal variations in the separation of Brazil and Malvinas Currents. Deep-Sea Res., 35, 1971-1990.

[27] Pedlosky, J., 1987. Geophysical Fluid Dynamics, 2nd edn. Springer-Verlag, 710 pp. 
[28] Pedlosky, J., 1982: A simple model for nonlinear critical layers in an unstable baroclinic wave. J. Atmos. Sci.., 39, 2119-2127.

[29] Primeau, F., 1998. Multiple equilibria and low frequency variability of wind-driven ocean models, MIT Ph.D. Thesis, 158 pp.

[30] Qui, B. and T. Joyce, 1992: Inter-annual variability in the mid- and low-latitude western North Pacific. J. Phys. Ocean., 22, 1062-1079.

[31] Richardson, P. L., 1980: Gulf Stream ring trajectories. J. Phys. Oceanogr.., 10, 90-104.

[32] Richardson, P. L., 1985: Average velocity and transport of the Gulf Stream near 55W. J. Mar. Res., 43, 83-111.

[33] Roemmich, D. and B. Cornuelle, 1990: Observing fluctuations of gyre scale ocean circulations: a study of the sub-tropical South Pacific. J. Phys. Ocean., 20, 1919-1934.

[34] Scott, R. B., and D. N. Straub, 1997: Small viscosity behavior of a homogeneous, quasi-geostrophic ocean circulation Model. in press

[35] Shepherd, T. D., 1983: Mean motions induced by baroclinic instability in a jet. Geophys. Astrophys. Fluid Dynamics, 27, 35-72.

[36] Simmons, A. J. and B. J. Hoskins, 1976: Baroclinic instability on the sphere: normal modes of the primitive and quasi-geostrophic equations. J. Atmos. Sci., $33,1454-1477$.

[37] Schmitz, W. J. Jr., 1980: Weakly depth-dependent segments on the North Atlantic circulation. J. Mar. Res., 38, 111-133. 
[38] Spall, M. A., 1996: Dynamics of the Gulf Stream/Deep Western Boundary current crossover. Part I: Entrainment and recirculations. J. Phys. Oceanogr., 26, $2152-2168$.

[39] Spall, M. A., 1996: Dynamics of the Gulf Stream/Deep Western Boundary current crossover. Part II: low frequency internal recirculations. J. Phys. Oceanogr., 26, 2169-2182.

[40] Worthington, L. V., On the North Atlantic Circulation, The Johns Hopkins Oceanographic Studies, 6, Johns Hopkins Univ Press, Baltimore, 110pp.

[41] Wunsch, C., 1978: The general circulation of the North Atlantic west of $50^{\circ} \mathrm{W}$ determined from inverse methods. Reviews of Geophysics and Space Physics, 16, $583-620$.

[42] Wunsch, C. and B. Grant, 1982: Towards the general circulation of the North Atlantic Ocean. Progress in Oceanography, 11, 1-59. 